Supporting Information for:

\title{
2,3-Anhydrosugars in Glycoside Bond Synthesis. Application to $\alpha$-D-Galactofuranosides
}

\author{
Yu Bai and Todd L. Lowary*,\# \\ Alberta Ingenuity Centre for Carbohydrate Science and Department of Chemistry, The \\ University of Alberta, Gunning-Lemieux Chemistry Centre, \\ Edmonton, AB T6G 2G2, Canada. \\ Email: tlowary@ualberta.ca
}

\section{Table of Contents}

Synthesis of 34-37 and measurement of ${ }^{1} J_{\mathrm{C} 1, \mathrm{H} 1}$ values.

Additional experimental details and data for new compounds

S8-S32

References for Supporting Information

S32

${ }^{1} \mathrm{H}$ NMR spectrum of $\mathbf{1 3}$

S33

${ }^{13} \mathrm{C}$ NMR spectrum of $\mathbf{1 3}$

S34

${ }^{1} \mathrm{H}$ NMR spectrum of $\mathbf{1 4}$

S35

${ }^{13} \mathrm{C}$ NMR spectrum (APT) of $\mathbf{1 4}$

S36

${ }^{1} \mathrm{H}$ NMR spectrum of $\mathbf{1 5}$

S37

${ }^{13} \mathrm{C}$ NMR spectrum (APT) of $\mathbf{1 5}$

S38

${ }^{1} \mathrm{H}$ NMR spectrum of $\mathbf{1 6 a}$

S39

${ }^{13} \mathrm{C}$ NMR spectrum (APT) of $\mathbf{1 6 a}$

S40

${ }^{1} \mathrm{H}$ NMR spectrum of $\mathbf{1 6 b}$ 
${ }^{13} \mathrm{C}$ NMR spectrum (APT) of $\mathbf{1 6 b} \quad$ S42

${ }^{1} \mathrm{H}$ NMR spectrum of $\mathbf{1 7 a} \quad$ S43

${ }^{13} \mathrm{C}$ NMR spectrum (APT) of $\mathbf{1 7 a} \quad \mathrm{S} 44$

${ }^{1} \mathrm{H}$ NMR spectrum of $\mathbf{1 7 b} \quad \mathrm{S} 45$

${ }^{13} \mathrm{C}$ NMR spectrum (APT) of $\mathbf{1 7 b} \quad$ S46

${ }^{1} \mathrm{H}$ NMR spectrum of $\mathbf{1 8 a} \quad$ S47

${ }^{13} \mathrm{C}$ NMR spectrum (APT) of 18a $\quad$ S48

${ }^{1} \mathrm{H}$ NMR spectrum of $\mathbf{1 8 b} \quad$ S49

${ }^{13} \mathrm{C}$ NMR spectrum (APT) of $\mathbf{1 8 b} \quad$ S50

${ }^{1} \mathrm{H}$ NMR spectrum of 21

${ }^{13} \mathrm{C}$ NMR spectrum (APT) of 21

${ }^{1} \mathrm{H}$ NMR spectrum of 22

${ }^{13} \mathrm{C}$ NMR spectrum (APT) of 22

${ }^{1} \mathrm{H}$ NMR spectrum of $\mathbf{2 3} \quad$ S55

${ }^{13} \mathrm{C}$ NMR spectrum (APT) of $\mathbf{2 3} \quad$ S56

${ }^{1} \mathrm{H}$ NMR spectrum of $\mathbf{2 6} \quad$ S57

${ }^{13} \mathrm{C}$ NMR spectrum (APT) of $\mathbf{2 6} \quad$ S58

$\begin{array}{ll}{ }^{1} \mathrm{H} \text { NMR spectrum of } \mathbf{3 3} & \text { S59 }\end{array}$

${ }^{13} \mathrm{C}$ NMR spectrum (APT) of $\mathbf{3 3} \quad$ S60

${ }^{1} \mathrm{H}$ NMR spectrum of $\mathbf{3 4} \quad$ S61

${ }^{13} \mathrm{C}$ NMR spectrum (APT) of $\mathbf{3 4} \quad$ S62

${ }^{1} \mathrm{H}$ NMR spectrum of $\mathbf{3 5} \quad$ S63

${ }^{13} \mathrm{C}$ NMR spectrum (APT) of $\mathbf{3 5} \quad$ S64

${ }^{1} \mathrm{H}$ NMR spectrum of $\mathbf{3 6} \quad$ S65

${ }^{13} \mathrm{C}$ NMR spectrum (APT) of $\mathbf{3 6} \quad$ S66

${ }^{1} \mathrm{H}$ NMR spectrum of $\mathbf{3 7} \quad$ S67

${ }^{13} \mathrm{C}$ NMR spectrum (APT) of $\mathbf{3 7} \quad$ S68 
$\begin{array}{ll}{ }^{1} \mathrm{H} \text { NMR spectrum of } \mathbf{4 6} & \text { S69 }\end{array}$

$\begin{array}{ll}{ }^{13} \mathrm{C} \text { NMR spectrum (APT) of } \mathbf{4 6} & \text { S70 }\end{array}$

$\begin{array}{ll}{ }^{1} \mathrm{H} \text { NMR spectrum of } \mathbf{4 7} & \text { S71 }\end{array}$

${ }^{13} \mathrm{C}$ NMR spectrum (APT) of $\mathbf{4 7} \quad \mathrm{S} 72$

$\begin{array}{ll}{ }^{1} \mathrm{H} \text { NMR spectrum of } \mathbf{4 8} & \text { S73 }\end{array}$

${ }^{13} \mathrm{C}$ NMR spectrum (APT) of $\mathbf{4 8} \quad$ S74

$\begin{array}{ll}{ }^{1} \mathrm{H} \text { NMR spectrum of } 49 & \text { S75 }\end{array}$

${ }^{13} \mathrm{C}$ NMR spectrum (APT) of $49 \quad$ S76

$\begin{array}{ll}{ }^{1} \mathrm{H} \text { NMR spectrum of } \mathbf{5 0} & \text { S77 }\end{array}$

${ }^{13} \mathrm{C}$ NMR spectrum (APT) of $\mathbf{5 0} \quad$ S78

$\begin{array}{ll}{ }^{1} \mathrm{H} \text { NMR spectrum of } \mathbf{5 1} & \text { S79 }\end{array}$

${ }^{13} \mathrm{C}$ NMR spectrum (APT) of $\mathbf{5 1} \quad$ S80

${ }^{1} \mathrm{H}$ NMR spectrum of $\mathbf{5 2} \quad$ S81

${ }^{13} \mathrm{C}$ NMR spectrum (APT) of $\mathbf{5 2} \quad$ S82

${ }^{1} \mathrm{H}$ NMR spectrum of $\mathbf{5 3} \quad$ S83

${ }^{13} \mathrm{C}$ NMR spectrum (APT) of $\mathbf{5 3} \quad$ S84

${ }^{1} \mathrm{H}$ NMR spectrum of $\mathbf{5 4} \quad$ S85

${ }^{13} \mathrm{C}$ NMR spectrum (APT) of $\mathbf{5 4} \quad$ S86

${ }^{1} \mathrm{H}$ NMR spectrum of $\mathbf{5 5} \quad$ S87

${ }^{13} \mathrm{C}$ NMR spectrum (APT) of $\mathbf{5 5} \quad$ S88

$\begin{array}{ll}{ }^{1} \mathrm{H} \text { NMR spectrum of } \mathbf{5 6} & \text { S89 }\end{array}$

${ }^{13} \mathrm{C}$ NMR spectrum (APT) of $\mathbf{5 6} \quad$ S90

${ }^{1} \mathrm{H}$ NMR spectrum of $\mathbf{5 7} \quad$ S91

${ }^{13} \mathrm{C}$ NMR spectrum (APT) of $\mathbf{5 7} \quad$ S92

${ }^{1} \mathrm{H}$ NMR spectrum of $\mathbf{5 8} \quad$ S93

${ }^{13} \mathrm{C}$ NMR spectrum (APT) of $\mathbf{5 8} \quad$ S94

${ }^{1}$ H NMR spectrum of $\mathbf{5 9} \quad$ S95 
${ }^{13} \mathrm{C}$ NMR spectrum (APT) of $\mathbf{5 9} \quad$ S96

${ }^{1} \mathrm{H}$ NMR spectrum of $\mathbf{6 0} \quad$ S97

${ }^{13} \mathrm{C}$ NMR spectrum (APT) of $60 \quad$ S98

${ }^{1} \mathrm{H}$ NMR spectrum of $\mathbf{6 1} \quad$ S99

${ }^{13} \mathrm{C}$ NMR spectrum (APT) of $\mathbf{6 1} \quad \mathrm{S} 100$

${ }^{1} \mathrm{H}$ NMR spectrum of $\mathbf{6 4} \quad$ S101

${ }^{13} \mathrm{C}$ NMR spectrum (APT) of $\mathbf{6 4} \quad$ S102

${ }^{1} \mathrm{H}$ NMR spectrum of $\mathbf{6 5} \quad$ S103

${ }^{13} \mathrm{C}$ NMR spectrum (APT) of $\mathbf{6 5} \quad \mathrm{S} 104$

${ }^{1} \mathrm{H}$ NMR spectrum of $\mathbf{6 6 / 6 7} \quad$ S105

${ }^{13} \mathrm{C}$ NMR spectrum (APT) of $\mathbf{6 6 / 6 7} \quad$ S106

$\begin{array}{ll}{ }^{1} \mathrm{H} \text { NMR spectrum of } \mathbf{6 8} & \text { S107 }\end{array}$

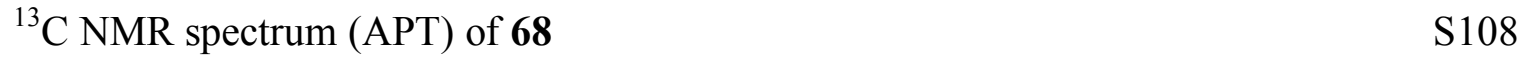

${ }^{1} \mathrm{H}$ NMR spectrum of $69 \quad$ S109

${ }^{13} \mathrm{C}$ NMR spectrum (APT) of $69-\mathrm{S} 110$

${ }^{1} \mathrm{H}$ NMR spectrum of $\mathbf{7 2} \quad$ S111

${ }^{13}$ C NMR spectrum (APT) of $72 \quad$ S112

$\begin{array}{ll}{ }^{1} \mathrm{H} \text { NMR spectrum of } \mathbf{7 3} & \mathrm{S} 113\end{array}$

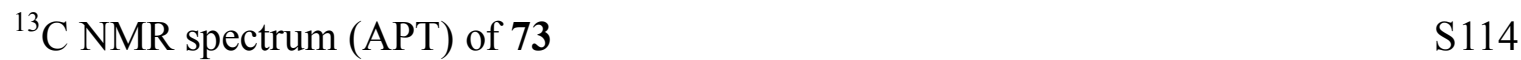

$\begin{array}{ll}{ }^{1} \mathrm{H} \text { NMR spectrum of } \mathbf{7 4} & \text { S115 }\end{array}$

${ }^{13} \mathrm{C}$ NMR spectrum (APT) of $\mathbf{7 4} \quad$ S116

$\begin{array}{ll}{ }^{1} \mathrm{H} \text { NMR spectrum of } \mathbf{7 6} & \text { S117 }\end{array}$

${ }^{13} \mathrm{C}$ NMR spectrum (APT) of $76 \quad$ S118

$\begin{array}{ll}{ }^{1} \mathrm{H} \text { NMR spectrum of } 77 & \text { S119 }\end{array}$

${ }^{13} \mathrm{C}$ NMR spectrum (APT) of $77 \quad$ S120

$\begin{array}{ll}{ }^{1} \mathrm{H} \text { NMR spectrum of } \mathbf{7 8} & \text { S121 }\end{array}$

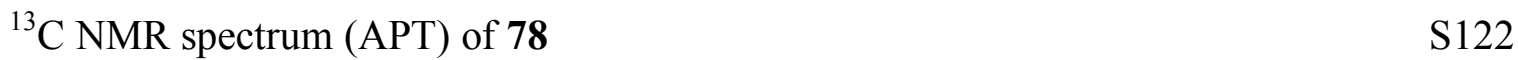


${ }^{1} \mathrm{H}$ NMR spectrum of $79 \quad$ S123

$\begin{array}{lr}{ }^{13} \mathrm{C} \text { NMR spectrum (APT) of } 79 & \text { S124 }\end{array}$

$\begin{array}{ll}{ }^{1} \mathrm{H} \text { NMR spectrum of } \mathbf{8 0} & \text { S125 }\end{array}$

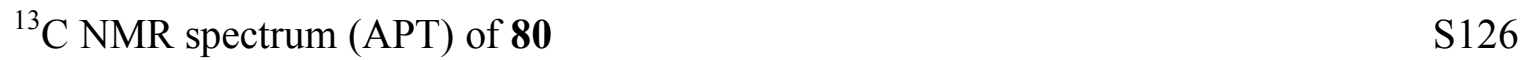

$\begin{array}{ll}{ }^{1} \mathrm{H} \text { NMR spectrum of } \mathbf{8 1} & \text { S127 }\end{array}$

${ }^{13} \mathrm{C}$ NMR spectrum (APT) of $\mathbf{8 1} \quad$ S128

$\begin{array}{ll}{ }^{1} \mathrm{H} \text { NMR spectrum of } \mathbf{8 2} & \text { S129 }\end{array}$

${ }^{13} \mathrm{C}$ NMR spectrum (APT) of $\mathbf{8 2} \quad$ S130

${ }^{1} \mathrm{H}$ NMR spectrum of $\mathbf{8 3} \quad$ S131

${ }^{13} \mathrm{C}$ NMR spectrum (APT) of $\mathbf{8 3} \quad$ S132

$\begin{array}{ll}{ }^{1} \mathrm{H} \text { NMR spectrum of } \mathbf{S 2} & \text { S133 }\end{array}$

${ }^{13} \mathrm{C}$ NMR spectrum (APT) of $\mathbf{S 2} \quad \mathrm{S} 134$

$\begin{array}{ll}{ }^{1} \mathrm{H} \text { NMR spectrum of } \mathbf{S 3} & \mathrm{S} 135\end{array}$

${ }^{13} \mathrm{C}$ NMR spectrum (APT) of $\mathbf{S 3} \quad$ S136

$\begin{array}{ll}{ }^{1} \mathrm{H} \text { NMR spectrum of } \mathbf{S 4} & \text { S137 }\end{array}$

${ }^{13} \mathrm{C}$ NMR spectrum (APT) of $\mathbf{S 4} \quad \mathrm{S} 138$

$\begin{array}{ll}{ }^{1} \mathrm{H} \text { NMR spectrum of S5 } & \text { S139 }\end{array}$

${ }^{13}$ C NMR spectrum (APT) of S5 $\quad$ S140

${ }^{1} \mathrm{H}$ NMR spectrum of $\mathbf{S 6} \quad$ S141

${ }^{13} \mathrm{C}$ NMR spectrum (APT) of S6 $\quad$ S142

$\begin{array}{ll}{ }^{1} \mathrm{H} \text { NMR spectrum of } \mathbf{S 7} & \text { S143 }\end{array}$

${ }^{13} \mathrm{C}$ NMR spectrum (APT) of S7 $\quad$ S144

$\begin{array}{ll}{ }^{1} \mathrm{H} \text { NMR spectrum of } \mathbf{S 8} & \text { S145 }\end{array}$

${ }^{13} \mathrm{C}$ NMR spectrum (APT) of $\mathbf{S 8} \quad$ S146 


\section{Synthesis of 34-37 and measurement of ${ }^{1} J_{\mathrm{C} 1, \mathrm{H} 1}$ values.}

The routes used for the synthesis of 34-37 are shown in Scheme S1 and S2. Starting from 22 (see main text of paper) and the known intermediates $\mathbf{4 1}$ and $\mathbf{S 1},{ }^{2}$ all four compounds could be obtained without difficulty as illustrated below. Once synthesized, the ${ }^{1} J_{\mathrm{C} 1 \text {,H1 }}$ values were extracted from ${ }^{1} \mathrm{H}$-coupled HMQC spectra.
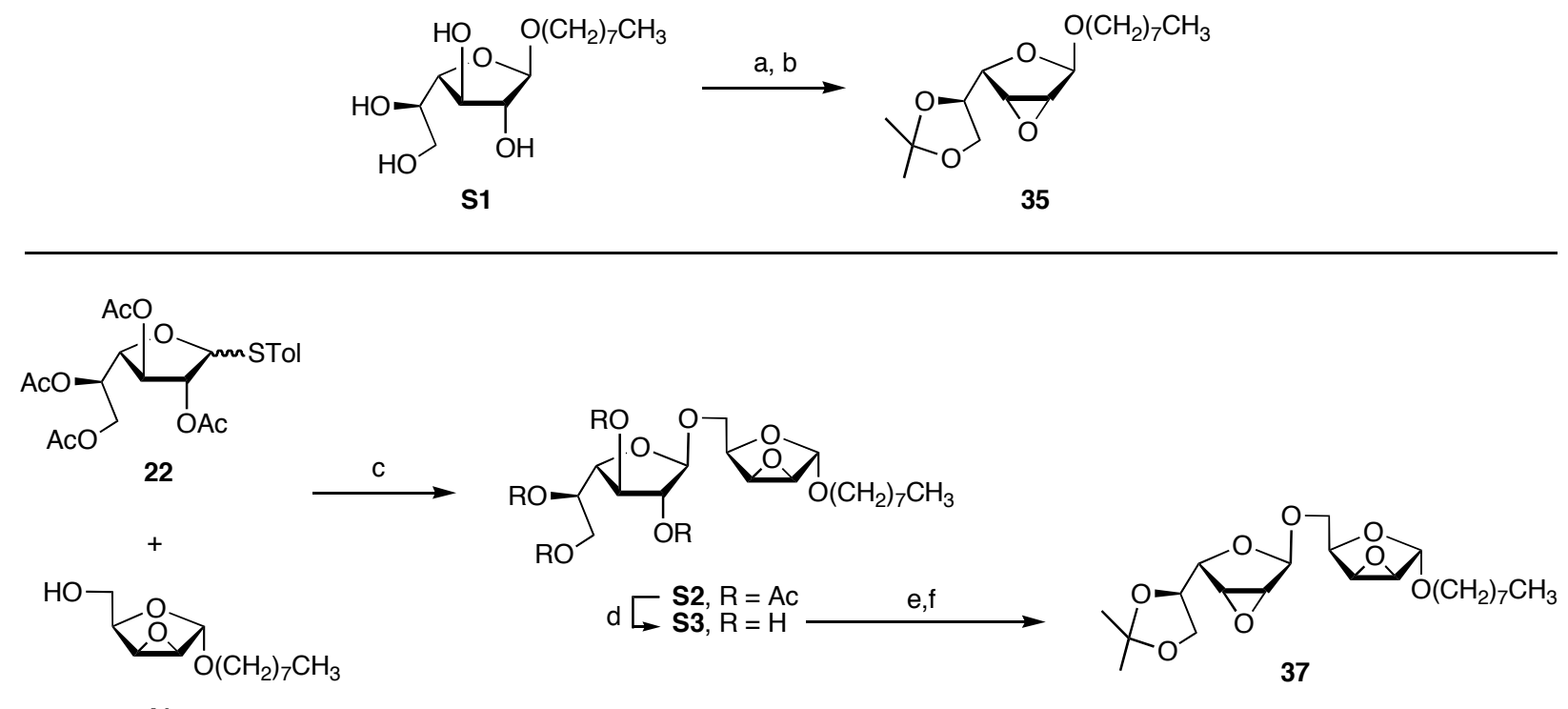

41

Scheme S1. (a) $\left(\mathrm{CH}_{3} \mathrm{O}\right)_{2} \mathrm{C}\left(\mathrm{CH}_{3}\right)_{2}$, acetone, $p$-TsOH, rt; (b) DIAD, $\mathrm{Ph}_{3} \mathrm{P}, \mathrm{THF}, 0{ }^{\circ} \mathrm{C} \rightarrow \mathrm{rt}$, 2 steps, 49\%; (c) NIS, AgOTf, $\mathrm{CH}_{2} \mathrm{Cl}_{2}, 0{ }^{\circ} \mathrm{C}, 89 \%$; (d) $\mathrm{NaOCH}_{3}, \mathrm{CH}_{3} \mathrm{OH}, \mathrm{rt}, 87 \%$; $\left(\mathrm{CH}_{3} \mathrm{O}\right)_{2} \mathrm{C}\left(\mathrm{CH}_{3}\right)_{2}$, acetone, $p$-TsOH, rt; (b) DIAD, $\mathrm{Ph}_{3} \mathrm{P}, \mathrm{THF}, 0^{\circ} \mathrm{C} \rightarrow \mathrm{rt}, 2$ steps, $74 \%$ 

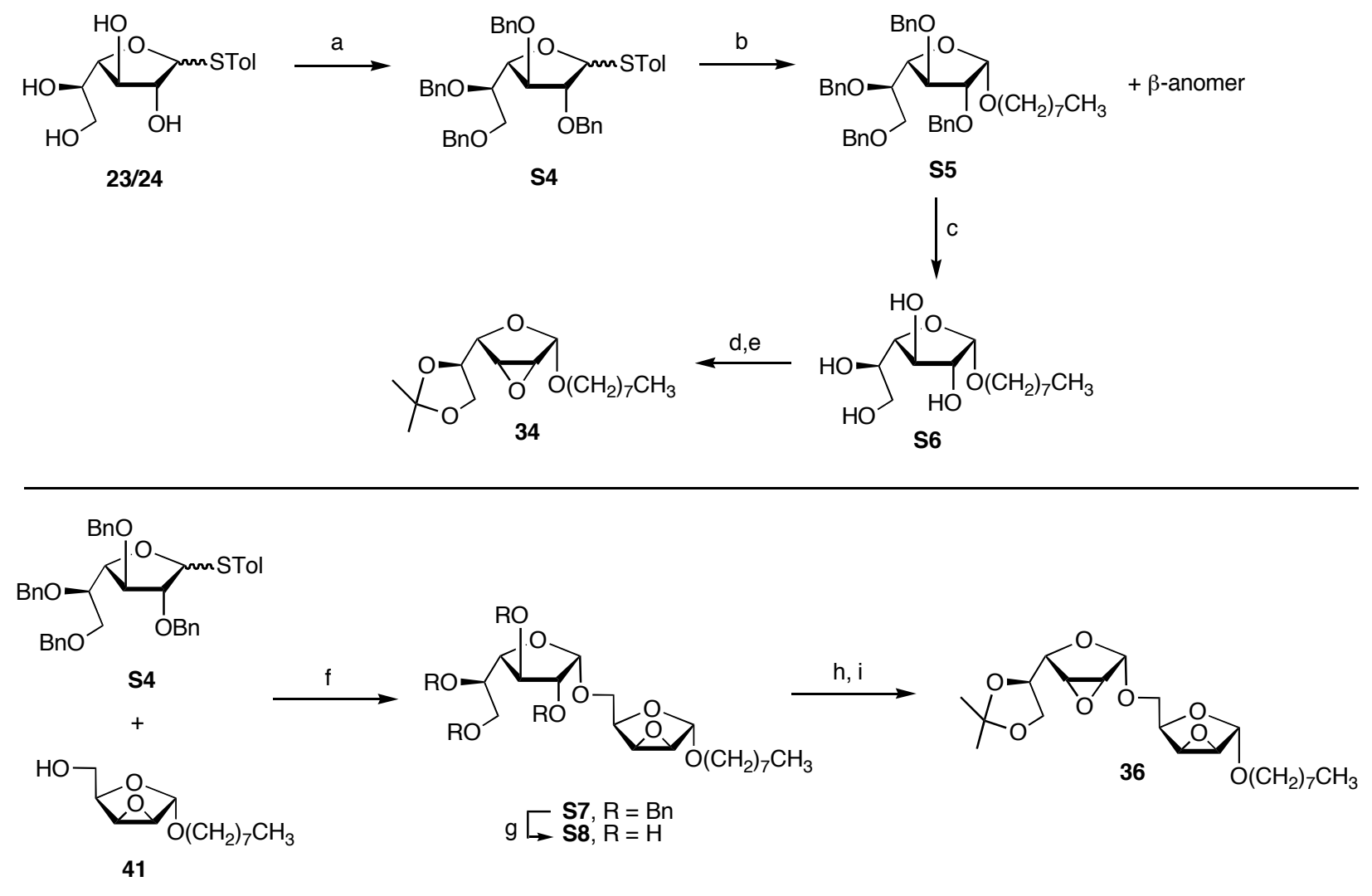

Scheme S2. (a) BnBr, NaH, DMF, $0{ }^{\circ} \mathrm{C} \rightarrow$ rt, 98\%; (b) $n$-octanol, NIS, AgOTf, $\mathrm{CH}_{2} \mathrm{Cl}_{2}, 0$ ${ }^{\circ} \mathrm{C}, 62 \%+17 \% \beta$-anomer; (c) $\mathrm{H}_{2}, \mathrm{Pd}(\mathrm{OH})_{2} / \mathrm{C}, \mathrm{CH}_{3} \mathrm{OH}, \mathrm{rt}, 85 \%$; (d) $\left(\mathrm{CH}_{3} \mathrm{O}\right)_{2} \mathrm{C}\left(\mathrm{CH}_{3}\right)_{2}$, acetone, $p$-TsOH, rt; (b) DIAD, $\mathrm{Ph}_{3} \mathrm{P}, \mathrm{THF}, 0{ }^{\circ} \mathrm{C} \rightarrow$ rt, 2 steps, 65\%; (f) NIS, AgOTf, $\mathrm{CH}_{2} \mathrm{Cl}_{2}, 0{ }^{\circ} \mathrm{C}, 70 \%$, 8:1 $\alpha: \beta$; (g) $\mathrm{H}_{2}, \mathrm{Pd}(\mathrm{OH})_{2} / \mathrm{C}, \mathrm{CH}_{3} \mathrm{OH}$, rt, 82\%; (h) $\left(\mathrm{CH}_{3} \mathrm{O}\right)_{2} \mathrm{C}\left(\mathrm{CH}_{3}\right)_{2}$, acetone, $p$-TsOH, rt; (i) DIAD, $\mathrm{Ph}_{3} \mathrm{P}, \mathrm{THF}, 0{ }^{\circ} \mathrm{C} \rightarrow \mathrm{rt}$, 2 steps, 39\% 
General Methods. All reagents used were purchased from commercial sources and were used without further purification unless noted. Solvents used in reactions were purified by successive passage through columns of alumina and copper under nitrogen. Unless stated otherwise, all reactions were carried out under a positive pressure of argon and were monitored by TLC on silica gel G-25 UV $254(0.25 \mathrm{~mm})$. Spots were detected under UV light and/or by charring with $10 \% \mathrm{H}_{2} \mathrm{SO}_{4}$ in ethanol, or in acidified ethanolic anisaldehyde. Solvents were evaporated under reduced pressure and below $50{ }^{\circ} \mathrm{C}$ (water bath). Column chromatography was performed on Silica Gel $60(40-60 \mathrm{mM})$. The ratio between silica gel and crude product ranged from 100:1 to 20:1 (w/w). Iatrobeads refers to a beaded silica gel 6RS-8060, which is manufactured by Iatron Laboratories (Tokyo). Melting points were recorded on an eletrothermal melting point apparatus. ${ }^{1} \mathrm{H}$ NMR spectra were recorded on at 400, 500 or 600 $\mathrm{MHz}$, and chemical shifts are referenced to either TMS $\left(0.0, \mathrm{CDCl}_{3}\right)$ or $\mathrm{CD}_{3} \mathrm{OD}\left(4.78, \mathrm{CD}_{3} \mathrm{OD}\right)$. ${ }^{13} \mathrm{C}$ NMR APT spectra ${ }^{3}$ were recorded at 100 or $125 \mathrm{MHz}$, and ${ }^{13} \mathrm{C}$ chemical shifts are referenced to $\mathrm{CDCl}_{3}\left(77.23, \mathrm{CDCl}_{3}\right)$ or $\mathrm{CD}_{3} \mathrm{OD}\left(48.9, \mathrm{CD}_{3} \mathrm{OD}\right) . \quad{ }^{1} \mathrm{H} \mathrm{NMR}$ data are reported as though they are first order, and the peak assignments were made on the basis of 2D-NMR $\left({ }^{1} \mathrm{H}-{ }^{1} \mathrm{H}\right.$ COSY and HMQC) expeirments. $\quad$ ESI-MS spectra were recorded on samples suspended in THF or $\mathrm{CH}_{3} \mathrm{OH}$ and added $\mathrm{NaCl}$. Optical rotations were measured at $22 \pm 2{ }^{\circ} \mathrm{C}$ at the sodium D line $(589 \mathrm{~nm})$ and are in units of $\mathrm{deg} \cdot \mathrm{mL}(\mathrm{dm} \cdot \mathrm{g})^{-1}$.

\section{$n$-Octyl 2,3,5,6-tetra- $O$-acetyl- $\beta$-D-galactofuranosyl-( $1 \rightarrow 5)-2,3$-anhydro- $\alpha$-D-}

lyxofuranoside (S2). To a mixture of $22(250 \mathrm{mg}, 0.55 \mathrm{mmol}), \mathbf{4 1}^{1}$ (134 $\left.\mathrm{mg}, 0.55 \mathrm{mmol}\right)$ and 4 $\AA$ molecular sieves $(250 \mathrm{mg})$ in $\mathrm{CH}_{2} \mathrm{Cl}_{2}(15 \mathrm{~mL})$ was added $\mathrm{N}$-iodosuccinimide $(156 \mathrm{mg}, 0.66$ 
mmol) and silver triflate $(42 \mathrm{mg}, 0.16 \mathrm{mmol})$ in that order at $0{ }^{\circ} \mathrm{C}$. After stirring for 30 min at 0 ${ }^{\circ} \mathrm{C}$, the reaction mixture turned dark red and was neutralized by the addition of triethylamine. The solution was then diluted with $\mathrm{CH}_{2} \mathrm{Cl}_{2}(35 \mathrm{~mL})$ and filtered through Celite. The filtrate was washed with a sat. aq. $\mathrm{Na}_{2} \mathrm{~S}_{2} \mathrm{O}_{3}$ soln. $(3 \times 30 \mathrm{~mL})$, dried $\left(\mathrm{Na}_{2} \mathrm{SO}_{4}\right)$, and concentrated to give a crude residue that was purified by chromatography (3:1, hexanes: EtOAc) to provide $\mathbf{S 2}$ (281 $\mathrm{mg}, 89 \%)$ as a colorless oil. $\quad R_{f} 0.29\left(2: 1\right.$, hexanes:EtOAc); $[\alpha]_{\mathrm{D}}-21.4\left(c 0.6, \mathrm{CH}_{2} \mathrm{Cl}_{2}\right) ;{ }^{1} \mathrm{H} \mathrm{NMR}$ $\left(500 \mathrm{MHz}, \mathrm{CDCl}_{3}, \delta_{\mathrm{H}}\right) 5.37$ (ddd, $1 \mathrm{H}, J=7.0,4.4,3.8 \mathrm{~Hz}, \mathrm{H}-5$ '), 5.10-5.08 (m, $2 \mathrm{H}, \mathrm{H}-1^{\prime}, \mathrm{H}-2^{\prime}$ ), 5.02 (s, $1 \mathrm{H}, \mathrm{H}-1), 5.02-5.00$ (m, 1 H, H-3'), 4.34 (dd, 1 H, $J=11.8,4.4$ Hz, H-6'), 4.27 (dd, 1 H, $\left.J=5.9,3.8 \mathrm{~Hz}, \mathrm{H}-4^{\prime}\right), 4.21$ (dd, $1 \mathrm{H}, J=11.8,7.0 \mathrm{~Hz}, \mathrm{H}-6$ '), 4.19-4.16 (m, $\left.1 \mathrm{H}, \mathrm{H}-4\right), 3.78$ (dd, $1 \mathrm{H}, J=9.9,7.3 \mathrm{~Hz}, \mathrm{H}-5$ ), 3.75 (dd, $1 \mathrm{H}, J=2.9,0.7 \mathrm{~Hz}, \mathrm{H}-3$ ), 3.71 (ddd, $1 \mathrm{H}, J=9.5,6.7,6.7$ $\mathrm{Hz}$, octyl $\mathrm{OCH}_{2}$ ), 3.65 (dd, $\left.1 \mathrm{H}, J=9.9,5.9 \mathrm{~Hz}, \mathrm{H}-5\right), 3.65$ (d, $\left.1 \mathrm{H}, J=2.9 \mathrm{~Hz}, \mathrm{H}-2\right), 3.44$ (ddd, $1 \mathrm{H}, J=9.5,6.7,6.7 \mathrm{~Hz}$, octyl $\left.\mathrm{OCH}_{2}\right), 2.12\left(\mathrm{~s}, 3 \mathrm{H}\right.$, acyl $\left.\mathrm{CH}_{3}\right), 2.10\left(\mathrm{~s}, 3 \mathrm{H}\right.$, acyl $\left.\mathrm{CH}_{3}\right), 2.07(\mathrm{~s}, 3$ $\mathrm{H}$, acyl $\left.\mathrm{CH}_{3}\right), 2.05\left(\mathrm{~s}, 3 \mathrm{H}\right.$, acyl $\left.\mathrm{CH}_{3}\right), 1.60-1.53\left(\mathrm{~m}, 2 \mathrm{H}\right.$, octyl $\left.\mathrm{CH}_{2}\right), 1.35-1.22(\mathrm{~m}, 10 \mathrm{H}$, octyl $\left.\mathrm{CH}_{2}\right), 0.87\left(\mathrm{dd}, 3 \mathrm{H}, J=7.0,7.0 \mathrm{~Hz}\right.$, octyl $\left.\mathrm{CH}_{3}\right) ;{ }^{13} \mathrm{C} \mathrm{NMR}\left(125 \mathrm{MHz}, \mathrm{CDCl}_{3}, \delta_{\mathrm{C}}\right) 170.5(\mathrm{C}=\mathrm{O})$, $170.0(\mathrm{C}=\mathrm{O}), 169.9(\mathrm{C}=\mathrm{O}), 169.6(\mathrm{C}=\mathrm{O}), 105.5\left(\mathrm{C}-1^{\prime}\right), 101.2(\mathrm{C}-1), 81.3\left(\mathrm{C}-2^{\prime}\right), 80.0\left(\mathrm{C}-4^{\prime}\right)$, 76.5 (C-3'), 74.5 (C-4), 69.2 (C-5'), 68.6 (octyl $\mathrm{OCH}_{2}$ ), 65.4 (C-5), 62.6 (C-6'), 56.4 (C-2), 54.4 (C-3), 31.8 (octyl $\mathrm{CH}_{2}$ ), 29.6 (octyl $\mathrm{CH}_{2}$ ), 29.3 (octyl $\mathrm{CH}_{2}$ ), 29.2 (octyl $\mathrm{CH}_{2}$ ), 26.0 (octyl $\mathrm{CH}_{2}$ ), 22.6 (octyl $\left.\mathrm{CH}_{2}\right), 20.8\left(\right.$ acyl $\left.\mathrm{CH}_{3}\right), 20.7$ (acyl $\left.\mathrm{CH}_{3}\right), 20.66\left(\right.$ acyl $\left.\mathrm{CH}_{3}\right), 20.6\left(\right.$ acyl $\left.\mathrm{CH}_{3}\right), 14.1$ (octyl $\mathrm{CH}_{3}$ ). HRMS (ESI) calcd. for (M + Na) $\mathrm{C}_{27} \mathrm{H}_{42} \mathrm{O}_{13} \mathrm{Na}$ : 597.2518, found: 597.2518.

n-Octyl $\beta$-D-galactofuranosyl-(1 $\rightarrow$ 5)-2,3-anhydro- $\alpha$-D-lyxofuranoside $(\mathbf{S 3})$. To a solution of $\mathbf{S 2}(221 \mathrm{mg}, 0.39 \mathrm{mmol})$ in $\mathrm{CH}_{3} \mathrm{OH}(4 \mathrm{~mL})$ and $\mathrm{CH}_{2} \mathrm{Cl}_{2}(4 \mathrm{~mL})$ was added solid 
$\mathrm{NaOCH}_{3}$ until the $\mathrm{pH}$ was $\sim 10$. The solution was stirred at $\mathrm{rt}$ for $5 \mathrm{~h}$, followed by neutralization with acetic acid. The resulting mixture was concentrated and purified by chromatography (11:1, $\left.\mathrm{CH}_{2} \mathrm{Cl}_{2}: \mathrm{CH}_{3} \mathrm{OH}\right)$, to provide $\mathbf{S 3}(136 \mathrm{mg}, 87 \%)$ as a colorless oil. $R_{f} 0.38$ (10:1, $\left.\mathrm{CH}_{2} \mathrm{Cl}_{2}: \mathrm{CH}_{3} \mathrm{OH}\right) ;[\alpha]_{\mathrm{D}}-28.9\left(c 0.2, \mathrm{CH}_{3} \mathrm{OH}\right) ;{ }^{1} \mathrm{H} \mathrm{NMR}\left(500 \mathrm{MHz}, \mathrm{CD}_{3} \mathrm{OD}, \delta_{\mathrm{H}}\right) 5.00(\mathrm{~s}, 1 \mathrm{H}$, H-1), 4.91 (d, 1 H, $J=1.7 \mathrm{~Hz}, \mathrm{H}-1$ '), 4.13 (dd, $1 \mathrm{H}, J=6.1,6.0 \mathrm{~Hz}, \mathrm{H}-4), 4.00$ (dd, $1 \mathrm{H}, J=6.3$, $\left.3.7 \mathrm{~Hz}, \mathrm{H}-3^{\prime}\right), 3.97$ (dd, $1 \mathrm{H}, J=3.7,1.7 \mathrm{~Hz}, \mathrm{H}-2^{\prime}$ ), 3.96 (dd, $1 \mathrm{H}, J=6.3,3.1 \mathrm{~Hz}, \mathrm{H}-4^{\prime}$ ), 3.86-3.82 (m, 2 H, H-3, H-5), 3.73-3.68 (m, 2 H, H-5', octyl $\mathrm{OCH}_{2}$ ), 3.64 (d, $1 \mathrm{H}, J=2.9 \mathrm{~Hz}$, H-2), 3.63-3.56 (m, 3 H, H-6' × 2, H-5), 3.46 (ddd, $1 \mathrm{H}, J=9.6,6.6,6.6 \mathrm{~Hz}$, octyl $\left.\mathrm{OCH}_{2}\right)$, 1.60-1.52 (m, $2 \mathrm{H}$, octyl $\left.\mathrm{CH}_{2}\right), 1.34-1.22\left(\mathrm{~m}, 10 \mathrm{H}\right.$, octyl $\left.\mathrm{CH}_{2}\right), 0.87(\mathrm{dd}, 3 \mathrm{H}, J=6.9,6.9 \mathrm{~Hz}$, octyl $\left.\mathrm{CH}_{3}\right) ;{ }^{13} \mathrm{C}$ NMR $\left(125 \mathrm{MHz}, \mathrm{CD}_{3} \mathrm{OD}, \delta_{\mathrm{C}}\right) 109.7$ (C-1'), 102.7 (C-1), 84.7 (C-4'), 83.2 (C-2'), 78.8 (C-3’), 76.4 (C-4), 72.7 (C-5'), 69.4 (octyl $\mathrm{OCH}_{2}$ ), 67.1 (C-5), 64.5 (C-6'), 57.1 (C-2), 55.3 (C-3), 33.0 (octyl $\mathrm{CH}_{2}$ ), 32.6 (octyl $\mathrm{CH}_{2}$ ), 30.8 (octyl $\mathrm{CH}_{2}$ ), 30.5 (octyl $\mathrm{CH}_{2}$ ), 30.4 (octyl $\mathrm{CH}_{2}$ ), 27.2 (octyl $\mathrm{CH}_{2}$ ), 14.4 (octyl $\mathrm{CH}_{3}$ ). HRMS (ESI) calcd. for $(\mathrm{M}+\mathrm{Na}) \mathrm{C}_{19} \mathrm{H}_{34} \mathrm{O}_{9} \mathrm{Na}$ : 429.2095, found: 429.2093 .

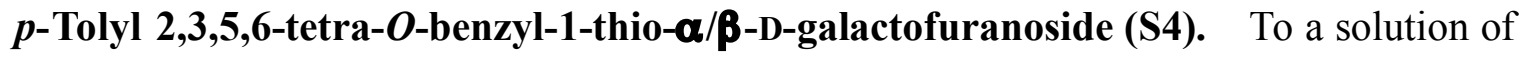
an anomeric mixture of 23 and $24(643 \mathrm{mg}, 2.22 \mathrm{mmol})$ in DMF $(15 \mathrm{~mL})$ at $0{ }^{\circ} \mathrm{C}$ was added $\mathrm{NaH}$ ( $0.585 \mathrm{~g}, 14.6 \mathrm{mmol}, 60 \%$ dispersion in oil) and the solution was stirred for $10 \mathrm{~min}$. Benzyl bromide $(1.3 \mathrm{~mL}, 11.1 \mathrm{mmol})$ was added dropwise and the solution was stirred for $5 \mathrm{~h}$ at $\mathrm{rt}$. The reaction mixture was quenched by adding a few drops of $\mathrm{CH}_{3} \mathrm{OH}$, diluted with $\mathrm{CH}_{2} \mathrm{Cl}_{2}(50$ $\mathrm{mL})$, and washed with a sat. aq. $\mathrm{NaHCO}_{3}$ soln. $(3 \times 40 \mathrm{~mL})$ and water $(3 \times 40 \mathrm{~mL})$. The organic layer was dried $\left(\mathrm{Na}_{2} \mathrm{SO}_{4}\right)$, filtered, concentrated, and the resulting residue was purified 
by chromatography (7:1, hexanes:EtOAc) to provide $\mathbf{S} 4(1.402 \mathrm{~g}, 98 \%)$ as a colorless oil. $\quad R_{f}$ 0.50 (6:1, hexanes: EtOAc); ${ }^{1} \mathrm{H}$ NMR $\left(500 \mathrm{MHz}, \mathrm{CDCl}_{3}, \delta_{\mathrm{H}}\right) 7.51-7.47(\mathrm{~m}, 1 \mathrm{H}, \mathrm{Ar}), 7.43-7.23$ (m, $21 \mathrm{H}, \mathrm{Ar}), 7.13-7.09$ (m, $2 \mathrm{H}, \mathrm{Ar}), 5.67$ (d, $0.4 \mathrm{H}, J=4.7 \mathrm{~Hz}, \mathrm{H}-1 \alpha$ ), 5.59 (d, $0.6 \mathrm{H}, J=2.8$

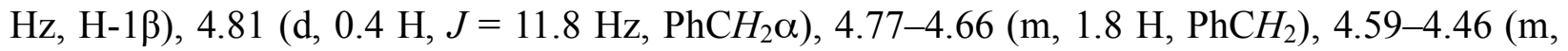
$5.2 \mathrm{H}, \mathrm{PhCH}_{2}$ ), 4.39 (dd, 0.6 H, $J=7.1,3.4 \mathrm{~Hz}, \mathrm{H}-4 \beta$ ), 4.36 (d, $0.6 \mathrm{H}, J=11.7 \mathrm{~Hz}, \mathrm{PhCH}_{2} \beta$ ),

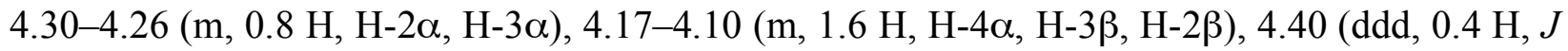
$=6.5,6.5,4.6 \mathrm{~Hz}, \mathrm{H}-5 \alpha), 3.86(\mathrm{ddd}, 0.6 \mathrm{H}, J=5.9,5.9,3.4 \mathrm{~Hz}, \mathrm{H}-5 \beta), 3.78-3.69$ (m, $1.6 \mathrm{H}$,

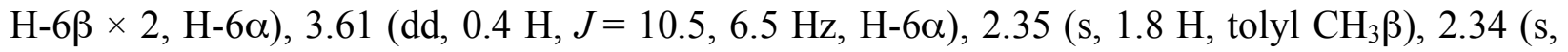
$1.2 \mathrm{H}$, tolyl $\left.\mathrm{CH}_{3} \alpha\right) ;{ }^{13} \mathrm{C}$ NMR $\left(125 \mathrm{MHz}, \mathrm{CDCl}_{3}, \delta_{\mathrm{C}}\right) 138.9$ (Ar $\left.\alpha\right), 138.4(\operatorname{Ar} \beta), 138.33$ (Ar $\alpha$ ), $138.3(\operatorname{Ar} \beta), 137.84(\operatorname{Ar} \alpha), 137.8(\operatorname{Ar} \beta), 137.5(\operatorname{Ar} \alpha), 137.4(\operatorname{Ar} \beta), 137.3(\operatorname{Ar} \alpha), 136.8(\operatorname{Ar} \beta)$, 132.1 (Ar $\beta), 131.8(\operatorname{Ar} \alpha), 131.3(\operatorname{Ar} \alpha), 130.9(\operatorname{Ar} \beta), 129.7(\operatorname{Ar} \alpha), 129.6(\operatorname{Ar} \beta), 128.5-127.4(\operatorname{Ar})$,

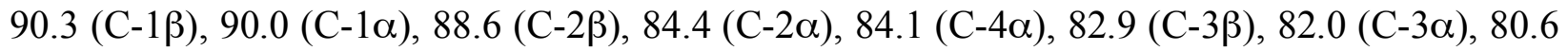

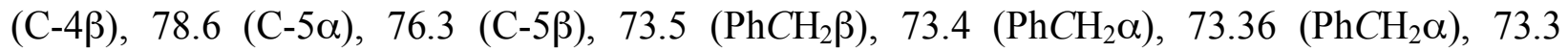
( $\left.\mathrm{PhCH}_{2} \beta\right), 72.4\left(\mathrm{PhCH}_{2} \alpha\right), 72.2\left(\mathrm{PhCH}_{2} \beta\right), 72.0\left(\mathrm{PhCH}_{2} \beta\right), 71.9\left(\mathrm{PhCH}_{2} \alpha\right), 70.8(\mathrm{C}-6 \alpha \beta), 21.14$ (tolyl $\mathrm{CH}_{3} \beta$ ), 21.1 (tolyl $\mathrm{CH}_{3} \alpha$ ). HRMS (ESI) calcd. for $(\mathrm{M}+\mathrm{Na}) \mathrm{C}_{41} \mathrm{H}_{42} \mathrm{O}_{5} \mathrm{SNa}$ : 669.2645, found: 669.2642 .

$\boldsymbol{n}$-Octyl 2,3,5,6-tetra-O-benzyl- $\boldsymbol{\alpha}$-D-galactofuranoside (S5). To a mixture of S4 (490 $\mathrm{mg}, 0.76 \mathrm{mmol}), n$-octanol $(0.14 \mathrm{~mL}, 0.91 \mathrm{mmol})$ and $4 \AA$ molecular sieves $(0.5 \mathrm{~g})$ in $\mathrm{CH}_{2} \mathrm{Cl}_{2}(20$ $\mathrm{mL}$ ) was added $N$-iodosuccinimide $(216 \mathrm{mg}, 0.91 \mathrm{mmol})$ at $0{ }^{\circ} \mathrm{C}$. After stirring for $10 \mathrm{~min}$ at 0 ${ }^{\circ} \mathrm{C}$, the reaction mixture was neutralized with triethylamine. The resulting solution was then diluted with $\mathrm{CH}_{2} \mathrm{Cl}_{2}(30 \mathrm{~mL})$ and filtered through Celite. The filtrate was washed with a sat. aq. 
$\mathrm{Na}_{2} \mathrm{~S}_{2} \mathrm{O}_{3}$ soln. $(3 \times 30 \mathrm{~mL})$, dried $\left(\mathrm{Na}_{2} \mathrm{SO}_{4}\right)$, and concentrated to give a crude residue that was purified by chromatography (10:1, hexanes:EtOAc) to give S5 (307 $\mathrm{mg}, 62 \%)$ and the corresponding $\beta$-glycoside anomer (82 $\mathrm{mg}, 17 \%) ; R_{f} 0.47$ (6:1, hexanes:EtOAc)) as colorless oils. Data for major isomer: S5: $R_{f} 0.37$ (6:1, hexanes:EtOAc); $[\alpha]_{\mathrm{D}}+27.7\left(c \quad 0.8, \mathrm{CH}_{2} \mathrm{Cl}_{2}\right) ;{ }^{1} \mathrm{H} \mathrm{NMR}$ $\left(500 \mathrm{MHz}, \mathrm{CDCl}_{3}, \delta_{\mathrm{H}}\right) 7.41-7.20(\mathrm{~m}, 20 \mathrm{H}, \mathrm{Ar}), 4.90(\mathrm{~d}, 1 \mathrm{H}, J=4.3 \mathrm{~Hz}, \mathrm{H}-1), 4.76(\mathrm{~d}, 2 \mathrm{H}, J=$ $\left.12.1 \mathrm{~Hz}, \mathrm{PhCH}_{2}\right), 4.66$ (d, $\left.2 \mathrm{H}, J=11.6 \mathrm{~Hz}, \mathrm{PhCH}_{2}\right), 4.58\left(\mathrm{~d}, 1 \mathrm{H}, J=11.6 \mathrm{~Hz}, \mathrm{PhCH}_{2}\right), 4.52(\mathrm{~d}$, $\left.2 \mathrm{H}, J=11.8 \mathrm{~Hz}, \mathrm{PhCH}_{2}\right), 4.48\left(\mathrm{~d}, 1 \mathrm{H}, J=12.1 \mathrm{~Hz}, \mathrm{PhCH}_{2}\right), 4.31$ (dd, $\left.1 \mathrm{H}, J=7.5,7.0 \mathrm{~Hz}, \mathrm{H}-3\right)$, 4.07 (dd, $1 \mathrm{H}, J=7.5,4.3 \mathrm{~Hz}, \mathrm{H}-2), 3.99$ (dd, $1 \mathrm{H}, J=7.0,6.6 \mathrm{~Hz}, \mathrm{H}-4), 3.75-3.65$ (m, $3 \mathrm{H}, \mathrm{H}-5$, H-6, octyl $\mathrm{OCH}_{2}$ ), 3.60 (dd, $\left.1 \mathrm{H}, J=10.4,6.3 \mathrm{~Hz}, \mathrm{H}-6\right), 3.33$ (ddd, $1 \mathrm{H}, J=9.6,7.0,7.0 \mathrm{~Hz}$, octyl $\left.\mathrm{OCH}_{2}\right), 1.64-1.52\left(\mathrm{~m}, 2 \mathrm{H}\right.$, octyl $\left.\mathrm{CH}_{2}\right), 1.36-1.24\left(\mathrm{~m}, 10 \mathrm{H}\right.$, octyl $\left.\mathrm{CH}_{2}\right), 0.91$ (dd, $3 \mathrm{H}, J=$ 7.0, 7.0 Hz, octyl $\left.\mathrm{CH}_{3}\right) ;{ }^{13} \mathrm{C} \mathrm{NMR}\left(125 \mathrm{MHz}, \mathrm{CDCl}_{3}, \delta_{\mathrm{C}}\right) 138.9(\mathrm{Ar}), 138.3(\mathrm{Ar} \times 2), 137.8(\mathrm{Ar})$, $128.4(\mathrm{Ar} \times 2), 128.32(\mathrm{Ar} \times 2), 128.3(\mathrm{Ar} \times 2), 128.2(\mathrm{Ar} \times 2), 128.1(\mathrm{Ar} \times 2), 127.9(\mathrm{Ar})$, $127.85(\mathrm{Ar} \times 2), 127.8(\mathrm{Ar} \times 2), 127.6(\mathrm{Ar}), 127.55(\mathrm{Ar} \times 2), 127.5(\mathrm{Ar}), 127.4(\mathrm{Ar}), 100.0(\mathrm{C}-1)$, 84.2 (C-2), 81.3 (C-3), 80.4 (C-4), $79.9(\mathrm{C}-5), 73.4\left(\mathrm{PhCH}_{2}\right), 73.1\left(\mathrm{PhCH}_{2}\right), 72.35\left(\mathrm{PhCH}_{2}\right), 72.3$ ( $\mathrm{PhCH}_{2}$ ), 70.5 (C-6), 68.0 (octyl $\mathrm{OCH}_{2}$ ), 31.9 (octyl $\mathrm{CH}_{2}$ ), 29.54 (octyl $\mathrm{CH}_{2}$ ), 29.5 (octyl $\mathrm{CH}_{2}$ ), 29.3 (octyl $\mathrm{CH}_{2}$ ), 26.3 (octyl $\mathrm{CH}_{2}$ ), 22.7 (octyl $\mathrm{CH}_{2}$ ), 14.1 (octyl $\mathrm{CH}_{3}$ ). HRMS (ESI) calcd. for $(\mathrm{M}+\mathrm{Na}) \mathrm{C}_{42} \mathrm{H}_{52} \mathrm{O}_{6} \mathrm{Na}$ : 675.3656, found: 675.3657 .

$\boldsymbol{n}$-Octyl $\boldsymbol{\alpha}$-D-galactofuranoside (S6). To a solution of $\mathbf{S 5}(201 \mathrm{mg}, 0.31 \mathrm{mmol})$ in methanol $(2.0 \mathrm{~mL})$ was added $20 \%$ palladium hydroxide on carbon and the reaction was stirred under positive pressure of hydrogen for $12 \mathrm{~h}$. The resulting mixture was filtered through Celite and concentrated to give a crude residue that was purified by chromatography (11:1, 
$\left.\mathrm{CH}_{2} \mathrm{Cl}_{2}: \mathrm{CH}_{3} \mathrm{OH}\right)$ to yield $\mathbf{S 6}(76 \mathrm{mg}, 85 \%)$ as a white foam. $R_{f} 0.36\left(10: 1, \mathrm{CH}_{2} \mathrm{Cl}_{2}: \mathrm{CH}_{3} \mathrm{OH}\right)$; $[\alpha]_{\mathrm{D}}+83.5\left(c 0.2, \mathrm{CH}_{3} \mathrm{OH}\right) ;{ }^{1} \mathrm{H} \mathrm{NMR}\left(500 \mathrm{MHz}, \mathrm{CD}_{3} \mathrm{OD}, \delta_{\mathrm{H}}\right) 4.83(\mathrm{~d}, 1 \mathrm{H}, J=4.6 \mathrm{~Hz}, \mathrm{H}-1)$, 4.07 (dd, $1 \mathrm{H}, J=7.8,7.0 \mathrm{~Hz}, \mathrm{H}-3$ ), 3.93 (dd, $1 \mathrm{H}, J=7.8,4.6 \mathrm{~Hz}, \mathrm{H}-2$ ), 3.78 (ddd, $1 \mathrm{H}, J=9.6$, 6.7, $6.7 \mathrm{~Hz}$, octyl $\mathrm{OCH}_{2}$ ), 3.71 (dd, $\left.1 \mathrm{H}, J=7.0,5.2 \mathrm{~Hz}, \mathrm{H}-4\right), 3.64-3.59$ (m, $\left.2 \mathrm{H}, \mathrm{H}-5, \mathrm{H}-6\right)$, 3.57-3.51 (m, $1 \mathrm{H}, \mathrm{H}-6), 3.45$ (ddd, $1 \mathrm{H}, J=9.6,6.7,6.7 \mathrm{~Hz}$, octyl $\left.\mathrm{OCH}_{2}\right), 1.64-1.57$ (m, $2 \mathrm{H}$, octyl $\left.\mathrm{CH}_{2}\right), 1.40-1.24\left(\mathrm{~m}, 10 \mathrm{H}\right.$, octyl $\left.\mathrm{CH}_{2}\right), 0.91-0.86$ (m, $3 \mathrm{H}$, octyl $\left.\mathrm{CH}_{3}\right) ;{ }^{13} \mathrm{C} \mathrm{NMR}(125 \mathrm{MHz}$, $\left.\mathrm{CD}_{3} \mathrm{OD}, \delta_{\mathrm{C}}\right) 102.9(\mathrm{C}-1), 83.6(\mathrm{C}-4), 78.9(\mathrm{C}-2), 76.4(\mathrm{C}-3), 74.5$ (C-5), 69.8 (octyl $\left.\mathrm{OCH}_{2}\right), 64.2$ (C-6), 33.0 (octyl $\mathrm{CH}_{2}$ ), 30.8 (octyl $\mathrm{CH}_{2}$ ), 30.6 (octyl $\mathrm{CH}_{2}$ ), 30.4 (octyl $\mathrm{CH}_{2}$ ), 27.2 (octyl $\mathrm{CH}_{2}$ ), 23.7 (octyl $\mathrm{CH}_{2}$ ), 14.4 (octyl $\mathrm{CH}_{3}$ ). HRMS (ESI) calcd. for $(\mathrm{M}+\mathrm{Na}) \mathrm{C}_{14} \mathrm{H}_{28} \mathrm{O}_{6} \mathrm{Na}$ : 315.1778, found: 315.1778 .

\section{$n$-Octyl 2,3,5,6-tetra- $O$-benzyl- $\alpha$ / $\beta$-D-galactofuranosyl-(1 $\rightarrow 5)-2,3$-anhydro- $\alpha$-D-}

lyxofuranoside (S7). To a mixture of $\mathbf{S 4}(490 \mathrm{mg}, 0.76 \mathrm{mmol}), \mathbf{4 1}^{1}(186 \mu \mathrm{L}, 0.76 \mathrm{mmol})$ and 4 $\AA$ molecular sieves $(0.5 \mathrm{~g})$ in $\mathrm{CH}_{2} \mathrm{Cl}_{2}(20 \mathrm{~mL})$ was added $N$-iodosuccinimide $(216 \mathrm{mg}, 0.91$ $\mathrm{mmol}$ ) and silver triflate $(59 \mathrm{mg}, 0.23 \mathrm{mmol})$ in that order at $0{ }^{\circ} \mathrm{C}$. After stirring for $30 \mathrm{~min}$ at 0 ${ }^{\circ} \mathrm{C}$, the reaction mixture was neutralized with triethylamine, diluted with $\mathrm{CH}_{2} \mathrm{Cl}_{2}(30 \mathrm{~mL})$ and then filtered through Celite. The filtrate was washed with a sat. aq. $\mathrm{Na}_{2} \mathrm{~S}_{2} \mathrm{O}_{3}$ soln. $(3 \times 30 \mathrm{~mL})$, dried $\left(\mathrm{Na}_{2} \mathrm{SO}_{4}\right)$, and concentrated to give a crude residue that was purified by chromatography (6:1, hexanes:EtOAc) to give an $\alpha / \beta$ mixture of $\mathbf{S 7}(405 \mathrm{mg}, 70 \%)$ as a colorless oil in an $8: 1 \alpha: \beta$ ratio. NMR data only for $\alpha$-anomer: $R_{f} 0.27$ (6:1, hexanes:EtOAc); ${ }^{1} \mathrm{H} \mathrm{NMR}\left(500 \mathrm{MHz}, \mathrm{CDCl}_{3}\right.$, $\left.\delta_{\mathrm{H}}\right) 7.42-7.25(\mathrm{~m}, 20 \mathrm{H}, \mathrm{Ar}), 5.03(\mathrm{~s}, 1 \mathrm{H}, \mathrm{H}-1), 4.94$ (d, $1 \mathrm{H}, J=4.4 \mathrm{~Hz}, \mathrm{H}-1$ ') $), 4.82-4.49$ (m, 8 H, PhCH $H_{2}$ ), 4.39 (dd, $1 \mathrm{H}, J=7.7,7.3 \mathrm{~Hz}, \mathrm{H}-3$ '), 4.17 (dd, $1 \mathrm{H}, J=8.4,5.0 \mathrm{~Hz}, \mathrm{H}-4$ ), 4.11 (dd, 1 
H, $\left.J=7.7,4.4 \mathrm{~Hz}, \mathrm{H}-2^{\prime}\right), 4.03$ (dd, $1 \mathrm{H}, J=7.3,4.9 \mathrm{~Hz}, \mathrm{H}-4$ '), 3.88 (dd, $1 \mathrm{H}, J=9.8,5.0 \mathrm{~Hz}$, H-5), 3.77-3.66 (m, 4 H, H-5', H-6' × 2, octyl $\mathrm{OCH}_{2}$ ), 3.75 (d, 1 H, $J=2.8$ Hz, H-3), 3.63 (d, 1 H, $J=2.8 \mathrm{~Hz}, \mathrm{H}-2), 3.55$ (dd, $1 \mathrm{H}, J=9.8,8.4 \mathrm{~Hz}, \mathrm{H}-5$ ), 3.45 (ddd, $1 \mathrm{H}, J=9.4,6.7,6.7 \mathrm{~Hz}$, octyl $\left.\mathrm{OCH}_{2}\right), 1.64-1.58\left(\mathrm{~m}, 2 \mathrm{H}\right.$, octyl $\left.\mathrm{CH}_{2}\right), 1.39-1.27\left(\mathrm{~m}, 10 \mathrm{H}\right.$, octyl $\left.\mathrm{CH}_{2}\right), 0.95-0.91$ (dd, $3 \mathrm{H}$, $J=7.0,7.0 \mathrm{~Hz}$, octyl $\left.\mathrm{CH}_{3}\right) ;{ }^{13} \mathrm{C} \mathrm{NMR}\left(125 \mathrm{MHz}, \mathrm{CDCl}_{3}, \delta_{\mathrm{C}}\right) 138.7(\mathrm{Ar}), 138.31(\mathrm{Ar}), 138.3(\mathrm{Ar})$, 137.8 (Ar), 128.7 (Ar), 128.4 (Ar), 128.3 (Ar), 128.1 (Ar), 128.0 (Ar), 127.9 (Ar), 127.8 (Ar), 127.6 (Ar), 127.5 (Ar), 101.1 (C-1), 100.4 (C-1'), 84.3 (C-2'), 80.33 (C-3'), 80.3 (C-4'), 78.6 (C-5'), 74.5 (C-4), $73.5\left(\mathrm{PhCH}_{2}\right), 73.2\left(\mathrm{PhCH}_{2}\right), 72.5\left(\mathrm{PhCH}_{2}\right), 72.4\left(\mathrm{PhCH}_{2}\right), 70.5(\mathrm{C}-6$ ') 68.5 (octyl $\mathrm{OCH}_{2}$ ), 66.0 (C-5), 56.7 (C-2), 54.7 (C-3), 31.9 (octyl $\mathrm{CH}_{2}$ ), 29.7 (octyl $\mathrm{CH}_{2}$ ), 29.4 (octyl $\mathrm{CH}_{2}$ ), 29.3 (octyl $\mathrm{CH}_{2}$ ), 26.1 (octyl $\mathrm{CH}_{2}$ ), 22.7 (octyl $\mathrm{CH}_{2}$ ), 14.2 (octyl $\mathrm{CH}_{3}$ ). HRMS (ESI) calcd. for $(\mathrm{M}+\mathrm{Na}) \mathrm{C}_{47} \mathrm{H}_{58} \mathrm{O}_{9} \mathrm{Na}: 789.3973$, found: 789.3973 .

n-Octyl $\alpha$-D-galactofuranosyl-(1 $\rightarrow$ 5)-2,3-anhydro- $\alpha$-D-lyxofuranoside $(\mathrm{S8}) . \quad$ To a solution of $\mathbf{S 7}(140 \mathrm{mg}, 0.18 \mathrm{mmol})$ in $\mathrm{CH}_{3} \mathrm{OH}(2.0 \mathrm{~mL})$ was added $20 \%$ palladium hydroxide on carbon and the reaction was stirred under positive pressure of hydrogen for $12 \mathrm{~h}$. The resulting mixture was filtered through Celite and concentrated to give a crude residue that was purified by chromatography $\left(11: 1, \mathrm{CH}_{2} \mathrm{Cl}_{2}: \mathrm{CH}_{3} \mathrm{OH}\right)$ to yield $\mathbf{S 8}(61 \mathrm{mg}, 82 \%)$ as a colorless oil. $R_{f} 0.38$ $\left(10: 1, \mathrm{CH}_{2} \mathrm{Cl}_{2}: \mathrm{CH}_{3} \mathrm{OH}\right) ;[\alpha]_{\mathrm{D}}+98.7\left(c 0.2, \mathrm{CH}_{3} \mathrm{OH}\right) ;{ }^{1} \mathrm{H} \mathrm{NMR}\left(500 \mathrm{MHz}, \mathrm{CD}_{3} \mathrm{OD}, \delta_{\mathrm{H}}\right) 5.02(\mathrm{~s}, 1$ H, H-1), 4.87 (d, $1 \mathrm{H}, J=4.7 \mathrm{~Hz}, \mathrm{H}-1$ '), 4.19 (dd, $1 \mathrm{H}, J=6.5,5.9 \mathrm{~Hz}, \mathrm{H}-4$ ), 4.16 (dd, $1 \mathrm{H}, J=$ 8.1, 7.3 Hz, H-3'), 3.95 (dd, 1 H, $J=8.1,4.7$ Hz, H-2'), 3.88 (d, 1 H, $J=2.9$ Hz, H-3), 3.86 (dd, $1 \mathrm{H}, J=10.1,6.5 \mathrm{~Hz}, \mathrm{H}-5), 3.75$ (dd, $1 \mathrm{H}, J=7.3,4.0 \mathrm{~Hz}, \mathrm{H}-4$ '), 3.74-3.68 (m, $2 \mathrm{H}, \mathrm{H}-5$, octyl $\mathrm{OCH}_{2}$ ), 3.65 (d, $\left.1 \mathrm{H}, J=2.9 \mathrm{~Hz}, \mathrm{H}-2\right), 3.64-3.54$ (m, $3 \mathrm{H}, \mathrm{H}-6$ ' × 2, H-5'), 3.46 (ddd, $1 \mathrm{H}, J=$ 
9.5, 6.6, 6.6 Hz, octyl $\left.\mathrm{OCH}_{2}\right), 1.61-1.52\left(\mathrm{~m}, 2 \mathrm{H}\right.$, octyl $\left.\mathrm{CH}_{2}\right), 1.34-1.23\left(\mathrm{~m}, 10 \mathrm{H}\right.$, octyl $\left.\mathrm{CH}_{2}\right)$, 0.91-0.83 (m, $3 \mathrm{H}, J=7.0,7.0 \mathrm{~Hz}$, octyl $\left.\mathrm{CH}_{3}\right) ;{ }^{13} \mathrm{C} \mathrm{NMR}\left(125 \mathrm{MHz}, \mathrm{CD}_{3} \mathrm{OD}, \delta_{\mathrm{C}}\right) 103.2\left(\mathrm{C}-1^{\prime}\right)$, 102.7 (C-1), 83.4 (C-4'), 78.9 (C-2’), 76.3 (C-4), 75.7 (C-3’), 73.6 (C-5'), 69.5 (octyl OCH2), 68.3 (C-5), 64.3 (C-6’), 57.3 (C-2), 55.2 (C-3), 33.0 (octyl $\mathrm{CH}_{2}$ ), 30.8 (octyl $\mathrm{CH}_{2}$ ), 30.5 (octyl $\mathrm{CH}_{2}$ ), 30.4 (octyl $\mathrm{CH}_{2}$ ), 27.2 (octyl $\mathrm{CH}_{2}$ ), 23.7 (octyl $\mathrm{CH}_{2}$ ), 14.4 (octyl $\mathrm{CH}_{3}$ ). HRMS (ESI) calcd. for $(\mathrm{M}+\mathrm{Na}) \mathrm{C}_{19} \mathrm{H}_{34} \mathrm{O}_{9} \mathrm{Na}: 429.2095$, found: 429.2094 .

2-deoxy-2-thiotolyl-D-idose (26). A solution of 13 (62 $\mathrm{mg}, 0.2 \mathrm{mmol})$ in $50 \%$ aqueous acetic acid $(20 \mathrm{~mL})$ was stirred for $12 \mathrm{~h}$. Concentration of the solution and chromatography (10:1, $\left.\mathrm{CH}_{2} \mathrm{Cl}_{2}: \mathrm{CH}_{3} \mathrm{OH}\right)$ gave $26(38 \mathrm{mg}, 72 \%)$ as a colorless oil. $R_{f} 0.19\left(10: 1, \mathrm{CH}_{2} \mathrm{Cl}_{2}: \mathrm{CH}_{3} \mathrm{OH}\right)$; ${ }^{1} \mathrm{H}$ NMR (500 MHz, CD $\left.\mathrm{CD}_{3} \delta_{\mathrm{H}}\right)$ 7.42-7.35 (m, $\left.2 \mathrm{H}, \mathrm{Ar}\right), 7.17-7.09$ (m, $\left.2 \mathrm{H}, \mathrm{Ar}\right), 5.51$ (d, 0.23 $\mathrm{H}, J=4.8 \mathrm{~Hz}, \mathrm{H}-1 \beta$-furanose), 5.25 (d, $0.13 \mathrm{H}, J=2.6 \mathrm{~Hz}, \mathrm{H}-1 \alpha$-pyranose), 5.17 (d, $0.48 \mathrm{H}, J$ $=2.1 \mathrm{~Hz}, \mathrm{H}-1 \alpha$-furanose), $5.08(\mathrm{~d}, 0.16 \mathrm{H}, J=4.1 \mathrm{~Hz}, \mathrm{H}-1 \beta$-pyranose), 4.37 (dd, $0.23 \mathrm{H}, J=$ 6.2, $6.2 \mathrm{~Hz}, \mathrm{H}-3 \beta$-furanose), 4.24 (dd, $0.23 \mathrm{H}, J=6.2,3.5 \mathrm{~Hz}, \mathrm{H}-4 \beta$-furanose), $4.21-4.18$ (m, $0.16 \mathrm{H}), 4.18-4.13(\mathrm{~m}, 1.13 \mathrm{H}), 3.98-3.93(\mathrm{~m}, 0.64 \mathrm{H}), 3.89$ (m, $0.23 \mathrm{H}, \mathrm{H}-5$-furanose), 3.81-3.55 (m, 2.61 H), $3.52(\mathrm{dd}, 0.48 \mathrm{H}, J=2.8,2.1 \mathrm{~Hz}, \mathrm{H}-2 \alpha$-furanose), 3.24 (dd, $0.13 \mathrm{H}, J=$

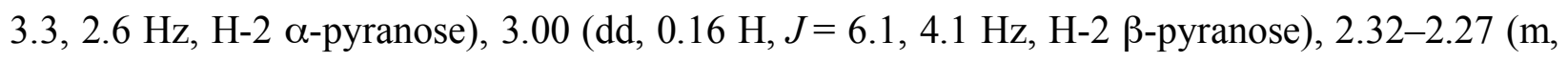
$3 \mathrm{H}$, tolyl $\left.\mathrm{CH}_{3}\right) ;{ }^{13} \mathrm{C}$ NMR (125 MHz, $\mathrm{CD}_{3} \mathrm{OD}, \delta_{\mathrm{C}}$ ) 138.6-129.8 (Ar), 103.5 (C-1 $\alpha$-furanose), 97.8 (C-1 $\beta$-furanose), 95.3 (C-1 $\beta$-pyranose), 94.1 (C-1 $\alpha$-pyranose), 82.6, 79.2, 77.8, 77.3, 77.0, $76.9,72.5,72.2,72.19,72.15,72.1,71.9,71.1,70.9,64.4,64.3,64.2,63.0,61.8,61.0,60.6,56.7$, 54.2, 21.1, 21.0. HRMS (ESI) calcd. for $(\mathrm{M}+\mathrm{Na}) \mathrm{C}_{13} \mathrm{H}_{18} \mathrm{O}_{5} \mathrm{SNa}$ : 309.0769, found: 309.0767 . 
n-Octyl 2,3-anhydro-5,6-O-isopropylidene- $\alpha$-D-gulofuranoside (34). To a solution of S6 (61 mg, $0.21 \mathrm{mmol})$ and 2,2-dimethoxypropane $(0.21 \mathrm{~mL}, 1.7 \mathrm{mmol})$ in acetone $(1.5 \mathrm{~mL})$ was added $p$-toluenesufonic acid (0.4 mg, cat.) at rt. The solution was stirred for $4 \mathrm{~h}$, and neutralized with triethylamine. The mixture was concentrated and the resulting oil was dissolved in THF (2.0 mL), followed by the addition of $\mathrm{PPh}_{3}(67 \mathrm{mg}, 0.25 \mathrm{mmol})$. DIAD (51 $\mu \mathrm{L}, 0.25 \mathrm{mmol}$ ) was then added dropwise at $0{ }^{\circ} \mathrm{C}$ and the reaction mixture was warmed to $\mathrm{rt}$ and stirred for $30 \mathrm{~min}$. The resulting solution was concentrated and the residue was purified by chromatography (4:1, hexanes:EtOAc) to yield 34 (43 mg, 65\% over two steps) as a white foam. $R_{f} 0.26$ (4:1, hexanes:EtOAc); $[\alpha]_{\mathrm{D}}+44.9\left(c 1.0, \mathrm{CH}_{2} \mathrm{Cl}_{2}\right) ;{ }^{1} \mathrm{H} \mathrm{NMR}\left(500 \mathrm{MHz}, \mathrm{CDCl}_{3}, \delta_{\mathrm{H}}\right) 5.08$ (s, $1 \mathrm{H}, \mathrm{H}-1$ ), 4.15 (ddd, $1 \mathrm{H}, J=6.7,6.6,6.6 \mathrm{~Hz}, \mathrm{H}-5), 4.11$ (dd, $1 \mathrm{H}, J=8.5,6.6 \mathrm{~Hz}, \mathrm{H}-6), 3.98$ (dd, $1 \mathrm{H}, J=8.5,6.7 \mathrm{~Hz}, \mathrm{H}-6), 3.88$ (dd, $1 \mathrm{H}, J=6.6,0.7 \mathrm{~Hz}, \mathrm{H}-4), 3.82$ (ddd, $1 \mathrm{H}, J=9.6,6.8$, $6.8 \mathrm{~Hz}$, octyl $\left.\mathrm{OCH}_{2}\right), 3.66(\mathrm{~d}, 1 \mathrm{H}, J=3.0 \mathrm{~Hz}, \mathrm{H}-2), 3.58$ (dd, $\left.1 \mathrm{H}, J=3.0,0.7 \mathrm{~Hz}, \mathrm{H}-3\right), 3.55$ (ddd, $1 \mathrm{H}, J=9.6,6.8,6.8 \mathrm{~Hz}$, octyl $\left.\mathrm{OCH}_{2}\right), 1.64-1.58\left(\mathrm{~m}, 2 \mathrm{H}\right.$, octyl $\left.\mathrm{CH}_{2}\right), 1.46(\mathrm{~s}, 3 \mathrm{H}$, isopropylidene $\left.\mathrm{CH}_{3}\right), 1.37\left(\mathrm{~s}, 3 \mathrm{H}\right.$, isopropylidene $\left.\mathrm{CH}_{3}\right), 1.35-1.22\left(\mathrm{~m}, 10 \mathrm{H}\right.$, octyl $\left.\mathrm{CH}_{2}\right)$, 0.89-0.84 (dd, $3 \mathrm{H}, J=7.0,7.0 \mathrm{~Hz}$, octyl $\left.\mathrm{CH}_{3}\right) ;{ }^{13} \mathrm{C} \mathrm{NMR}\left(125 \mathrm{MHz}, \mathrm{CDCl}_{3}, \delta_{\mathrm{C}}\right) 109.7$ (isopropylidene C), 101.6 (C-1), 77.3 (C-4), 75.4 (C-5), 69.9 (octyl $\mathrm{OCH}_{2}$ ), 65.4 (C-6), 54.9 (C-2), 53.9 (C-3), 31.8 (octyl $\mathrm{CH}_{2}$ ), 29.7 (octyl $\mathrm{CH}_{2}$ ), 29.3 (octyl $\mathrm{CH}_{2}$ ), 29.2 (octyl $\mathrm{CH}_{2}$ ), 26.6 (isopropylidene $\mathrm{CH}_{3}$ ), 25.9 (octyl $\mathrm{CH}_{2}$ ), 25.2 (isopropylidene $\mathrm{CH}_{3}$ ), 22.6 (octyl $\mathrm{CH}_{2}$ ), 14.1 (octyl $\left.\mathrm{CH}_{3}\right)$. HRMS (ESI) calcd. for $(\mathrm{M}+\mathrm{Na}) \mathrm{C}_{17} \mathrm{H}_{30} \mathrm{O}_{5} \mathrm{Na}: 337.1986$, found: 337.1984 .

n-Octyl 2,3-anhydro-5,6-O-isopropylidene- $\beta$-D-gulofuranoside (35). To a solution of $\mathbf{S 1}^{1}(29 \mathrm{mg}, 0.10 \mathrm{mmol})$ and 2,2-dimethoxypropane $(0.10 \mathrm{~mL}, 0.80 \mathrm{mmol})$ in acetone $(1.0 \mathrm{~mL})$ 
was added $p$-toluenesufonic acid $(0.2 \mathrm{mg})$ at $\mathrm{rt}$. The solution was stirred for $3 \mathrm{~h}$ and neutralized with $\mathrm{Et}_{3} \mathrm{~N}$. TLC showed a spot at $R_{f} 0.55\left(15: 1, \mathrm{CH}_{2} \mathrm{Cl}_{2}: \mathrm{CH}_{3} \mathrm{OH}\right)$. The mixture was concentrated and the resulting oil was dissolved in THF $(1.0 \mathrm{~mL})$, followed by the addition of $\mathrm{PPh}_{3}(31 \mathrm{mg}, 0.12 \mathrm{mmol})$. DIAD $(23 \mu \mathrm{L}, 0.12 \mathrm{mmol})$ was then added dropwise at $0{ }^{\circ} \mathrm{C}$. The reaction mixture was warmed to $\mathrm{rt}$ over $30 \mathrm{~min}$ and then concentrated. The residue was purified by chromatography (8:1, hexanes:EtOAc) to yield $\mathbf{3 5}(15 \mathrm{mg}, 49 \%$ over two steps) as a white foam. $\quad R_{f} 0.58$ (4:1, hexanes:EtOAc); $[\alpha]_{\mathrm{D}}-29.1\left(c 0.4, \mathrm{CH}_{2} \mathrm{Cl}_{2}\right) ;{ }^{1} \mathrm{H}$ NMR $(500 \mathrm{MHz}$, $\left.\mathrm{CDCl}_{3}, \delta_{\mathrm{H}}\right) 5.08(\mathrm{~s}, 1 \mathrm{H}, \mathrm{H}-1), 4.30(\mathrm{ddd}, 1 \mathrm{H}, J=7.1,6.6,6.5 \mathrm{~Hz}, \mathrm{H}-5), 4.12(\mathrm{dd}, 1 \mathrm{H}, J=8.3$, $6.5 \mathrm{~Hz}, \mathrm{H}-6), 4.04$ (d, $1 \mathrm{H}, J=7.1 \mathrm{~Hz}, \mathrm{H}-4), 3.94$ (dd, $1 \mathrm{H}, J=8.3,6.6 \mathrm{~Hz}, \mathrm{H}-6$ ), 3.79 (ddd, $1 \mathrm{H}$, $J=9.5,6.7,6.7 \mathrm{~Hz}$, octyl $\left.\mathrm{OCH}_{2}\right), 3.64(\mathrm{~d}, 1 \mathrm{H}, J=2.9 \mathrm{~Hz}, \mathrm{H}-2), 3.61(\mathrm{~d}, 1 \mathrm{H}, J=2.9 \mathrm{~Hz}, \mathrm{H}-3)$, 3.46 (ddd, $1 \mathrm{H}, J=9.5,6.7,6.7 \mathrm{~Hz}$, octyl $\left.\mathrm{OCH}_{2}\right), 1.61-1.54\left(\mathrm{~m}, 2 \mathrm{H}\right.$, octyl $\left.\mathrm{CH}_{2}\right), 1.47(\mathrm{~s}, 3 \mathrm{H}$, isopropylidene $\left.\mathrm{CH}_{3}\right), 1.38\left(\mathrm{~s}, 3 \mathrm{H}\right.$, isopropylidene $\left.\mathrm{CH}_{3}\right), 1.36-1.24\left(\mathrm{~m}, 10 \mathrm{H}\right.$, octyl $\left.\mathrm{CH}_{2}\right)$, 0.99-0.86 (dd, $3 \mathrm{H}, J=7.0,7.0 \mathrm{~Hz}$ octyl $\left.\mathrm{CH}_{3}\right) ;{ }^{13} \mathrm{C}$ NMR $\left(125 \mathrm{MHz}, \mathrm{CDCl}_{3}, \delta_{\mathrm{C}}\right) 109.8$ (isopropylidene C), 101.3 (C-1), 77.1 (C-4), 75.2 (C-5), 68.7 (octyl $\mathrm{OCH}_{2}$ ), 65.7 (C-6), 55.7 (C-2), 53.5 (C-3), 31.8 (octyl $\mathrm{CH}_{2}$ ), 29.6 (octyl $\mathrm{CH}_{2}$ ), 29.3 (octyl $\mathrm{CH}_{2}$ ), 29.2 (octyl $\mathrm{CH}_{2}$ ), 26.7 (isopropylidene $\mathrm{CH}_{3}$ ), 26.0 (octyl $\mathrm{CH}_{2}$ ), 25.2 (isopropylidene $\mathrm{CH}_{3}$ ), 22.6 (octyl $\mathrm{CH}_{2}$ ), 14.1 (octyl

$\left.\mathrm{CH}_{3}\right)$. HRMS (ESI) calcd. for $(\mathrm{M}+\mathrm{Na}) \mathrm{C}_{17} \mathrm{H}_{30} \mathrm{O}_{5} \mathrm{Na}: 337.1986$, found: 337.1984.

n-Octyl 2,3-anhydro-5,6- $O$-isopropylidene- $\alpha$-D-gulofuranosyl-(1 $\rightarrow 5)-2,3$-anhydro-

Q-D-lyxofuranoside (36). To a solution of $\mathbf{S 8}(44 \mathrm{mg}, 0.11 \mathrm{mmol})$ and 2,2-dimethoxypropane $(0.11 \mathrm{~mL}, 0.87 \mathrm{mmol})$ in acetone $(1.0 \mathrm{~mL})$ was added $p$-toluenesufonic acid $(0.2 \mathrm{mg}$, cat. $)$ at $\mathrm{rt}$. The solution was stirred for $4 \mathrm{~h}$, and neutralized with triethylamine. The mixture was 
concentrated and the resulting oil was dissolved in THF $(1.0 \mathrm{~mL})$, followed by the addition of $\mathrm{PPh}_{3}(34 \mathrm{mg}, 0.13 \mathrm{mmol})$. DIAD $(25 \mu \mathrm{L}, 0.13 \mathrm{mmol})$ was then added dropwise at $0{ }^{\circ} \mathrm{C}$. The reaction mixture was warmed to $\mathrm{rt}$ and kept strirring for $40 \mathrm{~min}$. The resulting mixture was concentrated and the residue was purified by chromatography (4:1, hexanes:EtOAc) to yield $\mathbf{3 6}$ (18 mg, 39\% over two steps) as a white foam. $\quad R_{f} 0.22\left(2: 1\right.$, hexanes:EtOAc); $[\alpha]_{\mathrm{D}}+38.3(c$ 2.3, $\left.\mathrm{CH}_{2} \mathrm{Cl}_{2}\right) ;{ }^{1} \mathrm{H}$ NMR $\left(500 \mathrm{MHz}, \mathrm{CDCl}_{3}, \delta_{\mathrm{H}}\right) 5.18(\mathrm{~s}, 1 \mathrm{H}, \mathrm{H}-1$ ') $5.02(\mathrm{~s}, 1 \mathrm{H}, \mathrm{H}-1), 4.35$ (ddd, $1 \mathrm{H}$, $J=7.3,6.7,6.6 \mathrm{~Hz}, \mathrm{H}-5$ '), $4.21(\mathrm{dd}, 1 \mathrm{H}, J=7.0,5.7 \mathrm{~Hz}, \mathrm{H}-4), 4.12(\mathrm{dd}, 1 \mathrm{H}, J=8.3,7.3 \mathrm{~Hz}$, H-6'), 4.00-3.94 (m, 2 H, H-6', H-5), 3.91 (d, 1 H, $J=6.7$ Hz, H-4'), 3.84-3.78 (m, 1 H, H-5), $3.79(\mathrm{~d}, 1 \mathrm{H}, J=2.8 \mathrm{~Hz}, \mathrm{H}-2), 3.74-3.68\left(\mathrm{~m}, 1 \mathrm{H}\right.$, octyl $\left.\mathrm{OCH}_{2}\right), 3.71$ (d, $1 \mathrm{H}, J=2.9 \mathrm{~Hz}, \mathrm{H}-2$ '), $3.64(\mathrm{~d}, 1 \mathrm{H}, J=2.8 \mathrm{~Hz}, \mathrm{H}-3), 3.60$ (d, $1 \mathrm{H}, J=2.9 \mathrm{~Hz}, \mathrm{H}-3$ '), 3.43 (ddd, $1 \mathrm{H}, J=9.3,6.9,6.9 \mathrm{~Hz}$, octyl $\left.\mathrm{OCH}_{2}\right), 1.59-1.52\left(\mathrm{~m}, 2 \mathrm{H}\right.$, octyl $\left.\mathrm{CH}_{2}\right), 1.46\left(\mathrm{~s}, 3 \mathrm{H}\right.$, isopropylidene $\left.\mathrm{CH}_{3}\right), 1.38(\mathrm{~s}, 3 \mathrm{H}$, isopropylidene $\left.\mathrm{CH}_{3}\right), 1.34-1.21$ (m, $10 \mathrm{H}$, octyl $\left.\mathrm{CH}_{2}\right), 0.89-0.85$ (m, $3 \mathrm{H}$, octyl $\left.\mathrm{CH}_{3}\right) ;{ }^{13} \mathrm{C} \mathrm{NMR}^{2}$ $\left(125 \mathrm{MHz}, \mathrm{CDCl}_{3}, \delta_{\mathrm{C}}\right) 109.82$ (isopropylidene C), 101.6 (C-1'), 101.1 (C-1), 77.4 (C-4'), 75.3 (C-5'), 74.7 (C-4), 68.5 (octyl $\mathrm{OCH}_{2}$ ), 66.9 (C-5), 65.4 (C-6'), 56.4 (C-3), 54.7 (C-2'), 54.4 (C-2), 53.9 (C-3'), 31.8 (octyl $\mathrm{CH}_{2}$ ), 29.6 (octyl $\mathrm{CH}_{2}$ ), 29.3 (octyl $\mathrm{CH}_{2}$ ), 29.2 (octyl $\mathrm{CH}_{2}$ ), 26.6 (isopropylidene $\mathrm{CH}_{3}$ ), 26.0 (octyl $\mathrm{CH}_{2}$ ), 25.2 (isopropylidene $\mathrm{CH}_{3}$ ), 22.6 (octyl $\mathrm{CH}_{2}$ ), 14.1 (octyl

$\left.\mathrm{CH}_{3}\right)$. HRMS (ESI) calcd. for $(\mathrm{M}+\mathrm{Na}) \mathrm{C}_{22} \mathrm{H}_{36} \mathrm{O}_{8} \mathrm{Na}: 451.2302$, found: 451.2308 .

$n$-Octyl 2,3-anhydro-5,6-O-isopropylidene- $\beta$-D-gulofuranosyl-( $1 \rightarrow 5)$-2,3-anhydro-

a-D-lyxofuranoside (37). To a solution of $\mathbf{S 3}(106 \mathrm{mg}, 0.26 \mathrm{mmol})$ and 2,2-dimethoxypropane $(0.26 \mathrm{~mL}, 2.1 \mathrm{mmol})$ in acetone $(2.0 \mathrm{~mL})$ was added $p$-toluenesufonic acid $(0.5 \mathrm{mg}$, cat. $)$ at $\mathrm{rt}$. The solution was stirred for $3 \mathrm{~h}$, and neutralized with triethylamine at which point TLC showed a 
spot at $R_{f} 0.58\left(10: 1, \mathrm{CH}_{2} \mathrm{Cl}_{2}: \mathrm{CH}_{3} \mathrm{OH}\right)$. The mixture was concentrated and the resulting oil was dissolved in THF $(2.0 \mathrm{~mL})$, followed by the addition of $\mathrm{PPh}_{3}(82 \mathrm{mg}, 0.31 \mathrm{mmol})$. DIAD (61 $\mu \mathrm{L}$, $0.31 \mathrm{mmol}$ ) was then added dropwise at $0{ }^{\circ} \mathrm{C}$ and then the reaction mixture was warmed to $\mathrm{rt}$ over $30 \mathrm{~min}$, and stirred for another $40 \mathrm{~min}$. The resulting mixture was concentrated and the residue was purified by chromatography (4:1, hexanes:EtOAc) to yield 37 ( $83 \mathrm{mg}, 74 \%$ over two steps) as a white foam. $R_{f} 0.27$ (4:1, hexanes:EtOAc); $[\alpha]_{\mathrm{D}}-4.0\left(c\right.$ 1.0, $\left.\mathrm{CH}_{2} \mathrm{Cl}_{2}\right) ;{ }^{1} \mathrm{H}$ NMR (500 $\left.\mathrm{MHz}, \mathrm{CDCl}_{3}, \delta_{\mathrm{H}}\right) 5.16(\mathrm{~s}, 1 \mathrm{H}, \mathrm{H}-1$ ') $, 5.05(\mathrm{~s}, 1 \mathrm{H}, \mathrm{H}-1), 4.29$ (ddd, $1 \mathrm{H}, J=6.8,6.6,6.5 \mathrm{~Hz}$, H-5'), 4.19-4.15 (m, $1 \mathrm{H}, \mathrm{H}-4), 4.10$ (dd, $1 \mathrm{H}, J=8.5,6.5 \mathrm{~Hz}, \mathrm{H}-6$ '), 4.07 (d, $1 \mathrm{H}, J=6.8 \mathrm{~Hz}$, H-4'), 3.97 (dd, 1 H, $J=10.5,5.3$ Hz, H-5), 3.94 (dd, 1 H, $J=8.5,6.6$ Hz, H-6'), 3.73 (ddd, 1 H, $J=9.6,6.7,6.7 \mathrm{~Hz}$, octyl $\mathrm{OCH}_{2}$ ), 3.72-3.68 (m, 3 H, H-2, H-3', H-5), 3.64-3.62 (m, 2 H, H-2', H-3), 3.45 (ddd, $1 \mathrm{H}, J=9.6,6.7,6.7 \mathrm{~Hz}$, octyl $\left.\mathrm{OCH}_{2}\right), 1.60-1.53\left(\mathrm{~m}, 2 \mathrm{H}\right.$, octyl $\left.\mathrm{CH}_{2}\right), 1.47$ (s, 3 $\mathrm{H}$, isopropylidene $\left.\mathrm{CH}_{3}\right), 1.38\left(\mathrm{~s}, 3 \mathrm{H}\right.$, isopropylidene $\left.\mathrm{CH}_{3}\right), 1.36-1.23\left(\mathrm{~m}, 10 \mathrm{H}\right.$, octyl $\left.\mathrm{CH}_{2}\right)$, 0.90-0.88 (m, $3 \mathrm{H}$, octyl $\left.\mathrm{CH}_{3}\right) ;{ }^{13} \mathrm{C}$ NMR (125 MHz, $\left.\mathrm{CDCl}_{3}, \delta_{\mathrm{C}}\right) 109.8$ (isopropylidene C), 101.8 (C-1'), 101.3 (C-1), 77.3 (C-4'), 75.2 (C-5'), 75.1 (C-4), 68.6 (C-5), 67.3 (octyl OCH (C), $^{2} 65.7$ (C-6'), 56.0 (C-2'), 55.6 (C-2), 54.2 (C-3'), 53.4 (C-3), 31.8 (octyl $\mathrm{CH}_{2}$ ), 29.6 (octyl $\mathrm{CH}_{2}$ ), 29.3 (octyl $\mathrm{CH}_{2}$ ), 29.2 (octyl $\mathrm{CH}_{2}$ ), 26.7 (isopropylidene $\mathrm{CH}_{3}$ ), 26.0 (octyl $\mathrm{CH}_{2}$ ), 25.2 (isopropylidene $\mathrm{CH}_{3}$ ), 22.6 (octyl $\mathrm{CH}_{2}$ ), 14.1 (octyl $\mathrm{CH}_{3}$ ). HRMS (ESI) calcd. for $\left(\mathrm{M}+\mathrm{Na}\right.$ ) $\mathrm{C}_{22} \mathrm{H}_{36} \mathrm{O}_{8} \mathrm{Na}$ : 451.2302, found: 451.2307 .

Cyclohexyl 2,3-anhydro-5,6-O-isopropylidene- $\alpha$-D-gulofuranoside (46). The glycosylation was carried out following the general protocol, and 46 was isolated by chromatography (4:1, hexanes:EtOAc) as a white foam. $R_{f} 0.25$ (4:1, hexanes: EtOAc); $[\alpha]_{\mathrm{D}}$ 
$+61.8\left(c 1.0, \mathrm{CH}_{2} \mathrm{Cl}_{2}\right) ;{ }^{1} \mathrm{H}$ NMR $\left(500 \mathrm{MHz}, \mathrm{CDCl}_{3}, \delta_{\mathrm{H}}\right) 5.19(\mathrm{~d}, 1 \mathrm{H}, J=0.6 \mathrm{~Hz}, \mathrm{H}-1), 4.36(\mathrm{ddd}$, $1 \mathrm{H}, J=6.6,6.6,6.5 \mathrm{~Hz}, \mathrm{H}-5), 4.11(\mathrm{dd}, 1 \mathrm{H}, J=8.5,6.6 \mathrm{~Hz}, \mathrm{H}-6), 4.00(\mathrm{dd}, 1 \mathrm{H}, J=8.5,6.6 \mathrm{~Hz}$, H-6), 3.87 (dd, $1 \mathrm{H}, J=6.5,0.8 \mathrm{~Hz}, \mathrm{H}-4), 3.67-3.61$ (m, $1 \mathrm{H}$, cyclohexyl OCH), 3.64 (dd, $1 \mathrm{H}, J$ $=3.0,0.6 \mathrm{~Hz}, \mathrm{H}-2), 3.58(\mathrm{dd}, 1 \mathrm{H}, J=3.0,0.8 \mathrm{~Hz}, \mathrm{H}-3), 2.01-1.89\left(\mathrm{~m}, 2 \mathrm{H}\right.$, cyclohexyl $\left.\mathrm{CH}_{2}\right)$, 1.77-1.71 (m, $2 \mathrm{H}$, cyclohexyl $\left.\mathrm{CH}_{2}\right), 1.56-1.50\left(\mathrm{~m}, 1 \mathrm{H}\right.$, cyclohexyl $\left.\mathrm{CH}_{2}\right), 1.46(\mathrm{~s}, 3 \mathrm{H}$, isopropylidene $\left.\mathrm{CH}_{3}\right), 1.37\left(\mathrm{~s}, 3 \mathrm{H}\right.$, isopropylidene $\left.\mathrm{CH}_{3}\right), 1.46-1.13\left(\mathrm{~m}, 5 \mathrm{H}\right.$, cyclohexyl $\left.\mathrm{CH}_{2}\right)$;

${ }^{13} \mathrm{C}$ NMR (125 MHz, $\left.\mathrm{CDCl}_{3}, \delta_{\mathrm{C}}\right) 109.7$ (isopropylidene C), 100.0 (C-1), 77.7 (cyclohexyl OCH), 77.3 (C-4), 75.6 (C-5), 65.5 (C-6), 55.4 (C-2), 53.8 (C-3), 33.4 (cyclohexyl $\mathrm{CH}_{2}$ ), 32.3 (cyclohexyl $\mathrm{CH}_{2}$ ), 26.6 (isopropylidene $\mathrm{CH}_{3}$ ), 25.5 (cyclohexyl $\mathrm{CH}_{2} \times 2$ ), 25.2 (isopropylidene $\mathrm{CH}_{3}$ ), 24.2 (cyclohexyl $\mathrm{CH}_{2}$ ). HRMS (ESI) calcd. for $(\mathrm{M}+\mathrm{Na}) \mathrm{C}_{15} \mathrm{H}_{24} \mathrm{O}_{5} \mathrm{Na}: 307.1516$, found: 307.1516.

tert-Butyl 2,3-anhydro-5,6-O-isopropylidene-a-D-gulofuranoside $\quad$ (47). $\quad$ The glycosylation was carried out following the general protocol, and 47 was isolated by chromatography (4:1, hexanes:EtOAc) as a white foam. $R_{f} 0.21\left(4: 1\right.$, hexanes: EtOAc); $[\alpha]_{\mathrm{D}}$ $+67.0\left(c\right.$ 1.0, $\left.\mathrm{CH}_{2} \mathrm{Cl}_{2}\right) ;{ }^{1} \mathrm{H}$ NMR $\left(400 \mathrm{MHz}, \mathrm{CDCl}_{3}, \delta_{\mathrm{H}}\right) 5.24(\mathrm{~s}, 1 \mathrm{H}, \mathrm{H}-1), 4.37(\mathrm{ddd}, 1 \mathrm{H}, J=$ 6.8, 6.5, 6.0 Hz, H-5), 4.10 (dd, $1 \mathrm{H}, J=8.5,6.5 \mathrm{~Hz}, \mathrm{H}-6), 4.05$ (dd, $1 \mathrm{H}, J=8.5,6.8 \mathrm{~Hz}, \mathrm{H}-6$ ), 3.87 (d, $1 \mathrm{H}, J=6.0 \mathrm{~Hz}, \mathrm{H}-4), 3.59-3.58(\mathrm{~m}, 2 \mathrm{H}, \mathrm{H}-2, \mathrm{H}-3), 1.47$ (s, $3 \mathrm{H}$, isopropylidene $\mathrm{CH}_{3}$ ), $1.37\left(\mathrm{~s}, 3 \mathrm{H}\right.$, isopropylidene $\left.\mathrm{CH}_{3}\right), 1.29\left(\mathrm{~s}, 9 \mathrm{H}, \mathrm{C}\left(\mathrm{CH}_{3}\right)_{3}\right) ;{ }^{13} \mathrm{C} \mathrm{NMR}\left(100 \mathrm{MHz}, \mathrm{CDCl}_{3}, \delta_{\mathrm{C}}\right)$ 109.5 (isopropylidene C), $96.5(\mathrm{C}-1), 77.0(\mathrm{C}-4), 75.6\left(\mathrm{C}\left(\mathrm{CH}_{3}\right)_{3}\right), 75.5(\mathrm{C}-5), 65.5(\mathrm{C}-6), 56.0$ (C-2), $53.5(\mathrm{C}-3), 28.6\left(\mathrm{C}\left(\mathrm{CH}_{3}\right)_{3} \times 3\right.$ ), 26.6 (isopropylidene $\mathrm{CH}_{3}$ ), 25.2 (isopropylidene $\mathrm{CH}_{3}$ ). HRMS (ESI) calcd. for $(\mathrm{M}+\mathrm{Na}) \mathrm{C}_{13} \mathrm{H}_{22} \mathrm{O}_{5} \mathrm{Na}$ : 281.1359, found: 281.1357 . 


\section{Methyl 2,3-anhydro-5,6-O-isopropylidene- $\alpha$-D-gulofuranosyl-(1 $\rightarrow$ 2)-3,5-di- $O$ -}

benzyl- $\alpha$-D-arabinofuranoside (48). The glycosylation was carried out following the general protocol, and $\mathbf{4 8}$ was isolated by chromatography (1:1, hexanes: EtOAc) as a colorless syrup. $R_{f} 0.44\left(1: 1\right.$, hexanes:EtOAc); $[\alpha]_{\mathrm{D}}+77.5\left(c\right.$ 2.0, $\left.\mathrm{CH}_{2} \mathrm{Cl}_{2}\right) ;{ }^{1} \mathrm{H}$ NMR $\left(500 \mathrm{MHz}, \mathrm{CDCl}_{3}, \delta_{\mathrm{H}}\right)$ 7.35-7.27 (m, 10 H, Ar), 5.02 (s, 1 H, H-1), 4.98 (d, 1 H, $J=0.5$ Hz, H-1'), 4.64-4.54 (m, 4 H, $\mathrm{PhCH}_{2}$ ), 4.34 (ddd, $1 \mathrm{H}, J=6.5,6.5,6.5 \mathrm{~Hz}, \mathrm{H}-5$ '), 4.25 (d, $\left.1 \mathrm{H}, J=2.9 \mathrm{~Hz}, \mathrm{H}-2\right), 4.20-4.17$ (m, $1 \mathrm{H}, \mathrm{H}-4), 4.08$ (dd, $1 \mathrm{H}, J=8.5,6.5 \mathrm{~Hz}, \mathrm{H}-6$ '), 4.01 (dd, $1 \mathrm{H}, J=8.5,6.5 \mathrm{~Hz}, \mathrm{H}-6$ '), 3.93-3.90 (m, 2 H, H-4', H-3), 3.67-3.60 (m, 3 H, H-5 × 2, H-3'), 3.54 (dd, 1 H, $J=3.0,0.5$ Hz, H-2'), $3.40\left(\mathrm{~s}, 3 \mathrm{H}, \mathrm{OCH}_{3}\right), 1.47\left(\mathrm{~s}, 3 \mathrm{H}\right.$, isopropylidene $\left.\mathrm{CH}_{3}\right), 1.34\left(\mathrm{~s}, 3 \mathrm{H}\right.$, isopropylidene $\left.\mathrm{CH}_{3}\right) ;{ }^{13} \mathrm{C}$ NMR $\left(125 \mathrm{MHz}, \mathrm{CDCl}_{3}, \delta_{\mathrm{C}}\right) 138.2(\mathrm{Ar}), 138.1(\mathrm{Ar}), 128.34(\mathrm{Ar} \times 2), 128.3(\mathrm{Ar} \times 2), 127.9(\mathrm{Ar}$ $\times$ 2), $127.8(\mathrm{Ar} \times 2), 127.7(\mathrm{Ar}), 127.6(\mathrm{Ar}), 109.7$ (isopropylidene C), $108.0(\mathrm{C}-1), 101.1\left(\mathrm{C}-1^{\prime}\right)$, 87.4 (C-2), 84.0 (C-3), 80.4 (C-4), 77.4 (C-4'), 75.1 (C-5'), $73.4\left(\mathrm{PhCH}_{2}\right), 72.4\left(\mathrm{PhCH}_{2}\right), 69.7$ (C-5), 65.4 (C-6'), $55.2\left(\mathrm{OCH}_{3}\right), 54.9$ (C-2'), 54.2 (C-3'), 26.6 (isopropylidene $\left.\mathrm{CH}_{3}\right), 25.2$ (isopropylidene $\mathrm{CH}_{3}$ ). HRMS (ESI) calcd. for $(\mathrm{M}+\mathrm{Na}) \mathrm{C}_{29} \mathrm{H}_{36} \mathrm{O}_{9} \mathrm{Na}$ : 551.2252, found: 551.2254 .

n-Octyl 2,3-anhydro-5,6-di-O-benzyl-a-D-gulofuranoside (49). The glycosylation was carried out following the general protocol, and $\mathbf{4 9}$ was isolated by chromatography $(4: 1$, hexanes:EtOAc) as a colorless oil. $\quad R_{f} 0.42\left(4: 1\right.$, hexanes: EtOAc); $[\alpha]_{\mathrm{D}}+28.9\left(c 0.4, \mathrm{CH}_{2} \mathrm{Cl}_{2}\right)$; ${ }^{1} \mathrm{H}$ NMR (500 MHz, $\left.\mathrm{CDCl}_{3}, \delta_{\mathrm{H}}\right)$ 7.40-7.27 (m, $\left.10 \mathrm{H}, \mathrm{Ar}\right), 5.08$ (d, $\left.1 \mathrm{H}, J=0.5 \mathrm{~Hz}, \mathrm{H}-1\right), 4.79$ (d, $\left.1 \mathrm{H}, J=12.0 \mathrm{~Hz}, \mathrm{PhCH}_{2}\right), 4.74\left(\mathrm{~d}, 1 \mathrm{H}, J=12.0 \mathrm{~Hz}, \mathrm{PhCH}_{2}\right), 4.60$ (d, $1 \mathrm{H}, J=12.0 \mathrm{~Hz}, \mathrm{PhCH}_{2}$ ), 4.55 (d, $1 \mathrm{H}, J=12.0 \mathrm{~Hz}, \mathrm{PhCH}_{2}$ ), 4.10 (dd, $\left.1 \mathrm{H}, J=7.1,0.8 \mathrm{~Hz}, \mathrm{H}-4\right), 3.91$ (ddd, $1 \mathrm{H}, J=7.1$, 
4.9, 3.6 Hz, H-5), 3.82-3.74 (m, 3 H, H-6 × 2, octyl $\left.\mathrm{OCH}_{2}\right), 3.67$ (dd, $\left.1 \mathrm{H}, J=3.0,0.8 \mathrm{~Hz}, \mathrm{H}-3\right)$, $3.65(\mathrm{dd}, 1 \mathrm{H}, J=3.0,0.5 \mathrm{~Hz}, \mathrm{H}-2), 3.58\left(\mathrm{ddd}, 1 \mathrm{H}, J=9.6,7.0,7.0 \mathrm{~Hz}\right.$, octyl $\left.\mathrm{OCH}_{2}\right), 1.68-1.60$ (m, $2 \mathrm{H}$, octyl $\left.\mathrm{CH}_{2}\right), 1.40-1.24\left(\mathrm{~m}, 10 \mathrm{H}\right.$, octyl $\left.\mathrm{CH}_{2}\right), 0.90-0.87$ (dd, $3 \mathrm{H}, J=7.0,7.0 \mathrm{~Hz}$, octyl $\left.\mathrm{CH}_{3}\right) ;{ }^{13} \mathrm{C} \mathrm{NMR}\left(125 \mathrm{MHz}, \mathrm{CDCl}_{3}, \delta_{\mathrm{C}}\right) 138.7(\mathrm{Ar}), 138.2(\mathrm{Ar}), 128.4(\mathrm{Ar} \times 2), 128.3(\mathrm{Ar} \times 2)$, $127.9(\mathrm{Ar} \times 2), 127.7(\mathrm{Ar} \times 2), 127.6(\mathrm{Ar}), 127.5(\mathrm{Ar}), 101.5(\mathrm{C}-1), 78.5(\mathrm{C}-5), 78.0(\mathrm{C}-4), 73.5$ $\left(\mathrm{PhCH}_{2}\right), 73.2\left(\mathrm{PhCH}_{2}\right), 70.5(\mathrm{C}-6), 69.5\left(\right.$ octyl $\left.\mathrm{OCH}_{2}\right), 55.5(\mathrm{C}-2), 54.8(\mathrm{C}-3), 31.8\left(\right.$ octyl $\left.\mathrm{CH}_{2}\right)$, 29.8 (octyl $\mathrm{CH}_{2}$ ), 29.4 (octyl $\mathrm{CH}_{2}$ ), 29.3 (octyl $\mathrm{CH}_{2}$ ), 26.0 (octyl $\mathrm{CH}_{2}$ ), 22.7 (octyl $\mathrm{CH}_{2}$ ), 14.1 (octyl $\left.\mathrm{CH}_{3}\right)$. $\quad$ HRMS (ESI) calcd. for $(\mathrm{M}+\mathrm{Na}) \mathrm{C}_{28} \mathrm{H}_{38} \mathrm{O}_{5} \mathrm{Na}$ : 477.2612, found: 477.26.13

Cyclohexyl 2,3-anhydro-5,6-di-O-benzyl-a-D-gulofuranoside $\quad$ (50). The glycosylation was carried out following the general protocol, and $\mathbf{5 0}$ was purified by chromatography (4:1, hexanes:EtOAc) as a colorless oil. $\quad R_{f} 0.34\left(4: 1\right.$, hexanes: EtOAc); $[\alpha]_{\mathrm{D}}$ -24.8 (c 0.5, $\left.\mathrm{CH}_{2} \mathrm{Cl}_{2}\right) ;{ }^{1} \mathrm{H} \mathrm{NMR}\left(500 \mathrm{MHz}, \mathrm{CDCl}_{3}, \delta_{\mathrm{H}}\right)$ 7.40-7.26 (m, $\left.10 \mathrm{H}, \mathrm{Ar}\right), 5.19(\mathrm{~s}, 1 \mathrm{H}$, H-1), 4.78 (d, $\left.1 \mathrm{H}, J=12.0 \mathrm{~Hz}, \mathrm{PhCH}_{2}\right), 4.74$ (d, $\left.1 \mathrm{H}, J=12.0 \mathrm{~Hz}, \mathrm{PhCH}_{2}\right), 4.60$ (d, $1 \mathrm{H}, J=$ 12.0 Hz, PhCH $\left.H_{2}\right), 4.55$ (d, $1 \mathrm{H}, J=12.0 \mathrm{~Hz}, \mathrm{PhCH}_{2}$ ), 4.08 (dd, $\left.1 \mathrm{H}, J=7.1,0.8 \mathrm{~Hz}, \mathrm{H}-4\right), 3.91$ (ddd, $1 \mathrm{H}, J=7.1,4.9,3.4 \mathrm{~Hz}, \mathrm{H}-5), 3.80$ (dd, $1 \mathrm{H}, J=10.8,3.4 \mathrm{~Hz}, \mathrm{H}-6), 3.77$ (dd, $1 \mathrm{H}, J=$ 10.8, 4.9 Hz, H-6), 3.65 (dd, $1 \mathrm{H}, J=2.9,0.8 \mathrm{~Hz}, \mathrm{H}-3), 3.64-3.60$ (m, $1 \mathrm{H}$, cyclohexyl OCH), $3.63(\mathrm{~d}, 1 \mathrm{H}, J=2.9 \mathrm{~Hz}, \mathrm{H}-2), 2.02-1.92\left(\mathrm{~m}, 2 \mathrm{H}\right.$, cyclohexyl $\left.\mathrm{CH}_{2}\right), 1.80-1.74(\mathrm{~m}, 2 \mathrm{H}$, cyclohexyl $\left.\mathrm{CH}_{2}\right), 1.60-1.54\left(\mathrm{~m}, 1 \mathrm{H}\right.$, cyclohexyl $\left.\mathrm{CH}_{2}\right), 1.48-1.32\left(\mathrm{~m}, 2 \mathrm{H}\right.$, cyclohexyl $\left.\mathrm{CH}_{2}\right)$, 1.30-1.15 (m, $3 \mathrm{H}$, cyclohexyl $\left.\mathrm{CH}_{2}\right) ;{ }^{13} \mathrm{C} \mathrm{NMR}\left(125 \mathrm{MHz}, \mathrm{CDCl}_{3}, \delta_{\mathrm{C}}\right) 138.8(\mathrm{Ar}), 138.3(\mathrm{Ar})$, 128.4 $(\mathrm{Ar} \times 2), 128.3(\mathrm{Ar} \times 2), 127.9(\mathrm{Ar} \times 2), 127.7(\mathrm{Ar} \times 2), 127.6(\mathrm{Ar}), 127.5(\mathrm{Ar}), 100.4$ (C-1), 78.6 (C-5), 78.1 (C-4), 78.0 (cyclohexyl OCH), $73.4\left(\mathrm{PhCH}_{2}\right), 73.2\left(\mathrm{PhCH}_{2}\right), 70.6(\mathrm{C}-6)$, 
56.0 (C-2), 54.6 (C-3), 33.6 (cyclohexyl $\mathrm{CH}_{2}$ ), 32.5 (cyclohexyl $\mathrm{CH}_{2}$ ), 25.6 (cyclohexyl $\mathrm{CH}_{2}$ ), 24.3 (cyclohexyl $\mathrm{CH}_{2} \times 2$ ). HRMS (ESI) calcd. for $(\mathrm{M}+\mathrm{Na}) \mathrm{C}_{26} \mathrm{H}_{32} \mathrm{O}_{5} \mathrm{Na}$ : 447.2141, found: 447.2142.

tert-Butyl 2,3-anhydro-5,6-di-O-benzyl-a-D-gulofuranoside (51). The glycosylation was carried out following the general protocol, and $\mathbf{5 1}$ was isolated by chromatography (4:1, hexanes:EtOAc) as a colorless oil. $R_{f} 0.22\left(4: 1\right.$, hexanes: EtOAc); $[\alpha]_{\mathrm{D}}-21.0\left(c 0.5, \mathrm{CH}_{2} \mathrm{Cl}_{2}\right)$;

${ }^{1} \mathrm{H}$ NMR $\left(500 \mathrm{MHz}, \mathrm{CDCl}_{3}, \delta_{\mathrm{H}}\right)$ 7.40-7.25 (m, $\left.10 \mathrm{H}, \mathrm{Ar}\right), 5.25$ (s, $\left.1 \mathrm{H}, \mathrm{H}-1\right), 4.79(\mathrm{~d}, 1 \mathrm{H}, J=$ $11.9 \mathrm{~Hz}, \mathrm{PhCH}_{2}$ ), 4.73 (d, $\left.1 \mathrm{H}, J=11.9 \mathrm{~Hz}, \mathrm{PhCH}_{2}\right), 4.60$ (d, $1 \mathrm{H}, J=12.0 \mathrm{~Hz}, \mathrm{PhCH}_{2}$ ), 4.56 (d, $1 \mathrm{H}, J=12.0 \mathrm{~Hz}, \mathrm{PhCH}_{2}$ ), 4.06 (dd, $\left.1 \mathrm{H}, J=7.2,0.8 \mathrm{~Hz}, \mathrm{H}-4\right), 3.92$ (ddd, $1 \mathrm{H}, J=7.2,5.0,3.5$ Hz, H-5), 3.81 (dd, 1 H, $J=10.9,3.5$ Hz, H-6), 3.77 (dd, 1 H, $J=10.9,5.0$ Hz, H-6), 3.63 (dd, 1 $\mathrm{H}, J=3.0,0.8 \mathrm{~Hz}, \mathrm{H}-3), 3.57$ (d, $1 \mathrm{H}, J=3.0 \mathrm{~Hz}, \mathrm{H}-2), 1.32\left(\mathrm{~s}, 9 \mathrm{H}, \mathrm{C}\left(\mathrm{CH}_{3}\right)_{3}\right) ;{ }^{13} \mathrm{C}$ NMR $(125$ $\left.\mathrm{MHz}, \mathrm{CDCl}_{3}, \delta_{\mathrm{C}}\right) 138.9(\mathrm{Ar}), 138.3(\mathrm{Ar}), 128.3(\mathrm{Ar} \times 2), 128.2(\mathrm{Ar} \times 2), 127.8(\mathrm{Ar} \times 2), 127.6$ $(\mathrm{Ar} \times 2), 127.55(\mathrm{Ar}), 127.4(\mathrm{Ar}), 96.3(\mathrm{C}-1), 78.8(\mathrm{C}-5), 78.2(\mathrm{C}-4), 75.6\left(C\left(\mathrm{CH}_{3}\right)_{3}\right), 73.4$ $\left(\mathrm{PhCH}_{2}\right), 73.2\left(\mathrm{PhCH}_{2}\right), 70.8(\mathrm{C}-6), 56.7(\mathrm{C}-2), 54.2(\mathrm{C}-3), 28.7\left(\mathrm{C}^{\left.\left(\mathrm{CH}_{3}\right)_{3} \times 3\right) . \quad \text { HRMS (ESI) }}\right.$ calcd. for $(\mathrm{M}+\mathrm{Na}) \mathrm{C}_{24} \mathrm{H}_{30} \mathrm{O}_{5} \mathrm{Na}: 421.1986$, found: 421.1989 .

\section{$n$-Octyl 2,3-anhydro-5,6-di- $O$-benzyl-a-D-gulofuranosyl-( $1 \rightarrow 5)$-2,3-anhydro- $\alpha$-D-}

lyxofuranoside (52). The glycosylation was carried out following the general protocol, and $\mathbf{5 2}$ was isolated by chromatgraphy (3:1, hexanes:EtOAc) as a colorless oil. $R_{f} 0.27$ (3:1, hexanes:EtOAc); $[\alpha]_{\mathrm{D}}+27.6\left(c 0.9, \mathrm{CH}_{2} \mathrm{Cl}_{2}\right) ;{ }^{1} \mathrm{H} \mathrm{NMR}\left(500 \mathrm{MHz}, \mathrm{CDCl}_{3}, \delta_{\mathrm{H}}\right) 7.40-7.25(\mathrm{~m}, 10$ H, Ar), 5.17 (s, 1 H, H-1'), 5.04 (s, 1 H, H-1), 4.77 (d, 1 H, $J=12.0$ Hz, PhCH Ph, 4.73 (d, $1 \mathrm{H}, J$ $\left.=12.0 \mathrm{~Hz}, \mathrm{PhCH}_{2}\right), 4.60\left(\mathrm{~d}, 1 \mathrm{H}, J=12.0 \mathrm{~Hz}, \mathrm{PhCH}_{2}\right), 4.55\left(\mathrm{~d}, 1 \mathrm{H}, J=12.0 \mathrm{~Hz}, \mathrm{PhCH}_{2}\right), 4.23$ 
(dd, $1 \mathrm{H}, J=7.8,5.4 \mathrm{~Hz}, \mathrm{H}-4), 4.12$ (d, $1 \mathrm{H}, J=7.0 \mathrm{~Hz}, \mathrm{H}-4$ '), 3.94 (dd, $1 \mathrm{H}, J=9.9,5.4 \mathrm{~Hz}$, H-5), 3.89 (ddd, 1 H, J= 7.0, 4.8, 3.7 Hz, H-5'), 3.82-3.72 (m, 5 H, H-5, H-3', H-6' × 2, octyl $\mathrm{OCH}_{2}$ ), 3.68 (s, $2 \mathrm{H}, \mathrm{H}-2, \mathrm{H}-3$ ), 3.66 (d, $1 \mathrm{H}, J=2.8 \mathrm{~Hz}, \mathrm{H}-2$ '), 3.45 (ddd, $1 \mathrm{H}, J=9.5,6.6,6.6$ $\mathrm{Hz}$, octyl $\left.\mathrm{OCH}_{2}\right), 1.62-1.54\left(\mathrm{~m}, 2 \mathrm{H}\right.$, octyl $\left.\mathrm{CH}_{2}\right), 1.35-1.24\left(\mathrm{~m}, 10 \mathrm{H}\right.$, octyl $\left.\mathrm{CH}_{2}\right), 0.90-0.86$ (dd, $3 \mathrm{H}, J=6.9,6.9 \mathrm{~Hz}$, octyl $\left.\mathrm{CH}_{3}\right) ;{ }^{13} \mathrm{C} \mathrm{NMR}\left(125 \mathrm{MHz}, \mathrm{CDCl}_{3}, \delta_{\mathrm{C}}\right) 138.6(\mathrm{Ar}), 138.1(\mathrm{Ar}), 128.4$ $(\mathrm{Ar} \times 2), 128.3(\mathrm{Ar} \times 2), 127.9(\mathrm{Ar} \times 2), 127.7(\mathrm{Ar} \times 2), 127.6(\mathrm{Ar}), 127.5(\mathrm{Ar}), 101.5\left(\mathrm{C}-1^{\prime}\right)$, 101.2 (C-1), 78.3 (C-5'), 78.1 (C-4), 74.6 (C-4'), $73.5\left(\mathrm{PhCH}_{2}\right), 73.1\left(\mathrm{PhCH}_{2}\right), 70.4(\mathrm{C}-6$ '), 68.6 (octyl $\mathrm{OCH}_{2}$ ), 66.8 (C-5), 56.6 (C-2'), 55.3 (C-2), 54.8 (C-3), 54.4 (C-3'), 31.8 (octyl $\mathrm{CH}_{2}$ ), 29.6 (octyl $\mathrm{CH}_{2}$ ), 29.4 (octyl $\mathrm{CH}_{2}$ ), 29.2 (octyl $\mathrm{CH}_{2}$ ), 26.0 (octyl $\mathrm{CH}_{2}$ ), 22.6 (octyl $\mathrm{CH}_{2}$ ), 14.1 (octyl $\left.\mathrm{CH}_{3}\right)$. HRMS (ESI) calcd. for $(\mathrm{M}+\mathrm{Na}) \mathrm{C}_{33} \mathrm{H}_{44} \mathrm{O}_{8} \mathrm{Na}: 591.2928$, found: 591.2926.

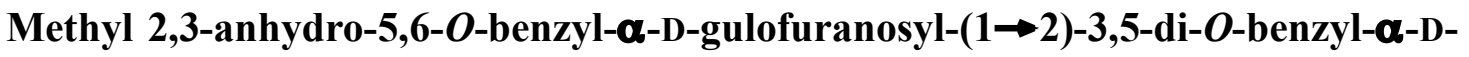

arabinofuranoside (53). The glycosylation was carried out following the general protocol, and 53 was purified by chromatography $\left(3: 1\right.$, hexanes:EtOAc) as a colorless oil. $R_{f} 0.21(3: 1$, hexanes:EtOAc); $[\alpha]_{\mathrm{D}}+53.7\left(c 0.4, \mathrm{CH}_{2} \mathrm{Cl}_{2}\right) ;{ }^{1} \mathrm{H}$ NMR $\left(500 \mathrm{MHz}, \mathrm{CDCl}_{3}, \delta_{\mathrm{H}}\right) 7.36-7.25(\mathrm{~m}, 20$ H, Ar), 5.06 (s, 1 H, H-1), 4.98 (s, $\left.1 \mathrm{H}, \mathrm{H}-1^{\prime}\right), 4.75$ (d, 1 H, $\left.J=11.7 \mathrm{~Hz}, \mathrm{PhCH}_{2}\right), 4.69$ (d, $1 \mathrm{H}, J$ $\left.=11.7 \mathrm{~Hz}, \mathrm{PhCH}_{2}\right), 4.64-4.53\left(\mathrm{~m}, 6 \mathrm{H}, \mathrm{PhCH}_{2}\right), 4.24$ (d, $\left.1 \mathrm{H}, J=2.8 \mathrm{~Hz}, \mathrm{H}-2\right), 4.20$ (ddd, $1 \mathrm{H}, J$ $=6.8,5.4,4.0 \mathrm{~Hz}, \mathrm{H}-4), 4.01(\mathrm{dd}, 1 \mathrm{H}, J=7.0,0.6 \mathrm{~Hz}, \mathrm{H}-4$ '), 3.94 (dd, $1 \mathrm{H}, J=6.8,2.8 \mathrm{~Hz}, \mathrm{H}-3)$, 3.89 (ddd, $1 \mathrm{H}, J=7.0,4.7,3.7$ Hz, H-5'), 3.80-3.74 (m, 2 H, H-6' × 2), 3.68-3.61 (m, 3 H, H-5 $\times 2, \mathrm{H}-3$ ') $, 3.52\left(\mathrm{~d}, 1 \mathrm{H}, J=3.0 \mathrm{~Hz}, \mathrm{H}-2^{\prime}\right), 3.36\left(\mathrm{~s}, 3 \mathrm{H}, \mathrm{OCH}_{3}\right) ;{ }^{13} \mathrm{C} \mathrm{NMR}\left(125 \mathrm{MHz}, \mathrm{CDCl}_{3}, \delta_{\mathrm{C}}\right)$ 138.6 (Ar), $138.2(\mathrm{Ar}), 138.1(\mathrm{Ar}), 138.0(\mathrm{Ar}), 128.5(\mathrm{Ar} \times 2), 128.4(\mathrm{Ar} \times 2), 128.33(\mathrm{Ar} \times 2)$, 128.3 $(\mathrm{Ar} \times 2), 127.9(\mathrm{Ar} \times 2), 127.8(\mathrm{Ar} \times 2), 127.7(\mathrm{Ar} \times 3), 127.69(\mathrm{Ar} \times 2), 127.65(\mathrm{Ar})$, 
127.6 (Ar), 127.55 (Ar), 107.8 (C-1), 101.4 (C-1'), 88.1 (C-2), 83.9 (C-3), 80.4 (C-4), 78.7

(C-5'), 78.3 (C-4'), $73.5\left(\mathrm{PhCH}_{2}\right), 73.4\left(\mathrm{PhCH}_{2}\right), 73.3\left(\mathrm{PhCH}_{2}\right), 72.4\left(\mathrm{PhCH}_{2}\right), 70.5(\mathrm{C}-6$ '), 69.8

(C-5), 55.3 (C-2'), $55.1\left(\mathrm{OCH}_{3}\right), 54.7$ (C-3'). HRMS (ESI) calcd. for $(\mathrm{M}+\mathrm{Na}) \mathrm{C}_{40} \mathrm{H}_{44} \mathrm{O}_{9} \mathrm{Na}$ : 691.2878, found: 691.2878 .

n-Octyl 2,3-anhydro-5,6-di- $\boldsymbol{O}$-benzoyl- $\boldsymbol{\alpha}$-D-gulofuranoside (54). The glycosylation was carried out following the general protocol, and $\mathbf{5 4}$ was isolated by chromatography (4:1, hexanes:EtOAc) as a white foam. $\quad R_{f} 0.26$ (4:1, hexanes: EtOAc); $[\alpha]_{\mathrm{D}}+44.1\left(c 0.3, \mathrm{CH}_{2} \mathrm{Cl}_{2}\right)$; ${ }^{1} \mathrm{H}$ NMR (500 MHz, $\left.\mathrm{CDCl}_{3}, \delta_{\mathrm{H}}\right)$ 8.10-8.07 (m, $\left.2 \mathrm{H}, \mathrm{Ar}\right), 8.03$ (m, $\left.2 \mathrm{H}, \mathrm{Ar}\right), 7.59-7.50(\mathrm{~m}, 2 \mathrm{H}$, Ar), 7.46-7.39 (m, 4 H, Ar), 5.77 (dd, $1 \mathrm{H}, J=10.1,5.5 \mathrm{~Hz}, \mathrm{H}-5), 5.13$ (d, $1 \mathrm{H}, J=0.5 \mathrm{~Hz}, \mathrm{H}-1)$, 4.82-4.78 (m, $2 \mathrm{H}, \mathrm{H}-6 \times$ 2), $4.24(\mathrm{dd}, 1 \mathrm{H}, J=5.5,0.8 \mathrm{~Hz}, \mathrm{H}-4), 3.82(\mathrm{dd}, 1 \mathrm{H}, J=3.0,0.8 \mathrm{~Hz}$, H-3), 3.77 (ddd, $1 \mathrm{H}, J=9.6,6.6,6.6 \mathrm{~Hz}$, octyl $\mathrm{OCH}_{2}$ ), 3.71 (dd, $\left.1 \mathrm{H}, J=3.0,0.5 \mathrm{~Hz}, \mathrm{H}-2\right), 3.62$ (ddd, $1 \mathrm{H}, J=9.6,6.9,6.9 \mathrm{~Hz}$, octyl $\left.\mathrm{OCH}_{2}\right), 1.65-1.59\left(\mathrm{~m}, 2 \mathrm{H}\right.$, octyl $\left.\mathrm{CH}_{2}\right), 1.38-1.22(\mathrm{~m}, 10 \mathrm{H}$, octyl $\left.\mathrm{CH}_{2}\right), 0.87\left(\mathrm{dd}, 3 \mathrm{H}, J=7.0,7.0 \mathrm{~Hz}\right.$, octyl $\left.\mathrm{CH}_{3}\right) ;{ }^{13} \mathrm{C} \mathrm{NMR}\left(125 \mathrm{MHz}, \mathrm{CDCl}_{3}, \delta_{\mathrm{C}}\right) 166.1$ $(\mathrm{C}=\mathrm{O}), 165.8(\mathrm{C}=\mathrm{O}), 133.2(\mathrm{Ar}), 133.1(\mathrm{Ar}), 129.9(\mathrm{Ar} \times 2), 129.84(\mathrm{Ar}), 129.8(\mathrm{Ar}), 129.7(\mathrm{Ar}$ $\times 2), 128.4(\mathrm{Ar} \times 4), 101.2(\mathrm{C}-1), 74.8(\mathrm{C}-4), 71.6(\mathrm{C}-5), 69.3\left(\right.$ octyl $\left.\mathrm{OCH}_{2}\right), 63.4(\mathrm{C}-6), 55.4$ (C-2), 54.1 (C-3), 31.8 (octyl $\mathrm{CH}_{2}$ ), 29.8 (octyl $\mathrm{CH}_{2}$ ), 29.4 (octyl $\mathrm{CH}_{2}$ ), 29.2 (octyl $\mathrm{CH}_{2}$ ), 26.0 (octyl $\mathrm{CH}_{2}$ ), 22.6 (octyl $\mathrm{CH}_{2}$ ), 14.1 (octyl $\mathrm{CH}_{3}$ ). $\quad$ HRMS (ESI) calcd. for $\left(\mathrm{M}+\mathrm{Na}\right.$ ) $\mathrm{C}_{28} \mathrm{H}_{34} \mathrm{O}_{7} \mathrm{Na}$ : 505.2197, found: 505.2197.

Cyclohexyl 2,3-anhydro-5,6-di- $\boldsymbol{O}$-benzoyl-a-D-gulofuranoside (55). The glycosylation was carried out following the general protocol, and $\mathbf{5 5}$ was isolated by chromatography (4:1, hexanes:EtOAc) as a white foam. $R_{f} 0.19$ (4:1, hexanes: EtOAc); $[\alpha]_{\mathrm{D}}$ 
$+67.9\left(c 0.7, \mathrm{CH}_{2} \mathrm{Cl}_{2}\right) ;{ }^{1} \mathrm{H}$ NMR $\left(500 \mathrm{MHz}, \mathrm{CDCl}_{3}, \delta_{\mathrm{H}}\right) 8.10-8.07(\mathrm{~m}, 2 \mathrm{H}, \mathrm{Ar}), 8.03-8.00(\mathrm{~m}, 2$ H, Ar), 7.58-7.51 (m, 2 H, Ar), 7.46-7.39 (m, 4 H, Ar), 5.78 (ddd, 1 H, J= 5.4, 5.0, 5.0 Hz, H-5), $5.22(\mathrm{~d}, 1 \mathrm{H}, J=0.5 \mathrm{~Hz}, \mathrm{H}-1), 4.80(\mathrm{~d}, 2 \mathrm{H}, J=5.0 \mathrm{~Hz}, \mathrm{H}-6 \times 2), 4.22(\mathrm{dd}, 1 \mathrm{H}, J=5.4,0.9 \mathrm{~Hz}$, H-4), 3.80 (dd, $1 \mathrm{H}, J=2.9,0.9 \mathrm{~Hz}, \mathrm{H}-3), 3.70$ (dd, $1 \mathrm{H}, J=2.9,0.5 \mathrm{~Hz}, \mathrm{H}-2), 3.69-3.60$ (m, $1 \mathrm{H}$, cyclohexyl OCH), 1.99-1.87 (m, $2 \mathrm{H}$, cyclohexyl $\mathrm{CH}_{2}$ ), 1.78-1.70 (m, $2 \mathrm{H}$, cyclohexyl $\mathrm{CH}_{2}$ ), 1.57-1.48 (m, $1 \mathrm{H}$, cyclohexyl $\left.\mathrm{CH}_{2}\right), 1.45-1.15\left(\mathrm{~m}, 5 \mathrm{H}\right.$, cyclohexyl $\left.\mathrm{CH}_{2}\right) ;{ }^{13} \mathrm{C}$ NMR $(125 \mathrm{MHz}$, $\left.\mathrm{CDCl}_{3}, \delta_{\mathrm{C}}\right) 166.1(\mathrm{C}=\mathrm{O}), 165.8(\mathrm{C}=\mathrm{O}), 133.2(\mathrm{Ar}), 133.1(\mathrm{Ar}), 129.9(\mathrm{Ar} \times 2), 129.8(\mathrm{Ar} \times 2)$, 129.7 $(\mathrm{Ar} \times 2), 129.4(\mathrm{Ar} \times 2), 128.4(\mathrm{Ar} \times 2), 100.2(\mathrm{C}-1), 77.6($ cyclohexyl OCH $), 74.8(\mathrm{C}-4)$, 71.7 (C-5), 63.5 (C-6), 56.0 (C-2), 54.2 (C-3), 33.5 (cyclohexyl $\mathrm{CH}_{2}$ ), 32.6 (cyclohexyl $\mathrm{CH}_{2}$ ), 25.5 (cyclohexyl $\left.\mathrm{CH}_{2}\right), 24.2\left(\right.$ cyclohexyl $\left.\mathrm{CH}_{2}\right), 24.1\left(\right.$ cyclohexyl $\left.\mathrm{CH}_{2}\right)$. HRMS (ESI) calcd. for $(\mathrm{M}+\mathrm{Na}) \mathrm{C}_{26} \mathrm{H}_{28} \mathrm{O}_{7} \mathrm{Na}: 475.1727$, found: 475.1730 .

tert-Butyl 2,3-anhydro-5,6-di-O-benzoyl-a-D-gulofuranoside (56). The glycosylation was carried out following the general protocol, and $\mathbf{5 6}$ was isolated by chromatography (4:1, hexanes:EtOAc) as a white foam. $R_{f} 0.17$ (4:1, hexanes: EtOAc); $[\alpha]_{\mathrm{D}}+82.5\left(c 0.4, \mathrm{CH}_{2} \mathrm{Cl}_{2}\right) ;{ }^{1} \mathrm{H}$ NMR (500 MHz, $\left.\mathrm{CDCl}_{3}, \delta_{\mathrm{H}}\right)$ 8.10-8.07 (m, $\left.2 \mathrm{H}, \mathrm{Ar}\right), 8.03-8.00(\mathrm{~m}, 2 \mathrm{H}, \mathrm{Ar}), 7.58-7.51(\mathrm{~m}, 2 \mathrm{H}$, Ar), 7.46-7.38 (m, 4 H, Ar), 5.77 (ddd, $1 \mathrm{H}, J=5.9,5.6,4.5 \mathrm{~Hz}, \mathrm{H}-5), 5.29$ (d, $1 \mathrm{H}, J=0.6 \mathrm{~Hz}$, H-1), 4.84-4.80 (m, 2 H, H-6 × 2), 4.21 (dd, 1 H, $J=5.6,0.9$ Hz, H-4), 3.79 (dd, 1 H, $J=2.9$, $0.9 \mathrm{~Hz}, \mathrm{H}-3), 3.65(\mathrm{dd}, 1 \mathrm{H}, J=2.9,0.6 \mathrm{~Hz}, \mathrm{H}-2), 1.27\left(\mathrm{~s}, 9 \mathrm{H}, \mathrm{C}\left(\mathrm{CH}_{3}\right)_{3} \times 3\right) ;{ }^{13} \mathrm{C} \mathrm{NMR}(125$ $\left.\mathrm{MHz}, \mathrm{CDCl}_{3}, \delta_{\mathrm{C}}\right) 166.1(\mathrm{C}=\mathrm{O}), 165.8(\mathrm{C}=\mathrm{O}), 133.1(\mathrm{Ar}), 133.0(\mathrm{Ar}), 129.93(\mathrm{Ar}), 129.9(\mathrm{Ar})$, $129.8(\mathrm{Ar} \times 2), 129.7(\mathrm{Ar} \times 2), 128.4(\mathrm{Ar} \times 2), 128.35(\mathrm{Ar} \times 2), 96.4(\mathrm{C}-1), 75.8\left(C\left(\mathrm{CH}_{3}\right)_{3}\right), 75.0$ 
(C-4), 71.9 (C-5), 63.5 (C-6), 56.9 (C-2), 53.7 (C-3), $\left.28.6\left(\mathrm{C}_{(\mathrm{CH}}\right)_{3} \times 3\right) . \quad$ HRMS (ESI) calcd. for $(\mathrm{M}+\mathrm{Na}) \mathrm{C}_{24} \mathrm{H}_{26} \mathrm{O}_{7} \mathrm{Na}: 449.1571$, found: 449.1573 .

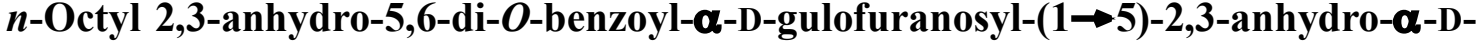

lyxofuranoside (57). The glycosylation was carried out following the general protocol, and $\mathbf{5 7}$ was isolated by chromatography $\left(2: 1\right.$, hexanes:EtOAc) as a white foam. $R_{f} 0.18(2: 1$, hexanes:EtOAc); $[\alpha]_{\mathrm{D}}+42.6\left(c 0.6, \mathrm{CH}_{2} \mathrm{Cl}_{2}\right) ;{ }^{1} \mathrm{H} \mathrm{NMR}\left(500 \mathrm{MHz}, \mathrm{CDCl}_{3}, \delta_{\mathrm{H}}\right) 8.10-8.07(\mathrm{~m}, 2 \mathrm{H}$, Ar), 8.03-8.00 (m, 2 H, Ar), 7.58-7.52 (m, 2 H, Ar), 7.46-7.39 (m, 4 H, Ar), 5.78 (ddd, 1 H, J= 5.5, 5.1, 5.1 Hz, H-5'), 5.23 (d, 1 H, $J=0.5$ Hz, H-1'), 5.03 (s, 1 H, H-1), 4.78 (d, 2 H, J= 5.1 Hz, H-6' × 2), 4.27 (dd, $1 \mathrm{H}, J=5.5,0.9 \mathrm{~Hz}, \mathrm{H}-4$ '), 4.22 (dd, $1 \mathrm{H}, J=6.9,6.0 \mathrm{~Hz}, \mathrm{H}-4)$, 3.94-3.87 (m, 2 H, H-5 × 2), $3.84(\mathrm{dd}, 1 \mathrm{H}, J=2.9,0.9 \mathrm{~Hz}, \mathrm{H}-3$ '), $3.82(\mathrm{dd}, 1 \mathrm{H}, J=2.9,0.5 \mathrm{~Hz}$, H-2'), 3.75 (d, $1 \mathrm{H}, J=2.8 \mathrm{~Hz}, \mathrm{H}-3), 3.72\left(\mathrm{ddd}, 1 \mathrm{H}, J=9.5,6.7,6.7 \mathrm{~Hz}\right.$, octyl $\left.\mathrm{OCH}_{2}\right), 3.64$ (d, 1 $\mathrm{H}, J=2.8 \mathrm{~Hz}, \mathrm{H}-2), 3.43\left(\mathrm{ddd}, 1 \mathrm{H}, J=9.5,6.8,6.8\right.$, octyl $\left.\mathrm{OCH}_{2}\right), 1.58-1.52$ (m, $2 \mathrm{H}$, octyl $\left.\mathrm{CH}_{2}\right), 1.35-1.23\left(\mathrm{~m}, 10 \mathrm{H}\right.$, octyl $\left.\mathrm{CH}_{2}\right), 0.88-0.86\left(\mathrm{~m}, 3 \mathrm{H}\right.$, octyl $\left.\mathrm{CH}_{3}\right) ;{ }^{13} \mathrm{C} \mathrm{NMR}(125 \mathrm{MHz}$, $\left.\mathrm{CDCl}_{3}, \delta_{\mathrm{C}}\right) 166.1(\mathrm{C}=\mathrm{O}), 165.8(\mathrm{C}=\mathrm{O}), 133.2(\mathrm{Ar}), 133.1(\mathrm{Ar}), 129.9(\mathrm{Ar} \times 2), 129.8(\mathrm{Ar} \times 2)$, 129.7 $(\mathrm{Ar} \times 2), 128.4(\mathrm{Ar} \times 4), 101.3\left(\mathrm{C}-1^{\prime}\right), 101.2(\mathrm{C}-1), 75.0\left(\mathrm{C}-4^{\prime}\right), 74.8(\mathrm{C}-4), 71.5\left(\mathrm{C}-5^{\prime}\right)$, 68.5 (octyl $\mathrm{OCH}_{2}$ ), 66.7 (C-5), 63.3 (C-6’), 56.5 (C-2), 55.3 (C-3), 54.4 (C-2’), 54.2 (C-3'), 31.8 (octyl $\mathrm{CH}_{2}$ ), 29.6 (octyl $\mathrm{CH}_{2}$ ), 29.3 (octyl $\mathrm{CH}_{2}$ ), 29.2 (octyl $\mathrm{CH}_{2}$ ), 26.0 (octyl $\mathrm{CH}_{2}$ ), 22.6 (octyl $\mathrm{CH}_{2}$ ), 14.1 (octyl $\mathrm{CH}_{3}$ ). HRMS (ESI) calcd. for $(\mathrm{M}+\mathrm{Na}) \mathrm{C}_{33} \mathrm{H}_{40} \mathrm{O}_{10} \mathrm{Na}$ : 619.2514, found: 619.2518

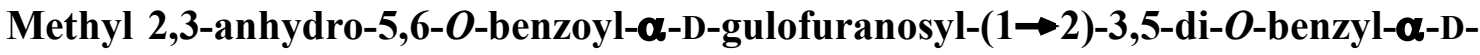

arabinofuranoside (58). The glycosylation was carried out following the general protocol, and 
58 was isolated by chromatography (2:1, hexanes:EtOAc) as a colorless syrup. $R_{f} 0.26(2: 1$, hexanes:EtOAc); $[\alpha]_{\mathrm{D}}+59.3\left(c 0.6, \mathrm{CH}_{2} \mathrm{Cl}_{2}\right) ;{ }^{1} \mathrm{H} \mathrm{NMR}\left(500 \mathrm{MHz}, \mathrm{CDCl}_{3}, \delta_{\mathrm{H}}\right) 8.09-8.06(\mathrm{~m}, 2 \mathrm{H}$, Ar), 8.04-8.00 (m, 2 H, Ar), 7.58-7.53 (m, 2 H, Ar), 7.45-7.40 (m, 4 H, Ar), 7.35-7.24 (m, 10 H, Ar), 5.78 (ddd, 1 H, J = 5.7, 5.0, 5.0 Hz, H-5'), 5.03 (s, 1 H, H-1), 5.00 (s, 1 H, H-1'), 4.76 (d, 2 H, $\left.J=5.0 \mathrm{~Hz}, \mathrm{H}-6^{\prime} \times 2\right), 4.64-4.56\left(\mathrm{~m}, 4 \mathrm{H}, \mathrm{PhCH}_{2}\right), 4.27$ (d, $1 \mathrm{H}, J=5.7 \mathrm{~Hz}, \mathrm{H}-4$ ') 4.22 (d, 1 H, $J=3.0 \mathrm{~Hz}, \mathrm{H}-2), 4.20-4.16$ (m, $1 \mathrm{H}, \mathrm{H}-4), 3.93$ (dd, $1 \mathrm{H}, J=6.9,3.0 \mathrm{~Hz}, \mathrm{H}-3), 3.82(\mathrm{~d}, 1 \mathrm{H}, J$ $=2.9 \mathrm{~Hz}, \mathrm{H}-3$ '), 3.67 (dd, $1 \mathrm{H}, J=10.8,3.7 \mathrm{~Hz}, \mathrm{H}-5), 3.64$ (dd, $1 \mathrm{H}, J=10.8,5.4 \mathrm{~Hz}, \mathrm{H}-5), 3.61$ (d, $1 \mathrm{H}, J=2.9 \mathrm{~Hz}, \mathrm{H}-2$ ') $3.28\left(\mathrm{~s}, 3 \mathrm{H}, \mathrm{OCH}_{3}\right) ;{ }^{13} \mathrm{C} \mathrm{NMR}\left(125 \mathrm{MHz}, \mathrm{CDCl}_{3}, \delta_{\mathrm{C}}\right) 166.0(\mathrm{C}=\mathrm{O})$, $165.7(\mathrm{C}=\mathrm{O}), 138.2(\mathrm{Ar}), 138.1(\mathrm{Ar}), 133.22(\mathrm{Ar}), 133.2(\mathrm{Ar}), 129.9(\mathrm{Ar} \times 2), 129.8(\mathrm{Ar} \times 2)$, 129.7 (Ar), 129.66 (Ar), 128.44 ( $\mathrm{Ar} \times 2), 128.4(\mathrm{Ar} \times 2), 128.34(\mathrm{Ar} \times 2), 128.3(\mathrm{Ar} \times 2), 127.9$ $(\mathrm{Ar} \times 2), 127.8(\mathrm{Ar} \times 2), 127.7(\mathrm{Ar}), 127.6(\mathrm{Ar}), 107.8(\mathrm{C}-1), 101.4(\mathrm{C}-1$ ') $, 88.2(\mathrm{C}-2), 84.2$ (C-3), 80.1 (C-4), 75.4 (C-4'), $73.4\left(\mathrm{PhCH}_{2}\right), 72.5\left(\mathrm{PhCH}_{2}\right), 71.5$ (C-5'), 69.8 (C-5), 63.3 (C-6'), $55.5\left(\mathrm{C}-2^{\prime}\right), 55.0\left(\mathrm{OCH}_{3}\right), 54.3\left(\mathrm{C}-3^{\prime}\right)$. HRMS (ESI) calcd. for $(\mathrm{M}+\mathrm{Na}) \mathrm{C}_{40} \mathrm{H}_{40} \mathrm{O}_{11} \mathrm{Na}$ : 719.2463, found: 719.2461 .

$n$-Octyl 3-O-benzyl-5,6- $O$-isopropylidene- $\alpha$-D-galactofuranoside (64) and $n$-Octyl 2-O-benzyl-5,6-O-isopropylidene-a-D-idofuranoside (65). Compound 34 was subjected to the general epoxide opening procedure, yielding seperable 64 and 65 as colorless oils. 64: $R_{f}$ 0.46 (4:1, hexanes:EtOAc); $[\alpha]_{\mathrm{D}}+36.5\left(c \quad 0.2, \mathrm{CH}_{2} \mathrm{Cl}_{2}\right) ;{ }^{1} \mathrm{H}$ NMR $\left(500 \mathrm{MHz}, \mathrm{CDCl}_{3}, \delta_{\mathrm{H}}\right)$ 7.40-7.27 (m, 5 H, Ar), 4.98 (d, $1 \mathrm{H}, J=4.8 \mathrm{~Hz}, \mathrm{H}-1), 4.87$ (d, $1 \mathrm{H}, J=11.7 \mathrm{~Hz}, \mathrm{PhCH}_{2}$ ), 4.62 (d, $1 \mathrm{H}, J=11.7 \mathrm{~Hz}, \mathrm{PhCH}_{2}$ ), 4.27 (ddd, $\left.1 \mathrm{H}, J=9.8,6.2,4.8 \mathrm{~Hz}, \mathrm{H}-2\right), 4.11$ (dd, $1 \mathrm{H}, J=7.3$, $7.3 \mathrm{~Hz}, \mathrm{H}-4), 3.91-3.84$ (m, $3 \mathrm{H}, \mathrm{H}-5, \mathrm{H}-6$, octyl $\mathrm{OCH}_{2}$ ), 3.80-3.74 (m, $\left.2 \mathrm{H}, \mathrm{H}-3, \mathrm{H}-6\right), 3.48$ 
(ddd, $1 \mathrm{H}, J=9.6,6.7,6.7 \mathrm{~Hz}$, octyl $\left.\mathrm{OCH}_{2}\right), 2.67(\mathrm{~d}, 1 \mathrm{H}, J=9.8 \mathrm{~Hz}, \mathrm{OH}), 1.64-1.54(\mathrm{~m}, 2 \mathrm{H}$, octyl $\left.\mathrm{CH}_{2}\right), 1.43\left(\mathrm{~s}, 3 \mathrm{H}\right.$, isopropylidene $\left.\mathrm{CH}_{3}\right), 1.37\left(\mathrm{~s}, 3 \mathrm{H}\right.$, isopropylidene $\left.\mathrm{CH}_{3}\right), 1.38-1.24(\mathrm{~m}$, $10 \mathrm{H}$, octyl $\left.\mathrm{CH}_{2}\right), 0.92-0.87\left(\mathrm{~m}, 3 \mathrm{H}\right.$, octyl $\left.\mathrm{CH}_{3}\right) ;{ }^{13} \mathrm{C} \mathrm{NMR}\left(125 \mathrm{MHz}, \mathrm{CDCl}_{3}, \delta_{\mathrm{C}}\right) 137.6(\mathrm{Ar})$, $128.4(\mathrm{Ar} \times 2), 128.0(\mathrm{Ar}), 127.9(\mathrm{Ar} \times 2), 109.7$ (isopropylidene $\mathrm{C}), 101.6(\mathrm{C}-1), 83.8(\mathrm{C}-3)$, 81.9 (C-5), 78.2 (C-4), 78.0 (C-2), $71.7\left(\mathrm{PhCH}_{2}\right), 68.8$ (octyl $\left.\mathrm{OCH}_{2}\right), 65.2$ (C-6), 31.8 (octyl $\mathrm{CH}_{2}$ ), 29.5 (octyl $\mathrm{CH}_{2}$ ), 29.3 (octyl $\mathrm{CH}_{2}$ ), 29.2 (octyl $\mathrm{CH}_{2}$ ), 26.6 (isopropylidene $\mathrm{CH}_{3}$ ), 26.1 (octyl $\mathrm{CH}_{2}$ ), 25.5 (isopropylidene $\mathrm{CH}_{3}$ ), 22.6 (octyl $\mathrm{CH}_{2}$ ), 14.1 (octyl $\mathrm{CH}_{3}$ ). HRMS (ESI) calcd. for $(\mathrm{M}+\mathrm{Na}) \mathrm{C}_{24} \mathrm{H}_{38} \mathrm{O}_{6} \mathrm{Na}: 445.2561$, found: 445.2559. 65: $R_{f} 0.38$ (4:1, hexanes:EtOAc); $[\alpha]_{\mathrm{D}}$ $+35.6\left(c\right.$ 0.26, $\left.\mathrm{CH}_{2} \mathrm{Cl}_{2}\right) ;{ }^{1} \mathrm{H}$ NMR $\left(500 \mathrm{MHz}, \mathrm{CDCl}_{3}, \delta_{\mathrm{H}}\right) 7.40-7.29(\mathrm{~m}, 5 \mathrm{H}, \mathrm{Ar}), 5.09(\mathrm{~s}, 1 \mathrm{H}$, H-1), 4.61 (s, 2 H, $\mathrm{PhCH}_{2}$ ), 4.32 (ddd, $1 \mathrm{H}, J=7.3,7.2,7.2 \mathrm{~Hz}, \mathrm{H}-5$ ), 4.24-4.19 (m, $2 \mathrm{H}, \mathrm{H}-4$, H-6), 4.08 (br s, $1 \mathrm{H}, \mathrm{H}-3$ ), 3.94 (s, $1 \mathrm{H}, \mathrm{H}-2$ ), 3.85 (ddd, $1 \mathrm{H}, J=9.6,6.8,6.8 \mathrm{~Hz}$, octyl $\mathrm{OCH}_{2}$ ), $3.73(\mathrm{dd}, 1 \mathrm{H}, J=8.3,7.2 \mathrm{~Hz}, \mathrm{H}-6), 3.42$ (ddd, $1 \mathrm{H}, J=9.6,6.6,6.6 \mathrm{~Hz}$, octyl $\left.\mathrm{OCH}_{2}\right), 2.99$ (d, 1 $\mathrm{H}, J=11.3 \mathrm{~Hz}, \mathrm{OH}), 1.60-1.52\left(\mathrm{~m}, 2 \mathrm{H}\right.$, octyl $\left.\mathrm{CH}_{2}\right), 1.52\left(\mathrm{~s}, 3 \mathrm{H}\right.$, isopropylidene $\left.\mathrm{CH}_{3}\right), 1.45(\mathrm{~s}, 3$ $\mathrm{H}$, isopropylidene $\left.\mathrm{CH}_{3}\right), 1.35-1.24\left(\mathrm{~m}, 10 \mathrm{H}\right.$, octyl $\left.\mathrm{CH}_{2}\right), 0.92-0.85\left(\mathrm{~m}, 3 \mathrm{H}\right.$, octyl $\left.\mathrm{CH}_{3}\right) ;{ }^{13} \mathrm{C}$ NMR $\left(125 \mathrm{MHz}, \mathrm{CDCl}_{3}, \delta_{\mathrm{C}}\right) 137.3(\mathrm{Ar}), 128.5(\mathrm{Ar} \times 2), 128.0(\mathrm{Ar}), 127.7(\mathrm{Ar} \times 2), 109.7$ (isopropylidene C), 105.6 (C-1), 86.2 (C-2), 84.9 (C-4), 77.0 (C-5), $74.4(\mathrm{C}-3), 72.0\left(\mathrm{PhCH}_{2}\right)$, 68.2 (octyl $\mathrm{OCH}_{2}$ ), 66.1 (C-6), 31.8 (octyl $\mathrm{CH}_{2}$ ), 29.5 (octyl $\mathrm{CH}_{2}$ ), 29.3 (octyl $\mathrm{CH}_{2}$ ), 29.2 (octyl $\mathrm{CH}_{2}$ ), 26.7 (isopropylidene $\mathrm{CH}_{3}$ ), 26.1 (octyl $\mathrm{CH}_{2}$ ), 25.6 (isopropylidene $\mathrm{CH}_{3}$ ), 22.6 (octyl $\mathrm{CH}_{2}$ ), 14.1 (octyl $\left.\mathrm{CH}_{3}\right)$. $\quad$ HRMS (ESI) calcd. for $(\mathrm{M}+\mathrm{Na}) \mathrm{C}_{24} \mathrm{H}_{38} \mathrm{O}_{6} \mathrm{Na}$ : 445.2561 , found: 445.2562 .

$n$-Octyl 3,5,6-tri- $O$-benzyl- $\alpha$-D-galactofuranoside $\quad(66) \quad$ and $n$-Octyl 2,5,6-tri- $\boldsymbol{O}$-benzyl- $\boldsymbol{\alpha}$-D-idofuranoside (67). Compound 49 was subjected to the general S29 
epoxide opening procedure, yielding an inseparable mixture of 66 and 67 as colorless oils: $R_{f}$ 0.41 (4:1, hexanes:EtOAc); ${ }^{1} \mathrm{H}$ NMR $\left(500 \mathrm{MHz}, \mathrm{CDCl}_{3}, \delta_{\mathrm{H}}\right) 7.43-7.25(\mathrm{~m}, 15 \mathrm{H}, \mathrm{Ar}), 5.08(\mathrm{~s}$, $0.74 \mathrm{H}$, Ido H-1), 4.96 (d, 0.26 H, $J=4.7 \mathrm{~Hz}, \mathrm{Gal} \mathrm{H}-1), 4.82-4.74\left(\mathrm{~m}, 2 \mathrm{H}, \mathrm{PhCH}_{2}\right), 4.63-4.52$ (m, $4 \mathrm{H}, \mathrm{PhCH}_{2}$ ), 4.40 (dd, 0.74 H, J= 7.4, 3.8 Hz, Ido H-4), 4.24 (br s, 0.26 H, Gal H-2), 4.12 (br s, 0.74 H, Ido H-3), 4.05-3.99 (m, 0.52 H, Gal H-3, Gal H-4), 3.74 (s, 0.74 H, Ido H-2), 3.71 (ddd, 0.74 H, $J=7.4,4.6,4.6$ Hz, Ido H-5), 3.80-3.64 (m, 3.26 H, Gal H-5, Gal H-6 × 2, Ido H-6 $\times 2$, octyl $\left.\mathrm{OCH}_{2}\right), 3.45-3.40\left(\mathrm{~m}, 0.26 \mathrm{H}, \mathrm{Gal}\right.$ octyl $\left.\mathrm{OCH}_{2}\right), 3.39(\mathrm{ddd}, 0.74 \mathrm{H}, J=9.5,6.6,6.6 \mathrm{~Hz}$, Ido octyl $\left.\mathrm{OCH}_{2}\right), 3.27$ (d, $0.74 \mathrm{H}, J=9.2 \mathrm{~Hz}$, Ido OH), $2.71(\mathrm{~d}, 0.26 \mathrm{H}, J=7.0 \mathrm{~Hz}, \mathrm{Gal} \mathrm{OH})$, 1.58-1.47 (m, $2 \mathrm{H}$, octyl $\left.\mathrm{CH}_{2}\right), 1.34-1.25\left(\mathrm{~m}, 10 \mathrm{H}\right.$, octyl $\left.\mathrm{CH}_{2}\right), 0.92-0.89\left(\mathrm{~m}, 3 \mathrm{H}\right.$, octyl $\left.\mathrm{CH}_{3}\right)$;

${ }^{13} \mathrm{C}$ NMR (125 MHz, $\left.\mathrm{CDCl}_{3}, \delta_{\mathrm{C}}\right) 138.7$ (Ar), 138.3 (Ar), 138.1 (Ar), 138.0 (Ar), 137.5 (Ar), 128.5 (Ar), $128.4(\mathrm{Ar}), 128.35$ (Ar), $128.3(\mathrm{Ar}), 128.2(\mathrm{Ar}), 128.0(\mathrm{Ar}), 127.9(\mathrm{Ar}), 127.86(\mathrm{Ar})$ 127.8 (Ar), 127.7 (Ar), 127.68 (Ar), 127.6 (Ar), 127.57 (Ar), 127.5 (Ar), 127.46 (Ar), 105.6 (Ido C-1), 101.2 (Gal C-1), 86.7 (Ido C-2), 83.9 (Ido C-4), 83.3 (Gal C-3), 81.3 (Gal C-4), 79.0 (Gal C-5), 78.5 (Ido C-5), 77.8 (Gal C-2), 74.1 (Ido C-3), 73.6 (Ido $\mathrm{PhCH}_{2}$ ), $73.4\left(\right.$ Gal PhCH ${ }_{2}$ ), 73.2 (Gal $\mathrm{PhCH}_{2}$ ), 72.9 (Ido $\mathrm{PhCH}_{2}$ ), 71.8 (Ido $\mathrm{PhCH}_{2}$ ), $71.75\left(\mathrm{Gal} \mathrm{PhCH}_{2}\right.$ ), 70.6 (Gal C-6), 70.3 (Ido C-6), $68.5\left(\mathrm{Gal}\right.$ octyl $\left.\mathrm{OCH}_{2}\right), 68.1$ (Ido octyl $\left.\mathrm{OCH}_{2}\right), 31.3$ (octyl $\left.\mathrm{CH}_{2}\right), 29.5\left(\mathrm{Gal}\right.$ octyl $\left.\mathrm{CH}_{2}\right)$, 29.5 (Ido octyl $\mathrm{CH}_{2}$ ), $29.4\left(\mathrm{Gal}\right.$ octyl $\mathrm{CH}_{2}$ ), 29.3 (Ido octyl $\mathrm{CH}_{2}$ ), 29.2 (octyl $\mathrm{CH}_{2}$ ), 26.1 (octyl $\mathrm{CH}_{2}$ ), 22.7 (octyl $\mathrm{CH}_{2}$ ), 14.1 (octyl $\mathrm{CH}_{3}$ ). HRMS (ESI) calcd. for $\left(\mathrm{M}+\mathrm{Na}\right.$ ) $\mathrm{C}_{35} \mathrm{H}_{46} \mathrm{O}_{6} \mathrm{Na}$ : 585.3187, found: 585.3184 .

n-Octyl 3-O-benzyl- $\alpha$-D-galactofuranoside

2-O-benzyl-a-D-idofuranoside (69). Compound 54 was subjected to the general epoxide 
opening procedure, yielding 68 and 69 as colorless oils. 68: $R_{f} 0.30$ (1:1, hexanes:EtOAc); $[\alpha]_{\mathrm{D}}$ $+30.8\left(c 1.0, \mathrm{CH}_{2} \mathrm{Cl}_{2}\right) ;{ }^{1} \mathrm{H}$ NMR $\left(500 \mathrm{MHz}, \mathrm{CD}_{3} \mathrm{OD}, \delta_{\mathrm{H}}\right) 7.38-7.25(\mathrm{~m}, 5 \mathrm{H}, \mathrm{Ar}), 4.87(\mathrm{~d}, 1 \mathrm{H}, J$ $=4.6 \mathrm{~Hz}, \mathrm{H}-1), 4.82\left(\mathrm{~d}, 1 \mathrm{H}, J=11.6 \mathrm{~Hz}, \mathrm{PhCH}_{2}\right), 4.62\left(\mathrm{~d}, 1 \mathrm{H}, J=11.6 \mathrm{~Hz}, \mathrm{PhCH}_{2}\right), 4.17(\mathrm{dd}, 1$ $\mathrm{H}, J=6.8,4.6 \mathrm{~Hz}, \mathrm{H}-2), 4.09$ (dd, $1 \mathrm{H}, J=6.8,6.2 \mathrm{~Hz}, \mathrm{H}-3), 3.89$ (dd, $1 \mathrm{H}, J=6.2,5.0 \mathrm{~Hz}, \mathrm{H}-4$ ), 3.80 (ddd, $1 \mathrm{H}, J=9.6,6.9,6.9 \mathrm{~Hz}$, octyl $\left.\mathrm{OCH}_{2}\right), 3.62-3.54$ (m, $\left.2 \mathrm{H}, \mathrm{H}-5, \mathrm{H}-6\right), 3.51-3.45$ (m, 2 H, H-6, octyl $\left.\mathrm{OCH}_{2}\right), 1.65-1.58$ (m, $2 \mathrm{H}$, octyl $\left.\mathrm{CH}_{2}\right), 1.40-1.25$ (m, $10 \mathrm{H}$, octyl $\left.\mathrm{CH}_{2}\right), 0.95-0.85$ (m, $3 \mathrm{H}$, octyl $\left.\mathrm{CH}_{3}\right) ;{ }^{13} \mathrm{C}$ NMR $\left(125 \mathrm{MHz}, \mathrm{CD}_{3} \mathrm{OD}, \delta_{\mathrm{C}}\right) 139.6(\mathrm{Ar}), 129.3(\mathrm{Ar} \times 2), 129.0(\mathrm{Ar} \times$ 2), 128.7 (Ar), 103.5 (C-1), 84.1 (C-3), 82.6 (C-4), 79.0 (C-2), 74.5 (C-5), $73.1\left(\mathrm{PhCH}_{2}\right), 70.0$ (octyl $\mathrm{OCH}_{2}$ ), 64.1 (C-6), 33.0 (octyl $\mathrm{CH}_{2}$ ), 30.7 (octyl $\mathrm{CH}_{2}$ ), 30.6 (octyl $\mathrm{CH}_{2}$ ), 30.4 (octyl $\mathrm{CH}_{2}$ ), 27.2 (octyl $\mathrm{CH}_{2}$ ), 23.7 (octyl $\mathrm{CH}_{2}$ ), 14.4 (octyl $\mathrm{CH}_{3}$ ). HRMS (ESI) calcd. for $(\mathrm{M}+\mathrm{Na}$ ) $\mathrm{C}_{21} \mathrm{H}_{34} \mathrm{O}_{6} \mathrm{Na}: 405.2248$, found: 405.2246. 69: $R_{f} 0.13$ (1:1, hexanes:EtOAc); $[\alpha]_{\mathrm{D}}+19.6(c 1.4$, $\left.\mathrm{CH}_{2} \mathrm{Cl}_{2}\right) ;{ }^{1} \mathrm{H}$ NMR $\left(500 \mathrm{MHz}, \mathrm{CD}_{3} \mathrm{OD}, \delta_{\mathrm{H}}\right) 7.35-7.27(\mathrm{~m}, 5 \mathrm{H}, \mathrm{Ar}), 4.97(\mathrm{~d}, 1 \mathrm{H}, J=1.5 \mathrm{~Hz}$, H-1), 4.64 (d, $\left.1 \mathrm{H}, J=11.9 \mathrm{~Hz}, \mathrm{PhCH}_{2}\right), 4.60$ (d, $\left.1 \mathrm{H}, J=11.9 \mathrm{~Hz}, \mathrm{PhCH}_{2}\right), 4.24$ (dd, $1 \mathrm{H}, J=$ 5.3, 2.4 Hz, H-3), 4.15 (dd, 1 H, $J=5.3,5.2 \mathrm{~Hz}, \mathrm{H}-4$ ), 3.92-3.90 (m, $1 \mathrm{H}, \mathrm{H}-2$ ), 3.88 (ddd, $1 \mathrm{H}, J$ $=5.9,5.4,5.2 \mathrm{~Hz}, \mathrm{H}-5), 3.77\left(\mathrm{ddd}, 1 \mathrm{H}, J=9.6,6.7,6.7 \mathrm{~Hz}\right.$, octyl $\left.\mathrm{OCH}_{2}\right), 3.65(\mathrm{dd}, 1 \mathrm{H}, J=11.2$, $5.4 \mathrm{~Hz}, \mathrm{H}-6), 3.60$ (dd, $1 \mathrm{H}, J=11.2,5.9 \mathrm{~Hz}, \mathrm{H}-6), 3.42$ (ddd, $1 \mathrm{H}, J=9.6,6.6,6.6 \mathrm{~Hz}$, octyl $\left.\mathrm{OCH}_{2}\right), 1.60-1.52\left(\mathrm{~m}, 2 \mathrm{H}\right.$, octyl $\left.\mathrm{CH}_{2}\right), 1.38-1.27$ (m, $10 \mathrm{H}$, octyl $\left.\mathrm{CH}_{2}\right), 0.91-0.87$ (m, $3 \mathrm{H}$, octyl $\left.\mathrm{CH}_{3}\right) ;{ }^{13} \mathrm{C}$ NMR $\left(125 \mathrm{MHz}, \mathrm{CD}_{3} \mathrm{OD}, \delta_{\mathrm{C}}\right) 139.3(\mathrm{Ar}), 129.4(\mathrm{Ar} \times 2), 129.0(\mathrm{Ar} \times 2), 128.8(\mathrm{Ar})$, 108.0 (C-1), 89.8 (C-2), 83.1 (C-4), 75.8 (C-3), $73.0\left(\mathrm{PhCH}_{2}\right), 72.3$ (C-5), 69.6 (octyl $\mathrm{OCH}_{2}$ ), 64.4 (C-6), 33.0 (octyl $\mathrm{CH}_{2}$ ), 30.6 (octyl $\mathrm{CH}_{2}$ ), 30.5 (octyl $\mathrm{CH}_{2}$ ), 30.4 (octyl $\mathrm{CH}_{2}$ ), 27.3 (octyl 
$\mathrm{CH}_{2}$ ), 23.7 (octyl $\mathrm{CH}_{2}$ ), 14.4 (octyl $\mathrm{CH}_{3}$ ). HRMS (ESI) calcd. for (M $+\mathrm{Na}$ ) $\mathrm{C}_{21} \mathrm{H}_{34} \mathrm{O}_{6} \mathrm{Na}$ : 405.2248, found: 405.2248 .

\section{References for Supporting Information}

1. Callam, C. S.; Gadikota, R. R.; Lowary, T. L. J. Org. Chem. 2001, 66, 4549-4558.

2. Ferrières, V.; Bertho, J.-N.; Plusquellec, D. Carbohydr. Res. 1998, 311, 25-35.

3. (a) Patt, S.; Shoolery, J. N. J. Magn. Reson. 1982, 46, 535-539; (b) Torres, A. M.; Nakashima, T. T.; McClung, R. E. D. J. Magn. Reson. Ser. A 1993, 101, 285-289. 


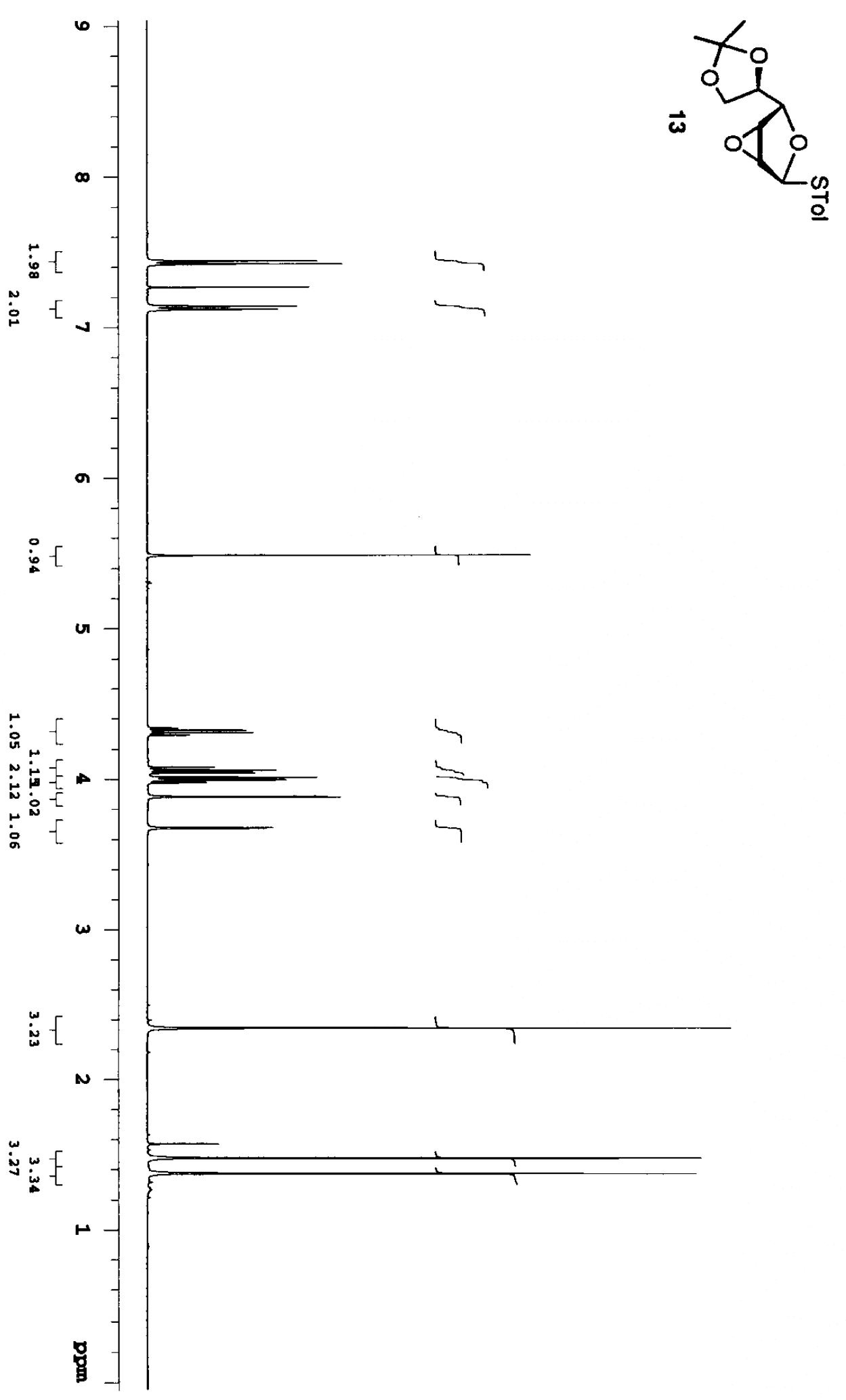

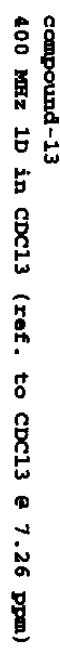

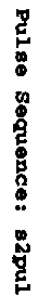



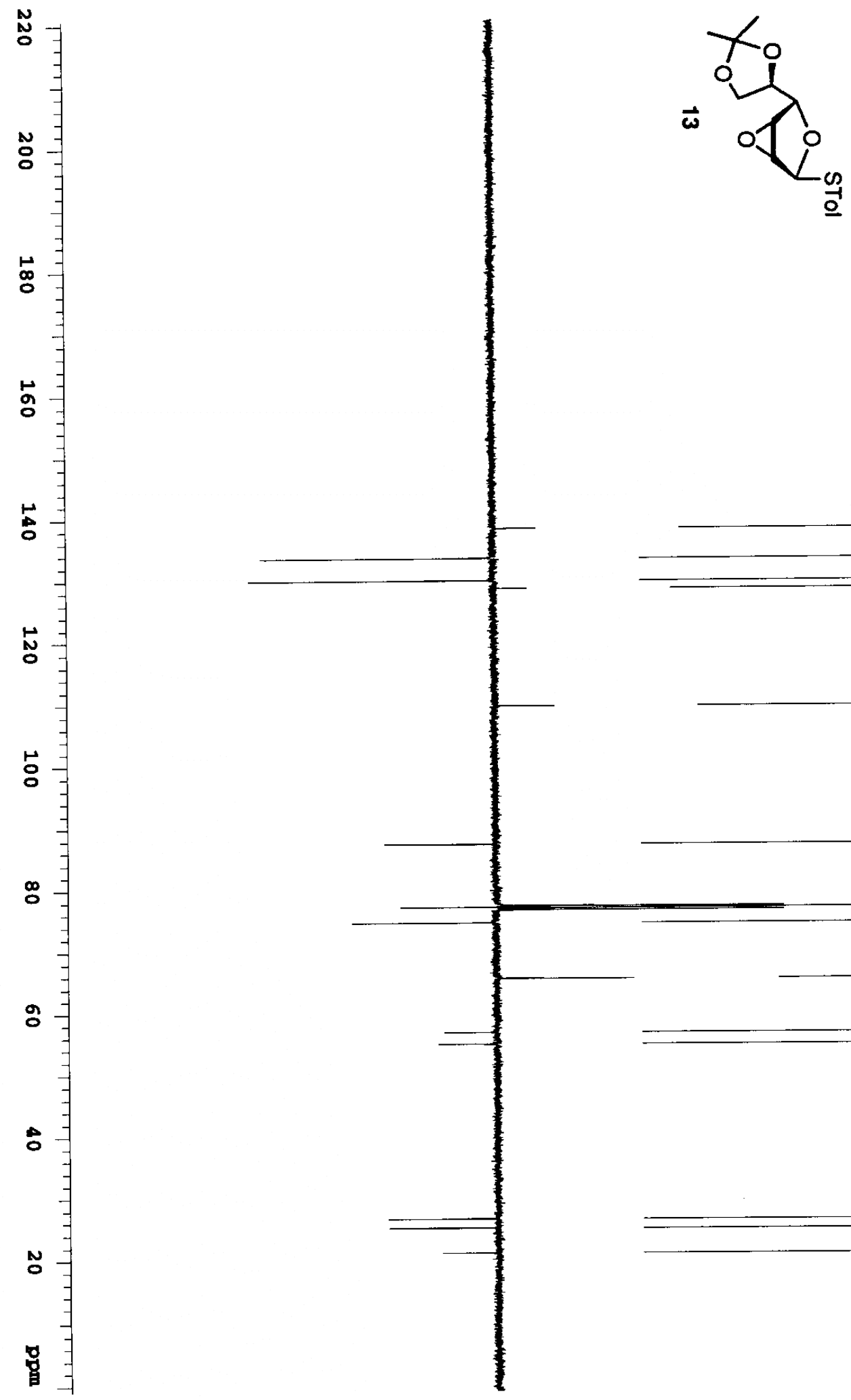

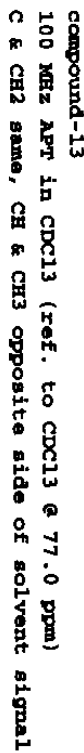

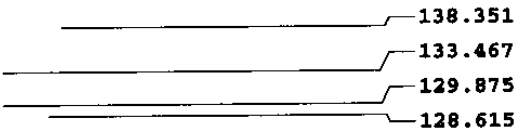

109.711

87.273
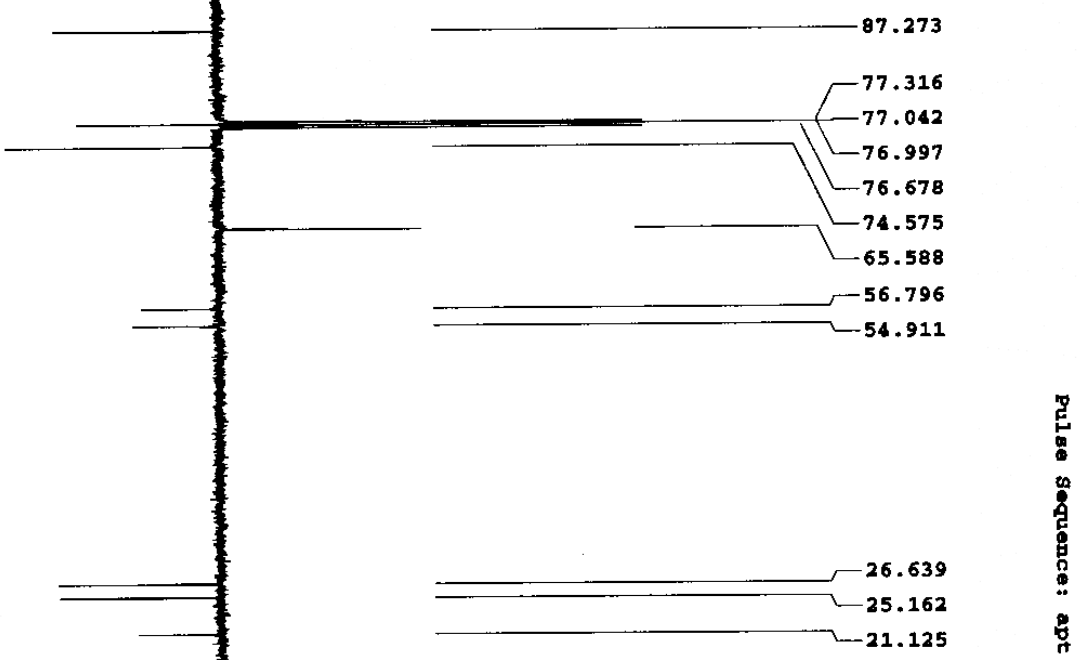


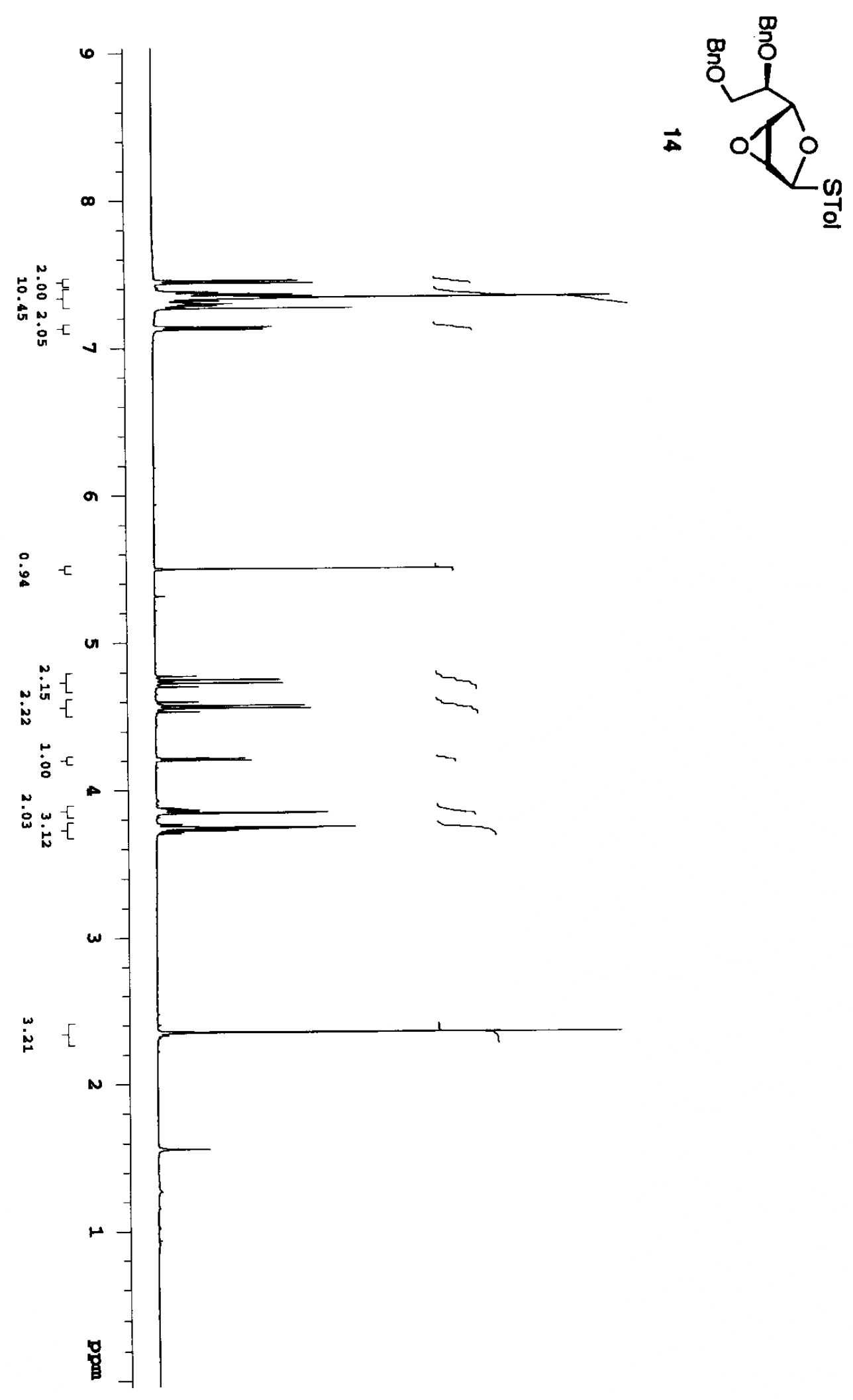

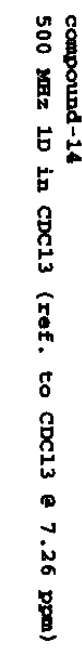




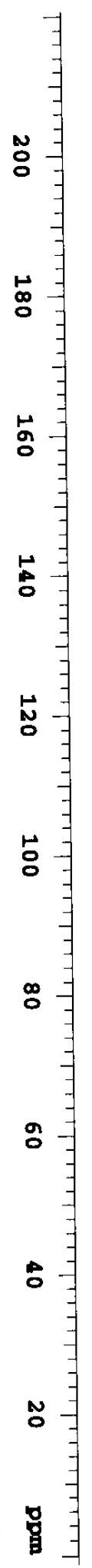

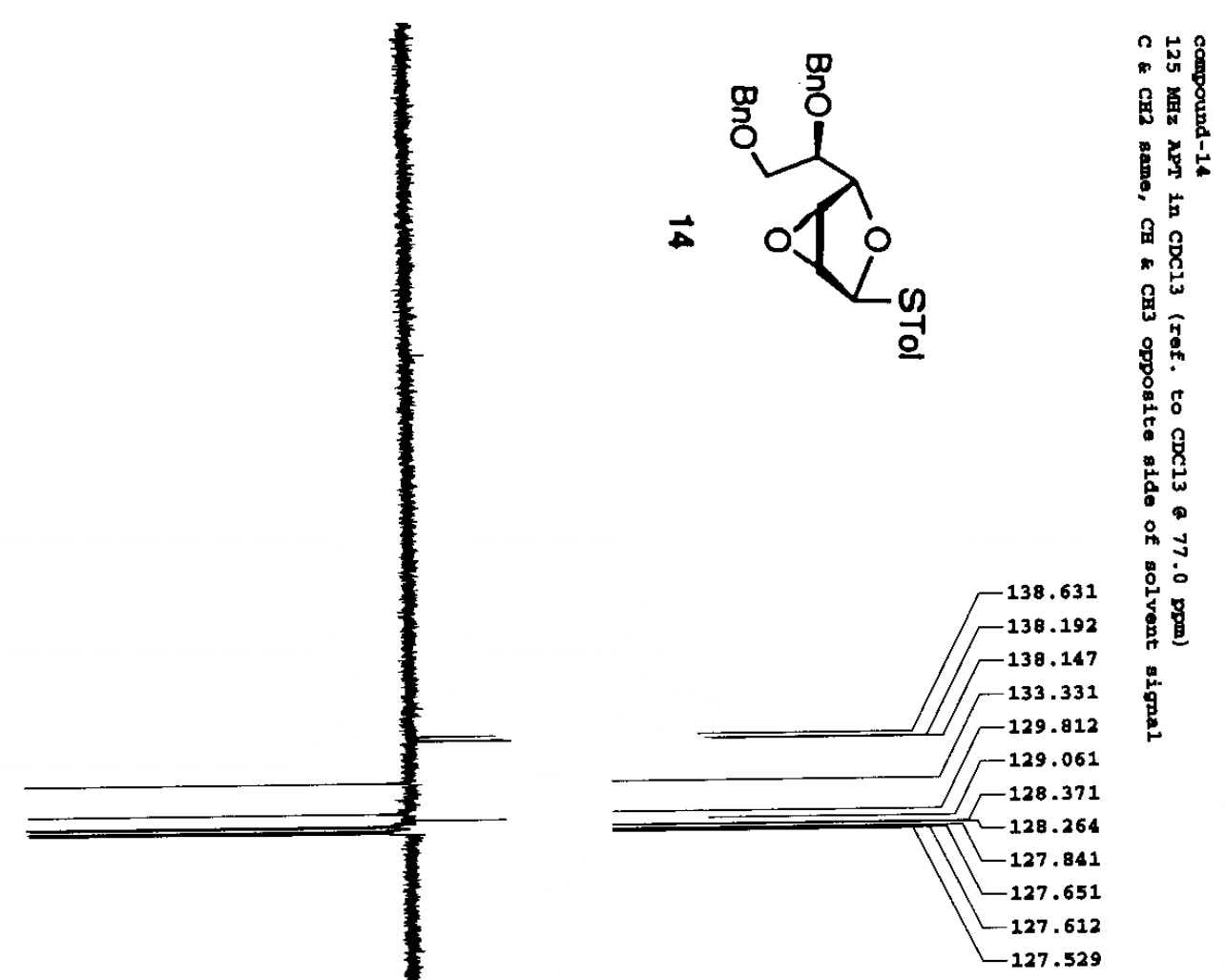




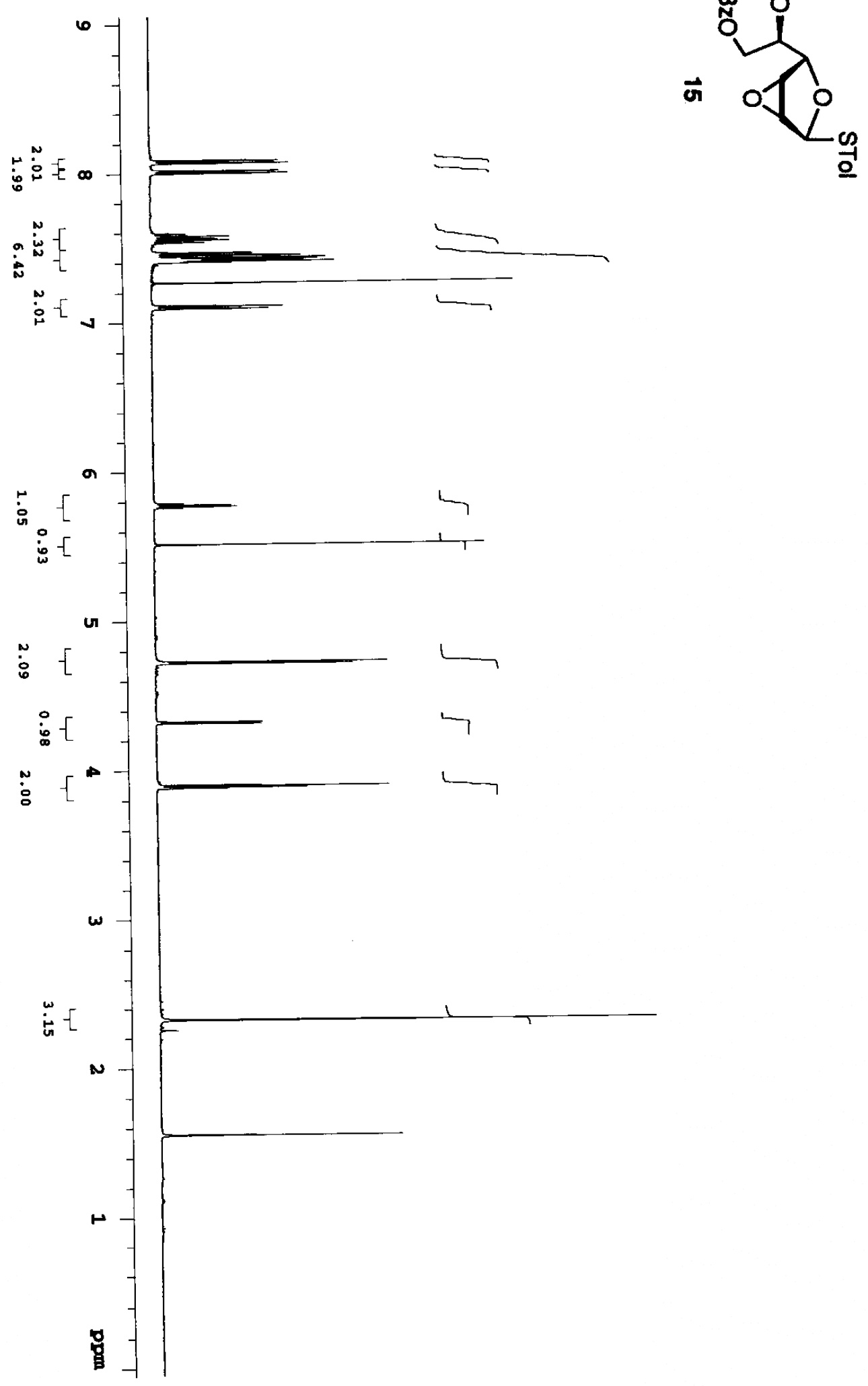




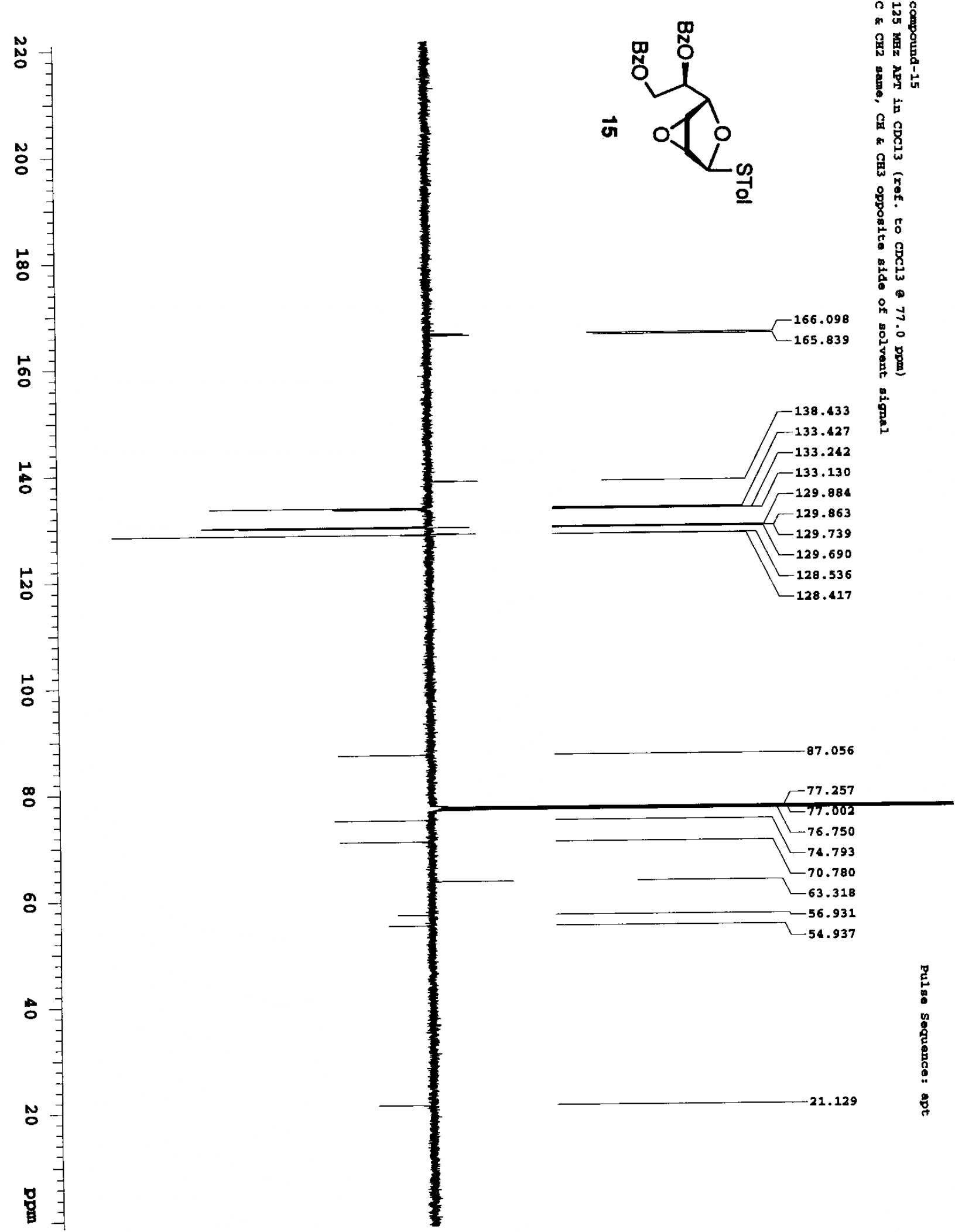



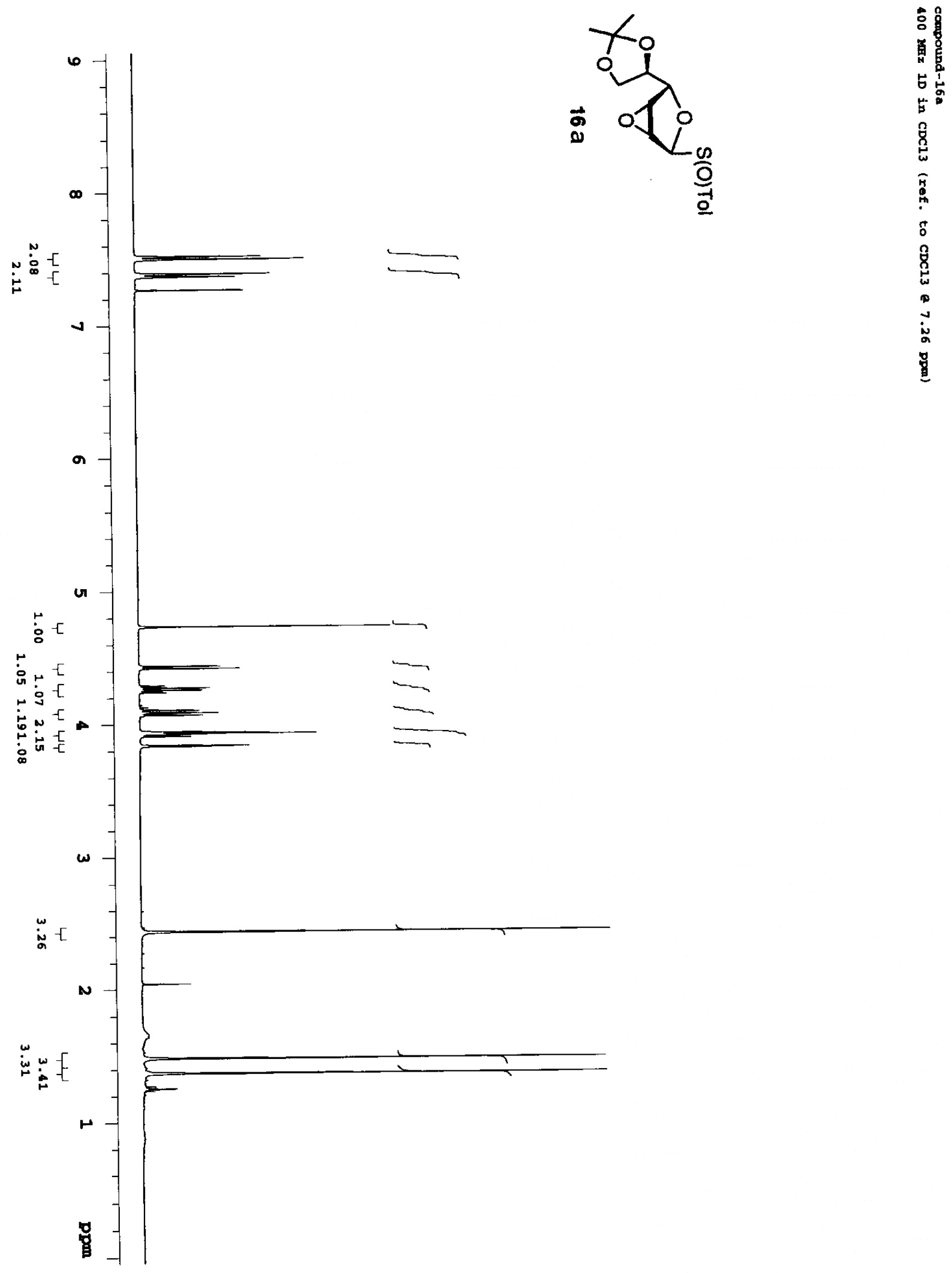

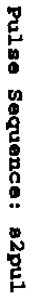




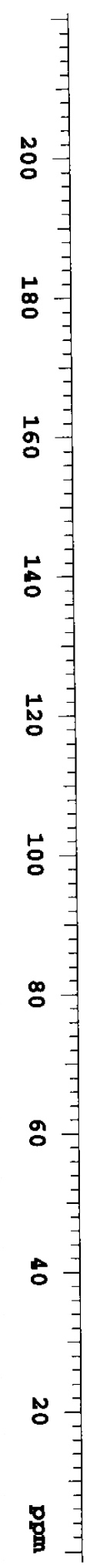

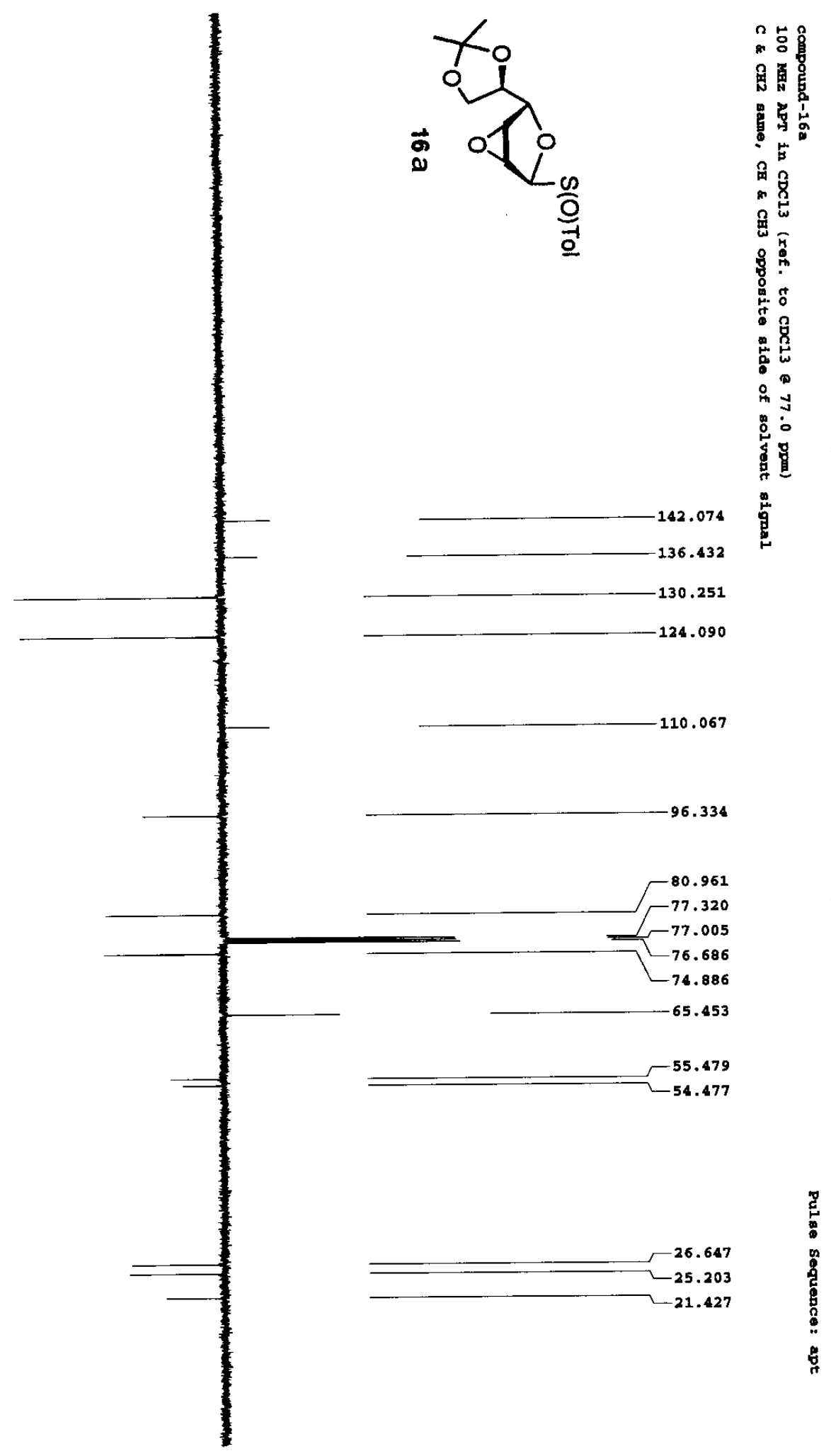



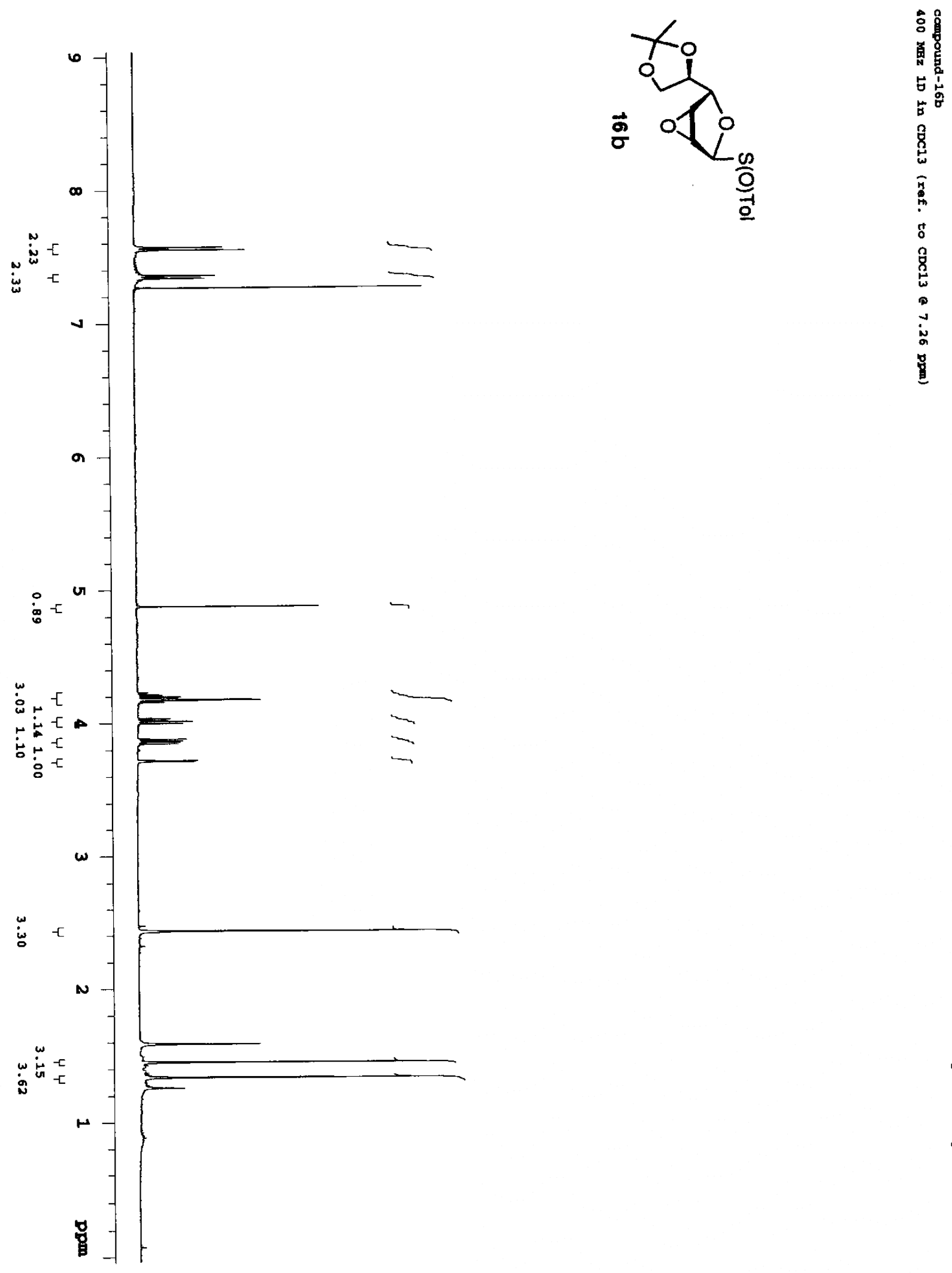

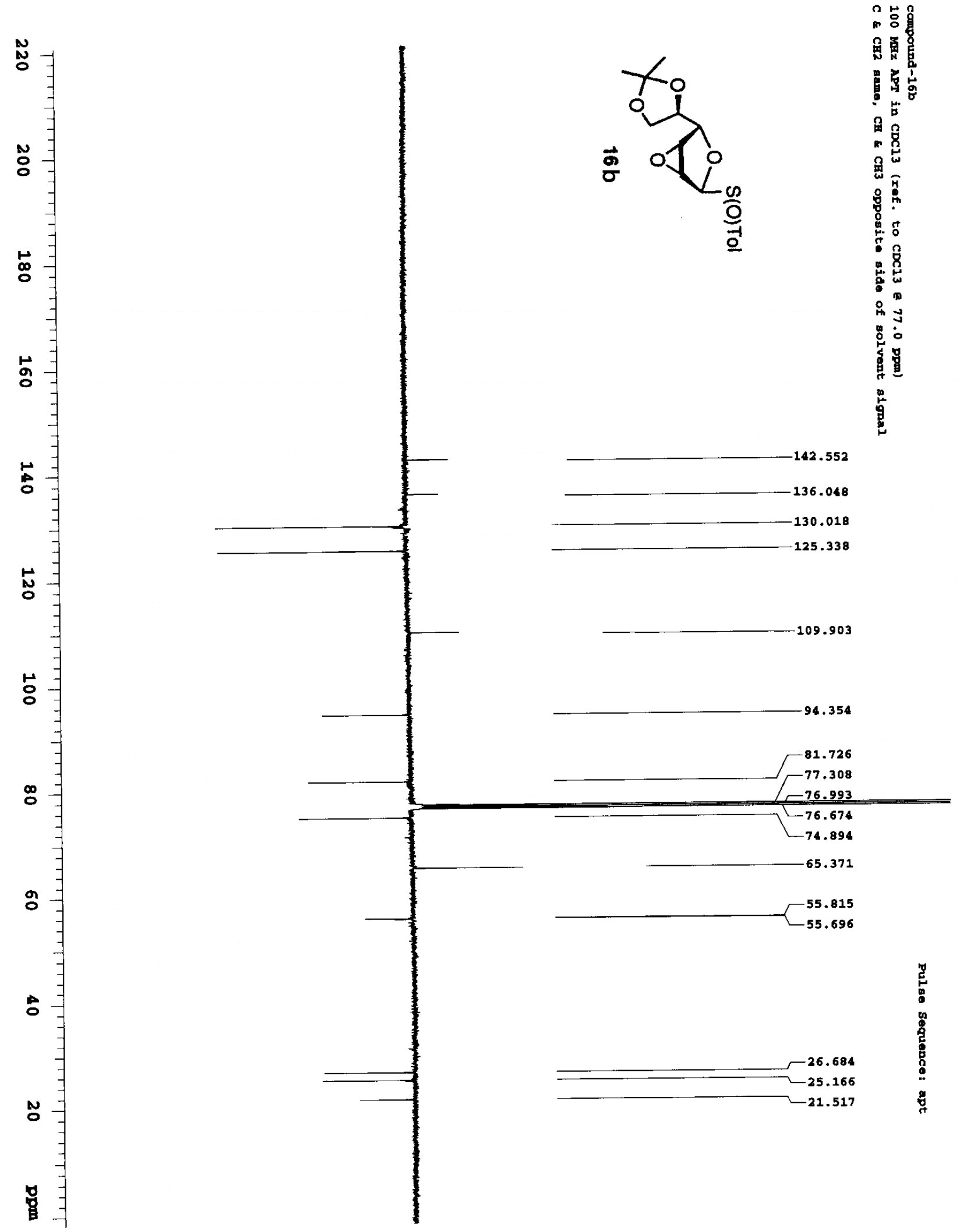


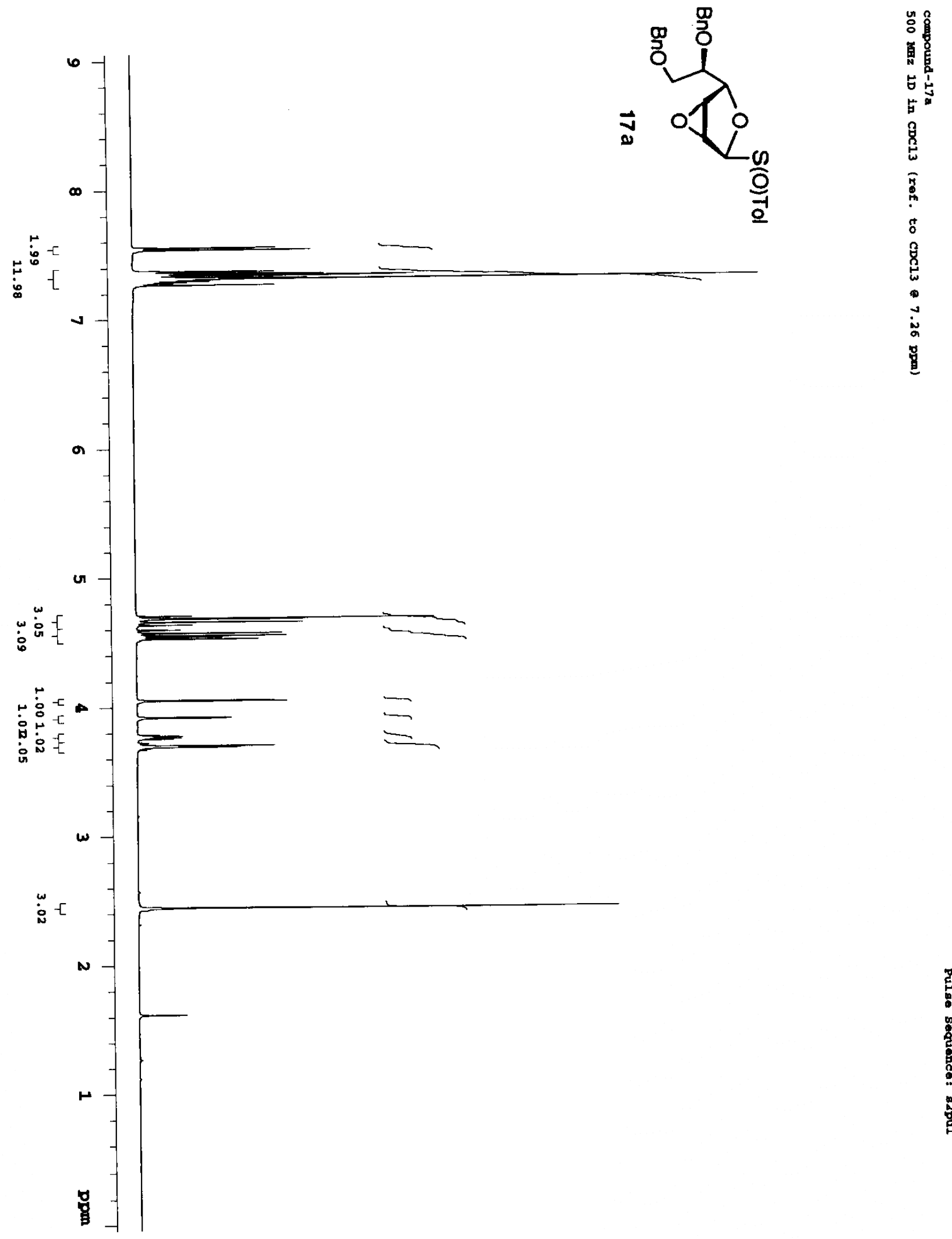




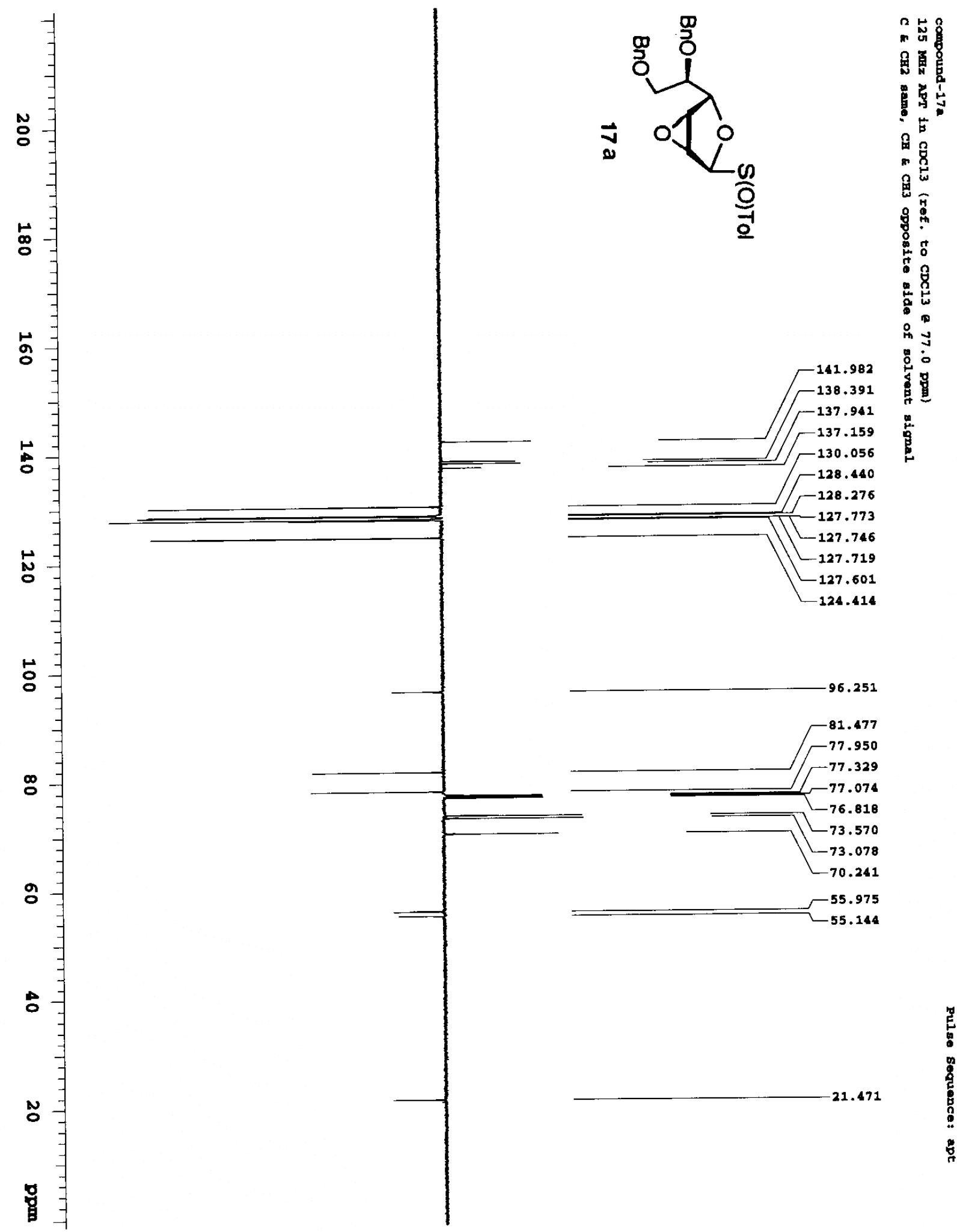




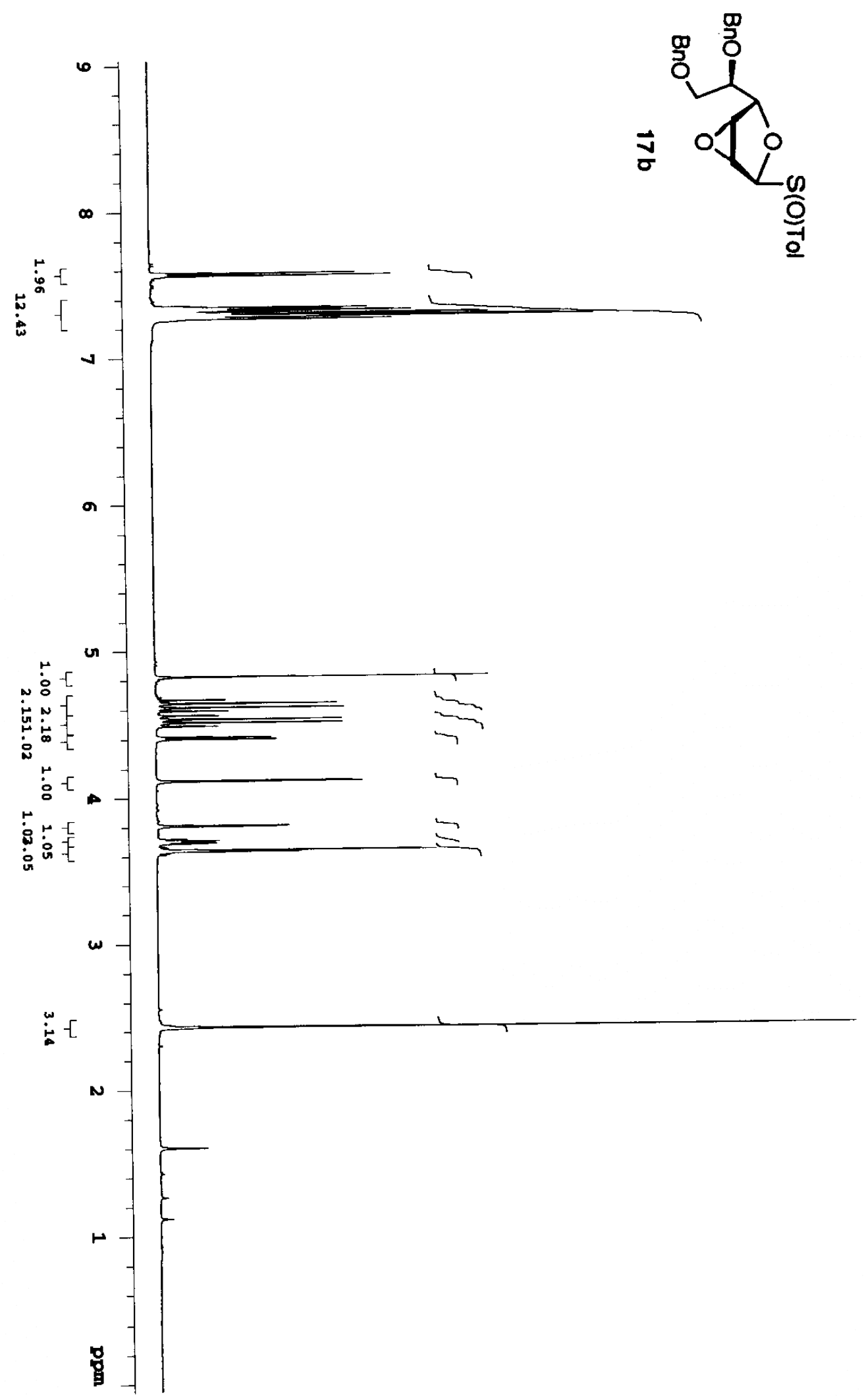

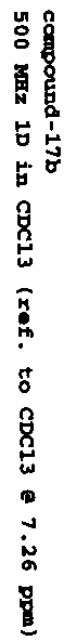



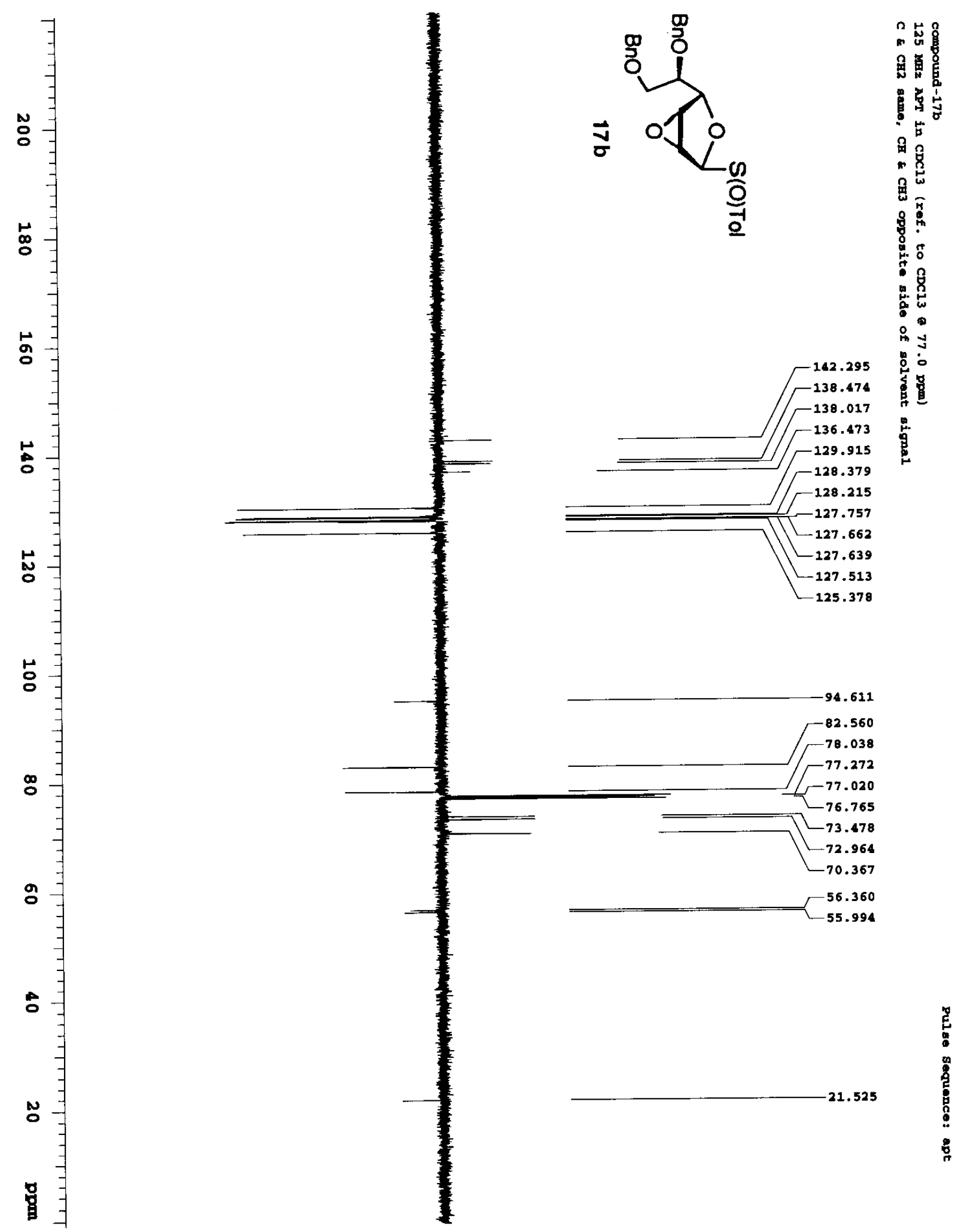


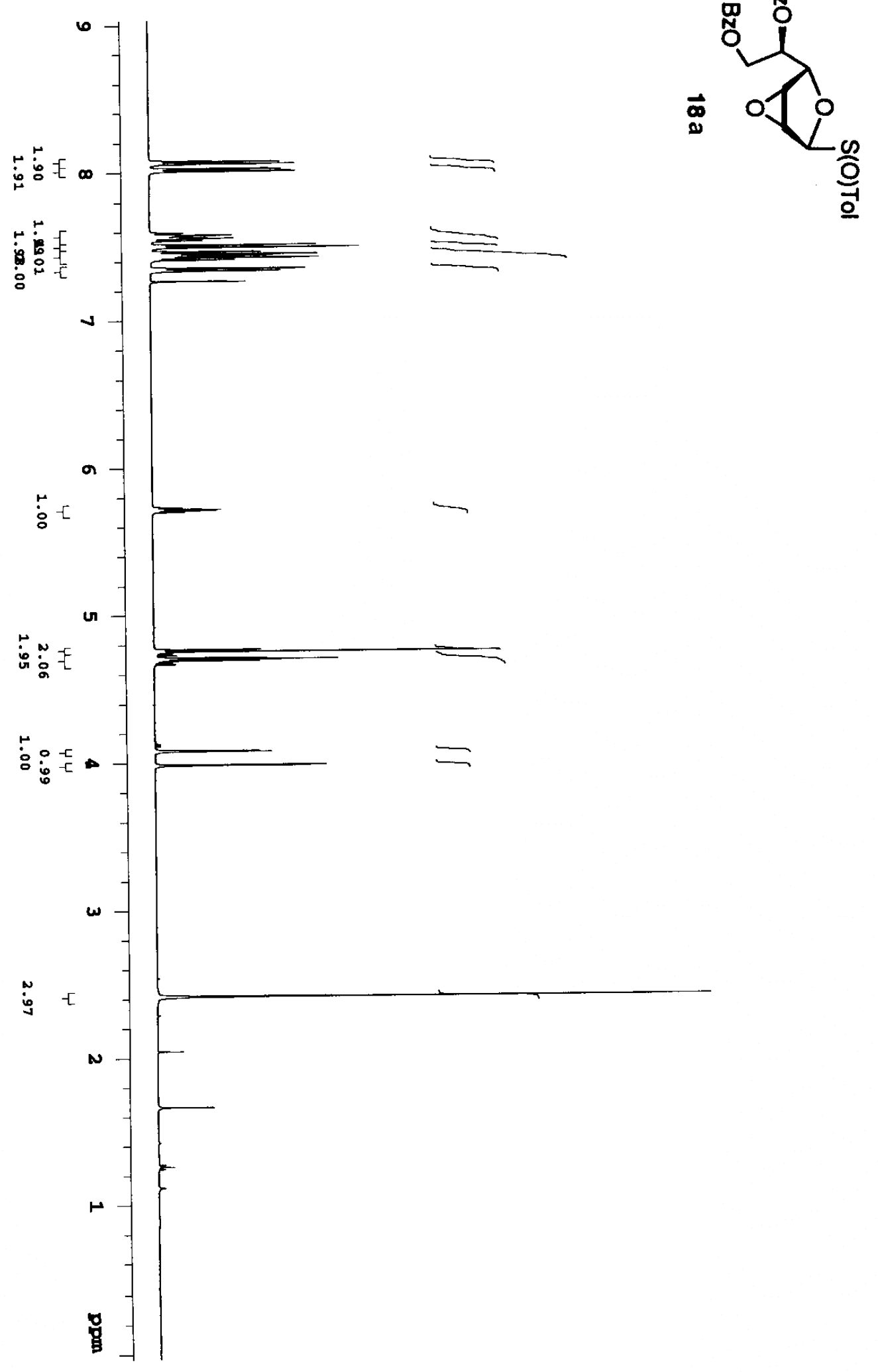

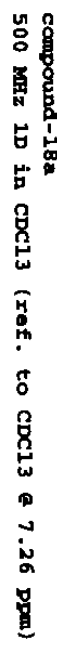




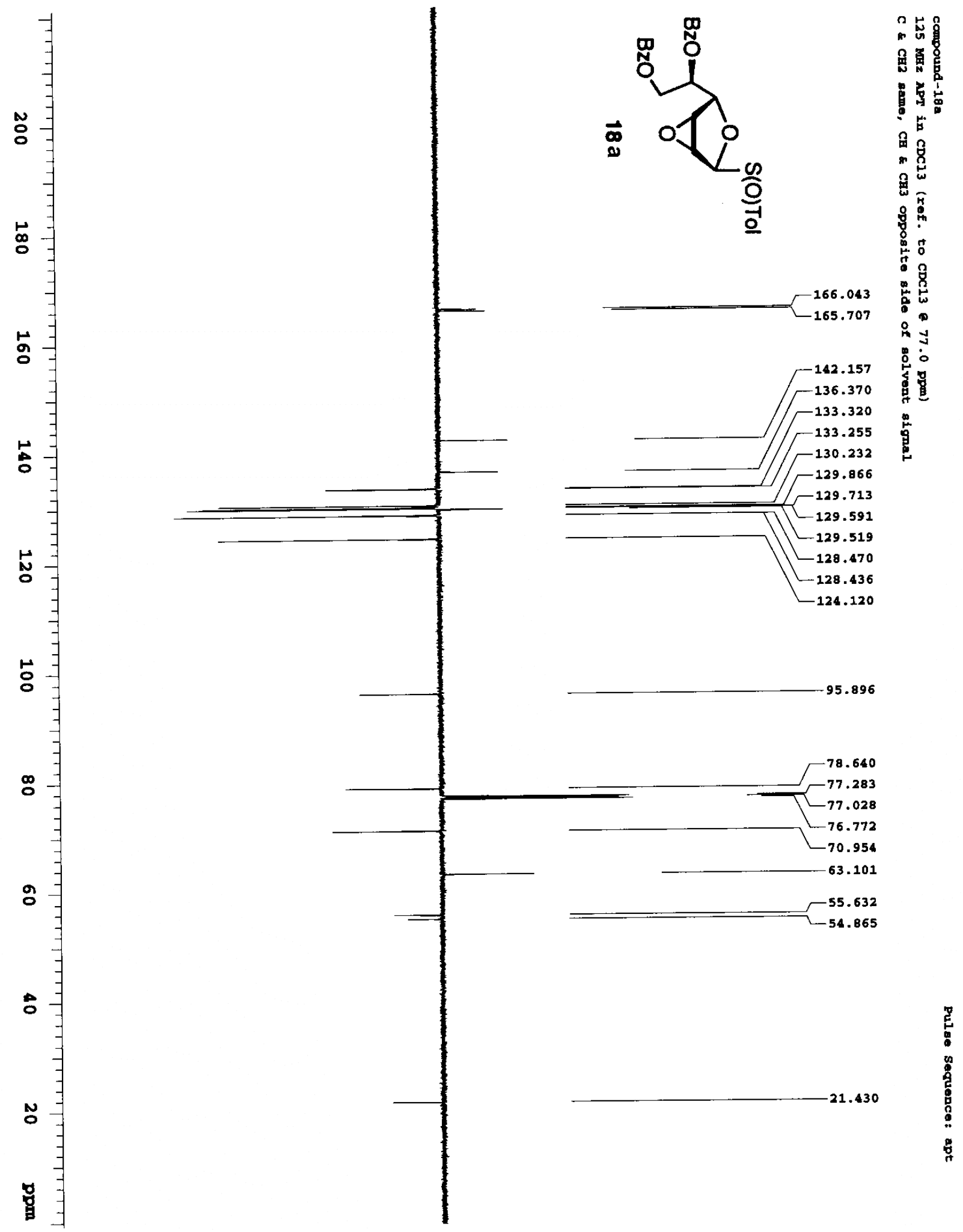



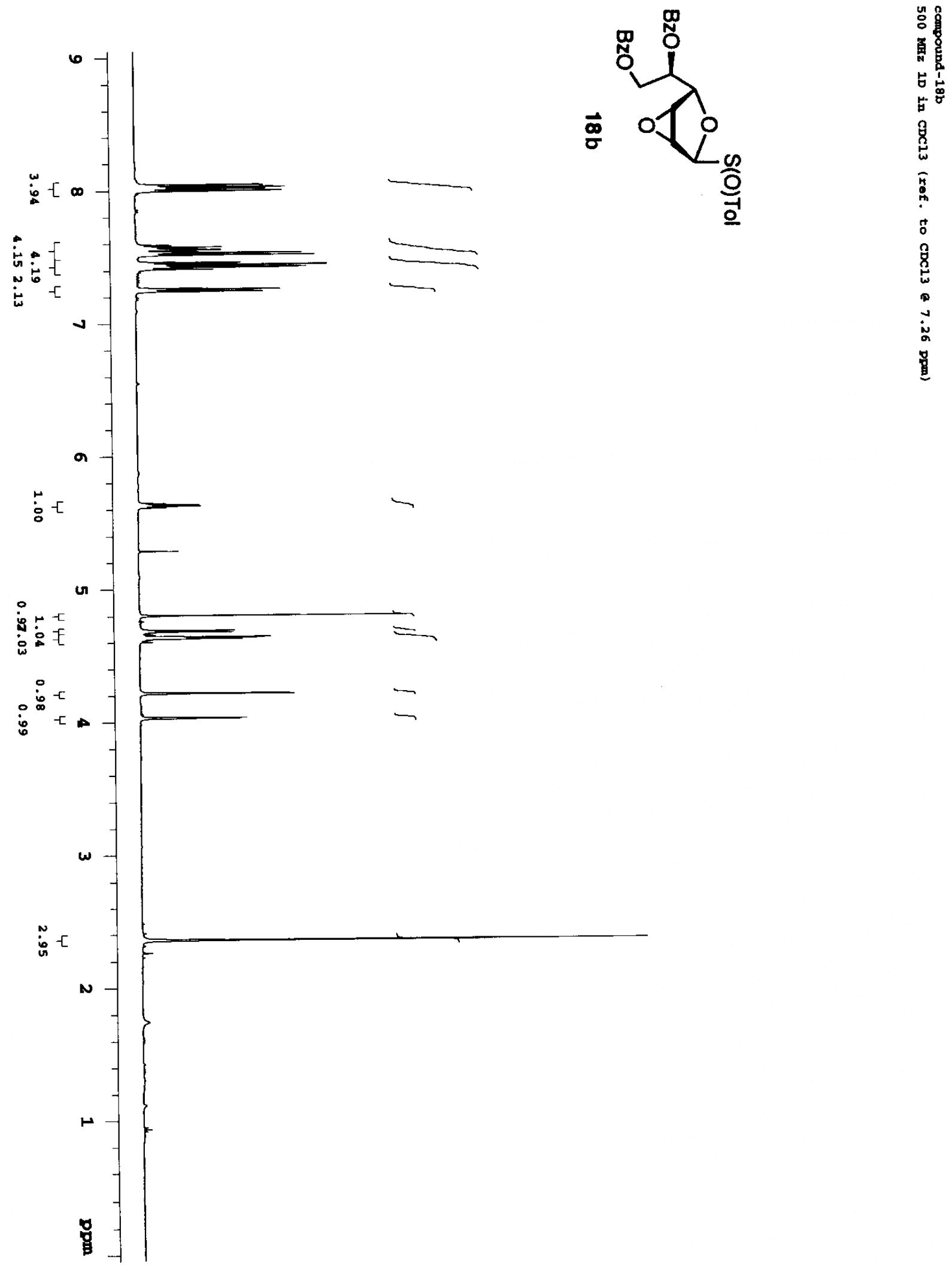


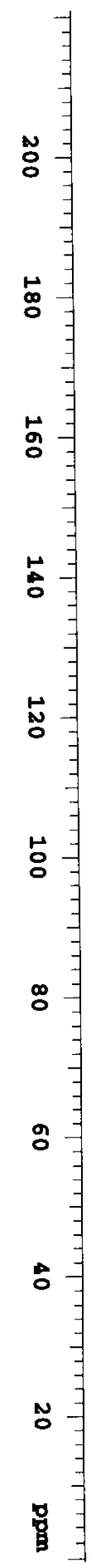

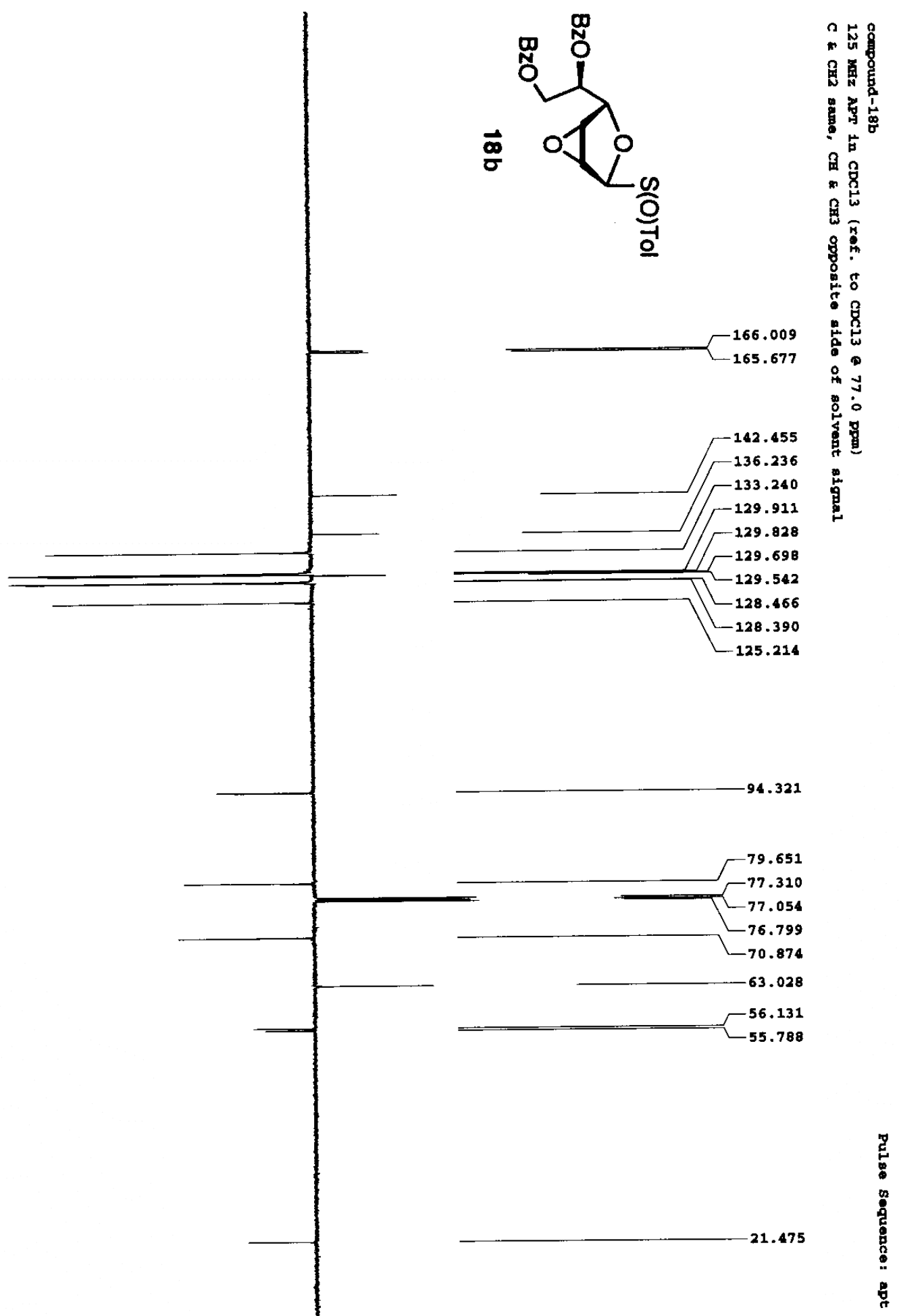



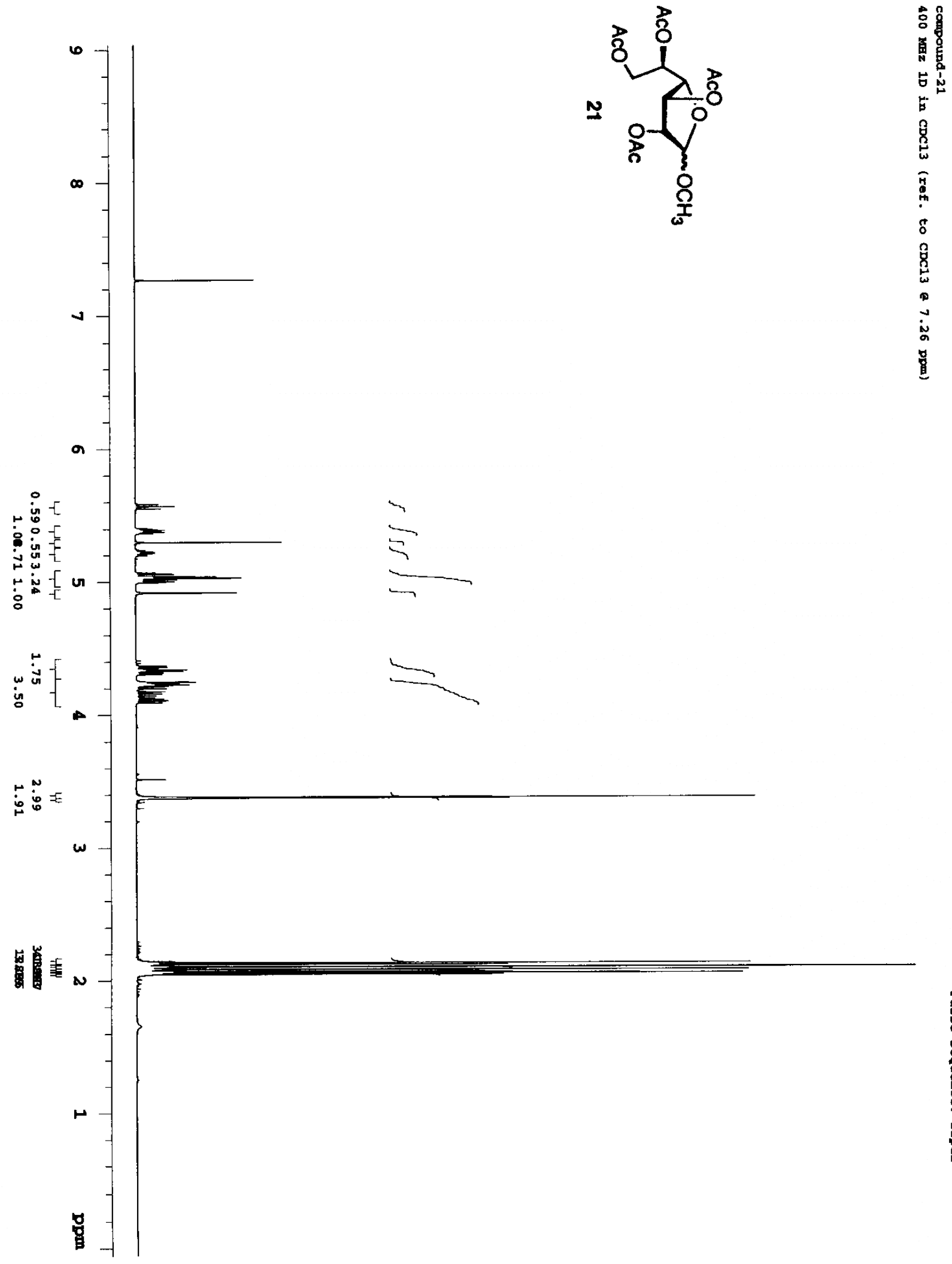

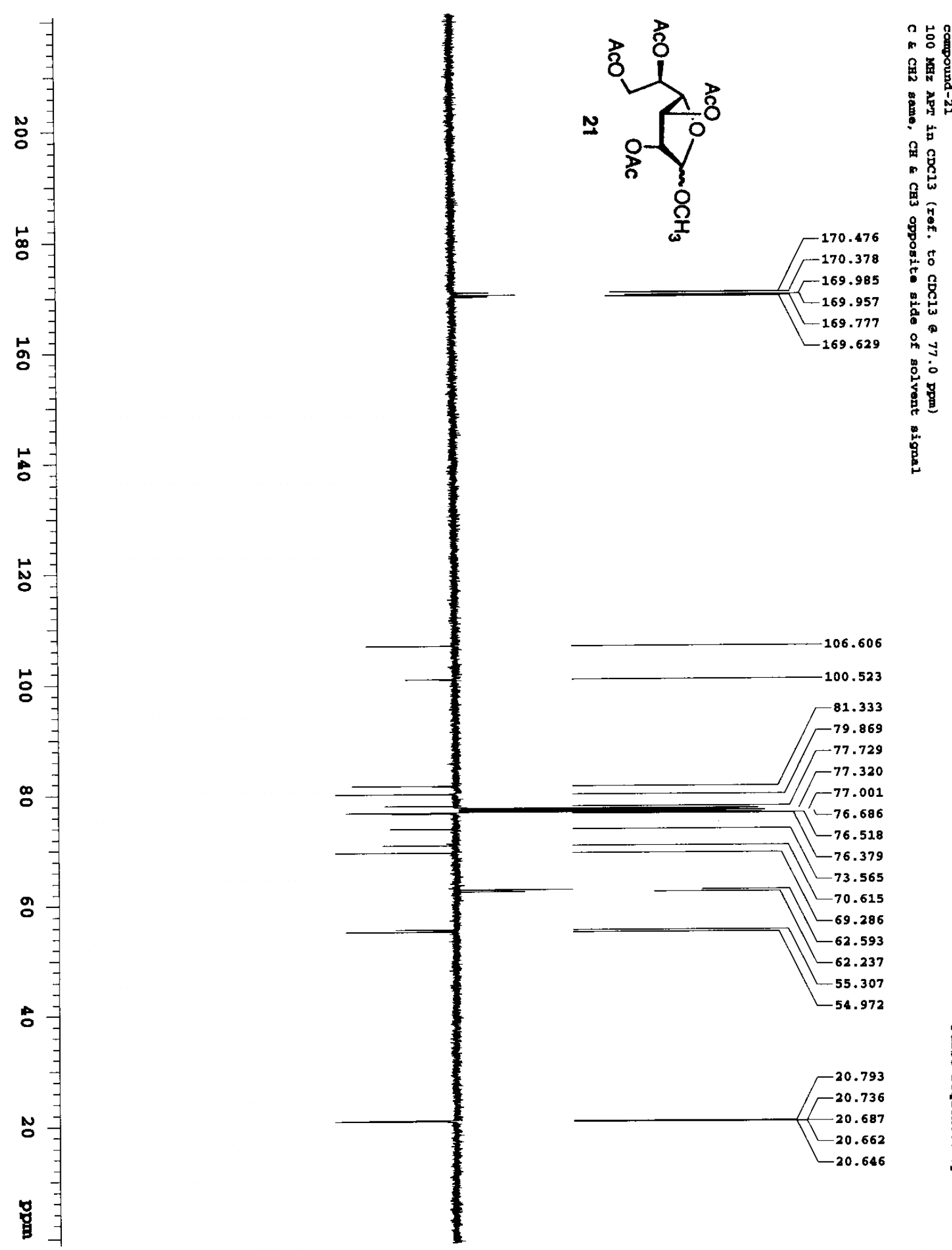

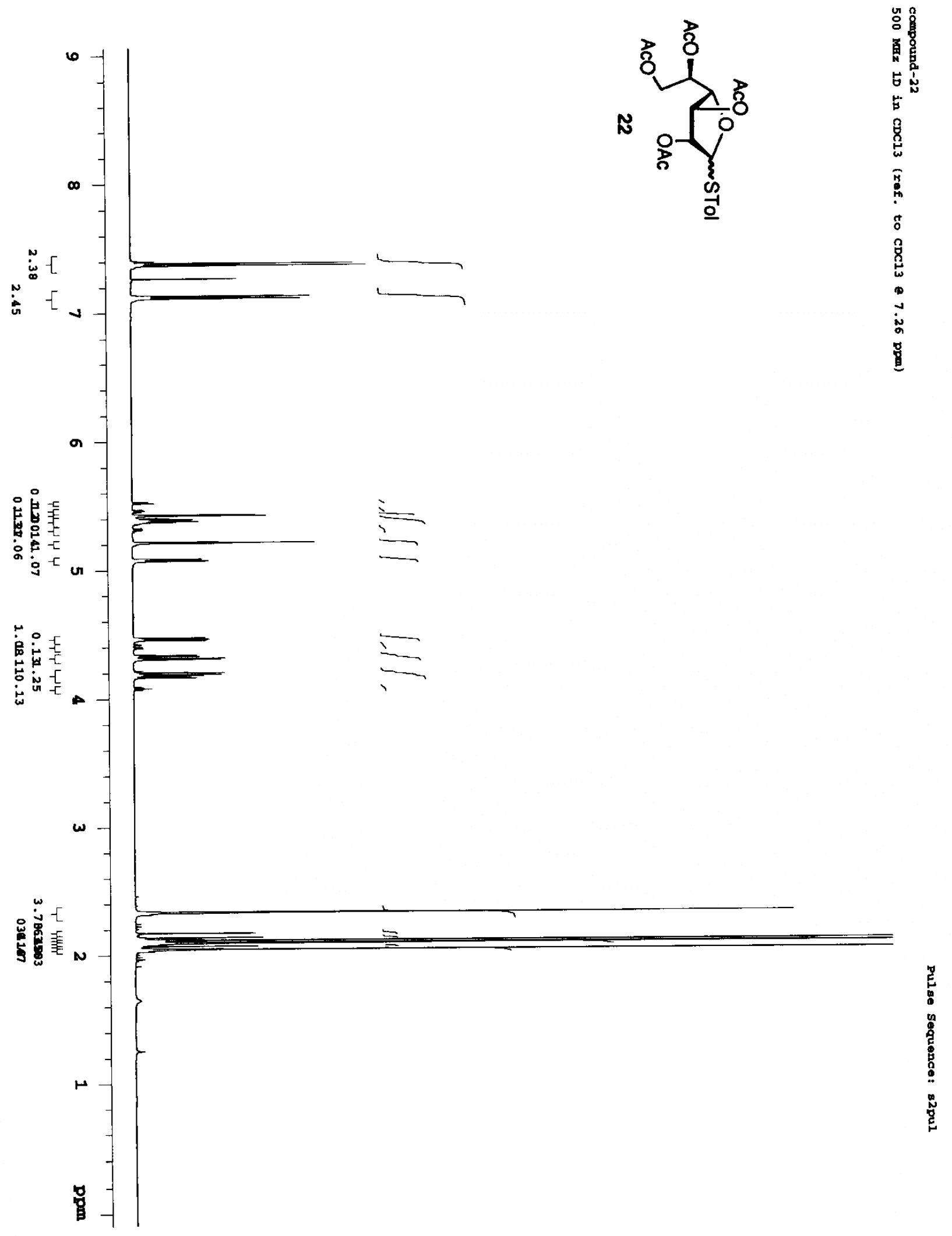


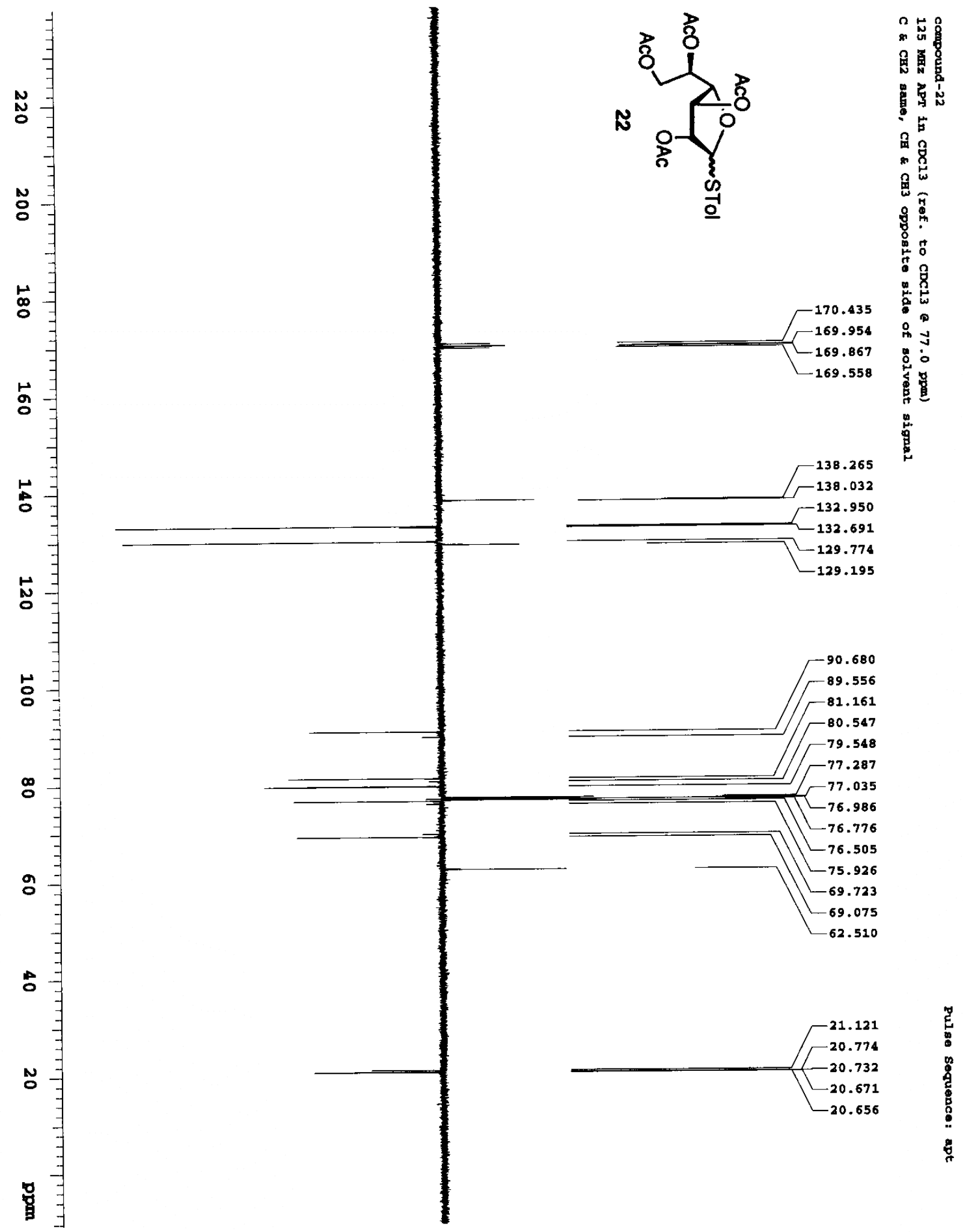



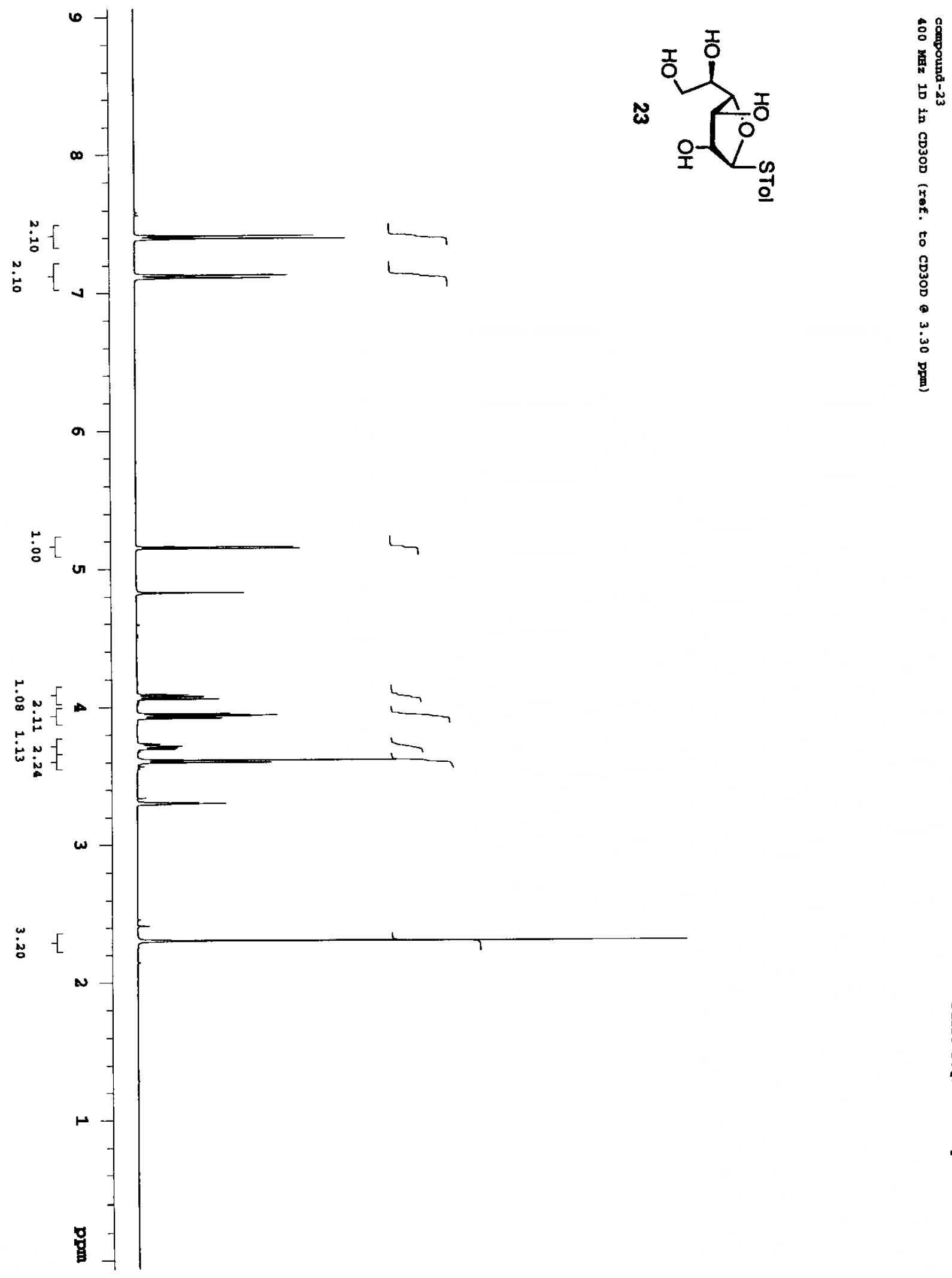

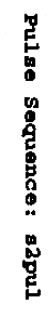




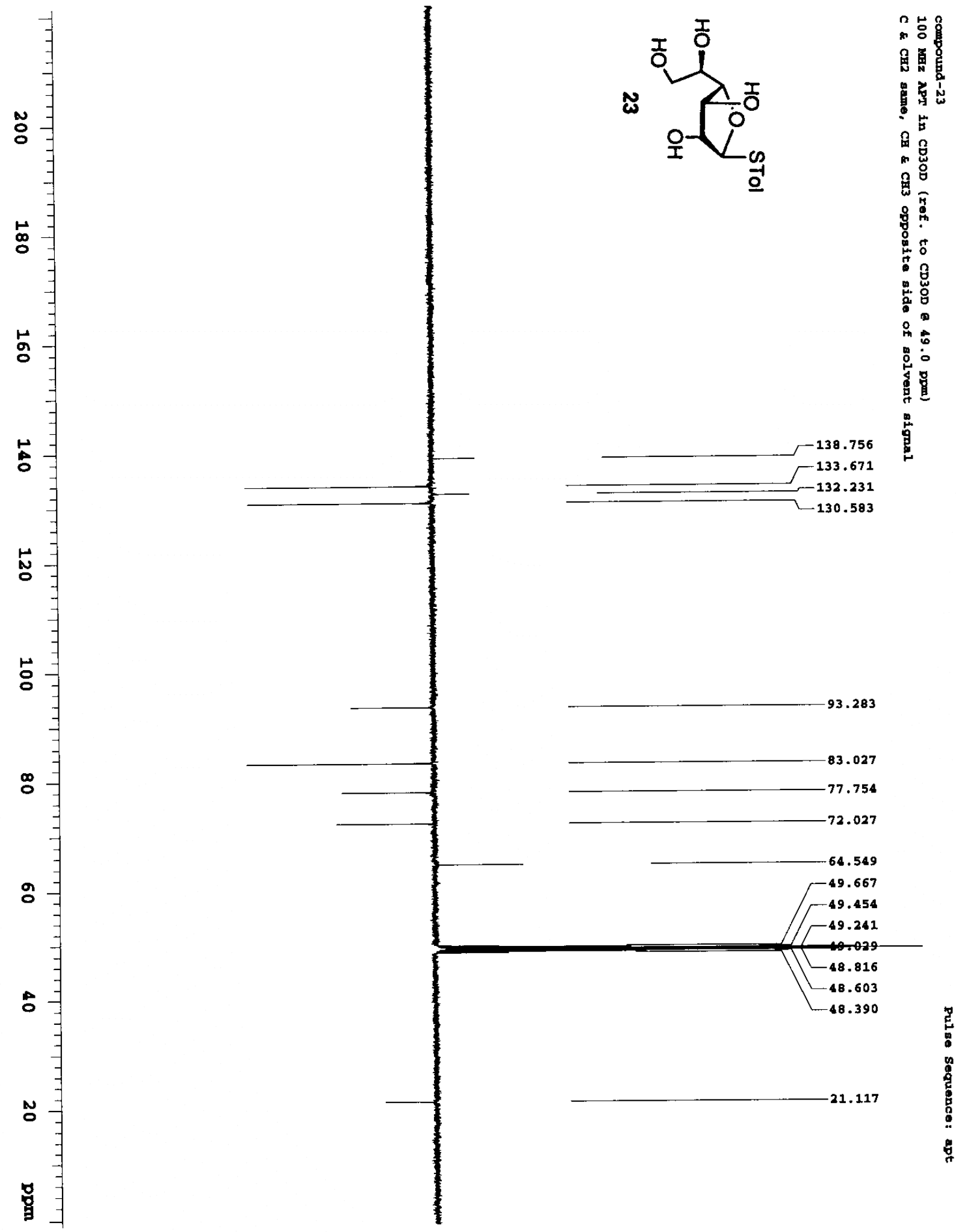




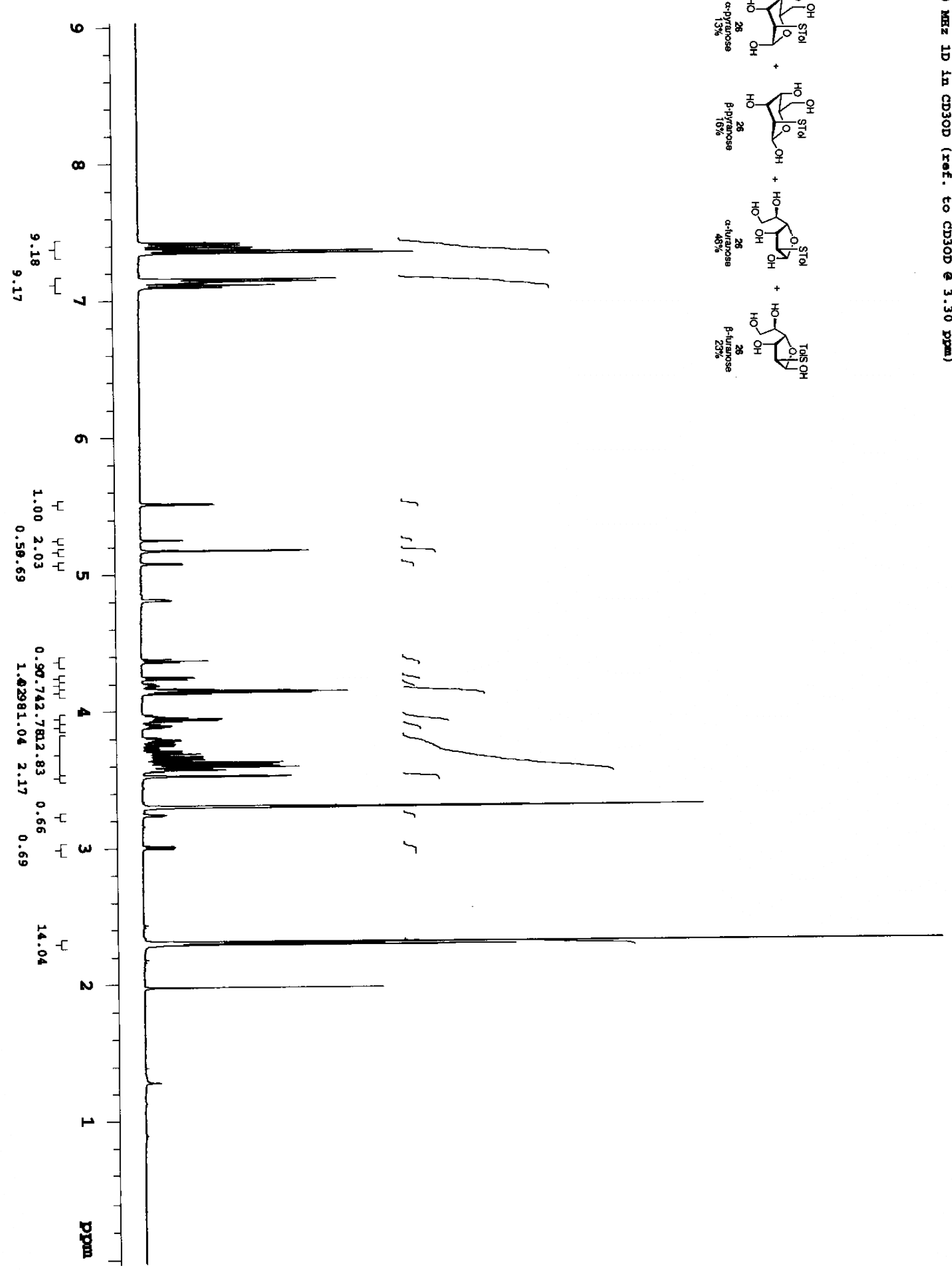

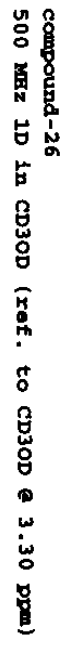



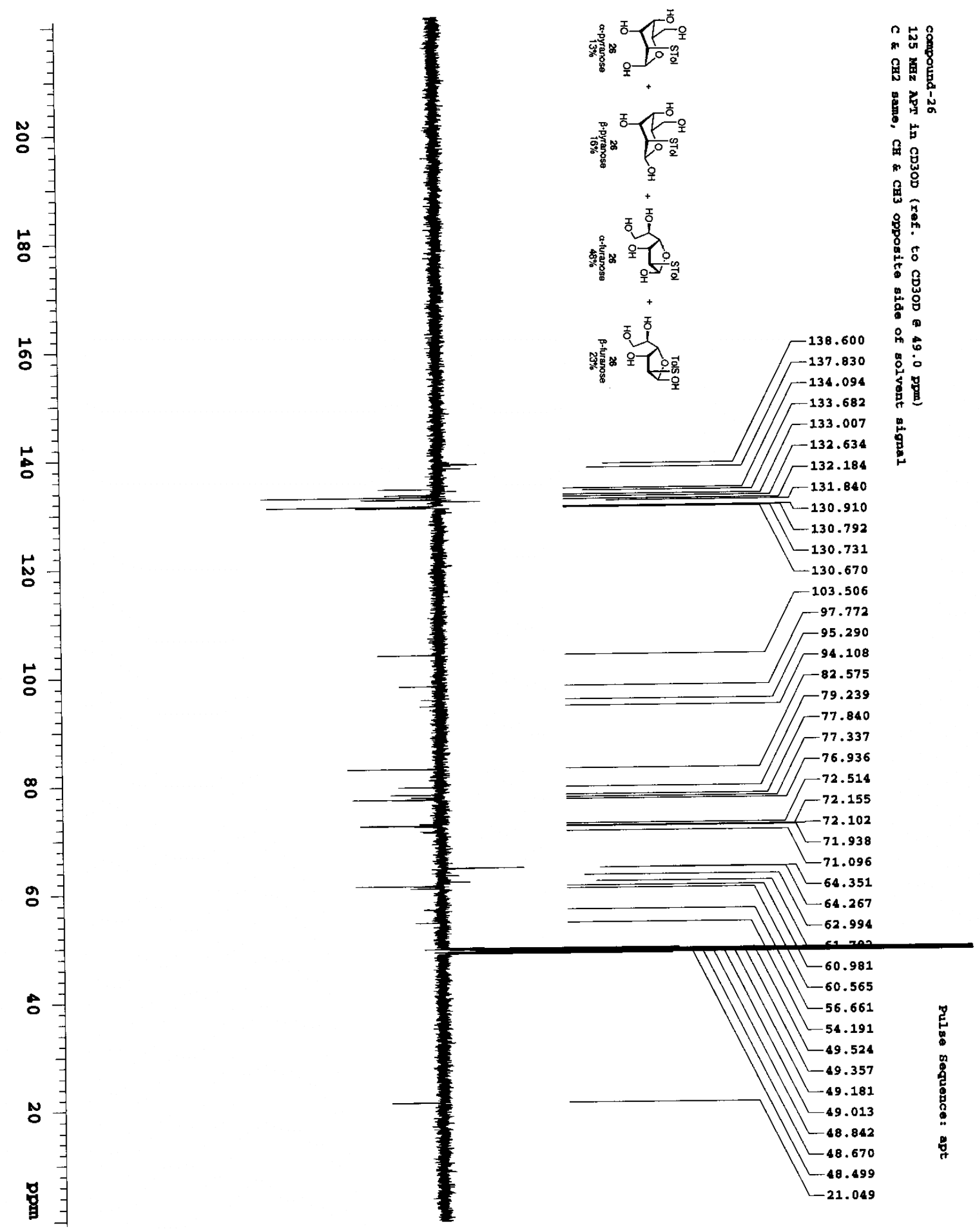


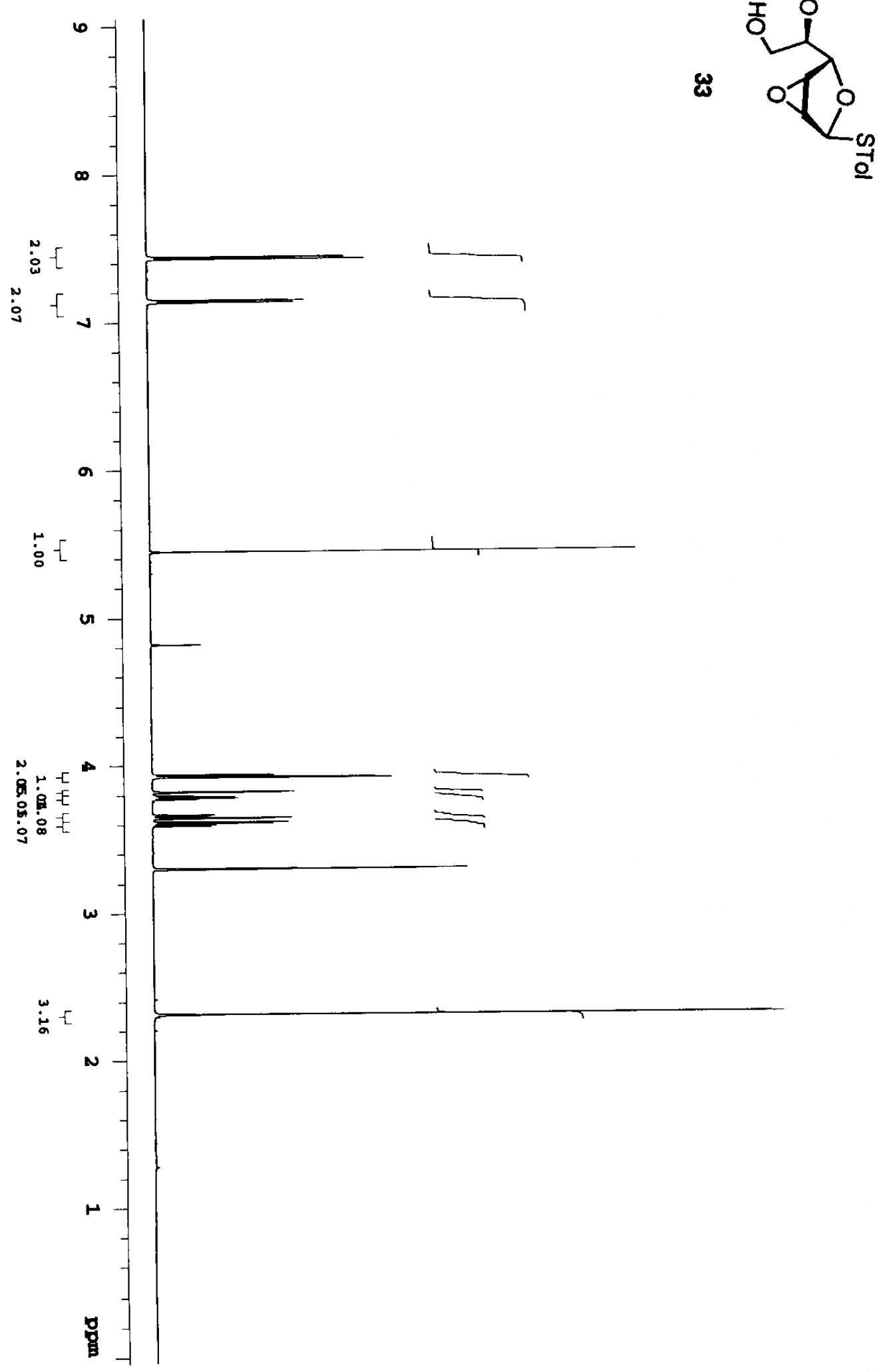

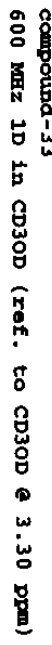



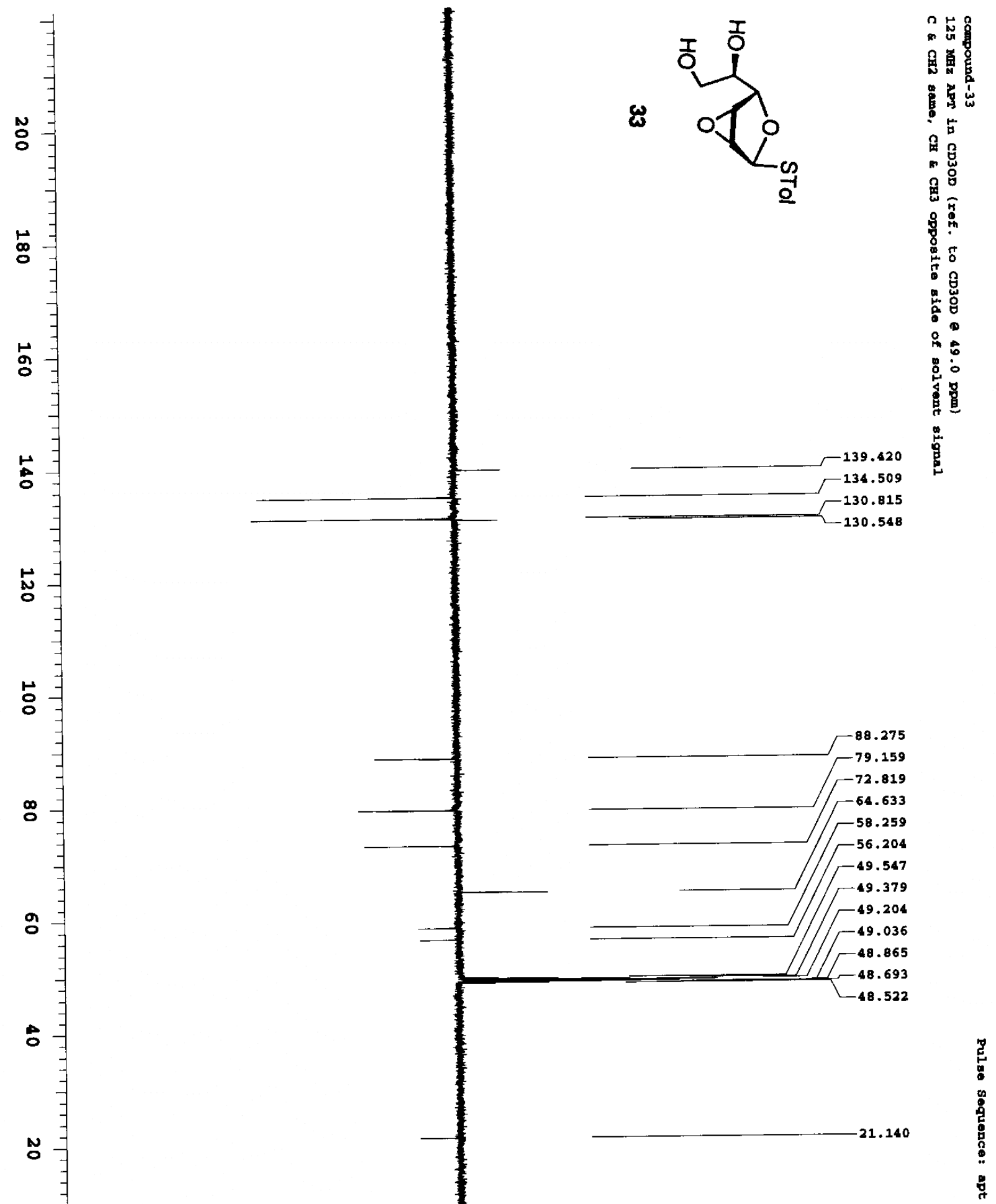

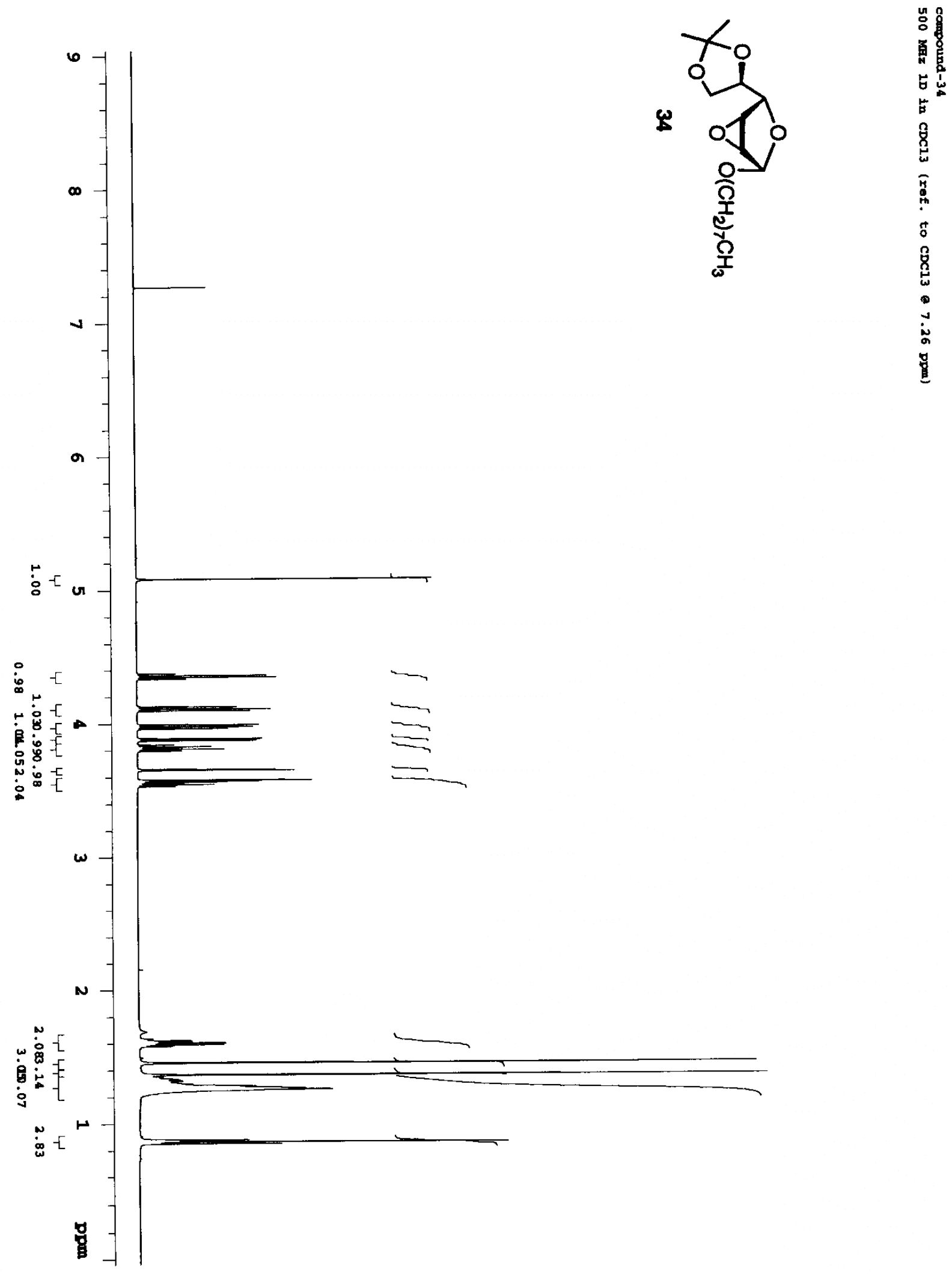

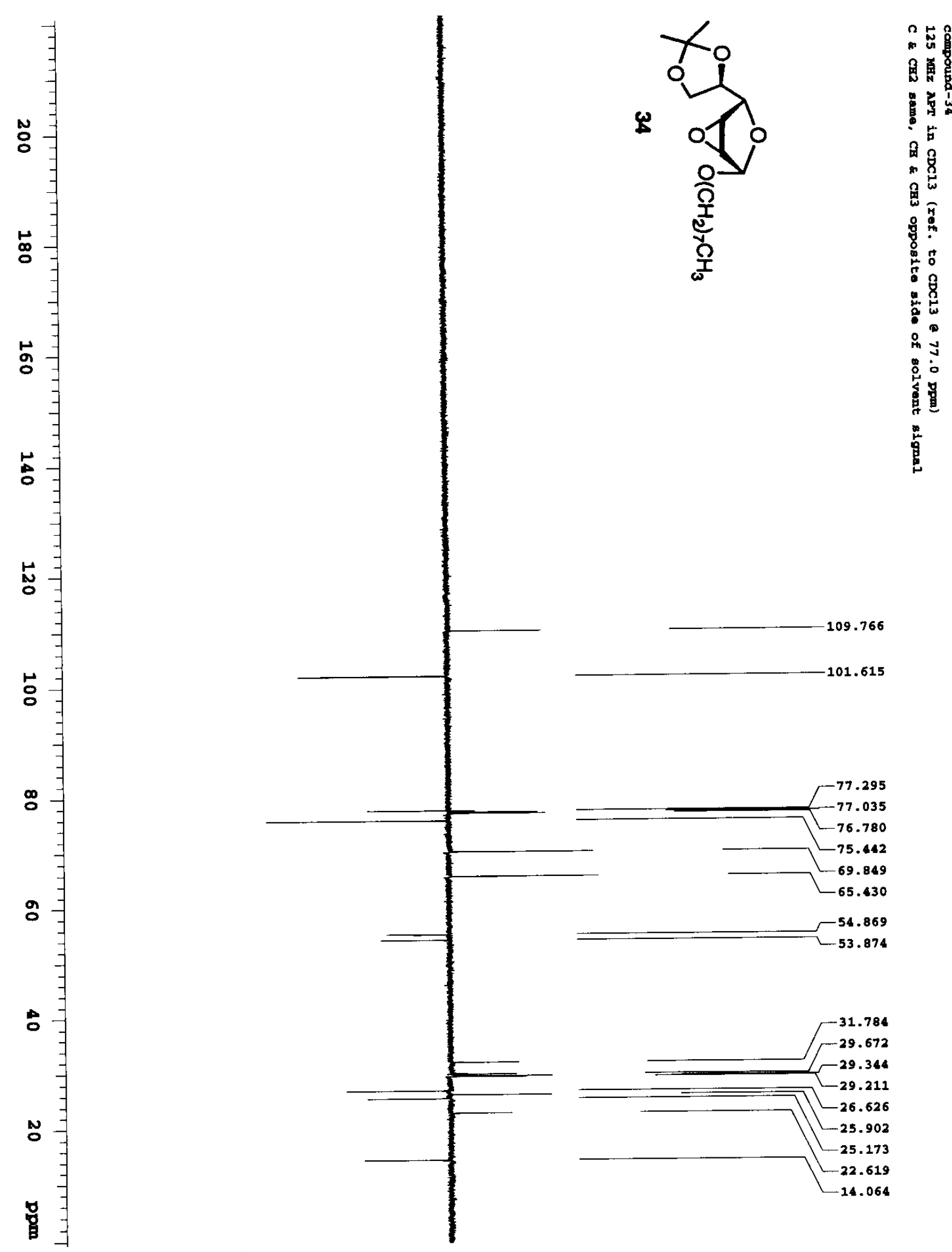


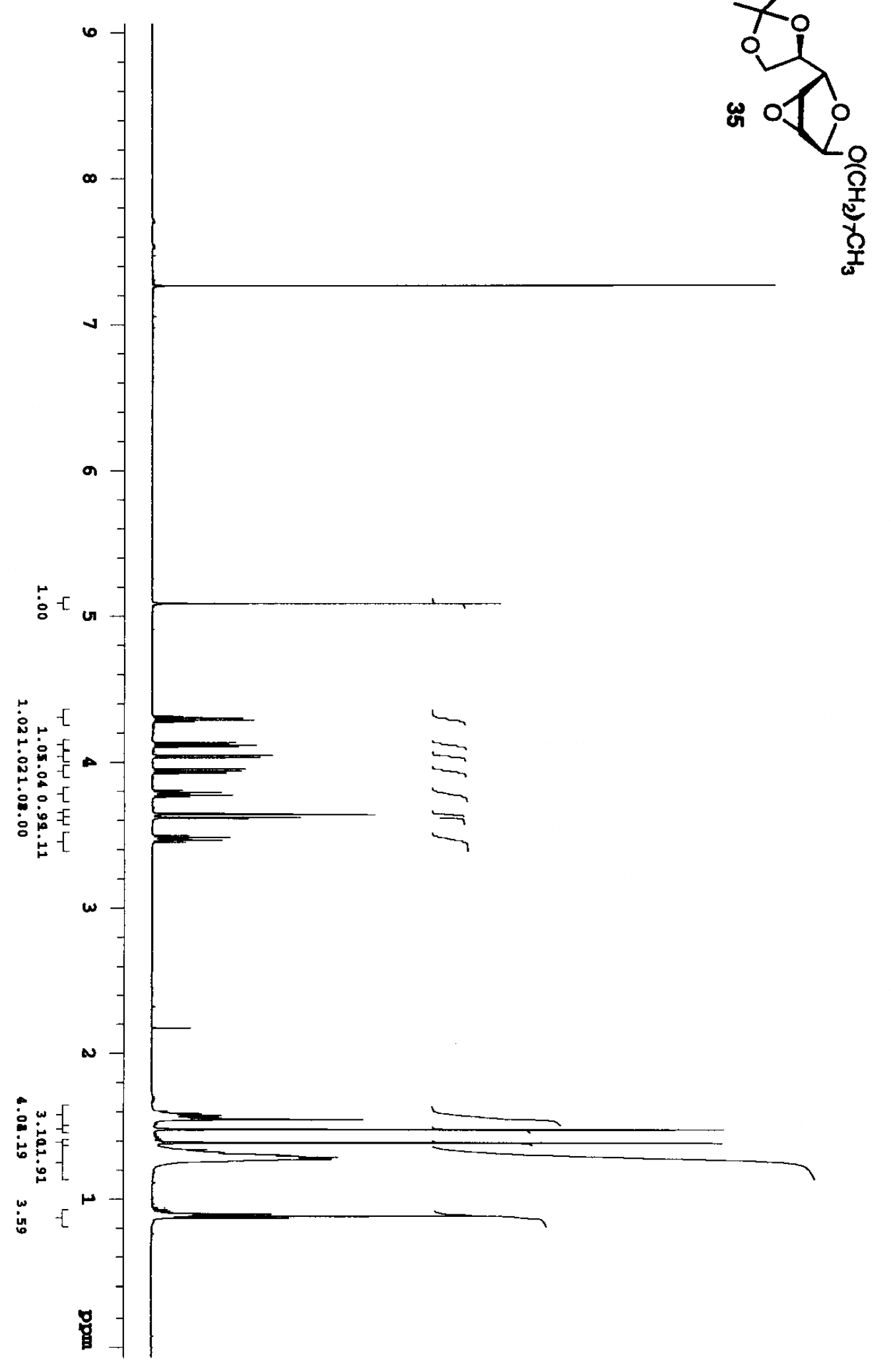

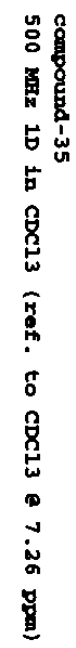

: 

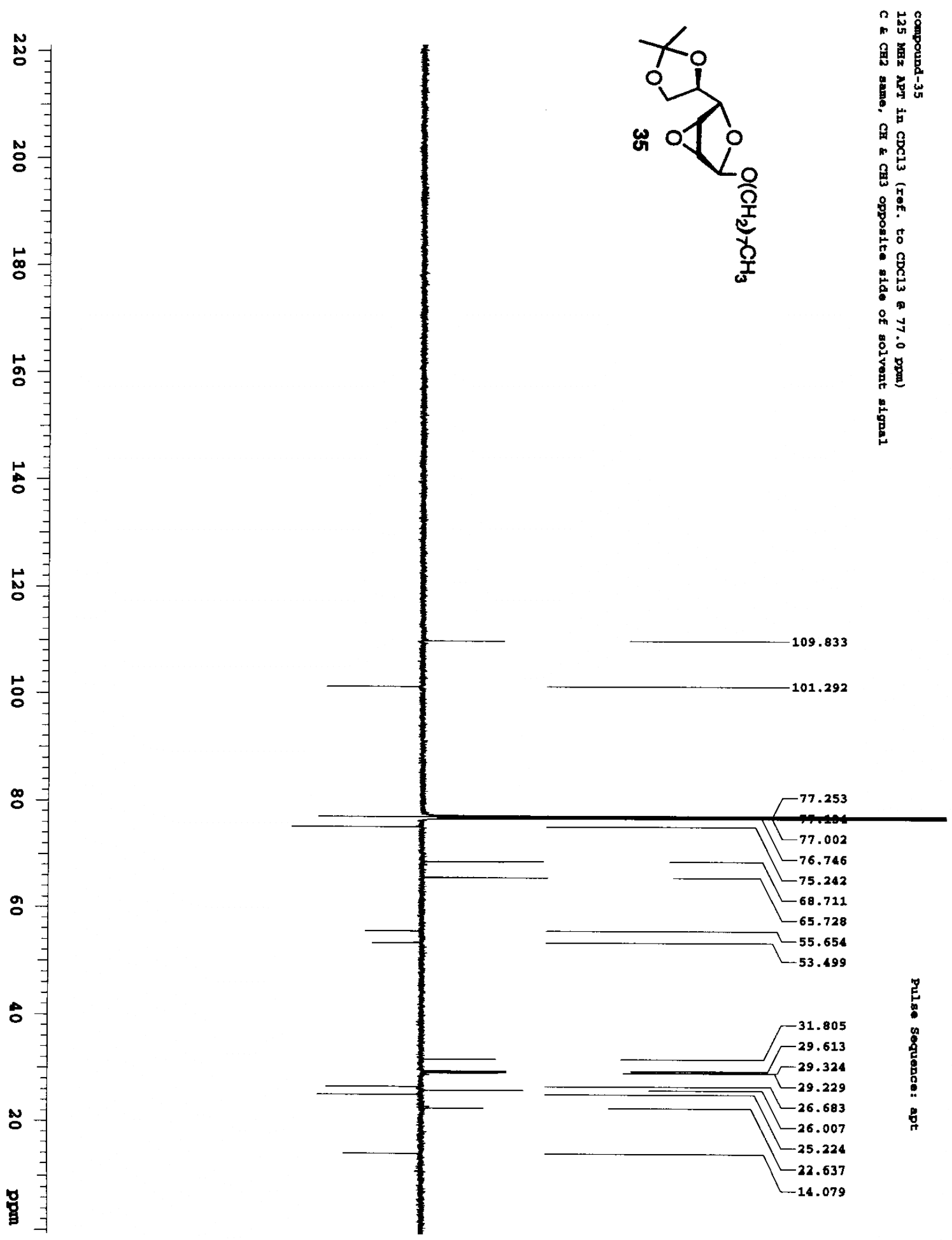

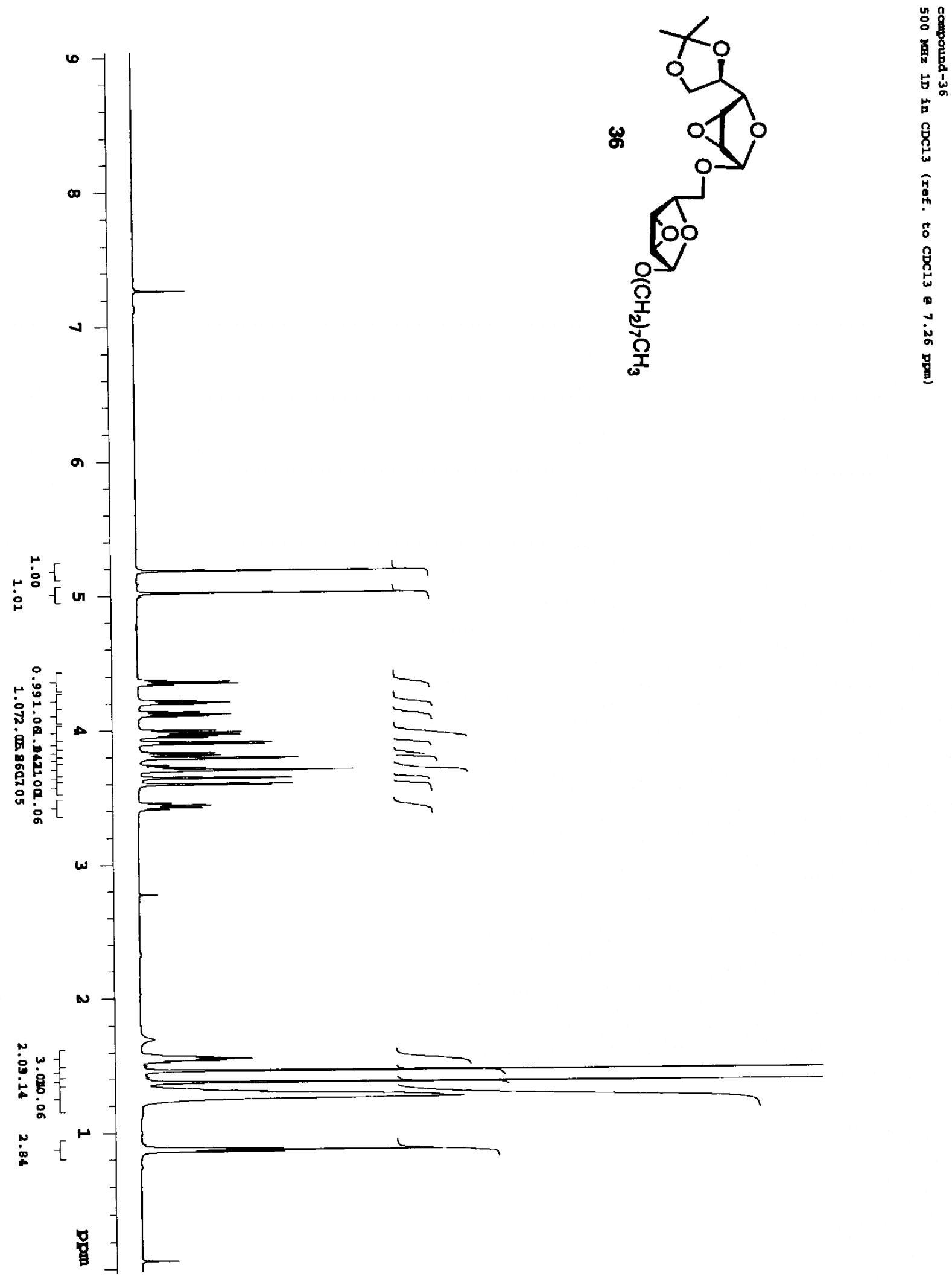

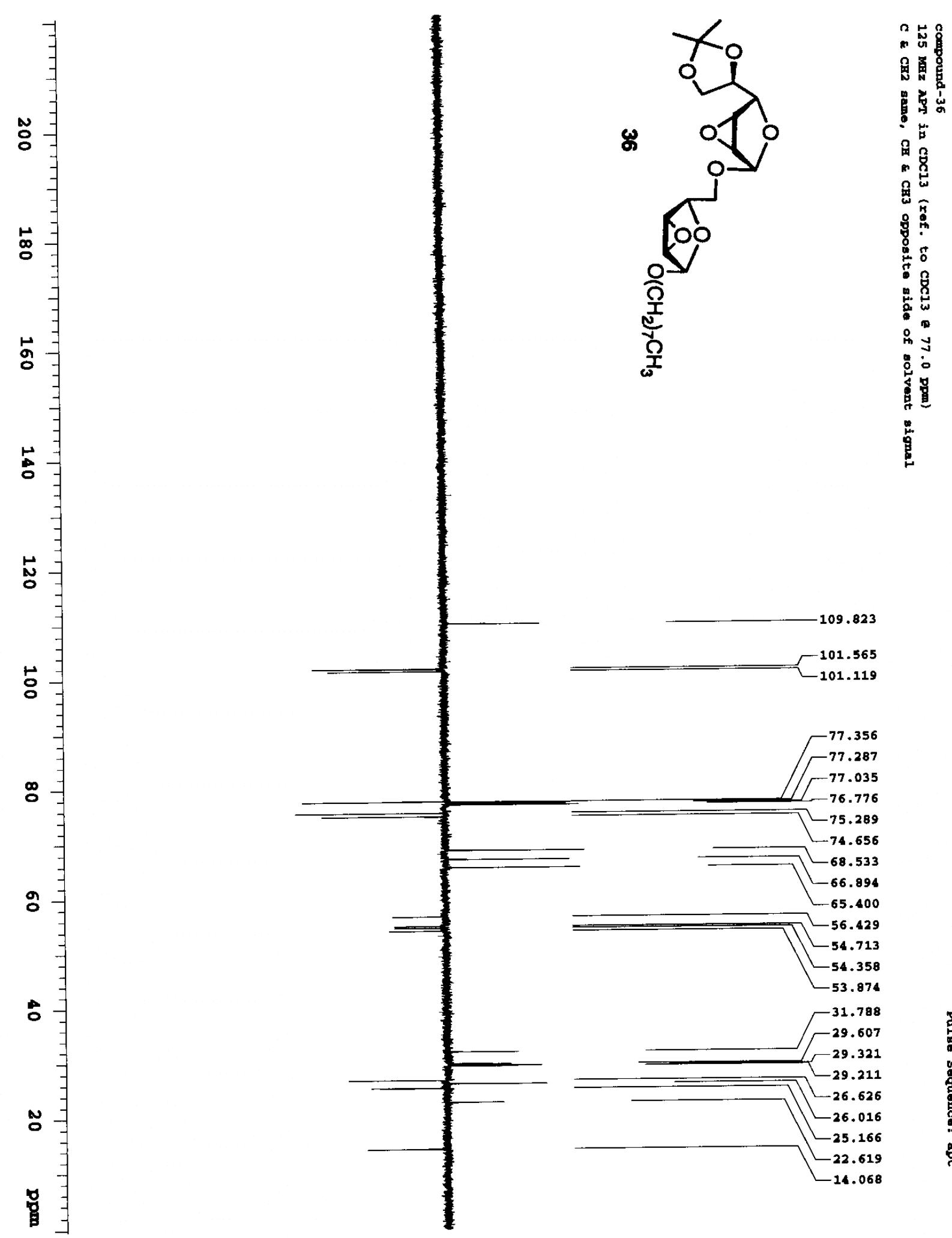


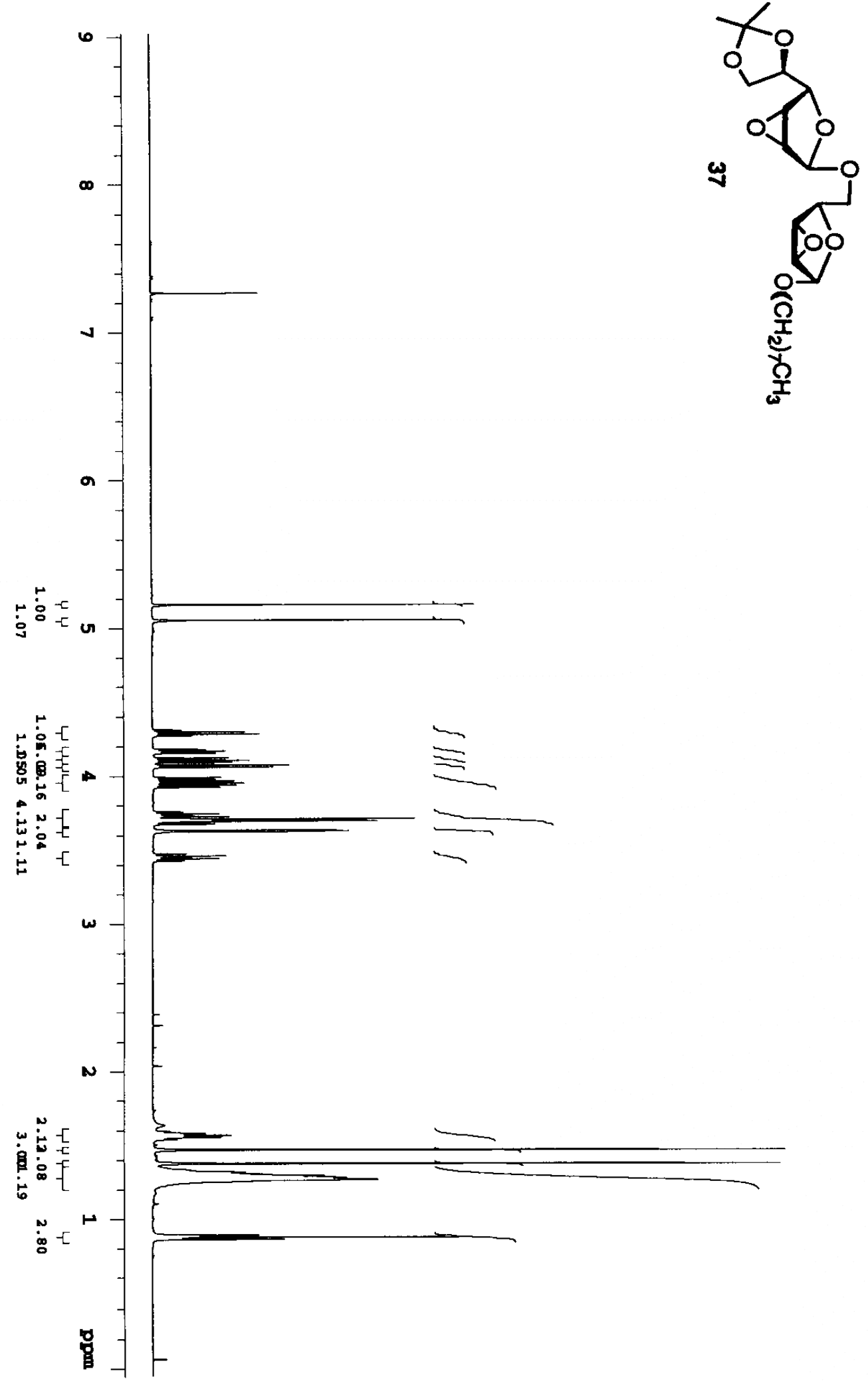

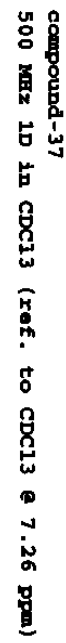




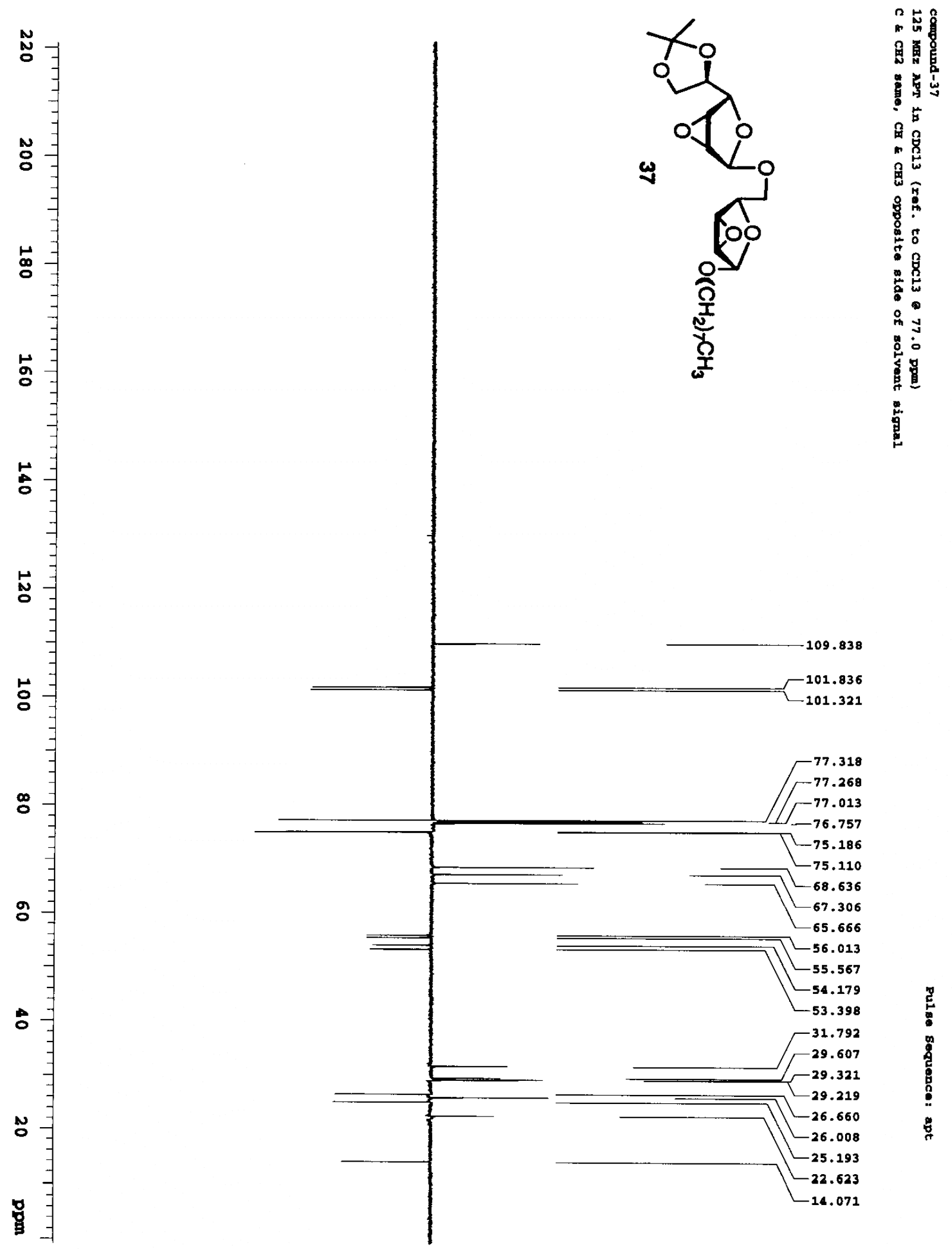



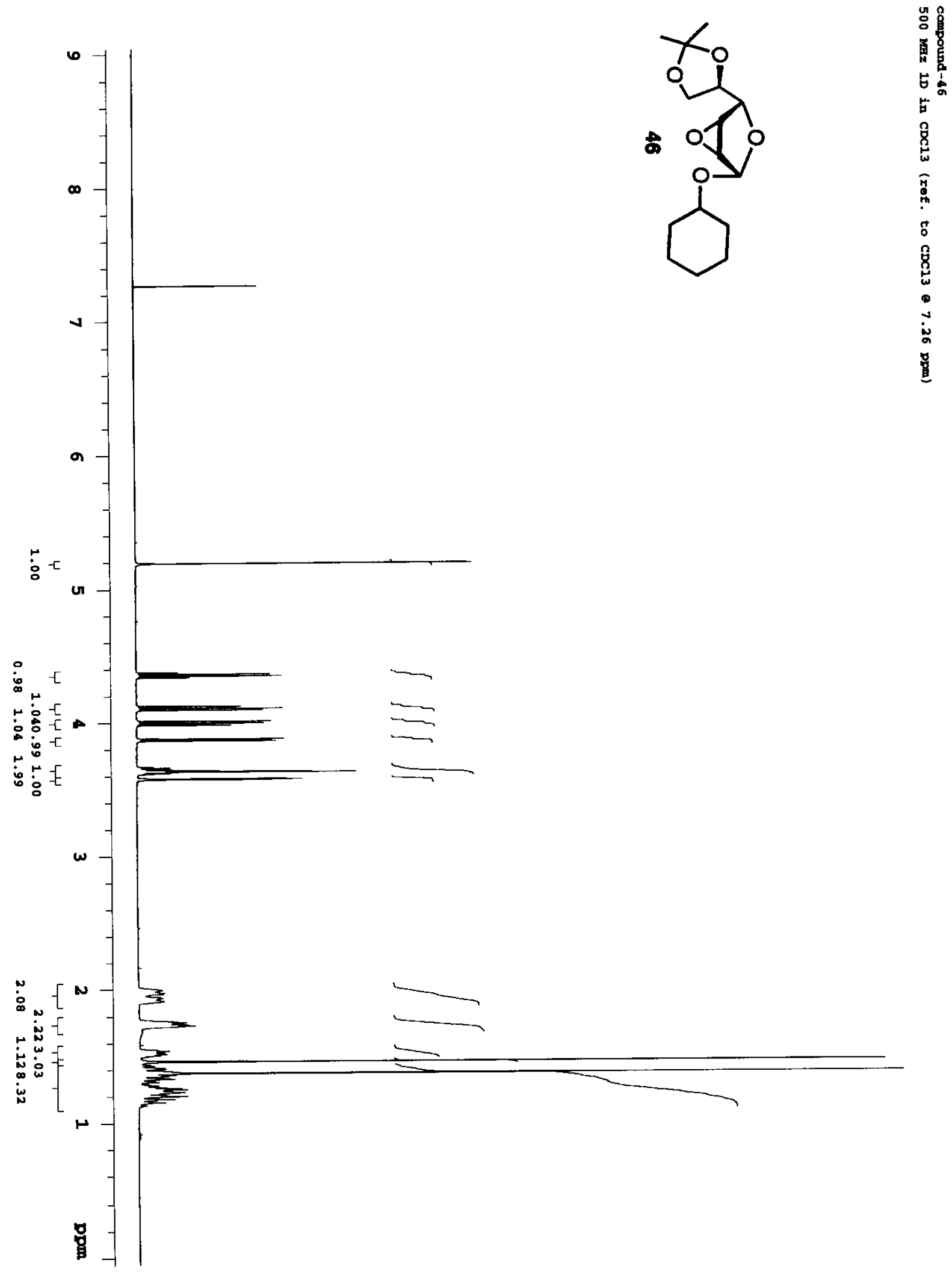


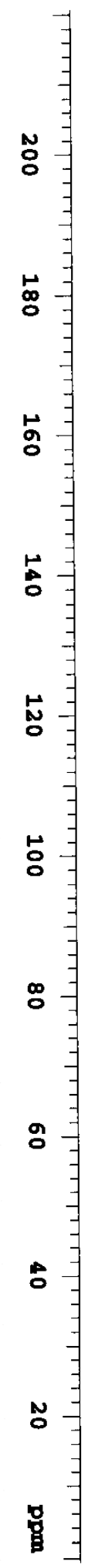

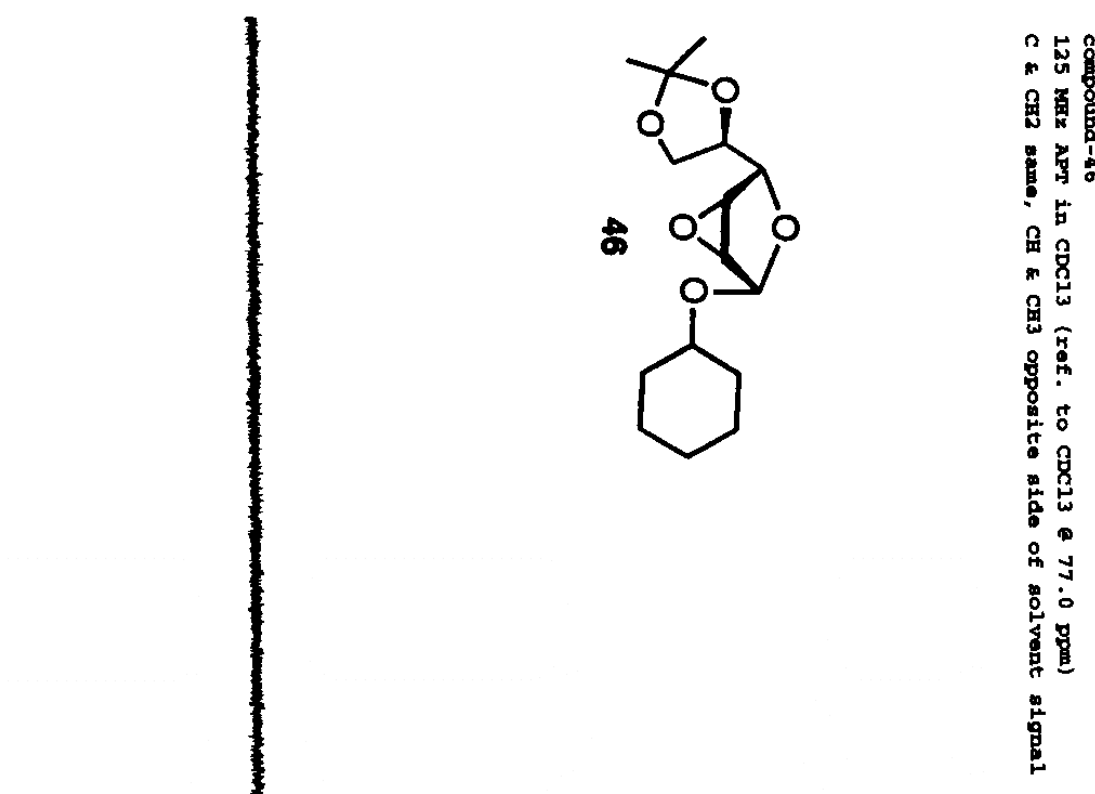




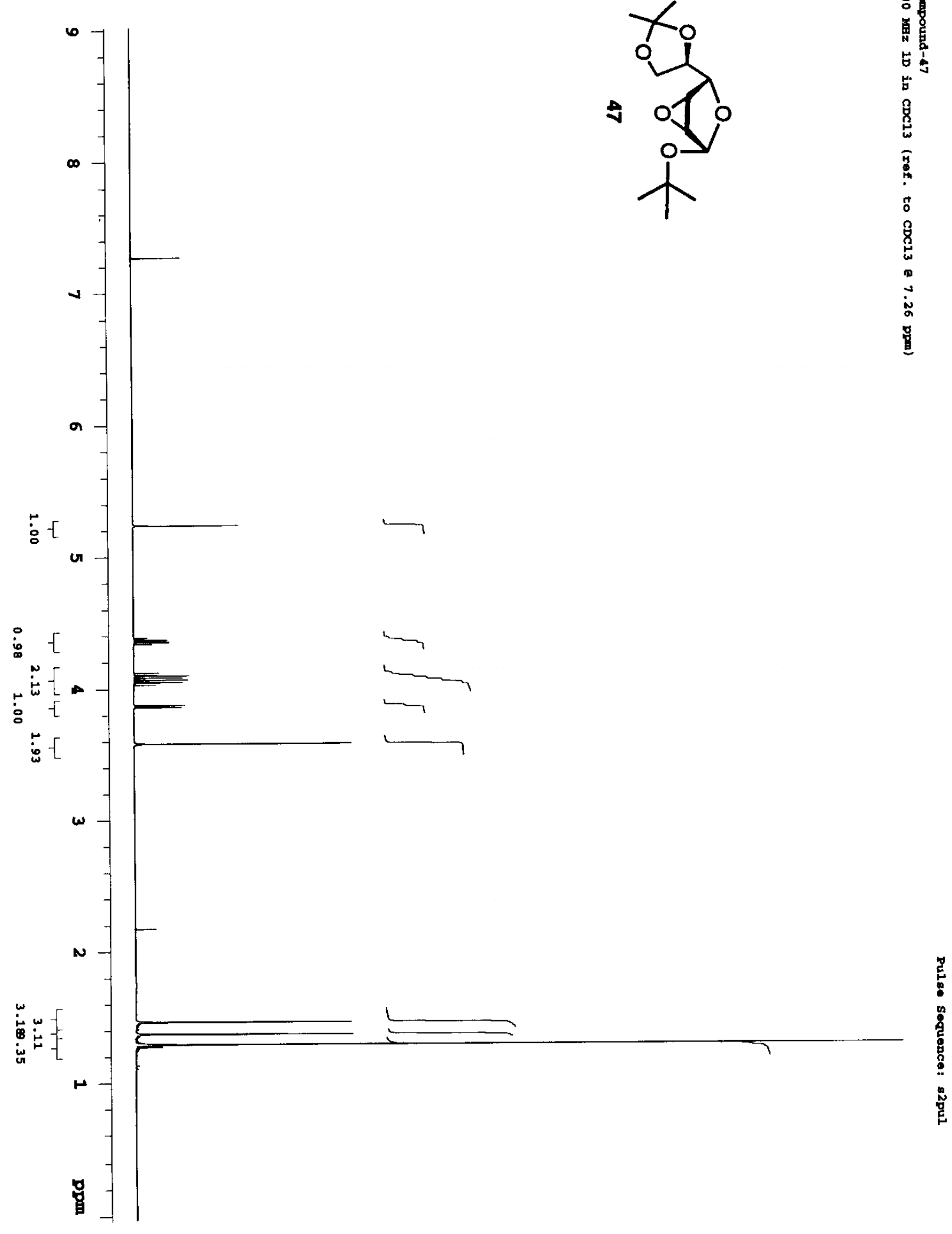




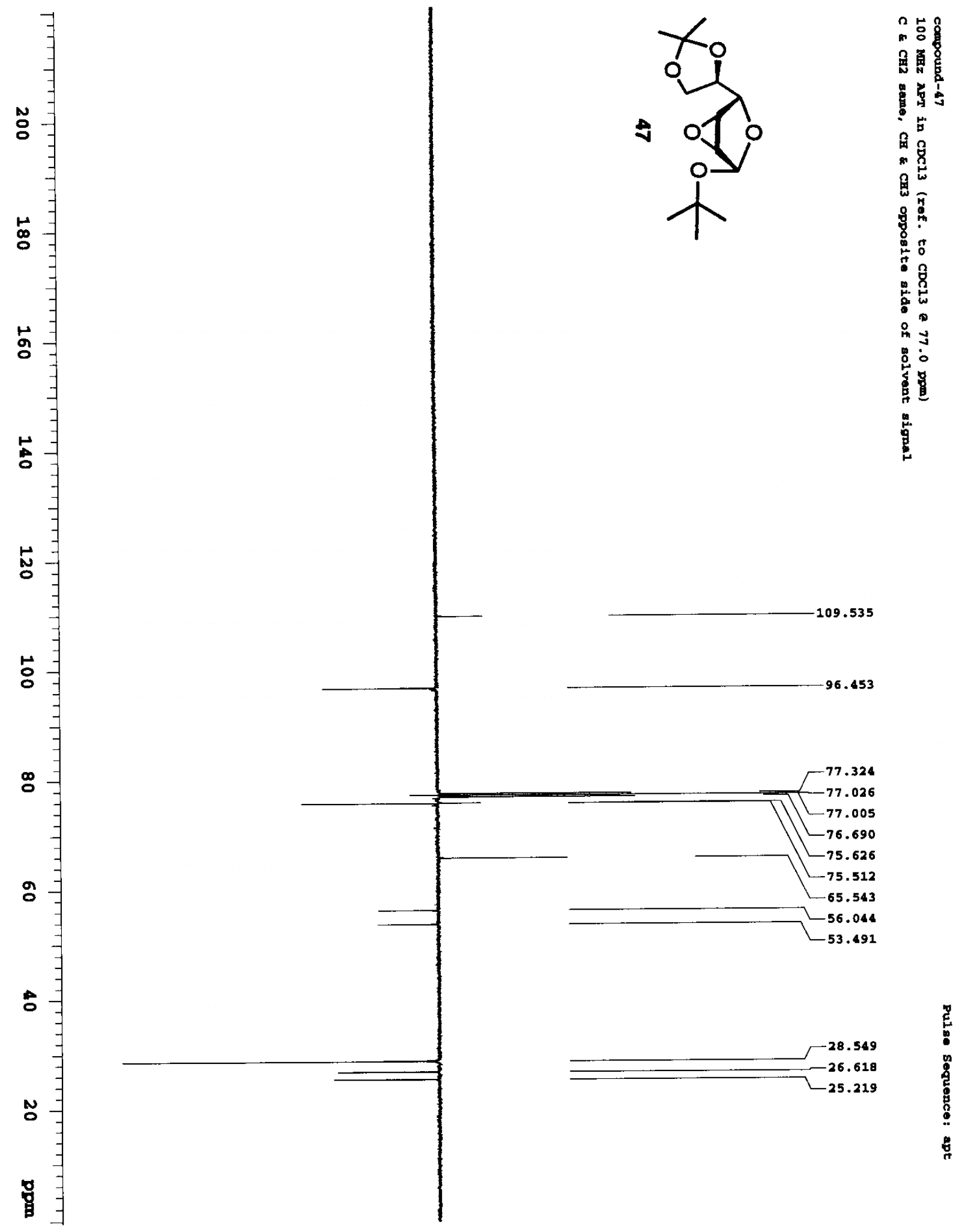




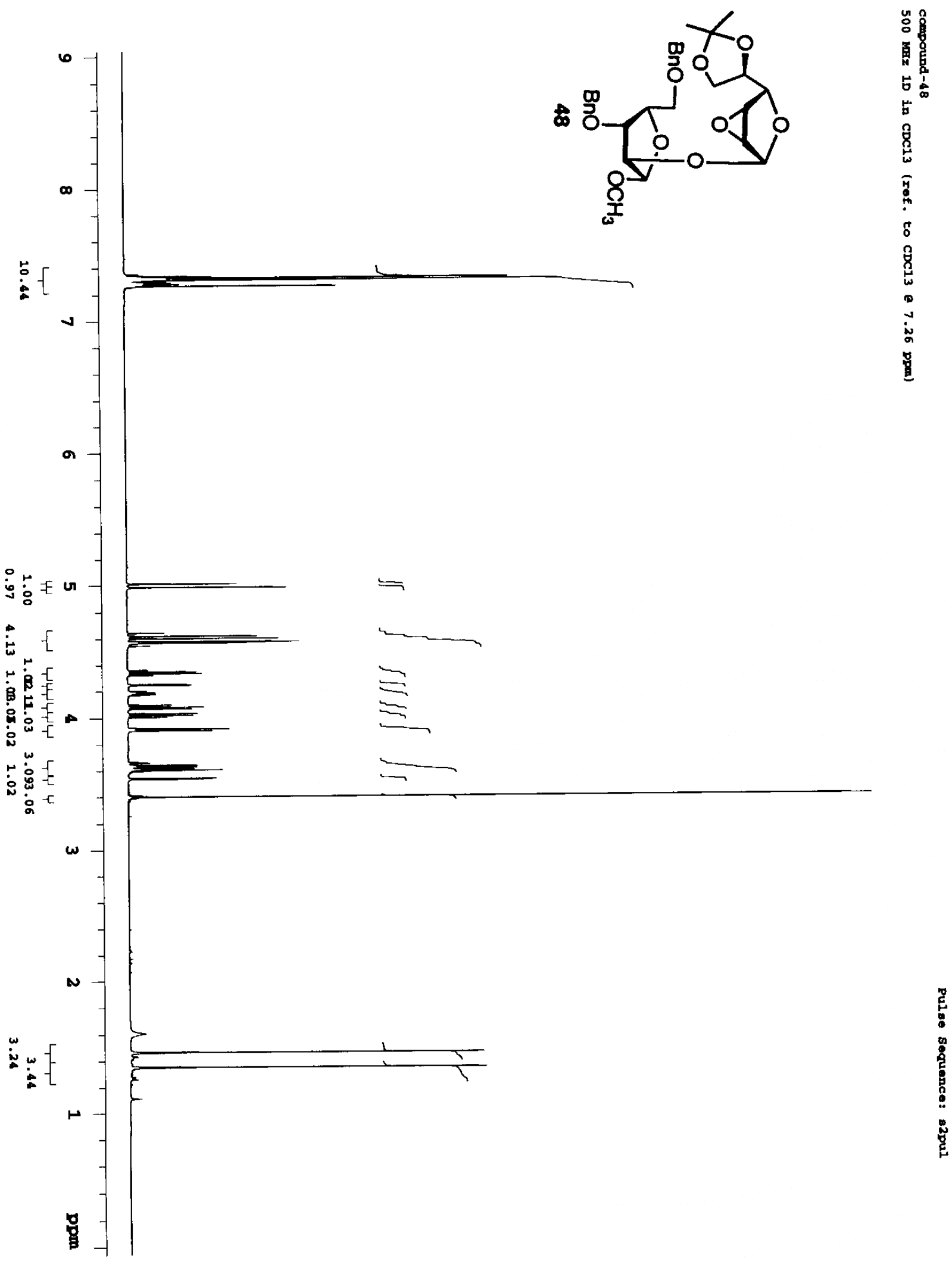




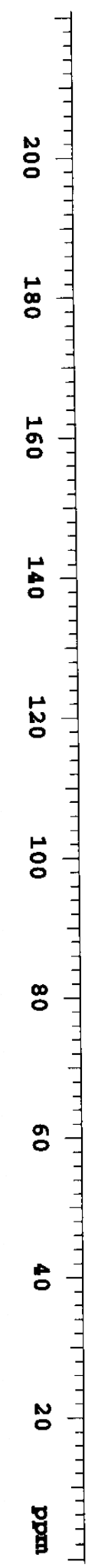

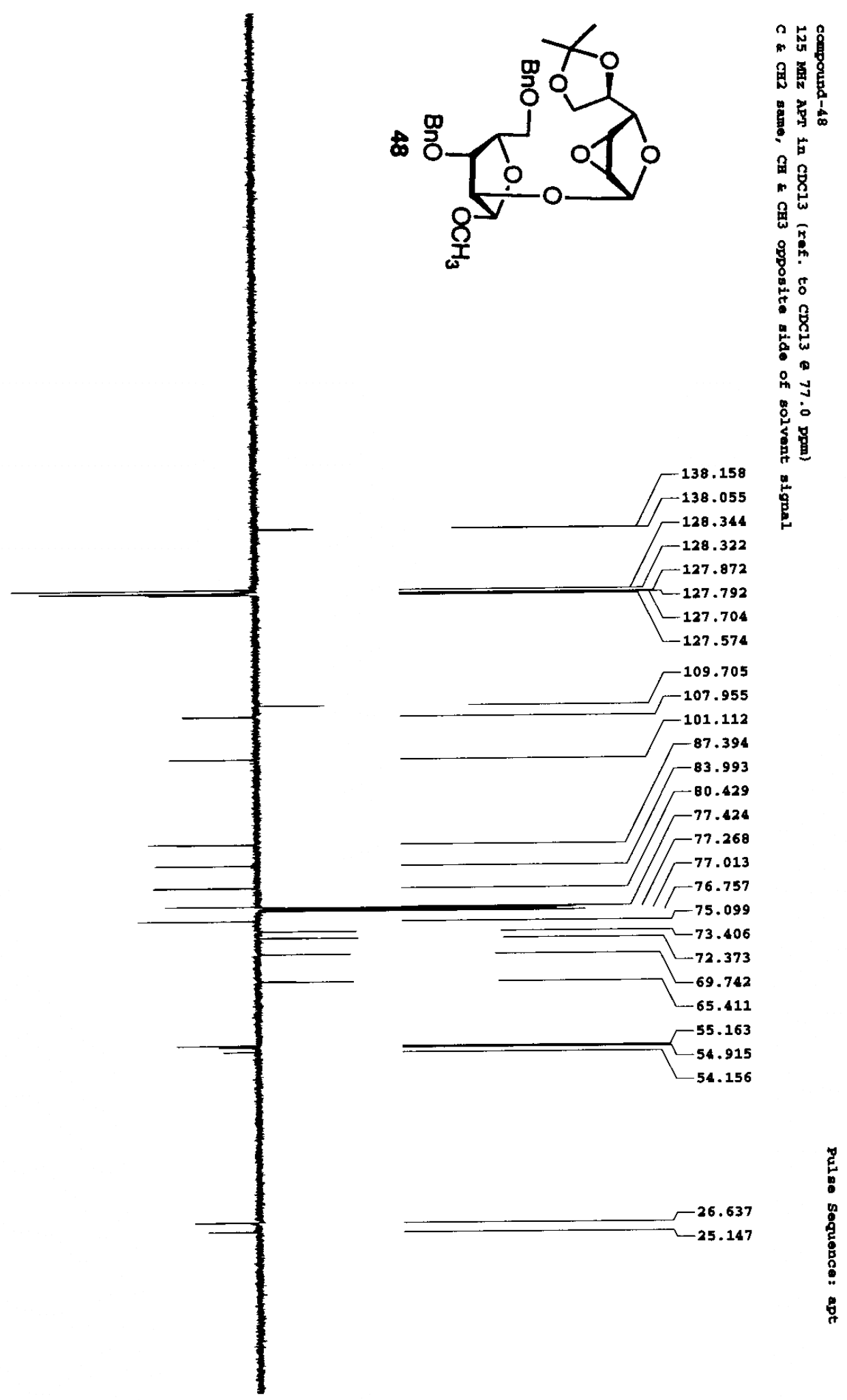




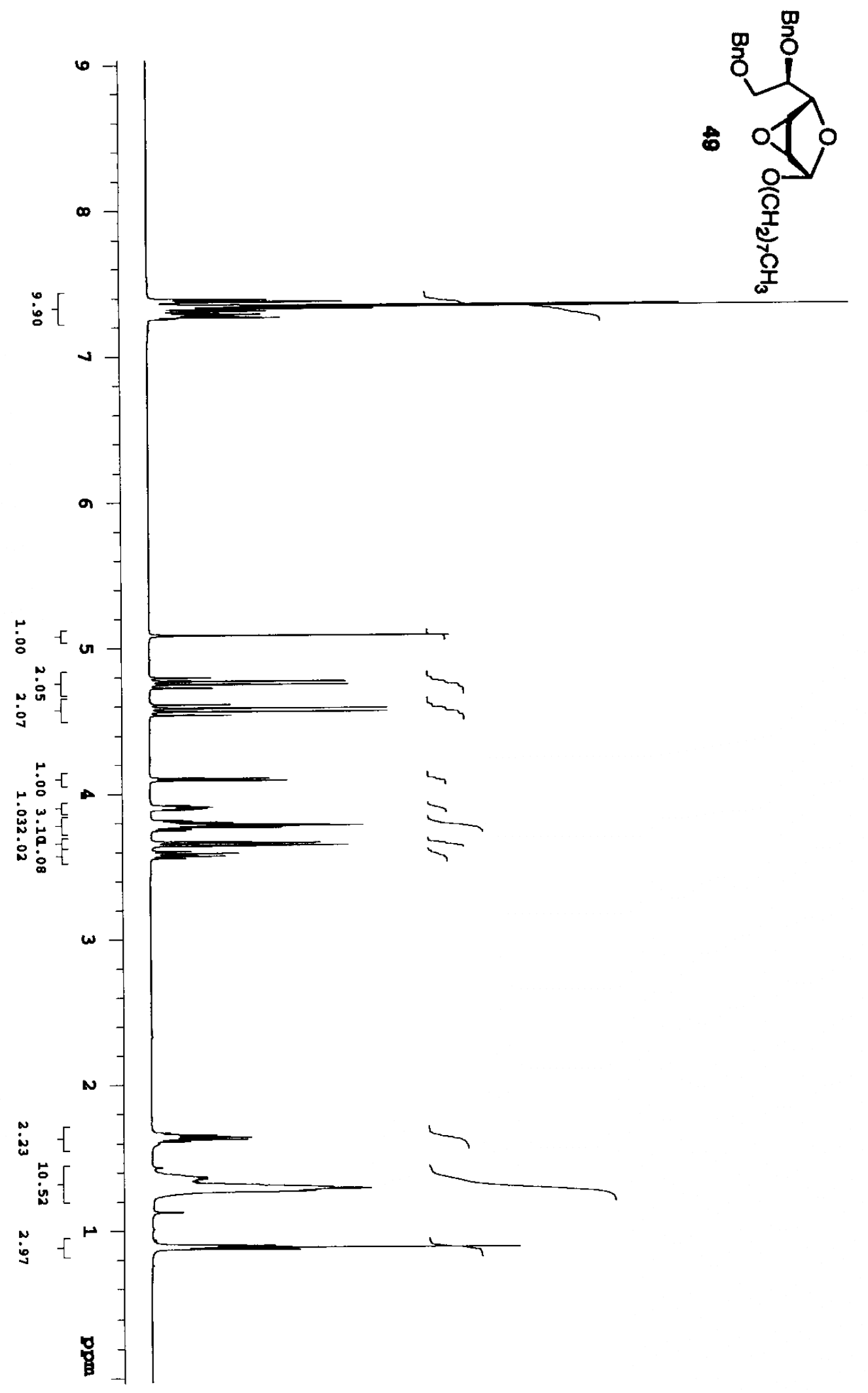

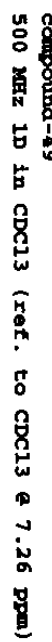



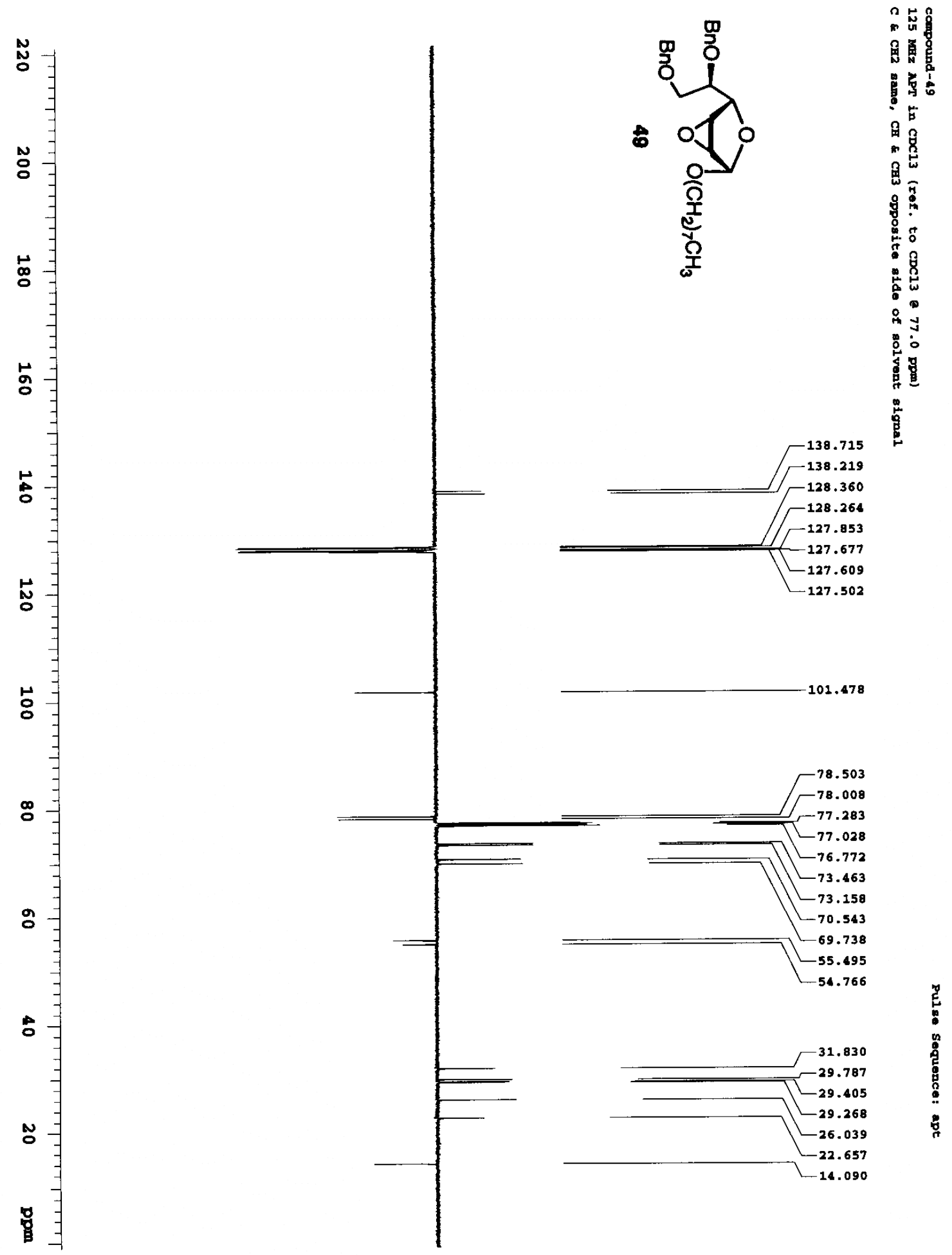


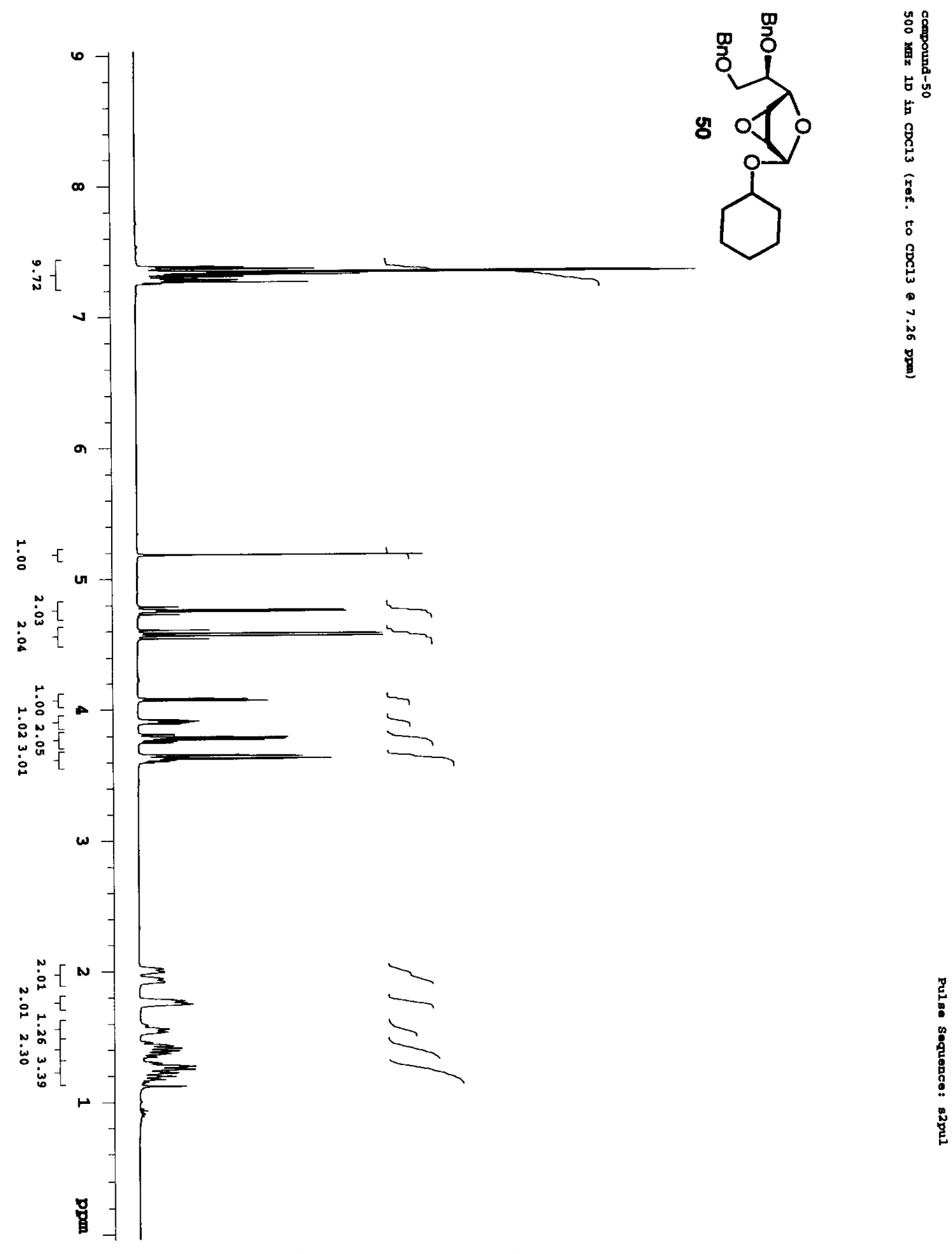




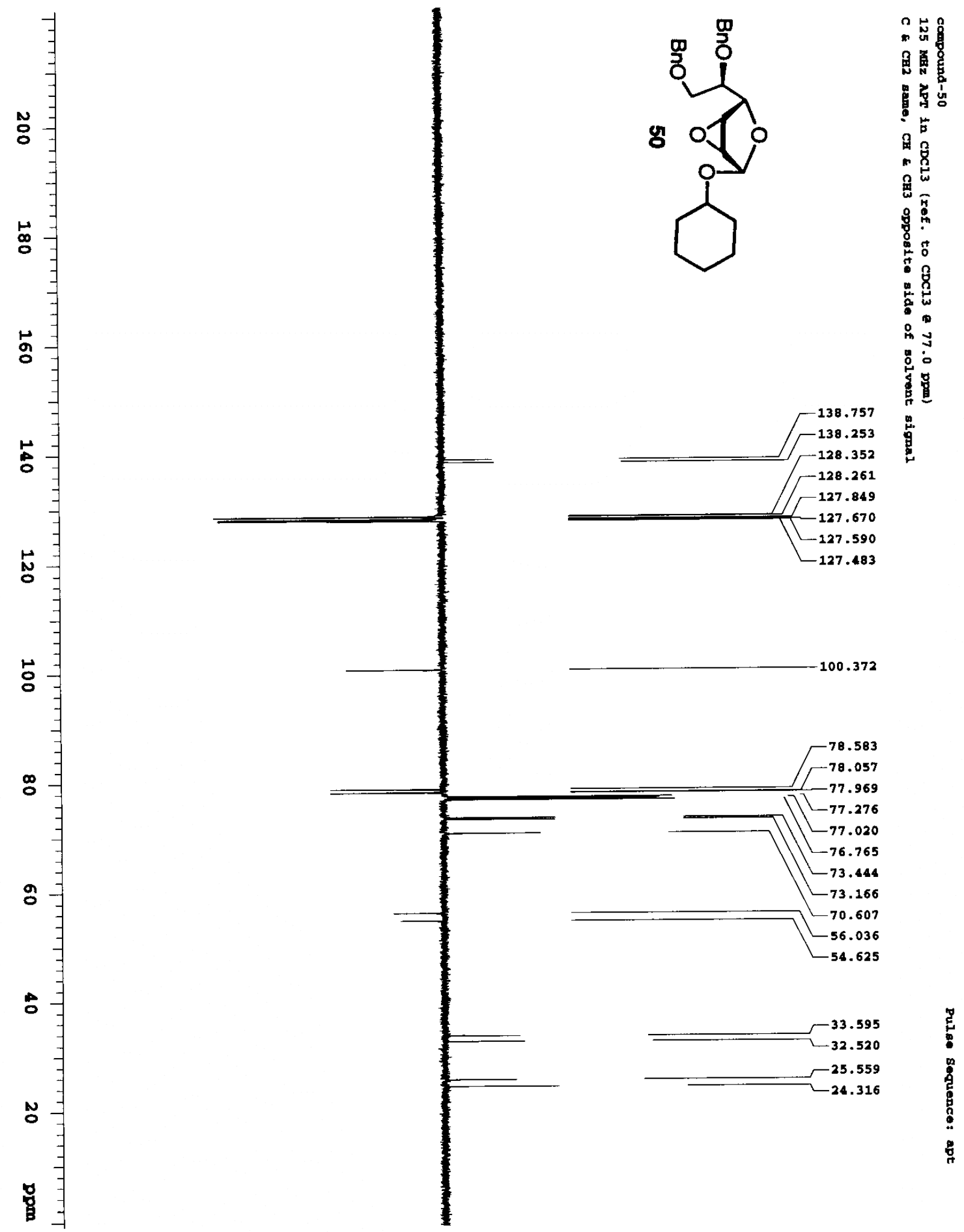




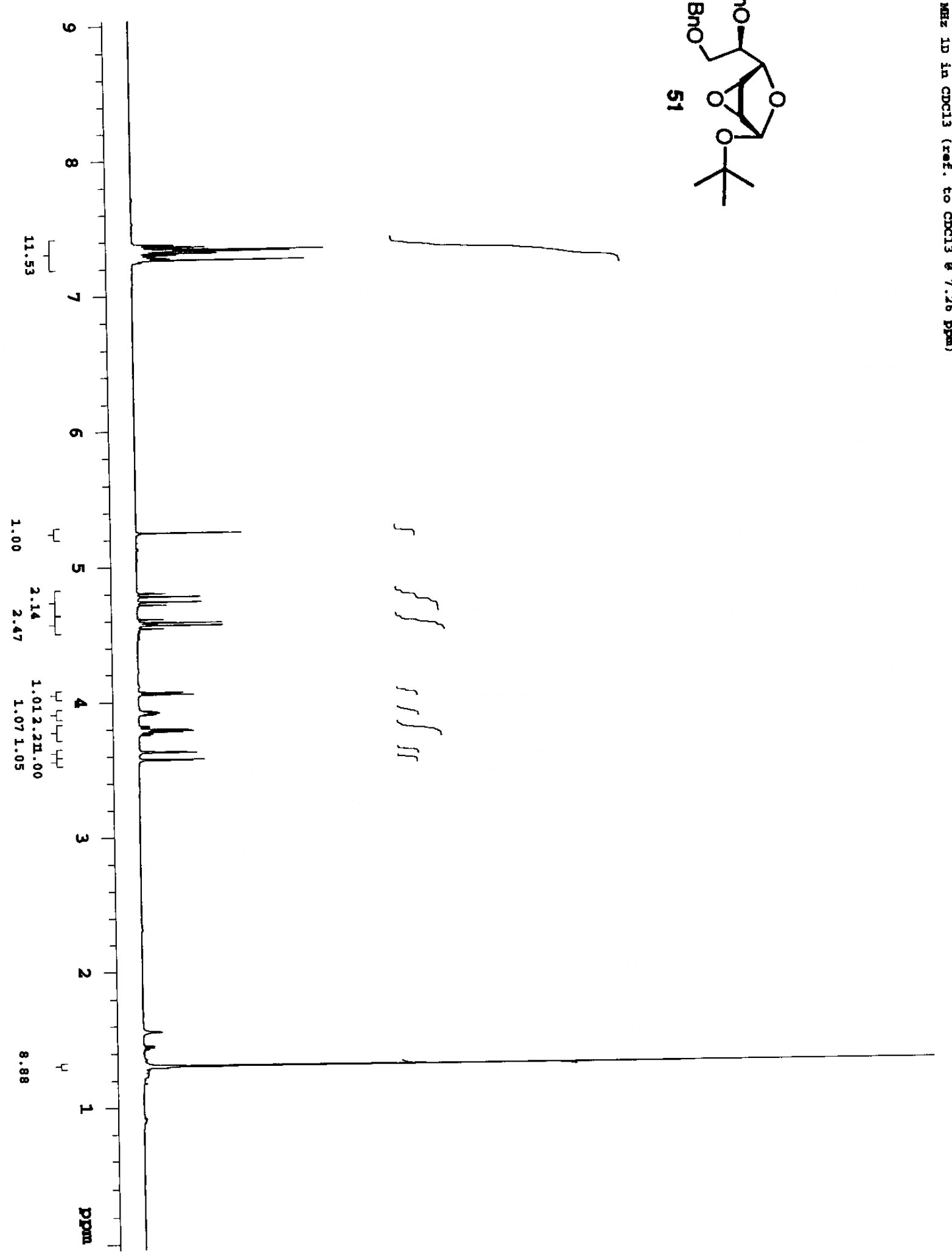




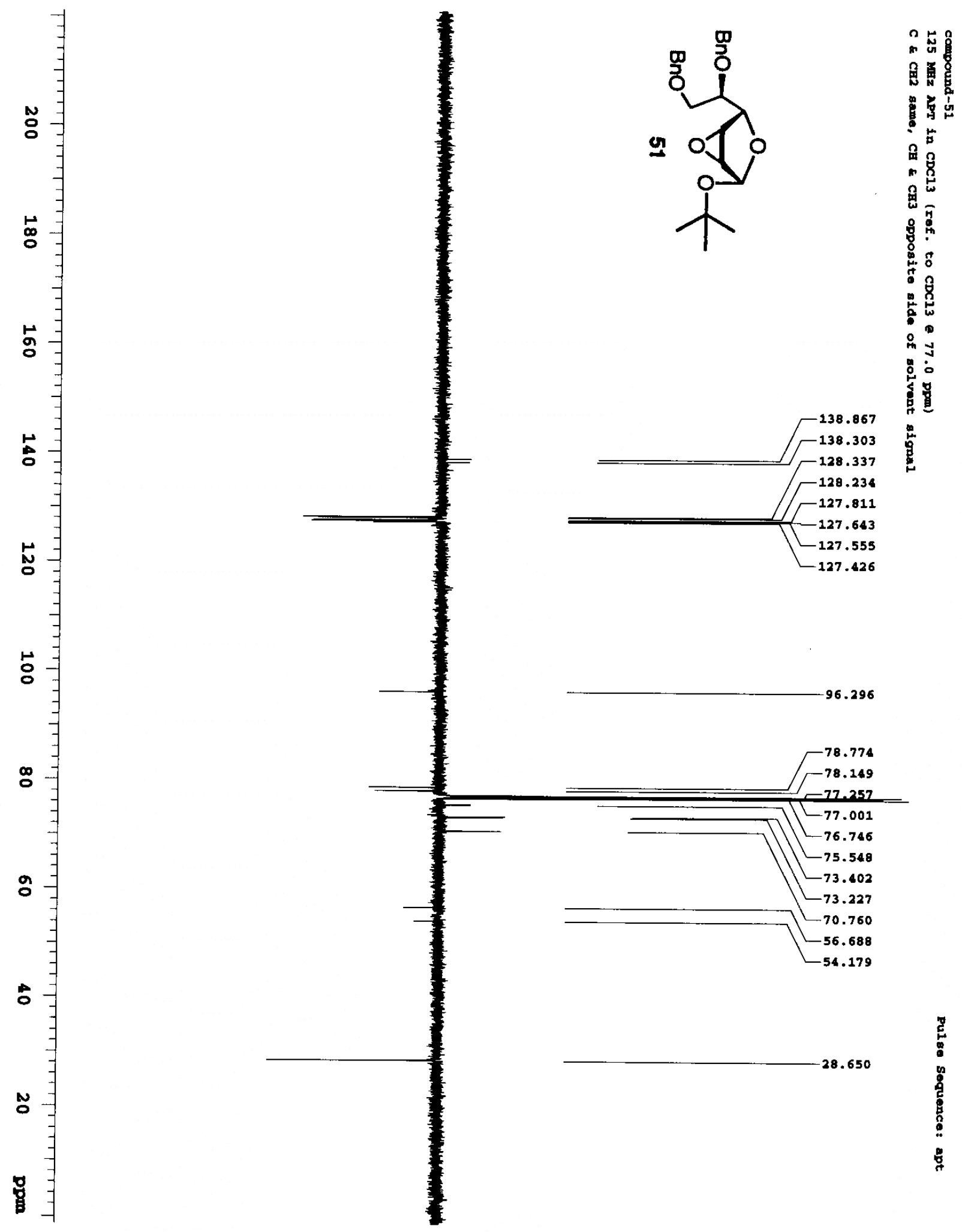



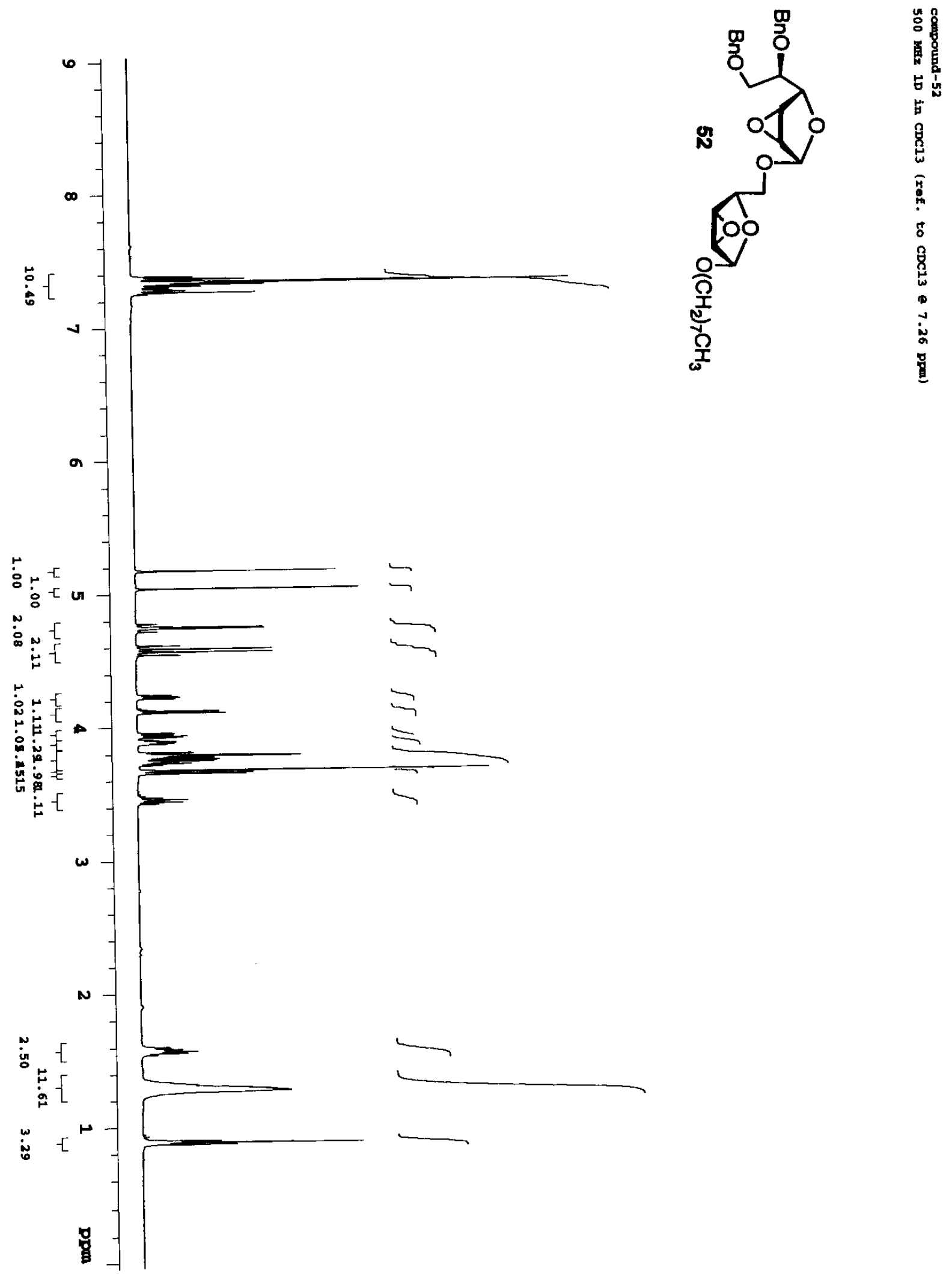

है 


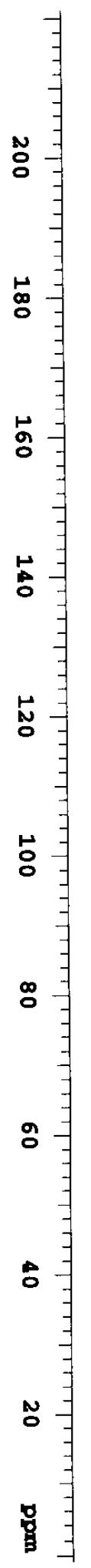

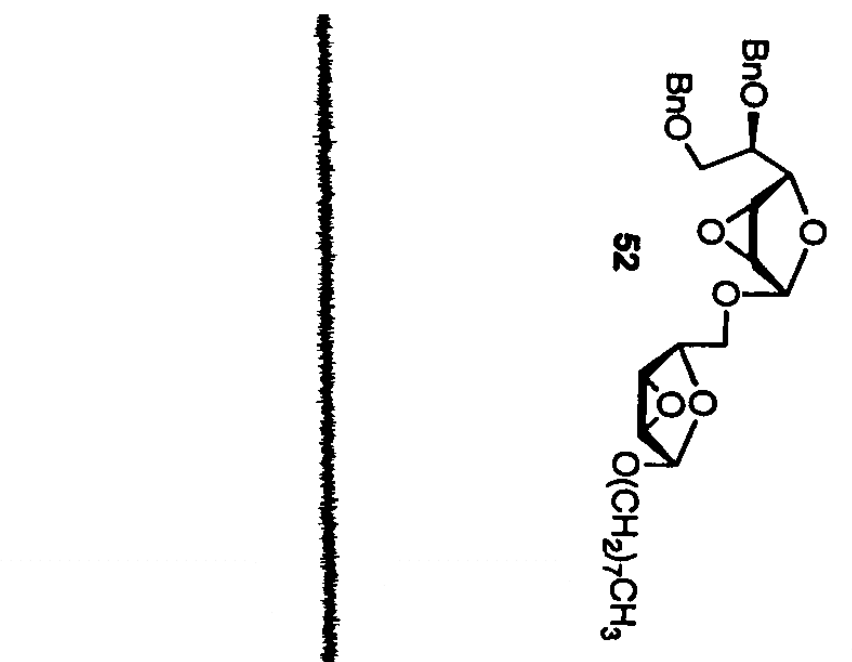

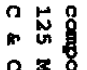

邑急

留

占

뭉

क

思

8.

क व

8

总点

잉

is:

案
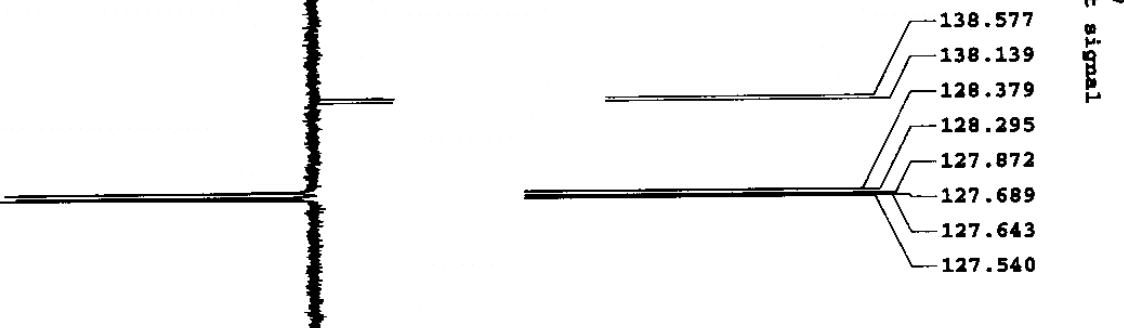

$-101.500$

78.328
-78.126
-77.276

${ }^{77.024}$

$-76.768$
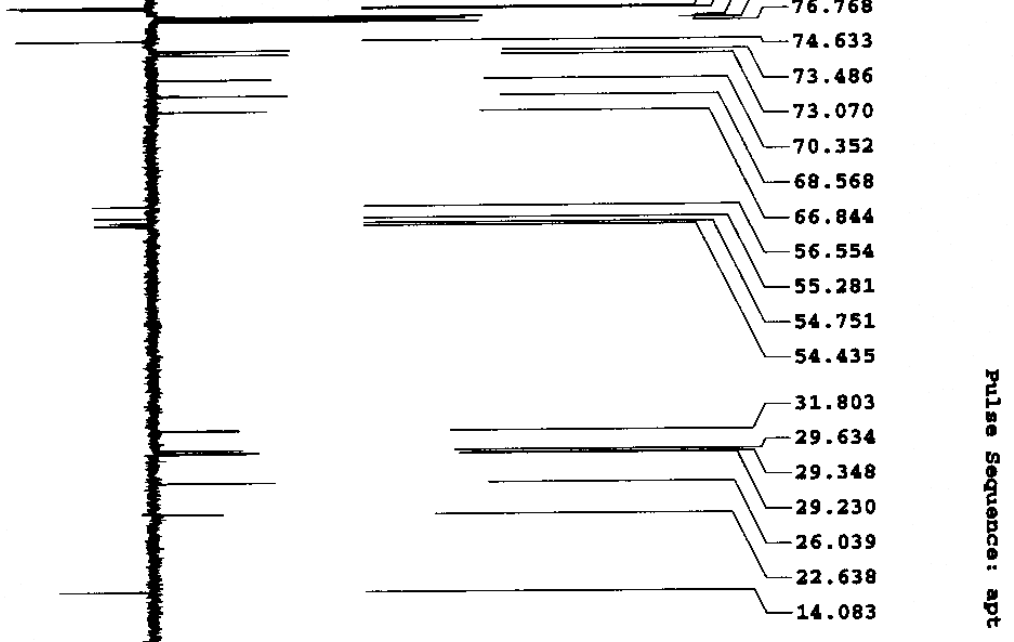


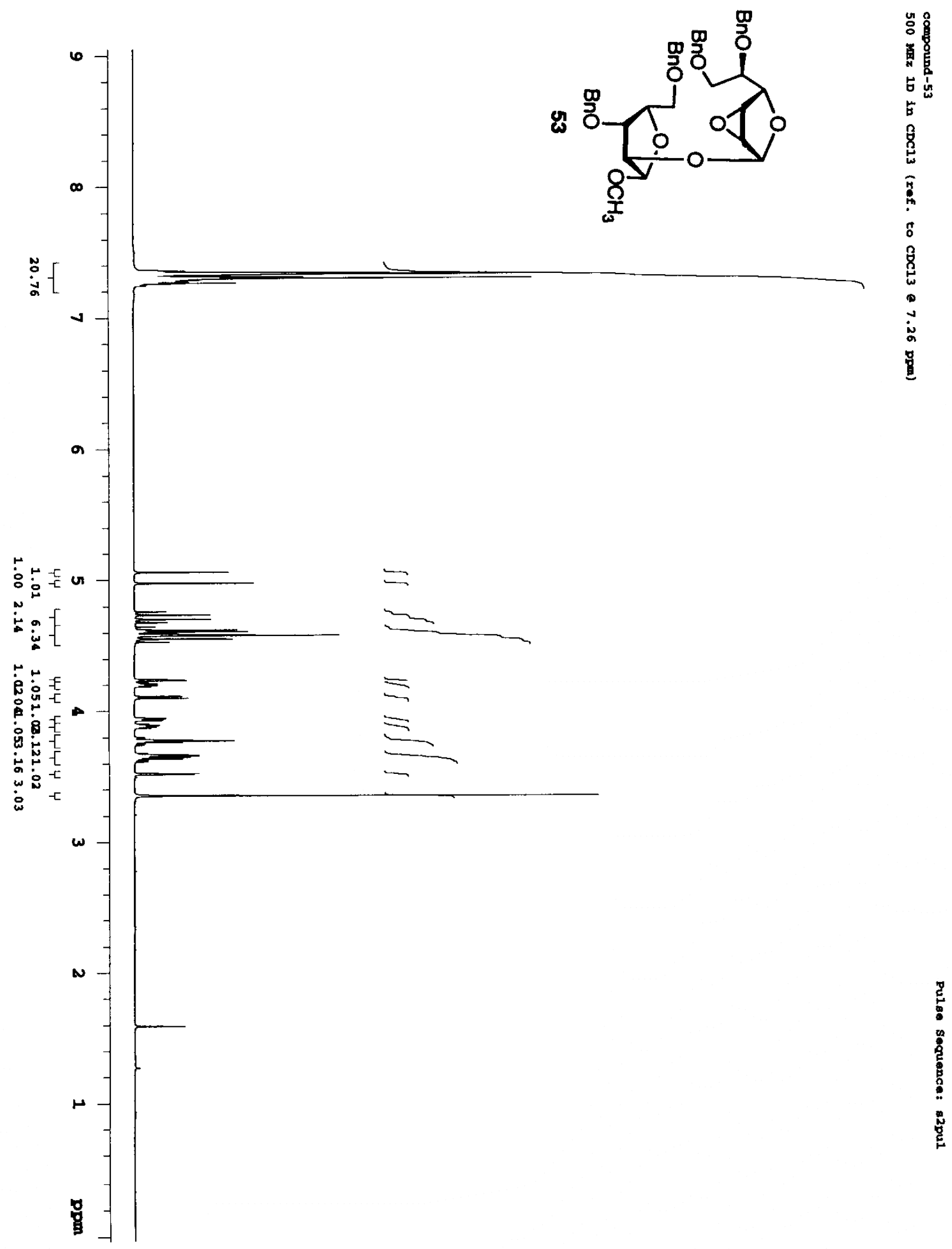




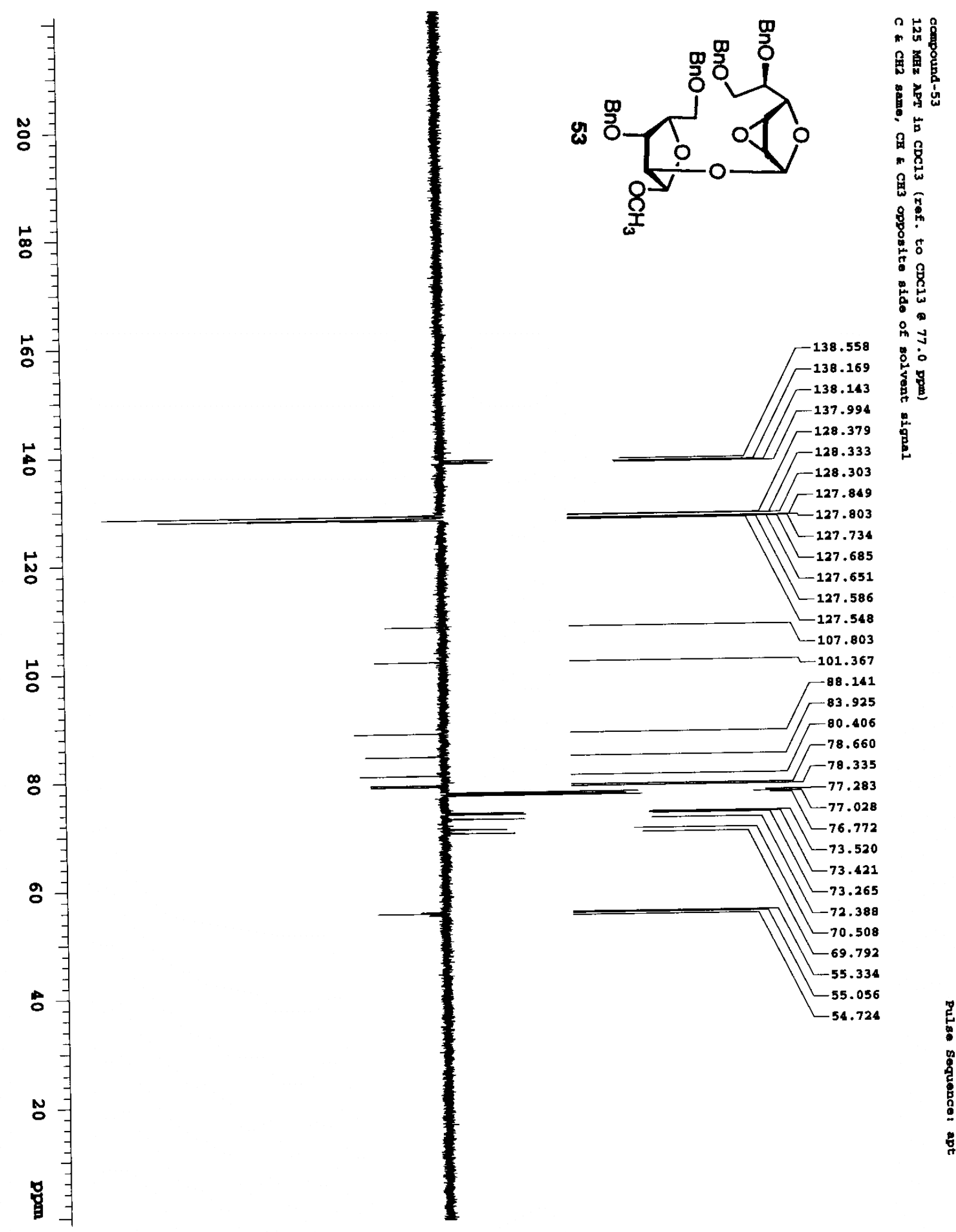



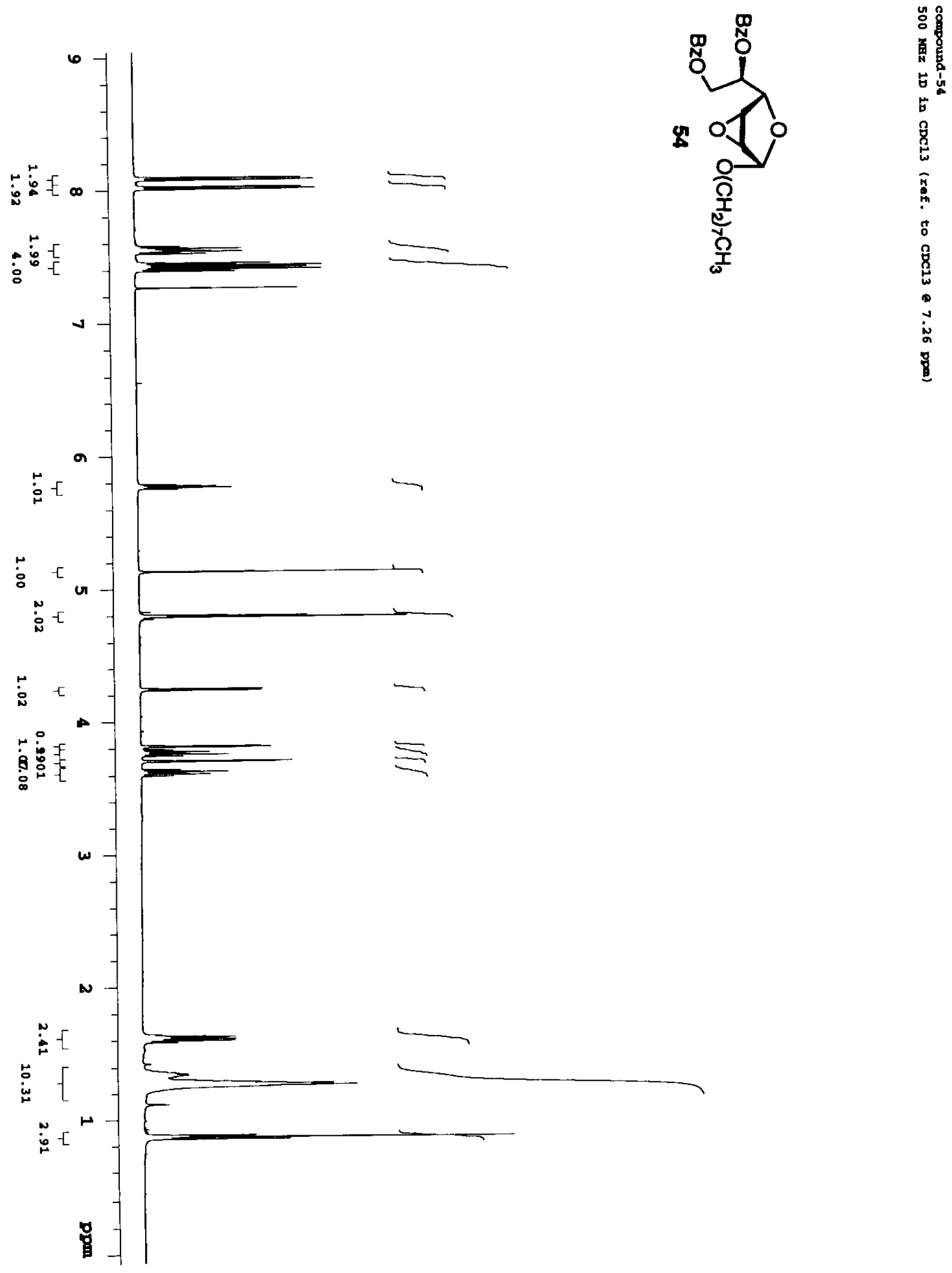


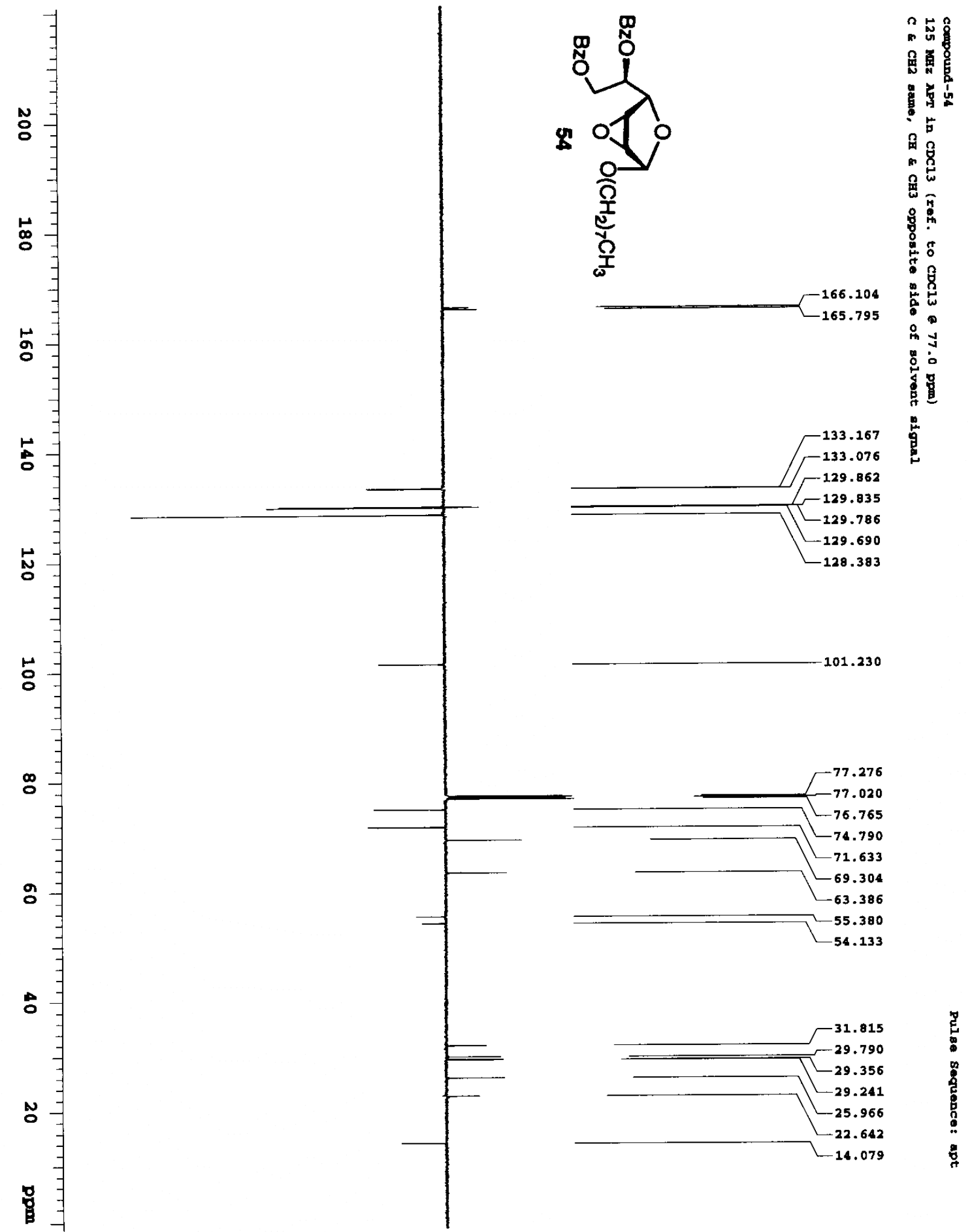



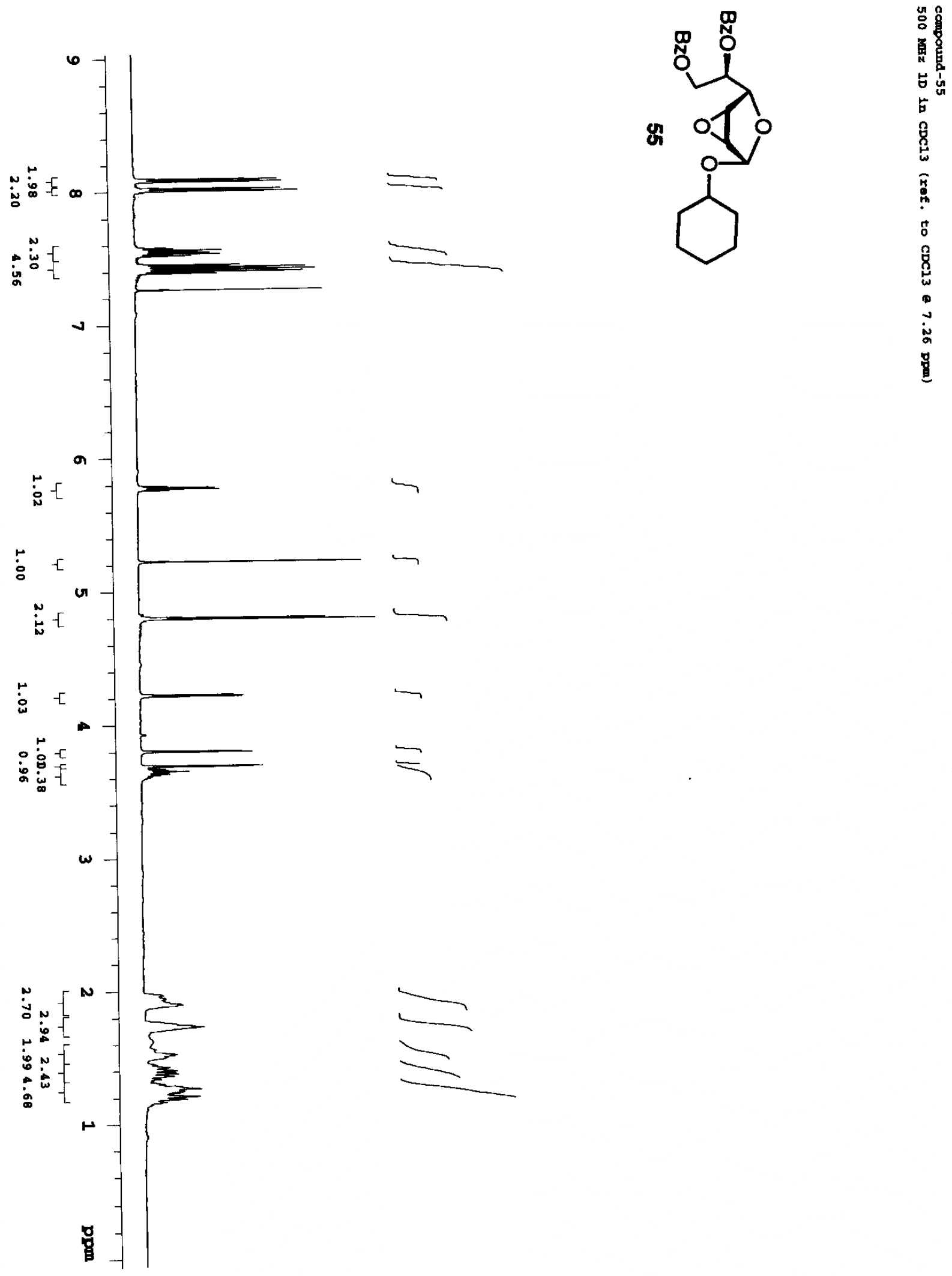

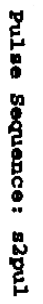




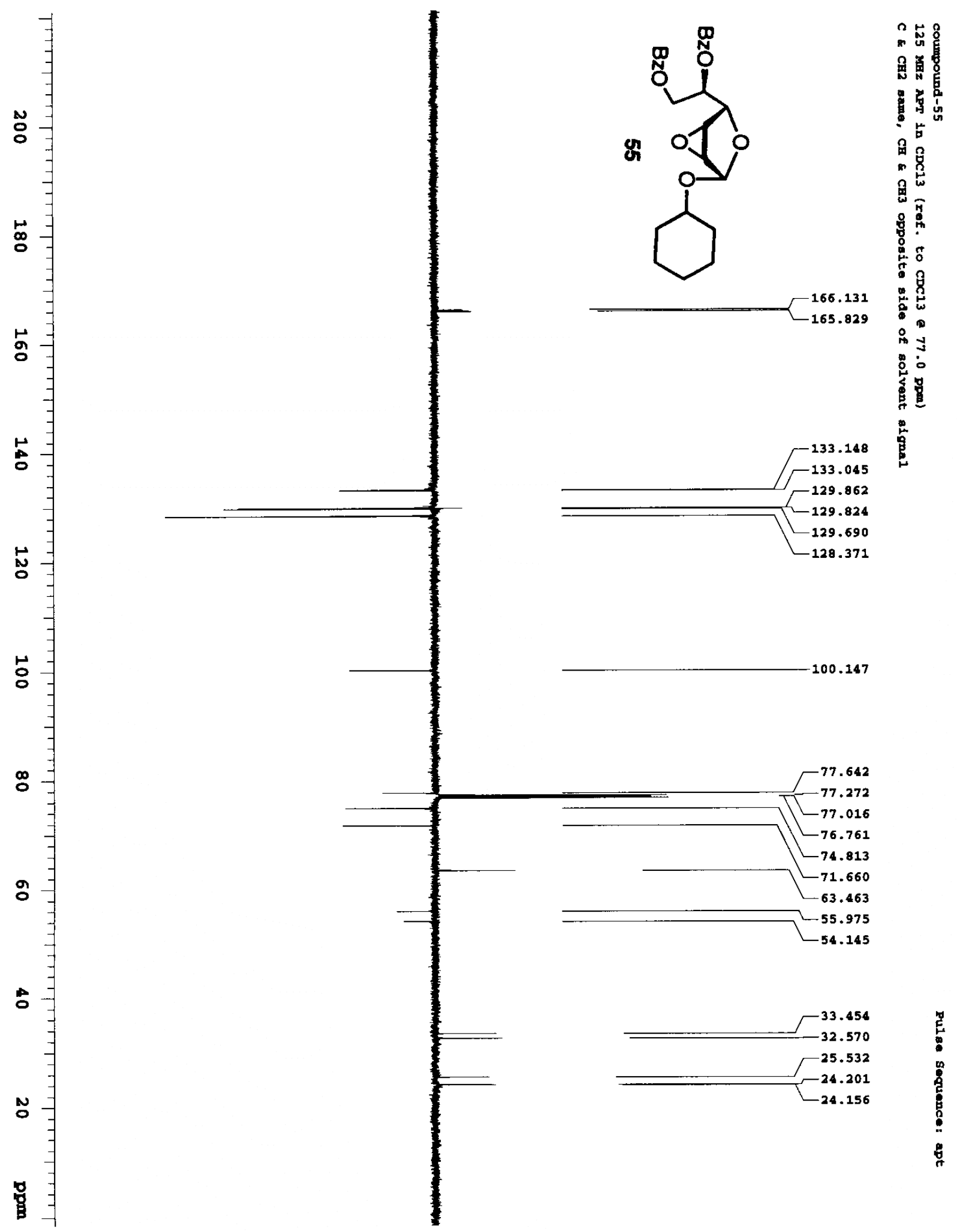




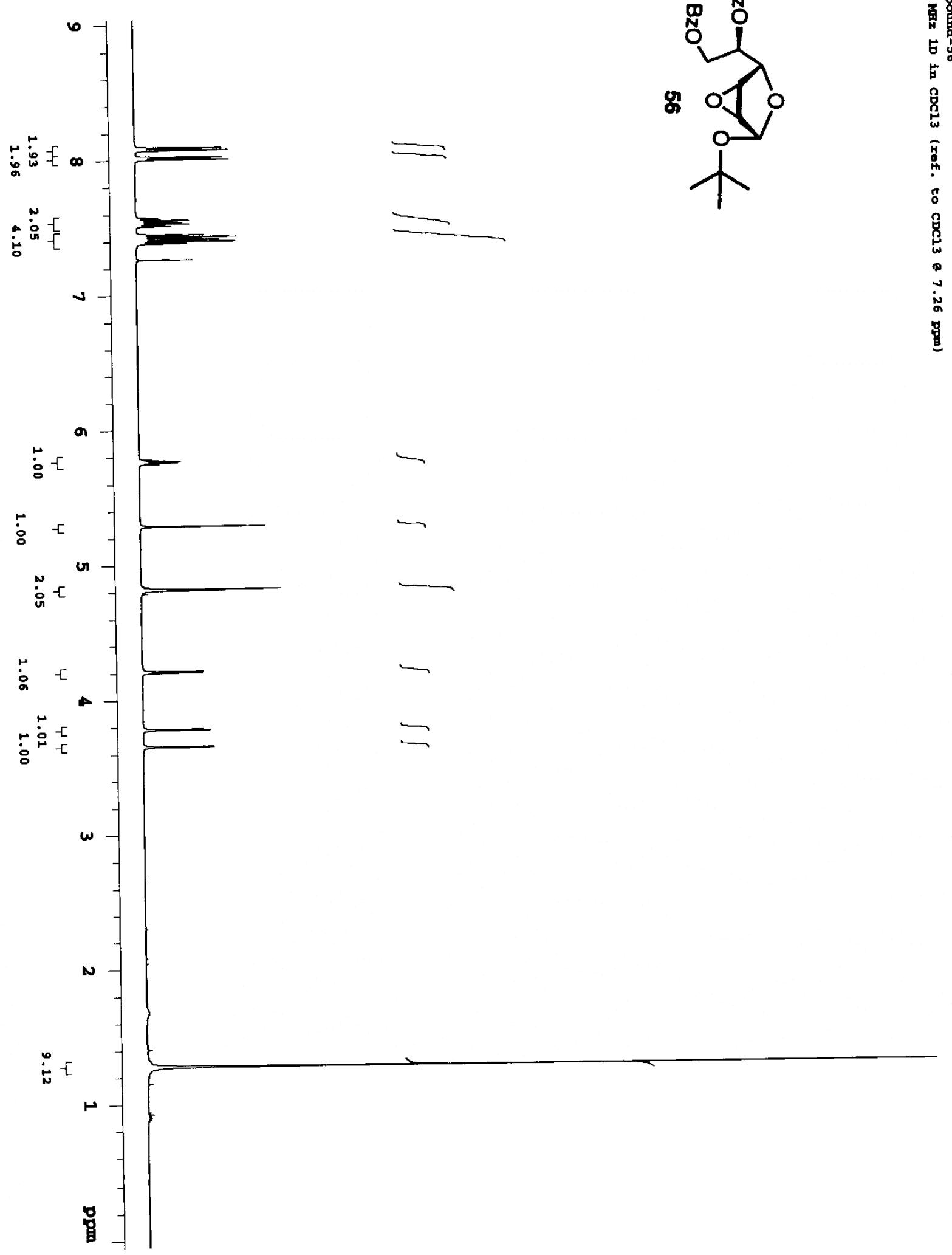




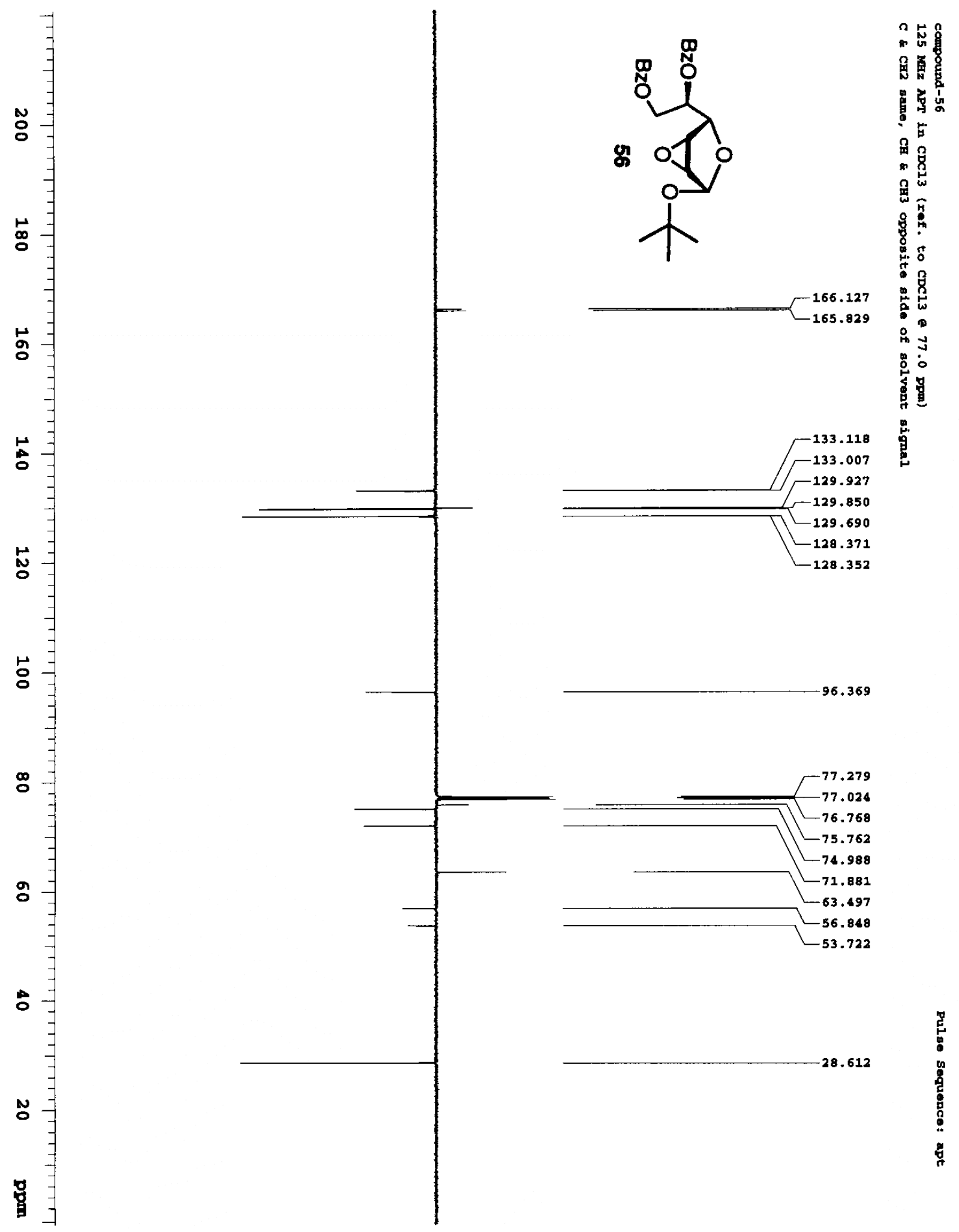



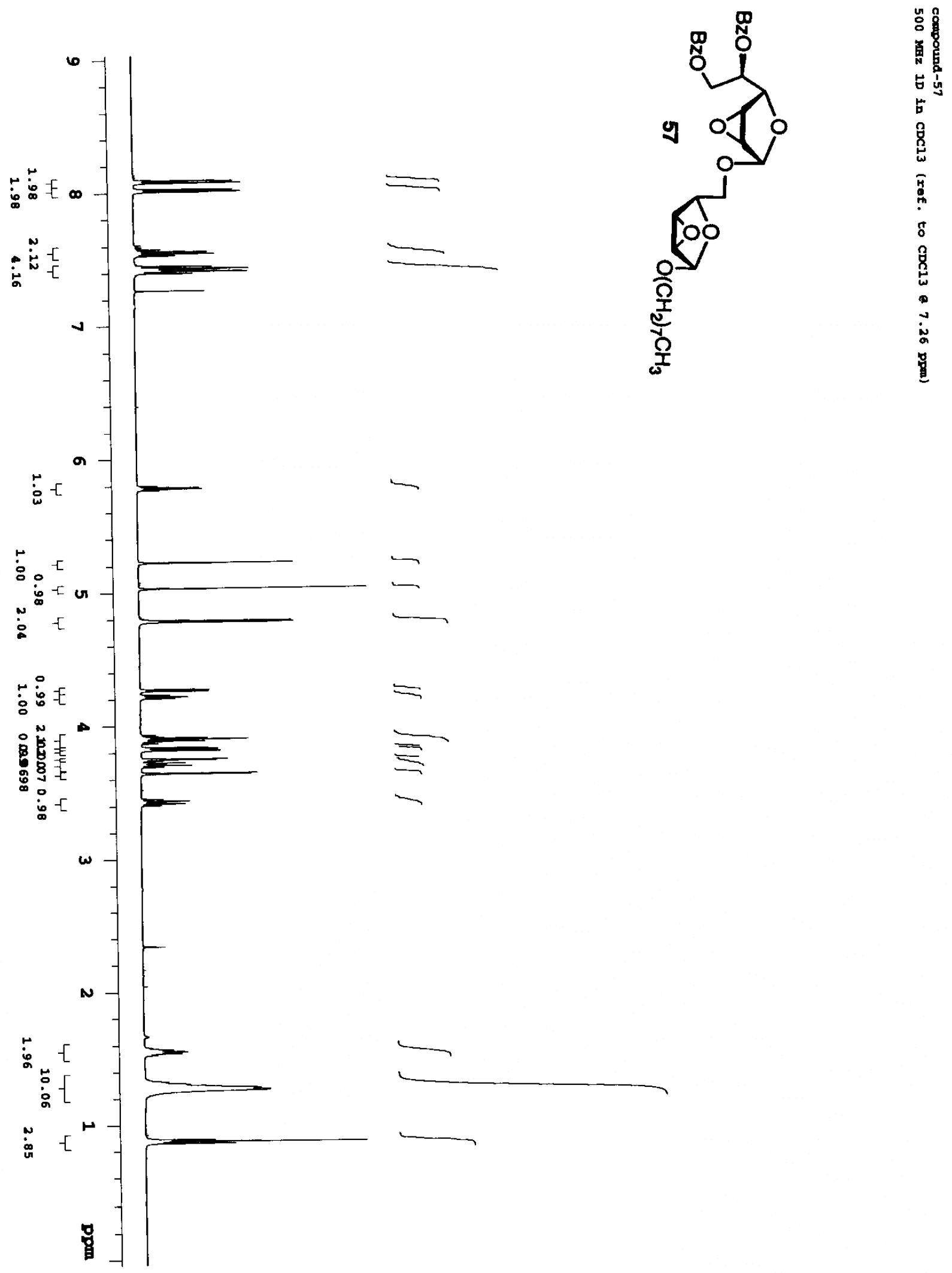


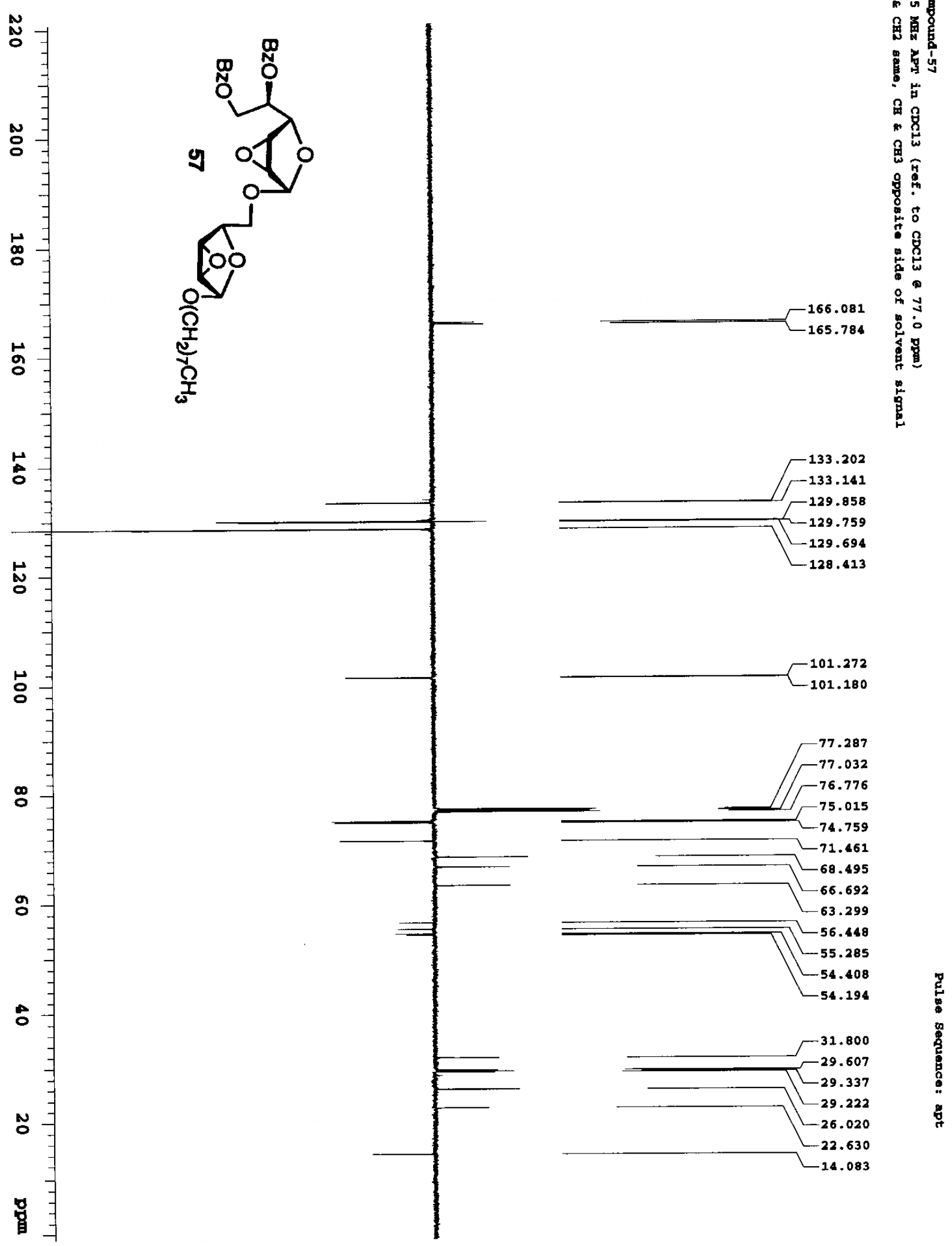




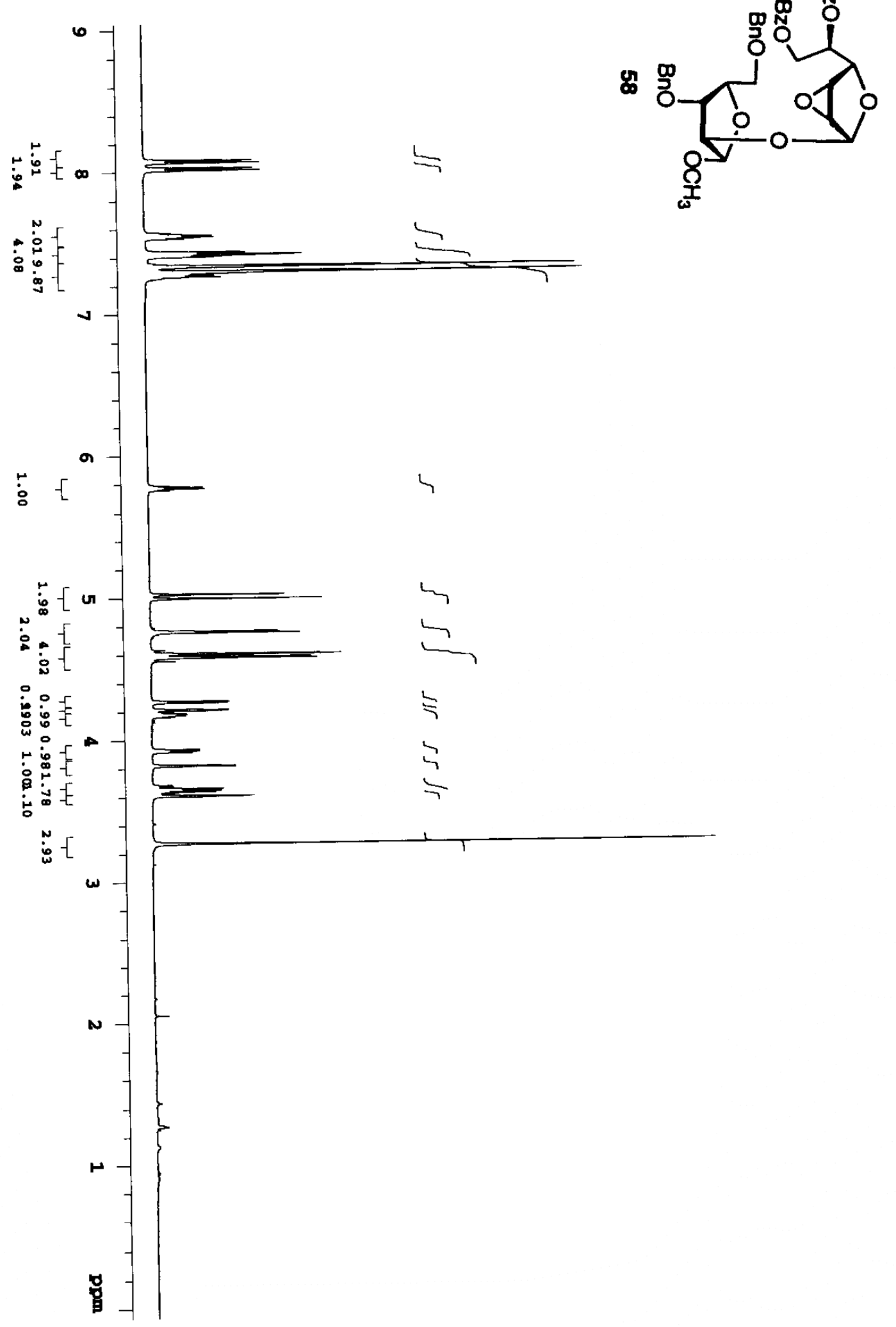

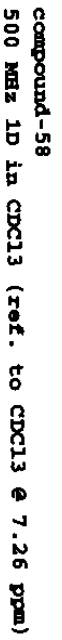




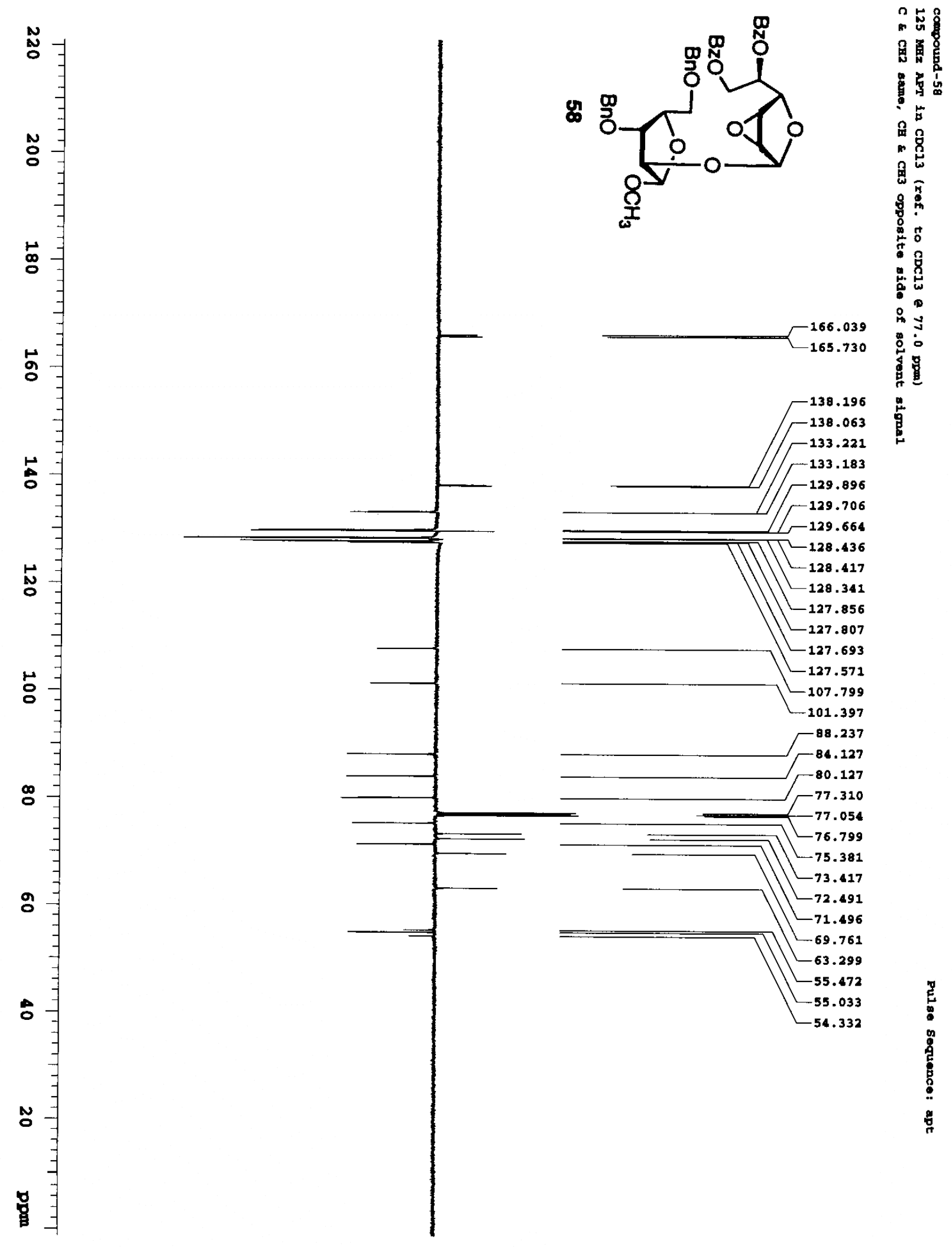




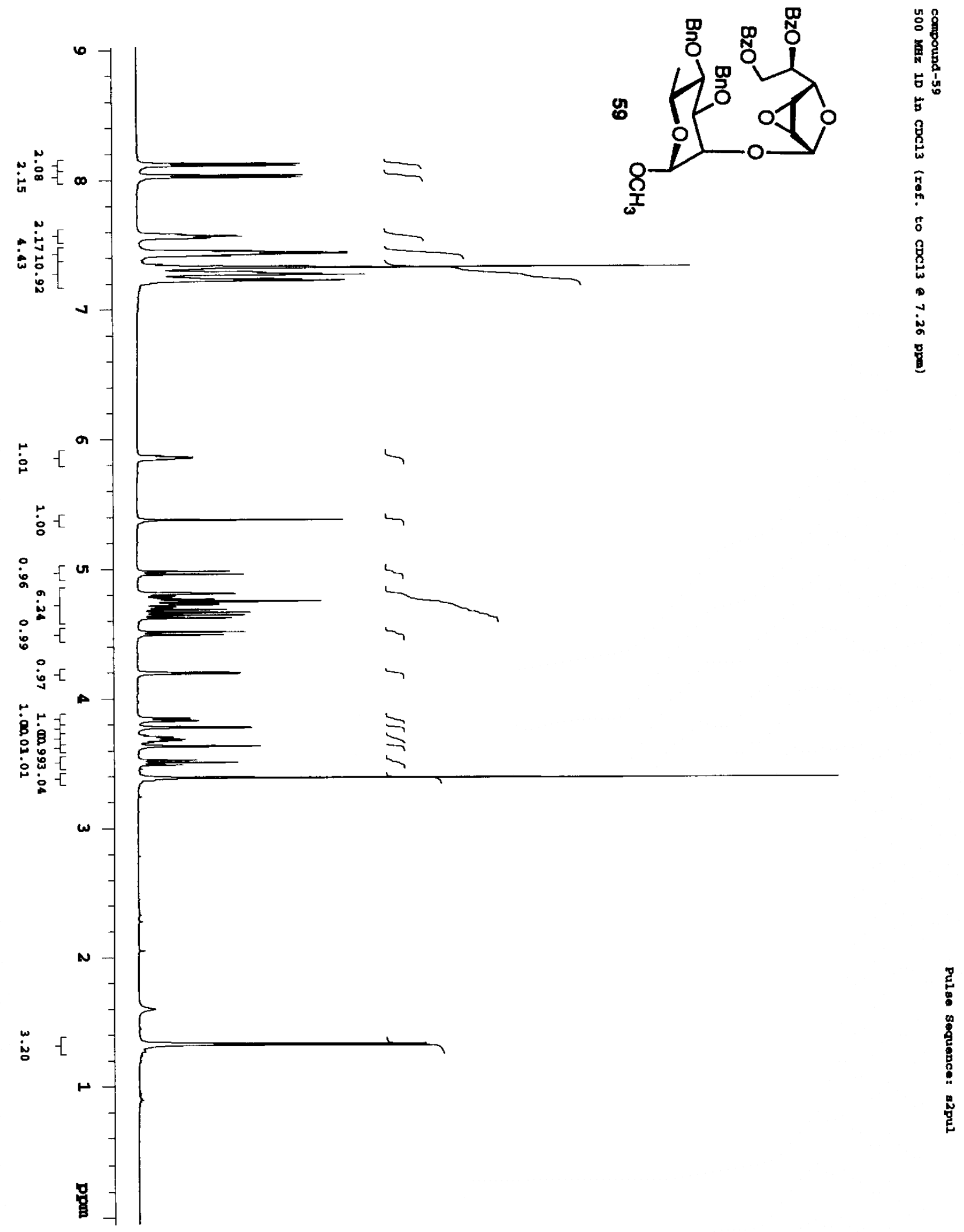



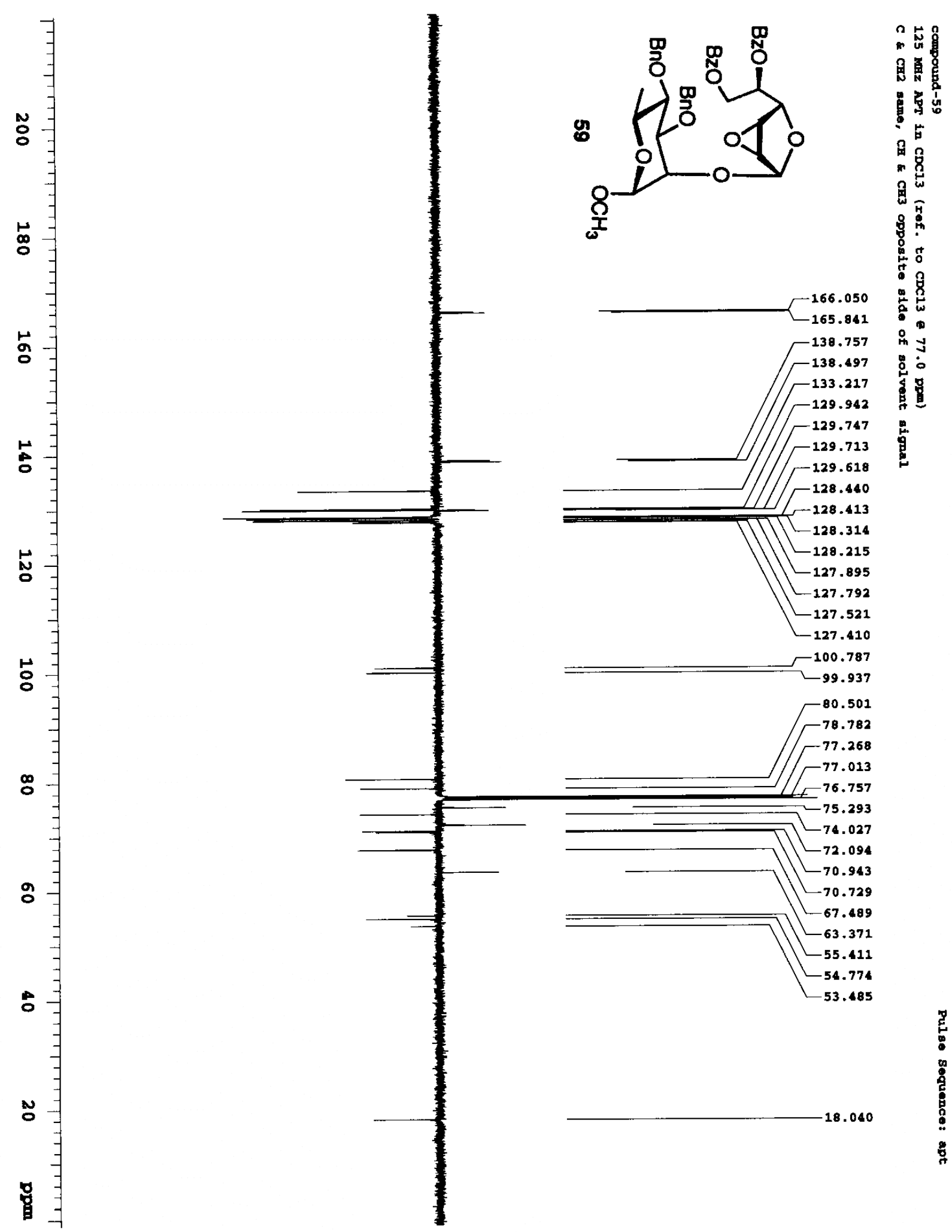


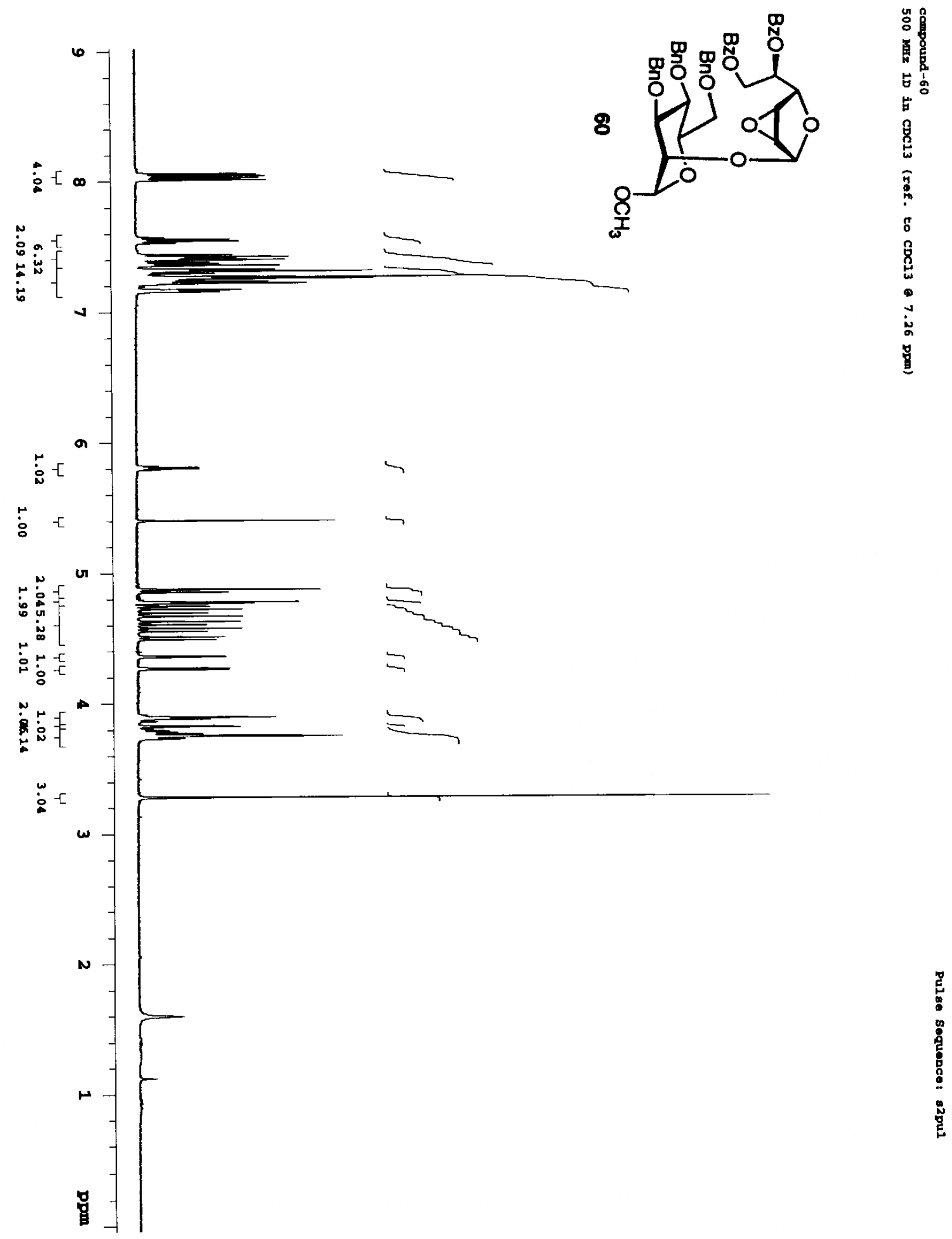




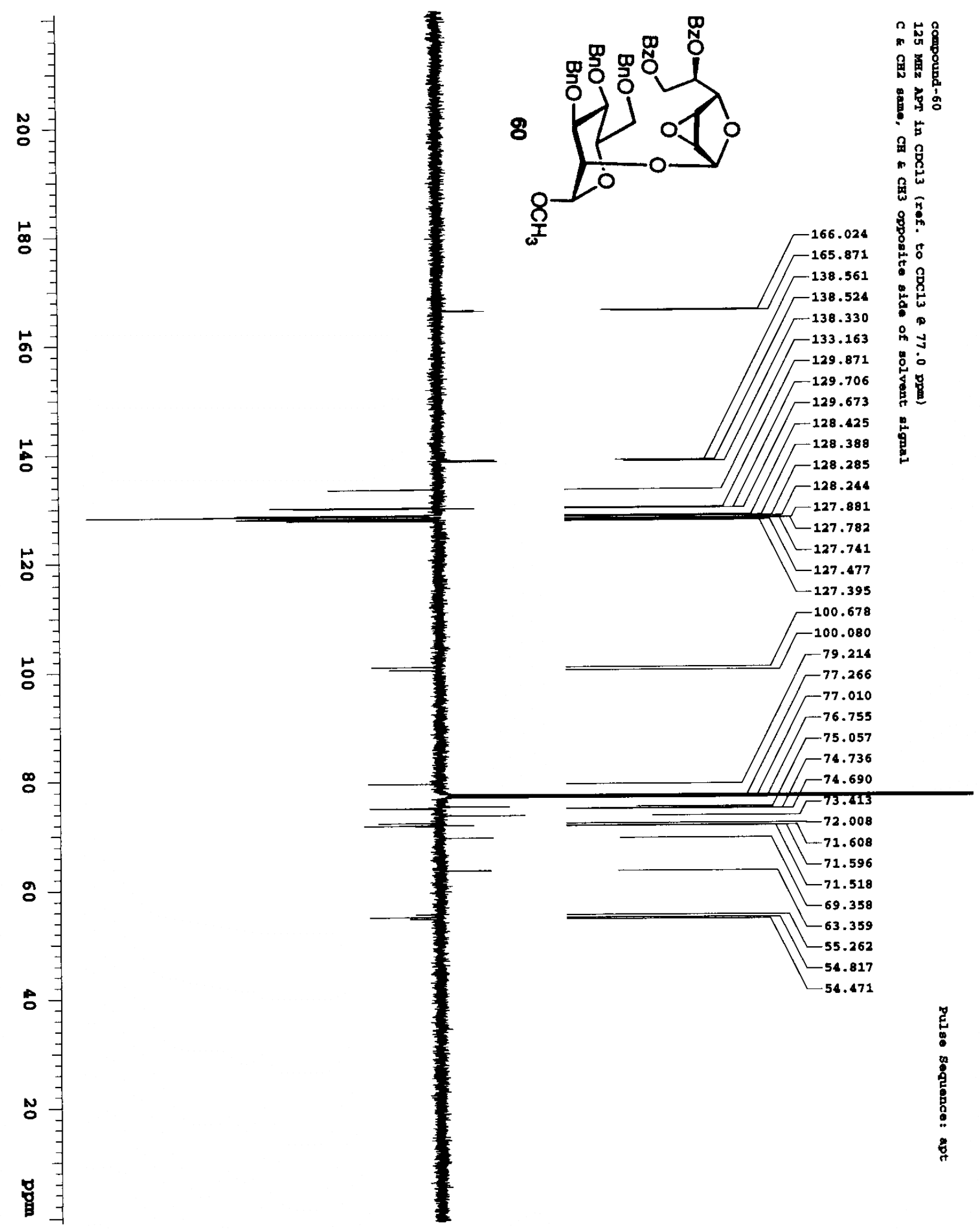




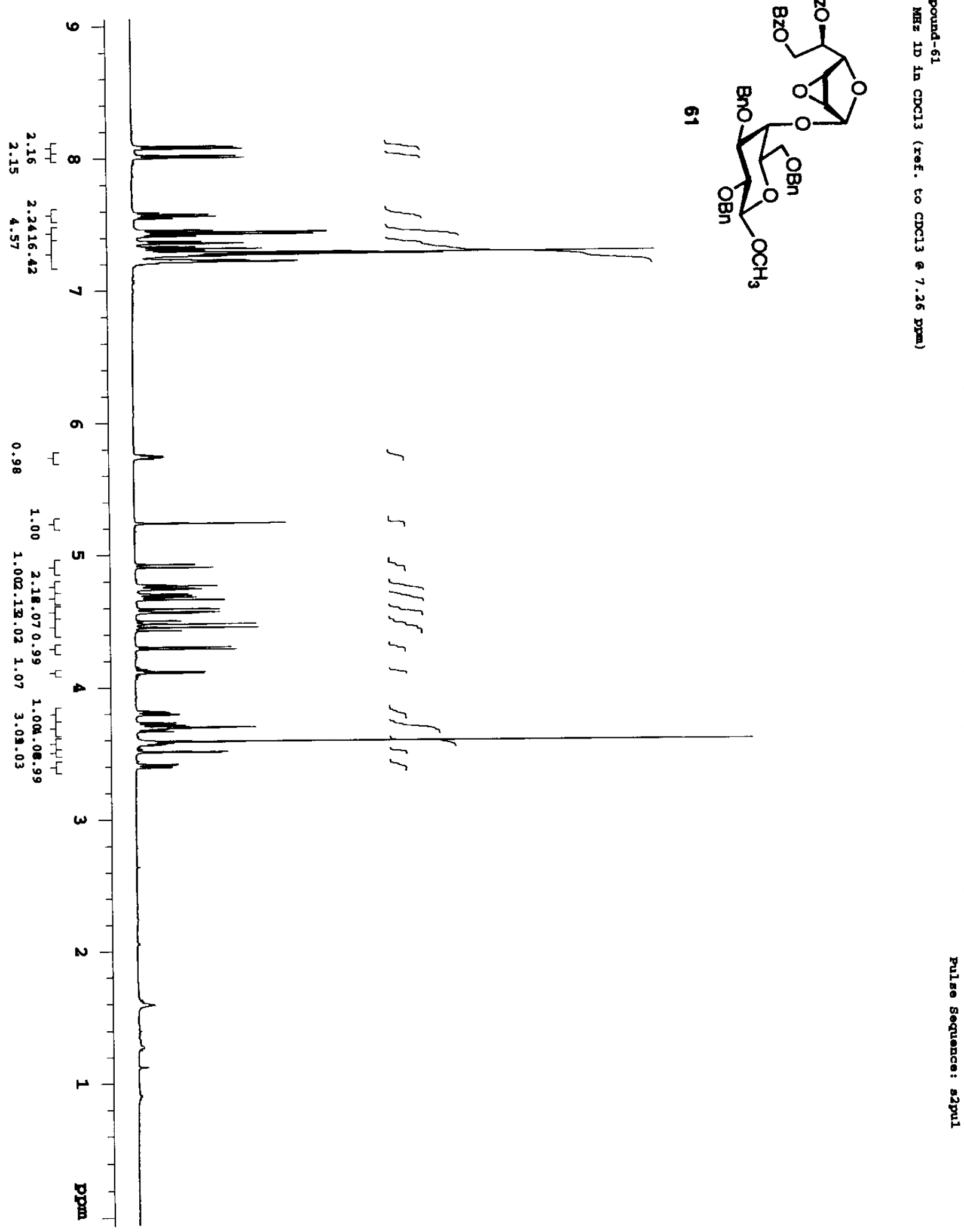




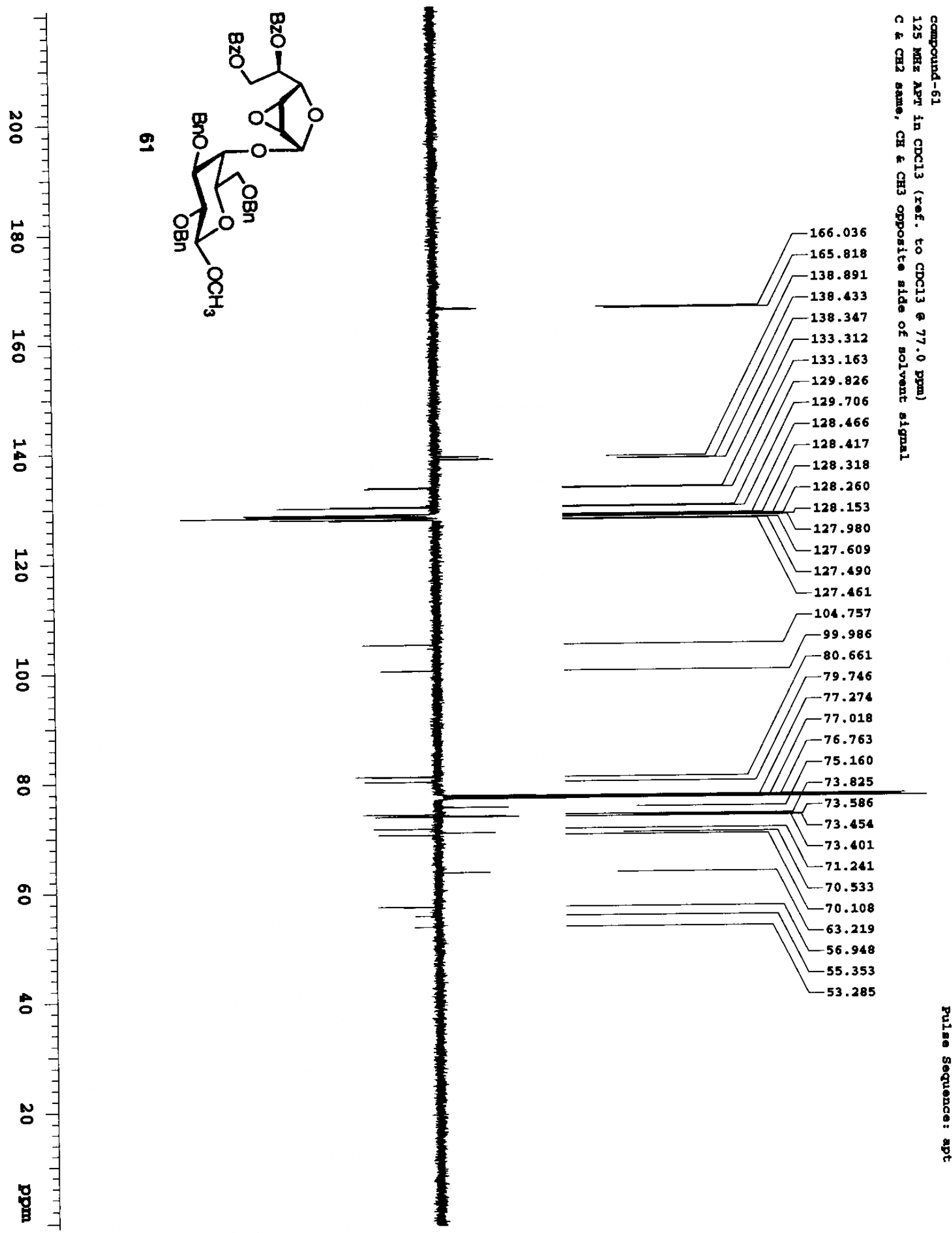



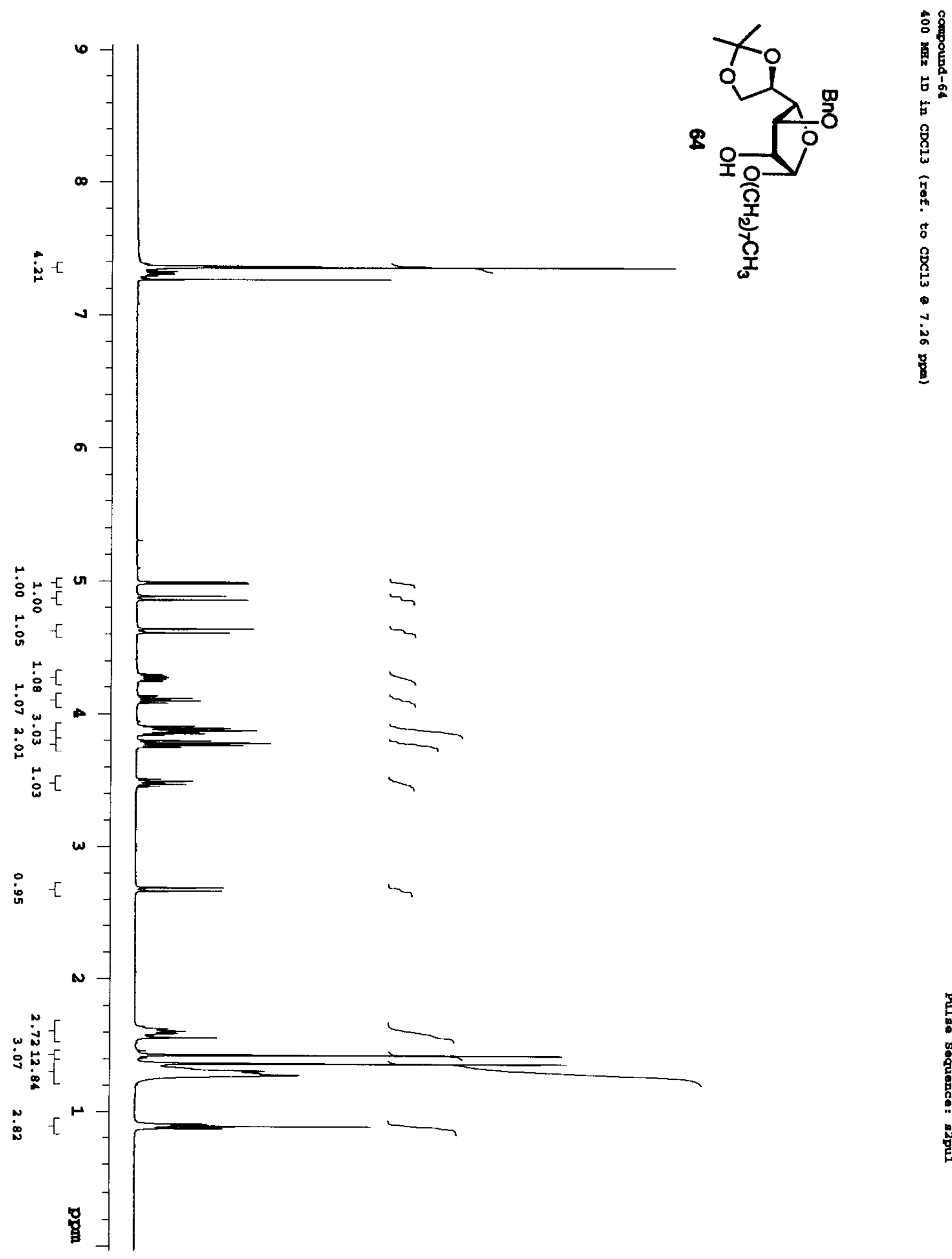


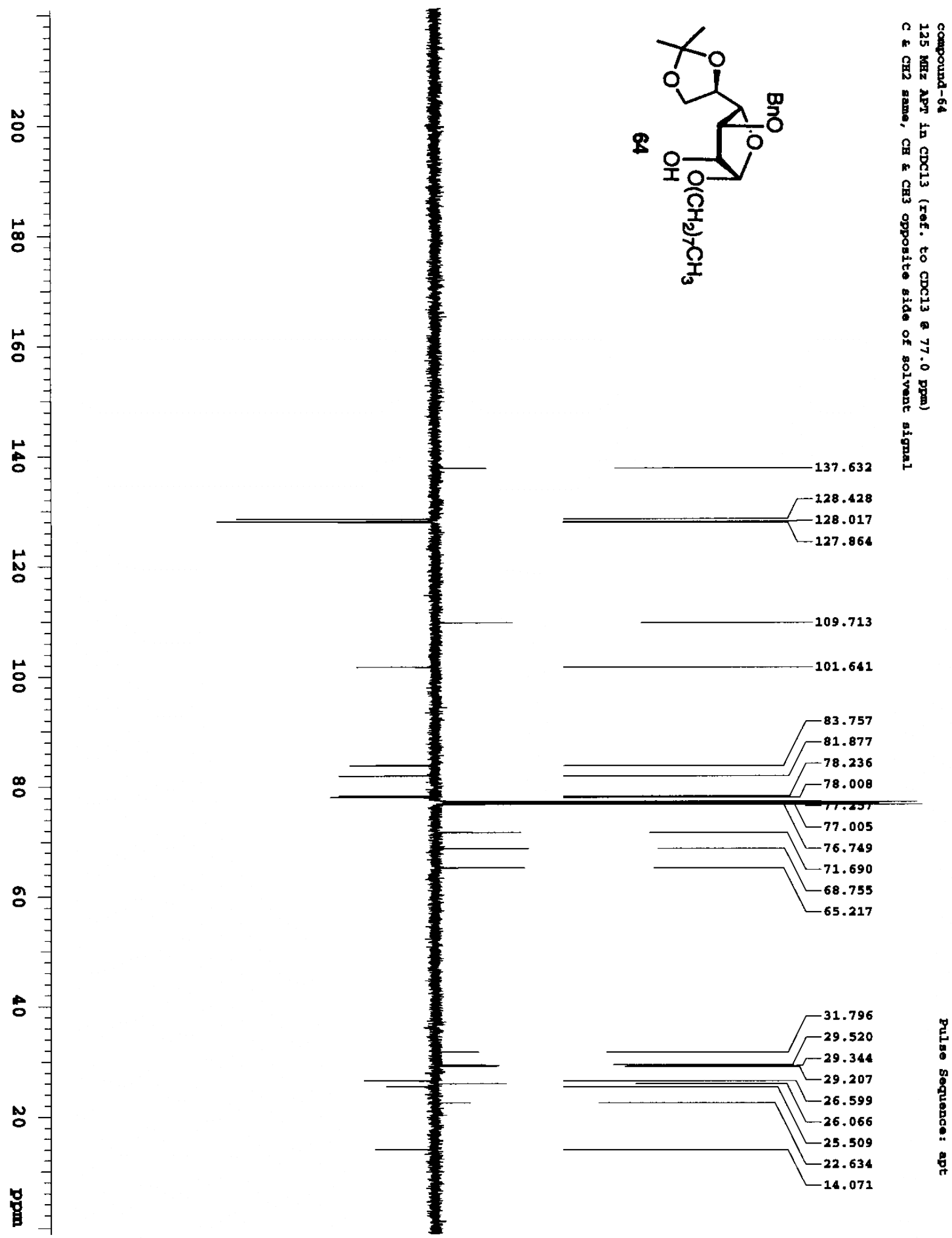




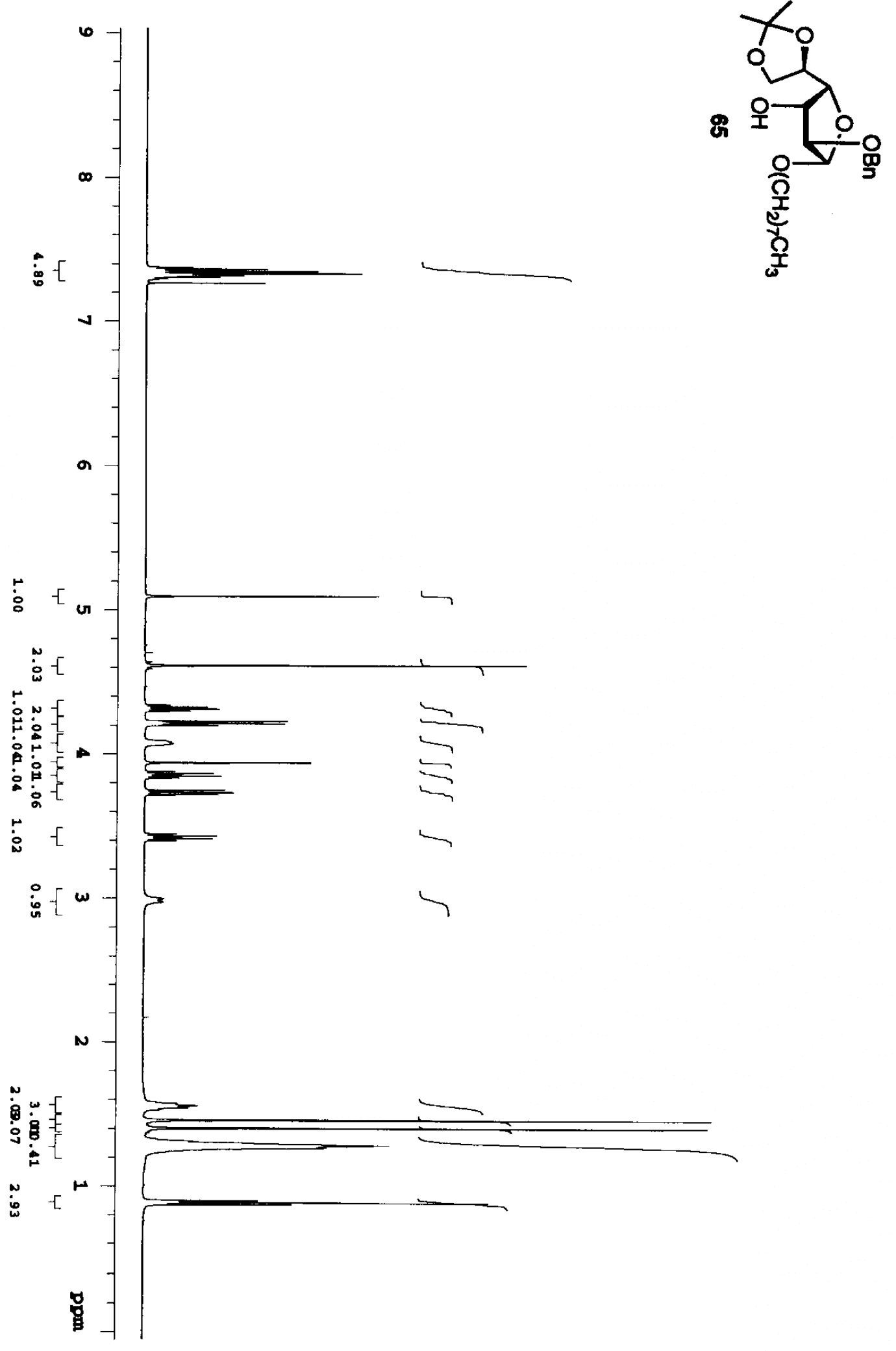

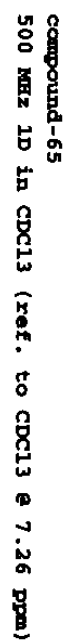




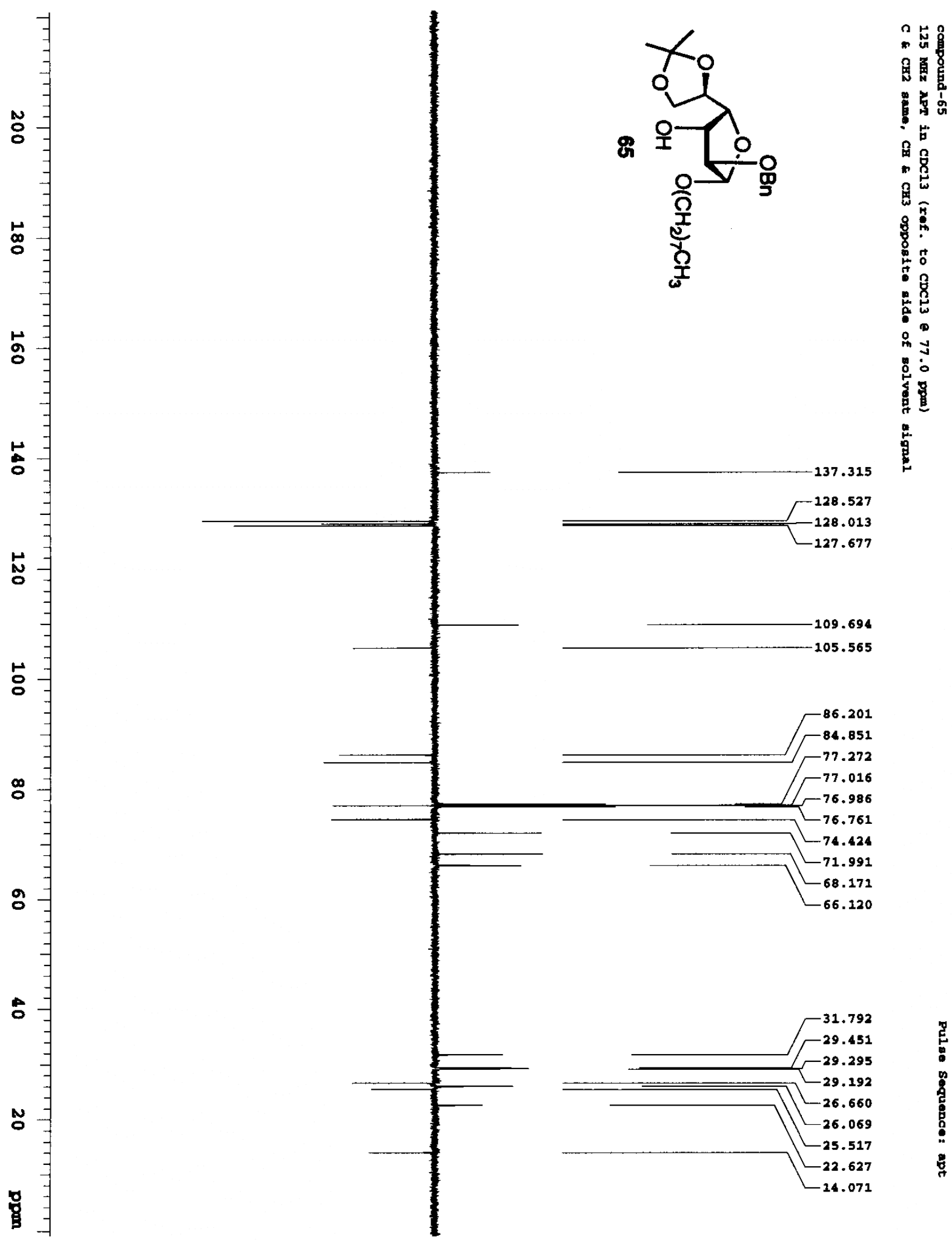



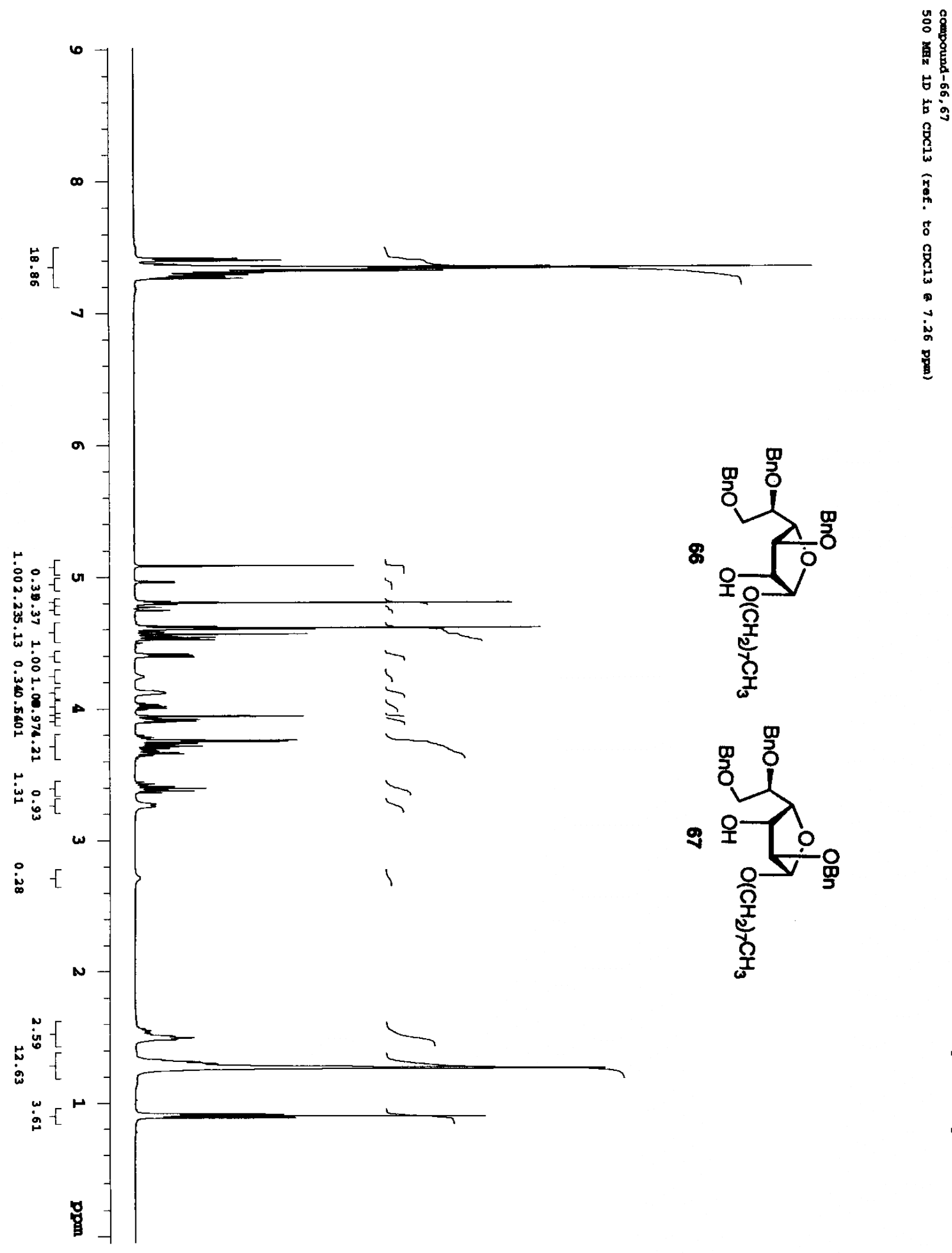


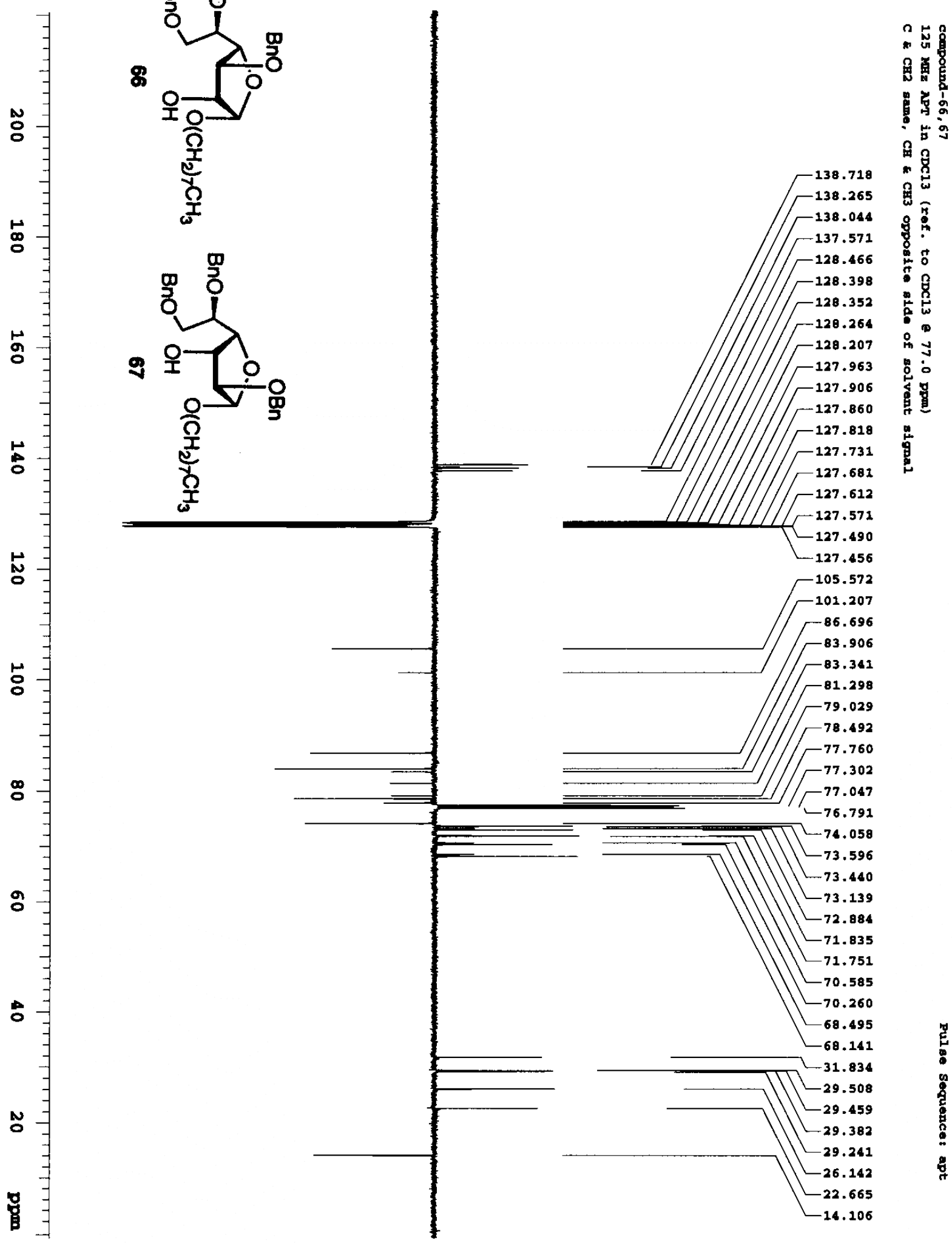




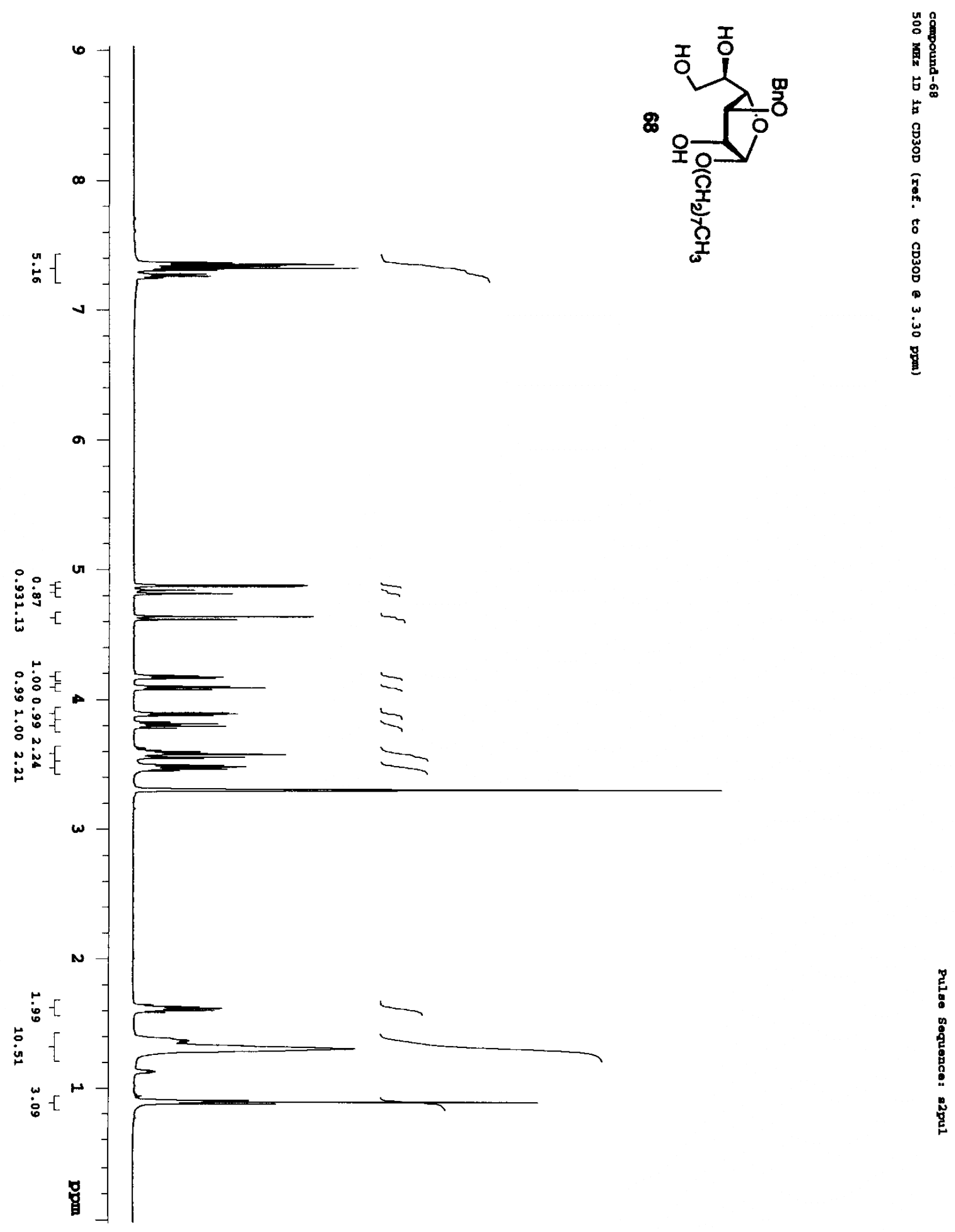




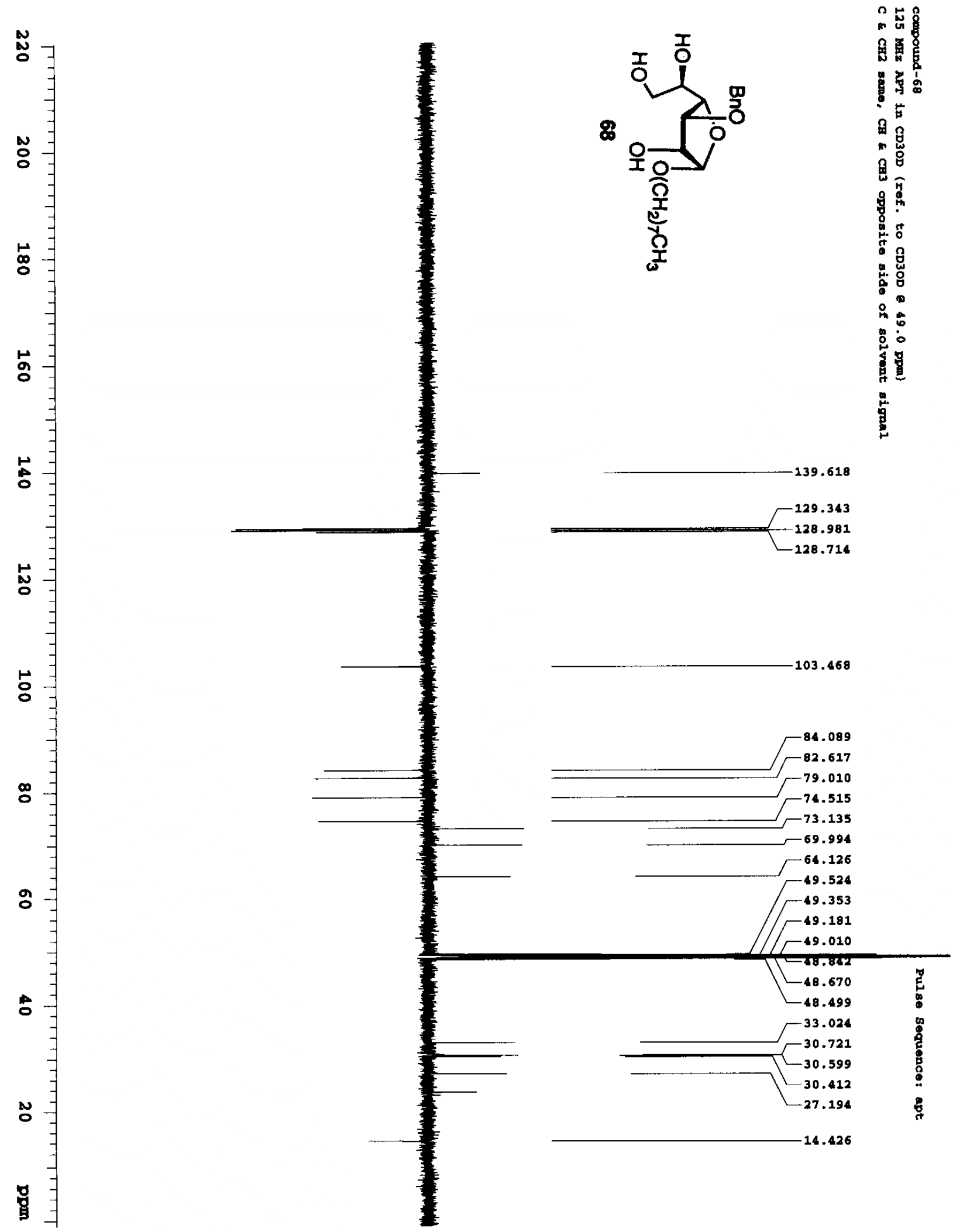



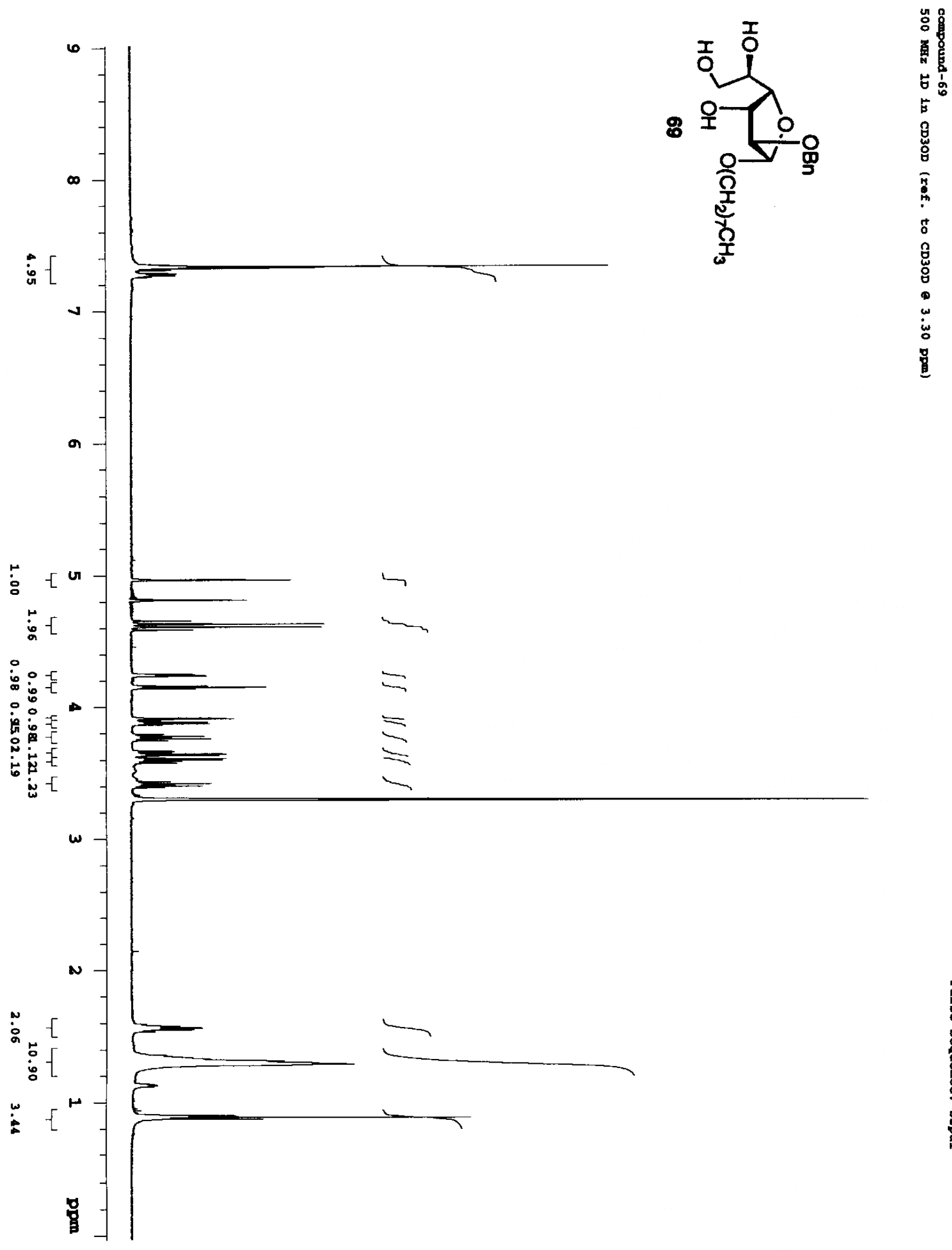


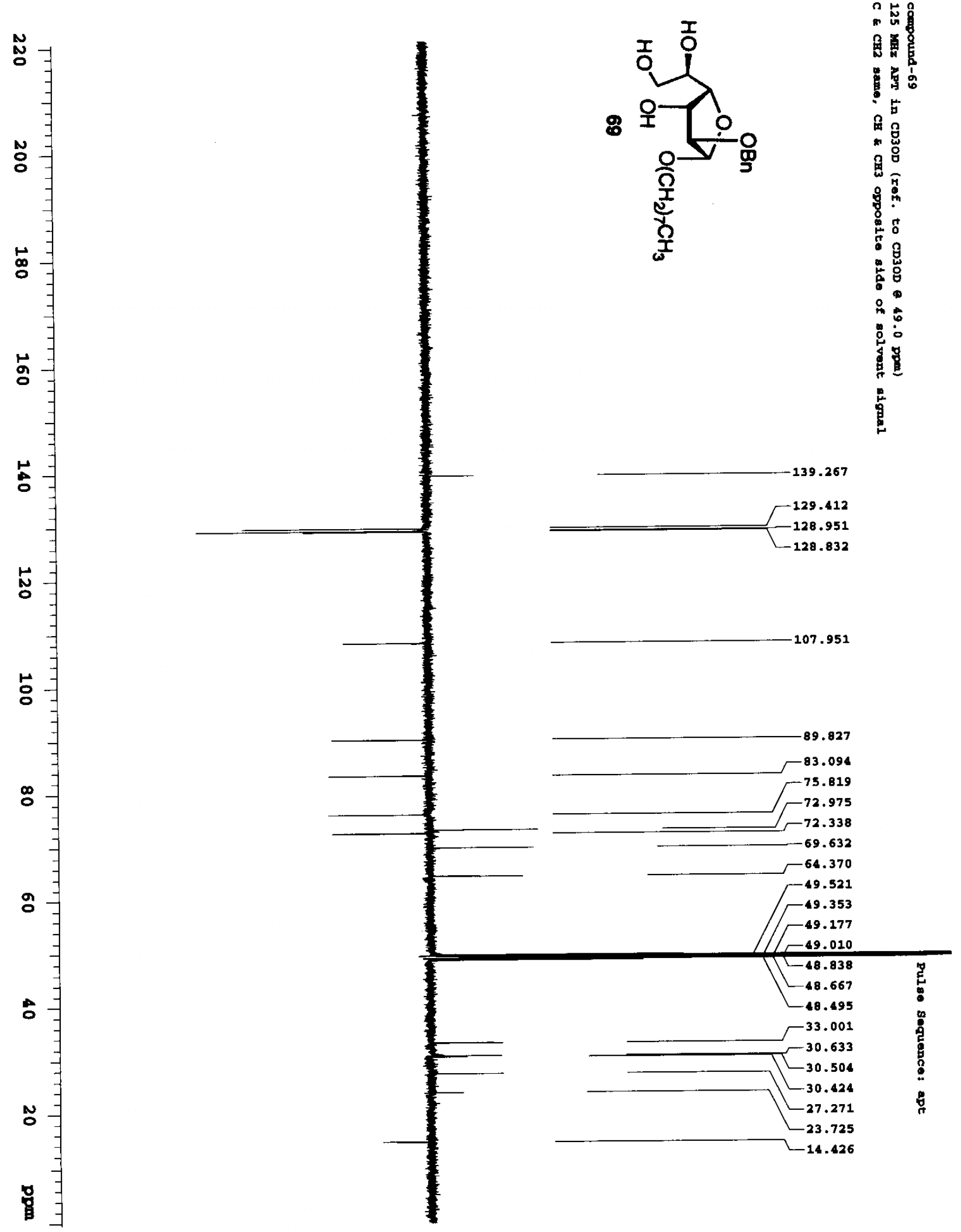



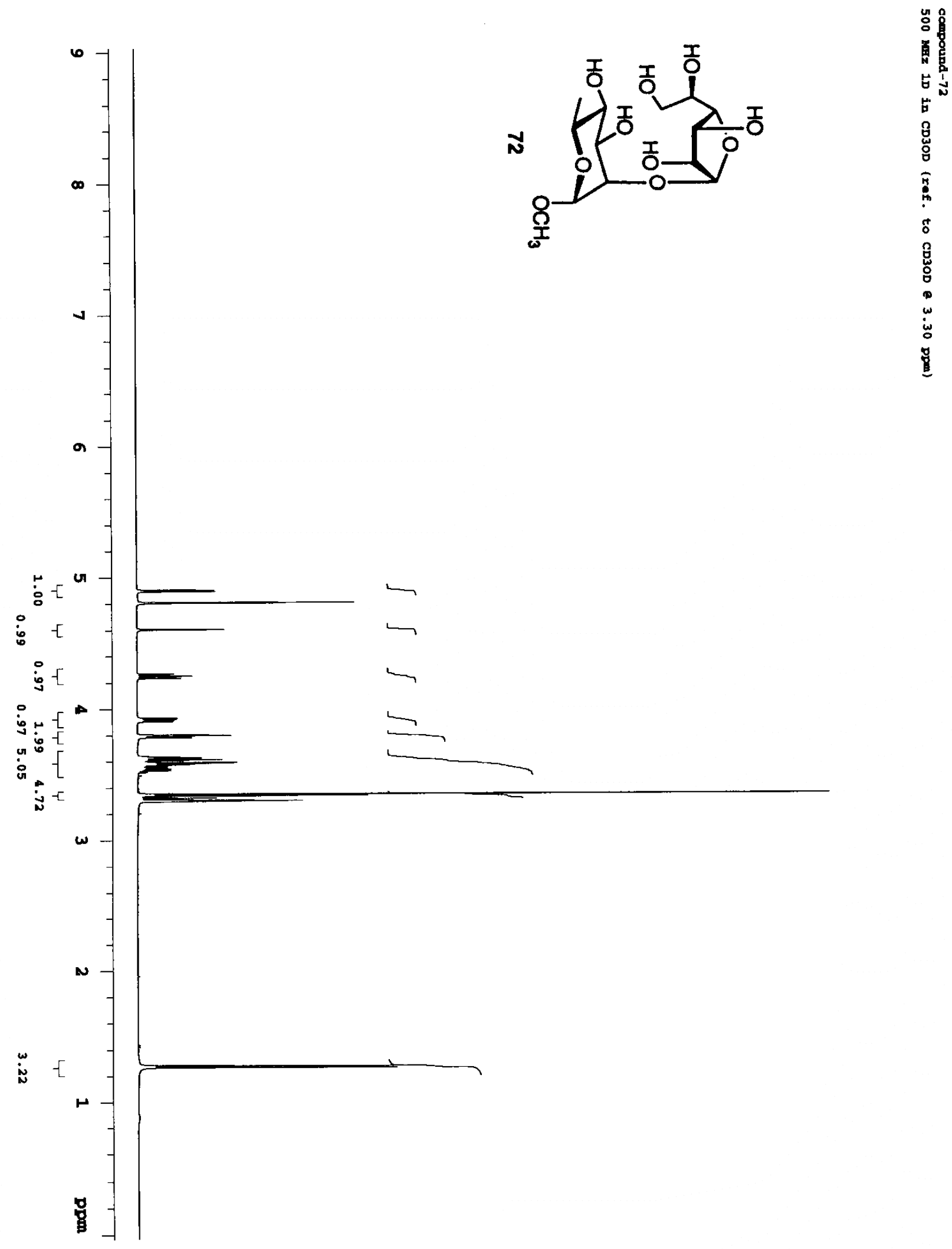


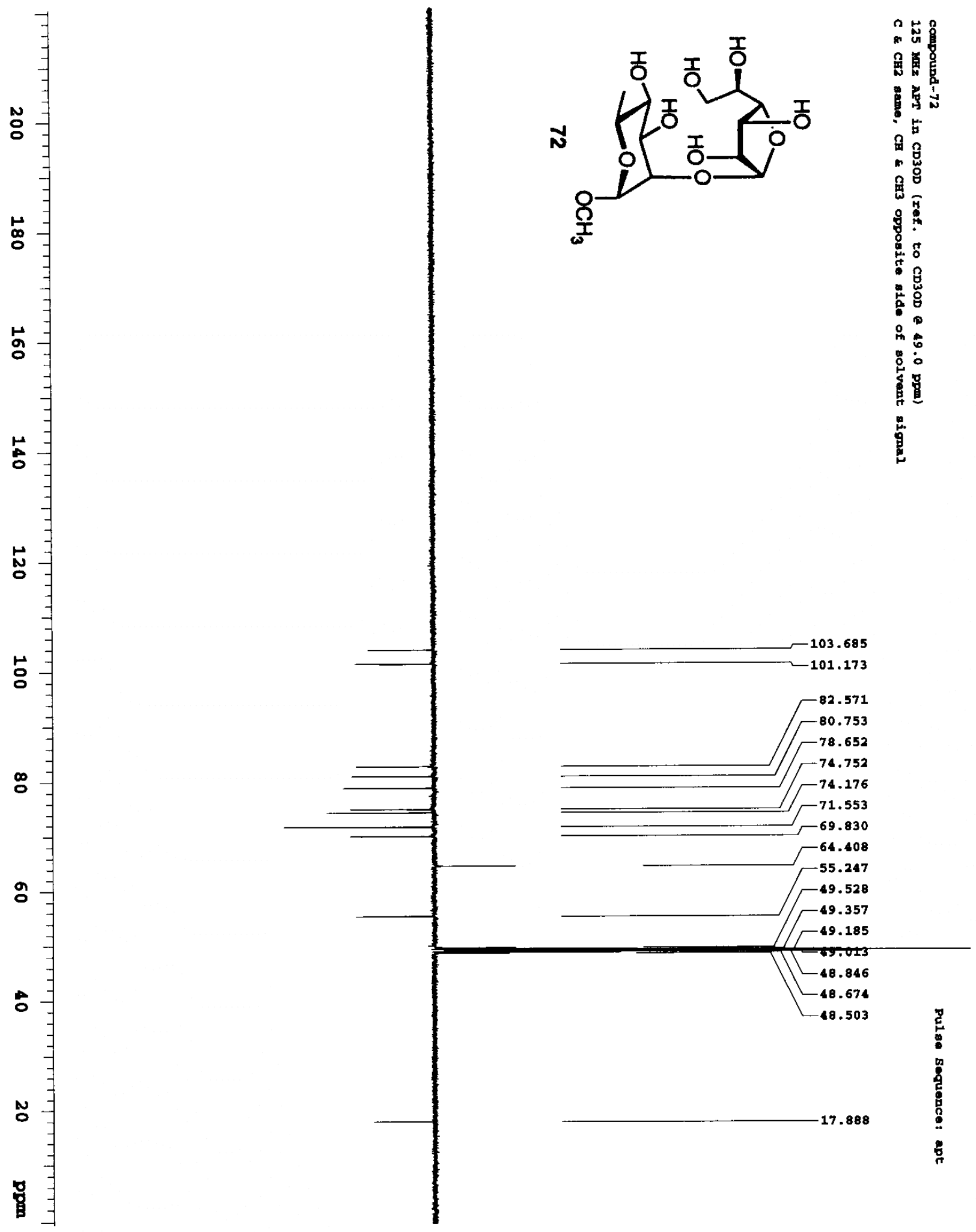




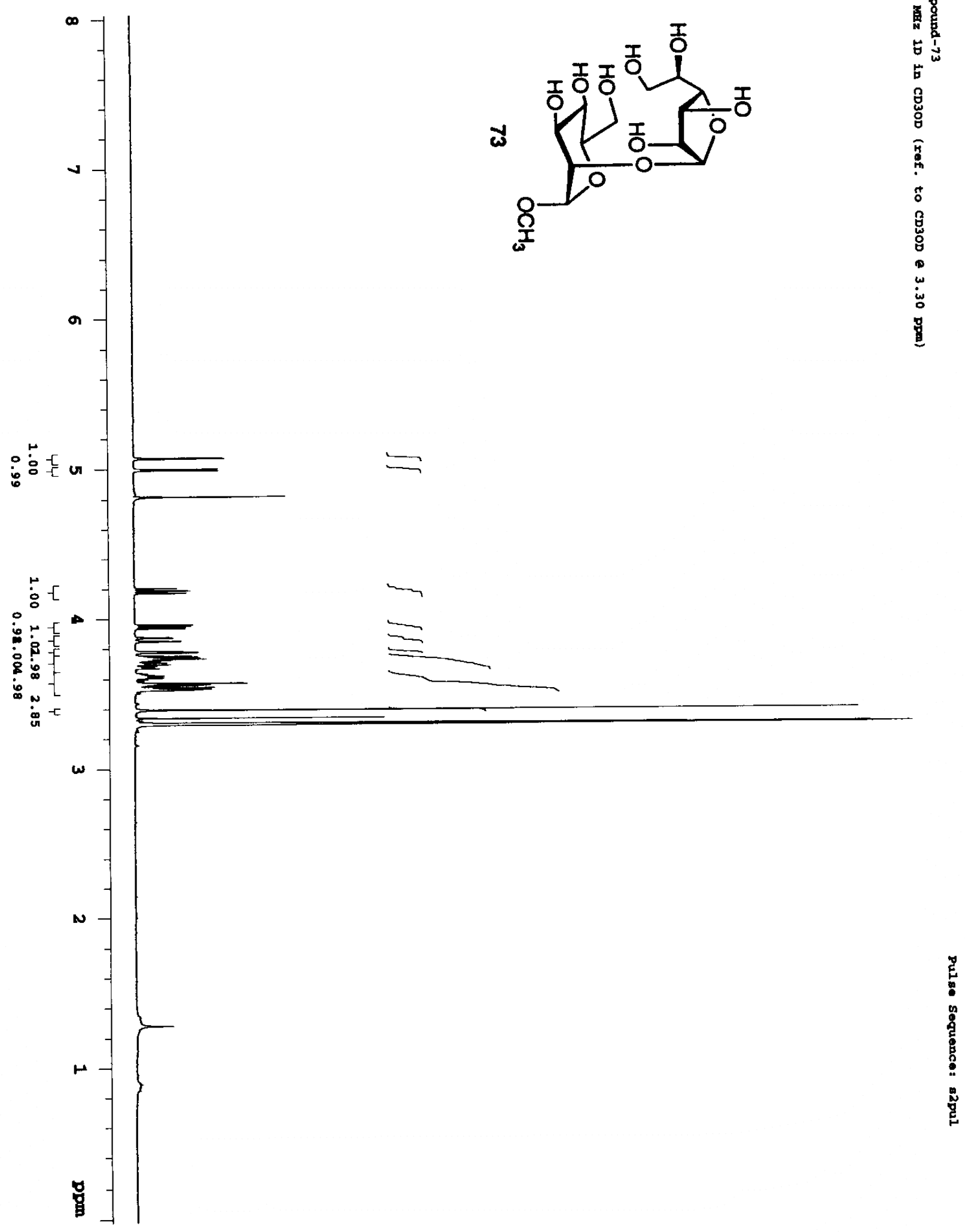



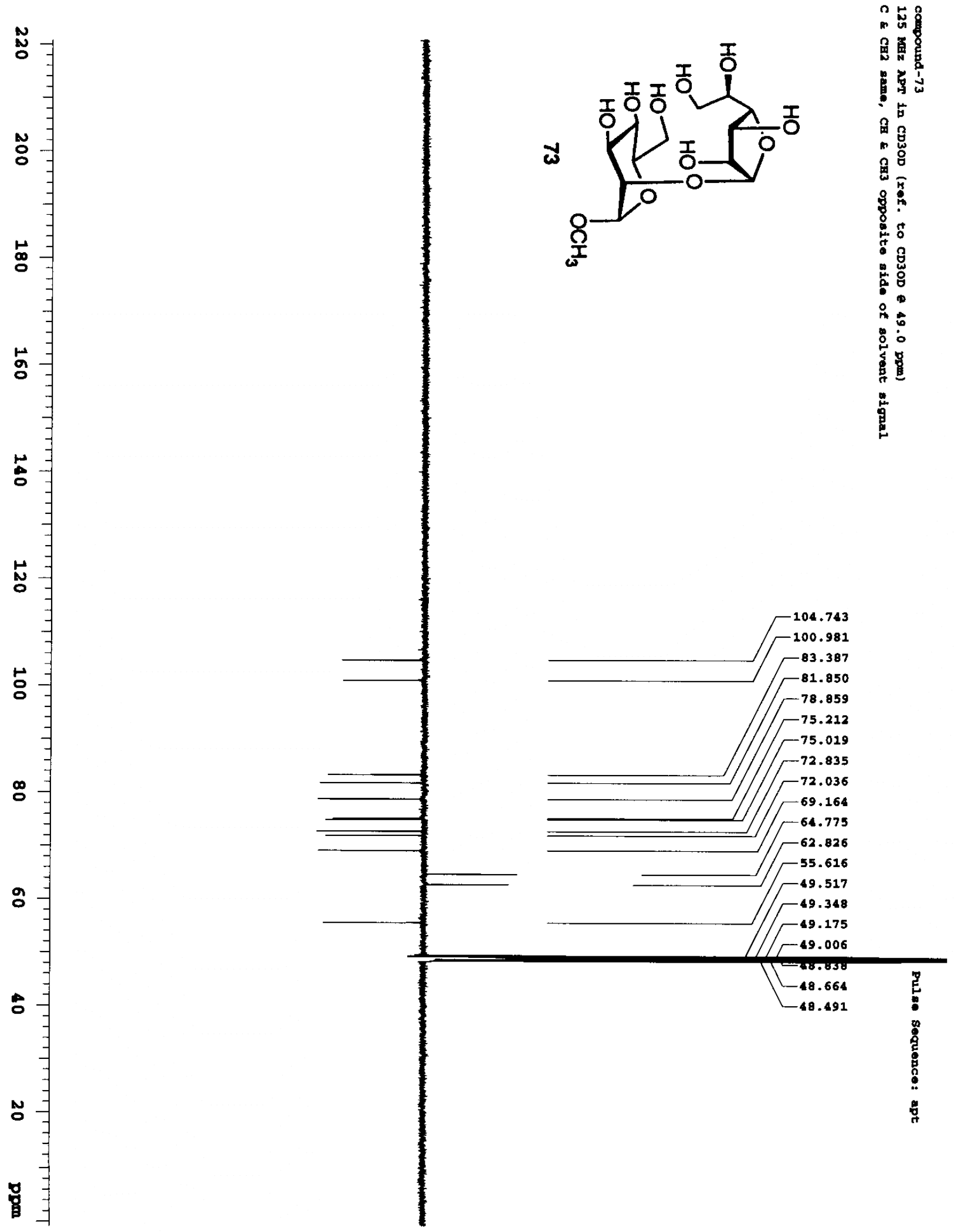


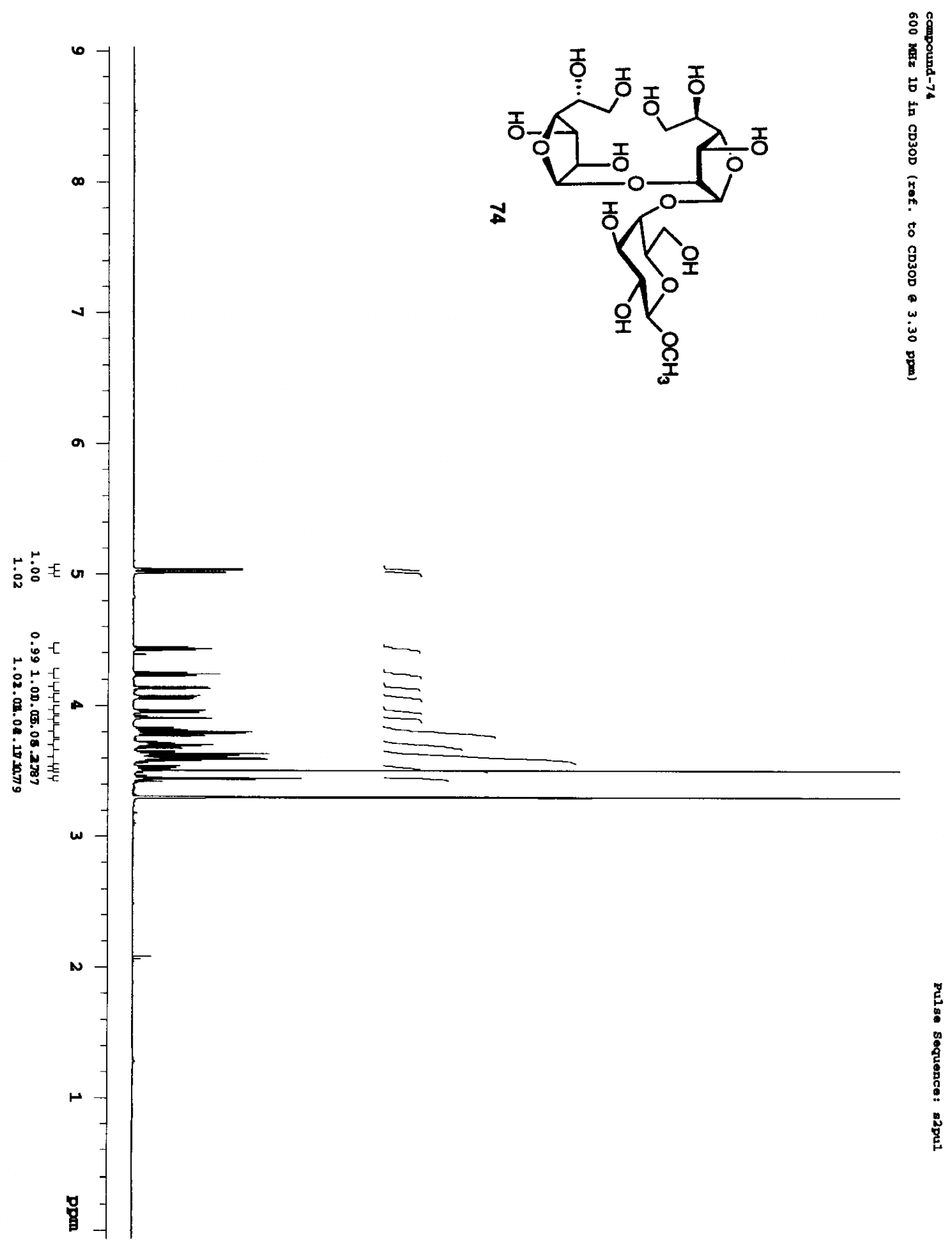




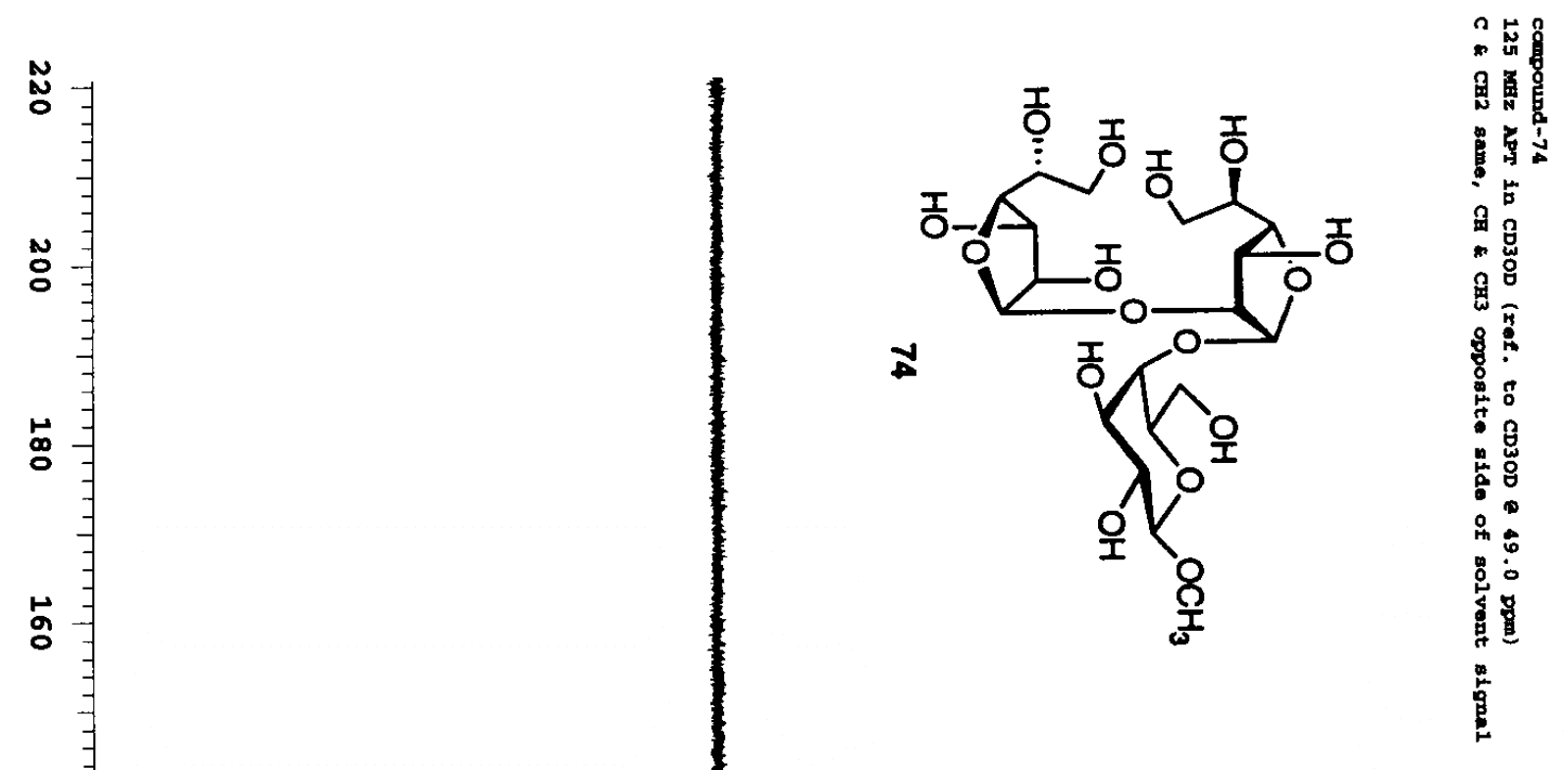

농

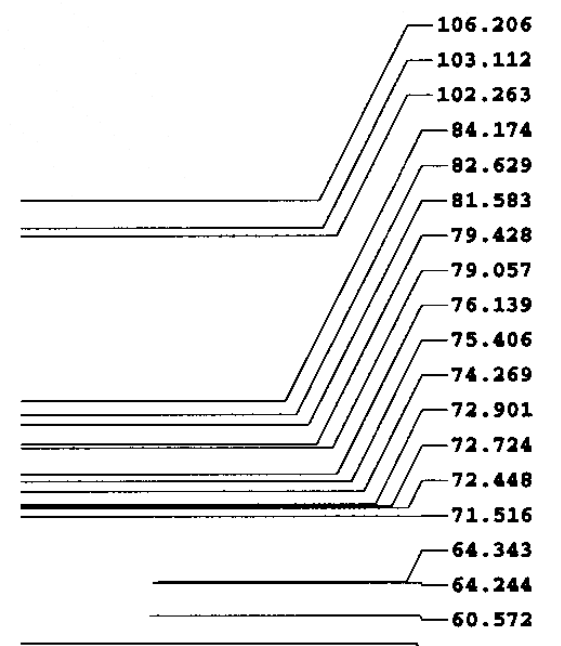

o

$\stackrel{\infty}{\circ}$

용

点

过

.
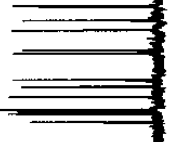

:

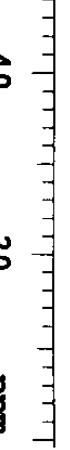

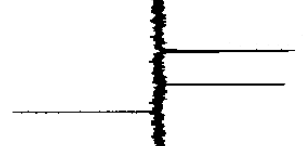

$-57.544$ $-49.522$

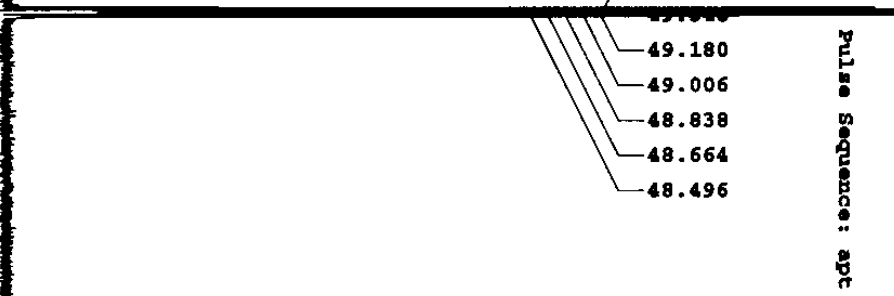




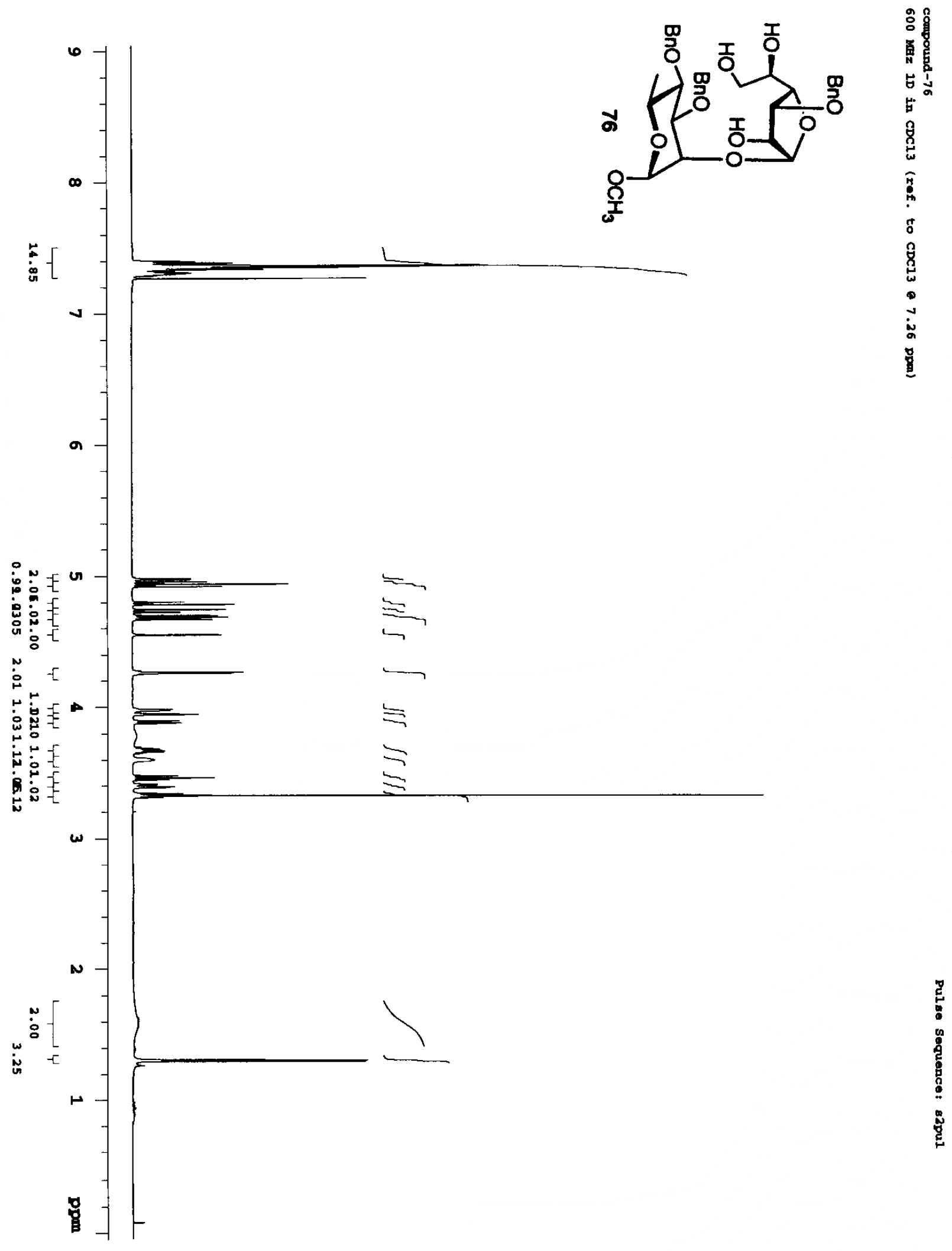



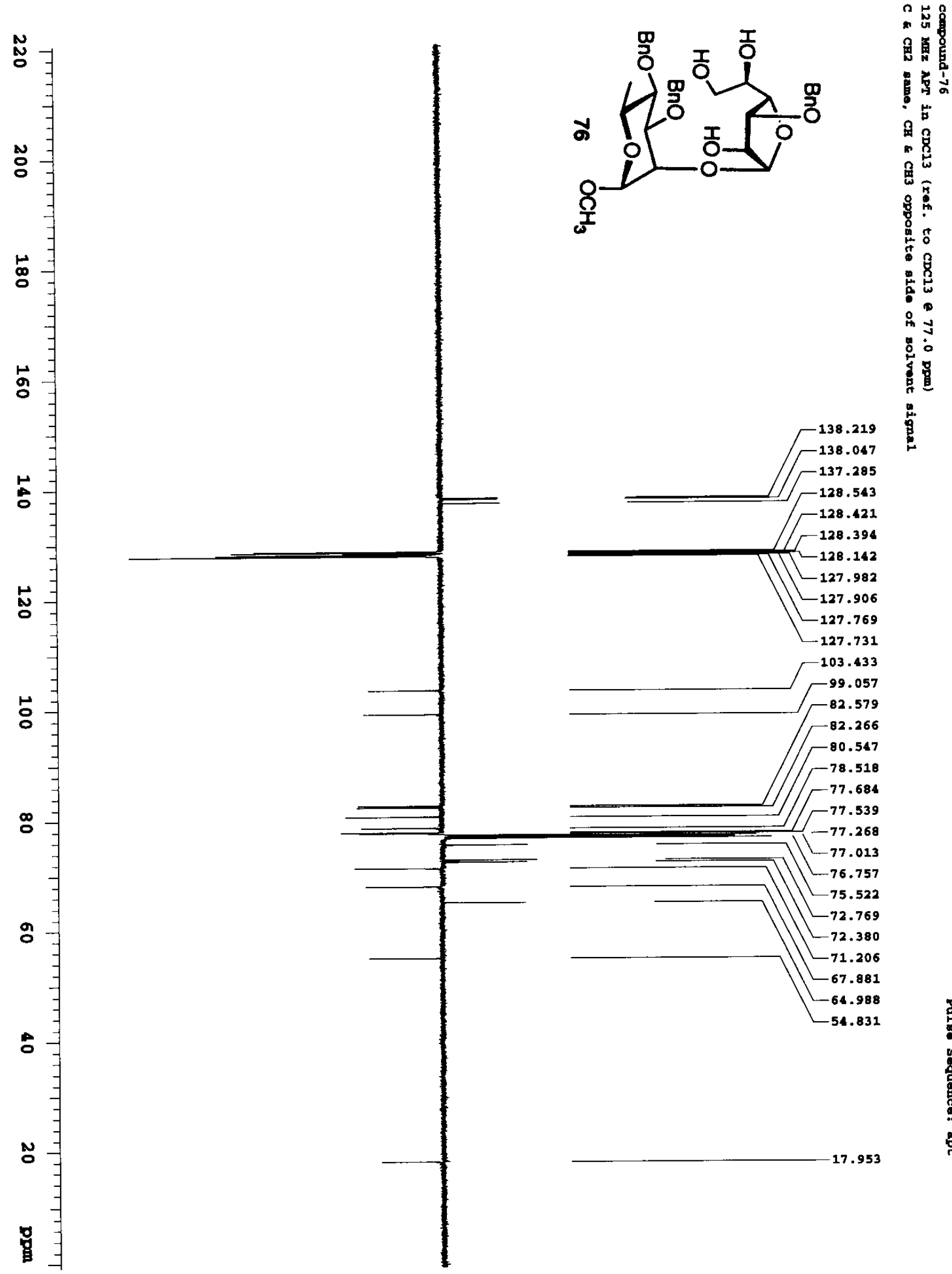


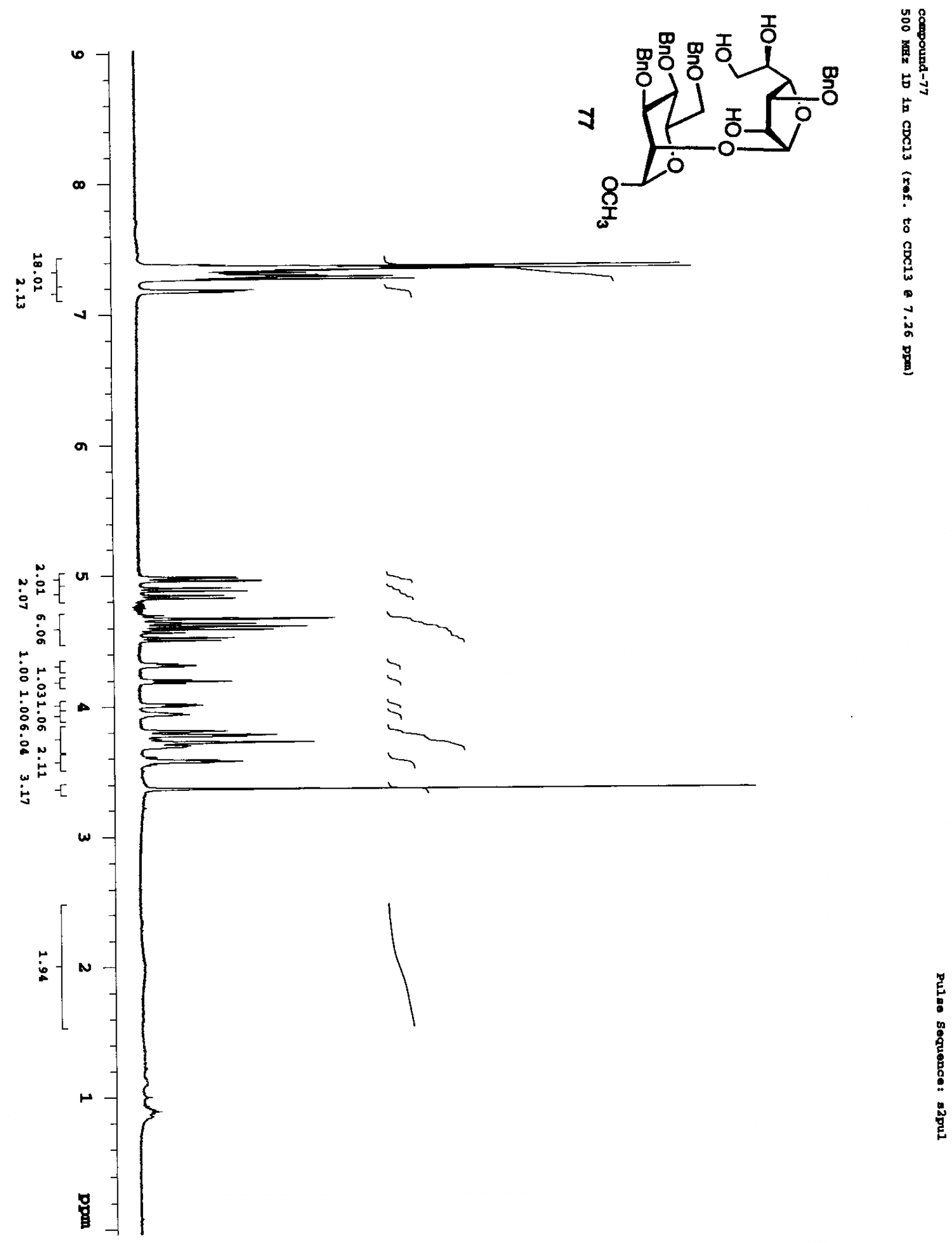




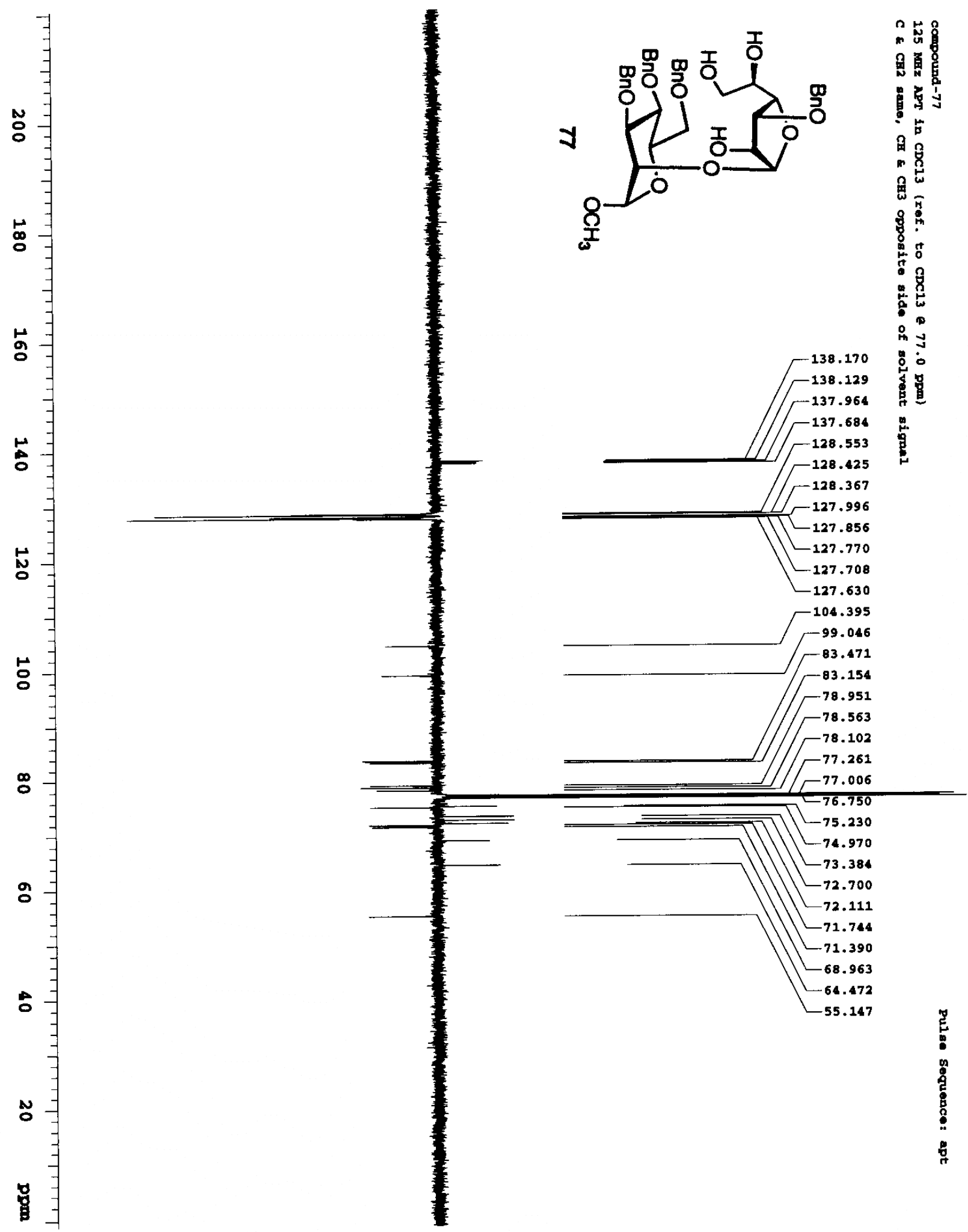




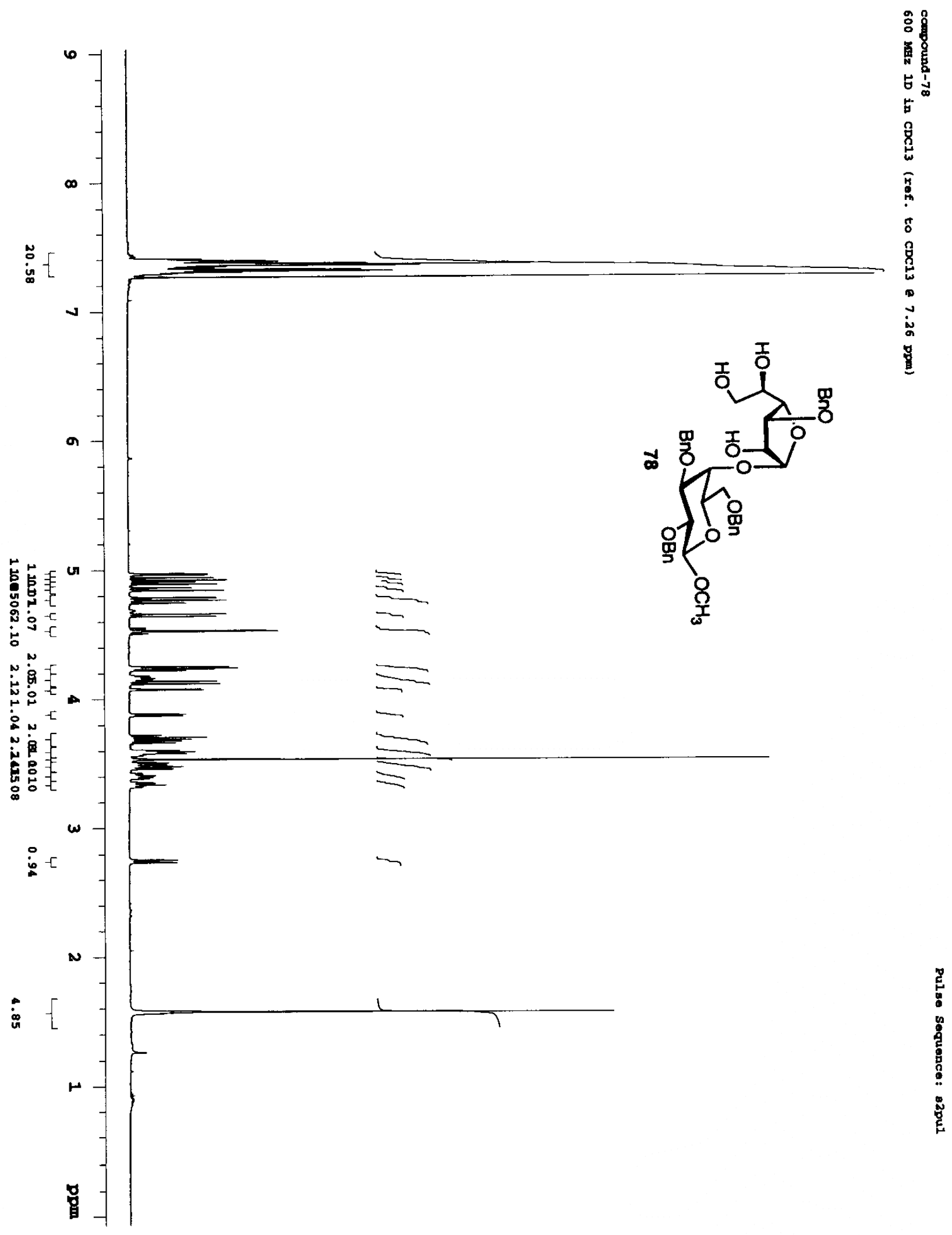




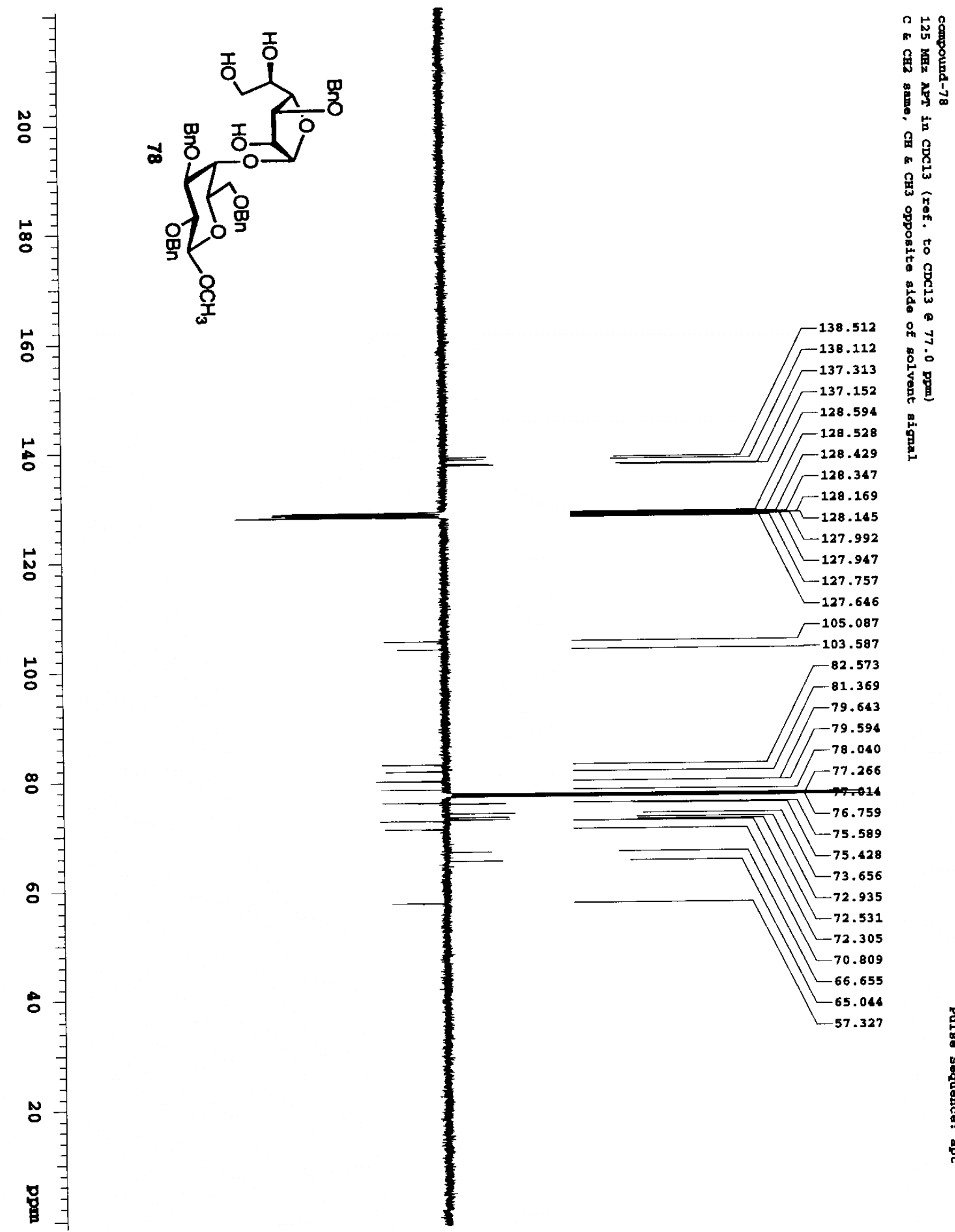



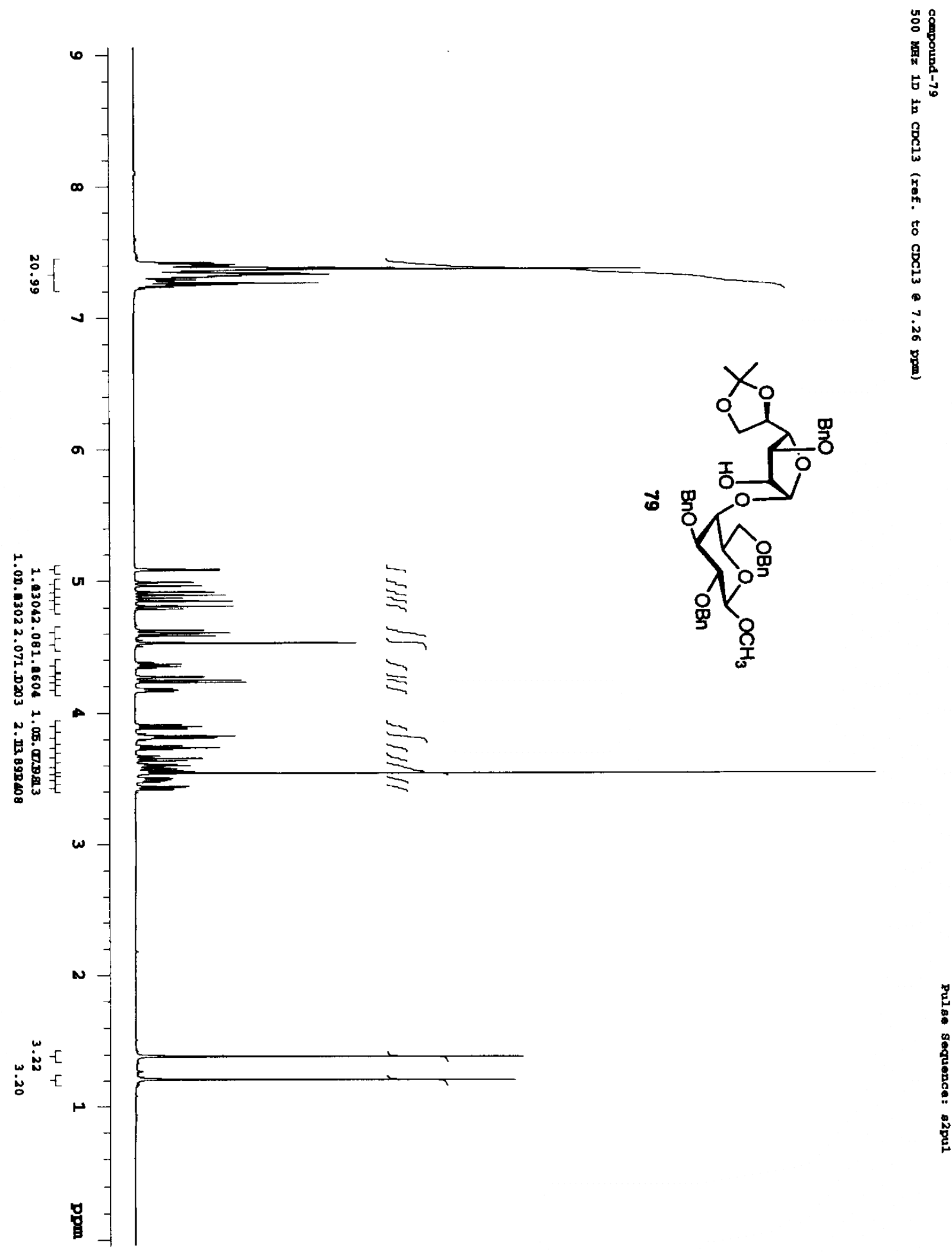


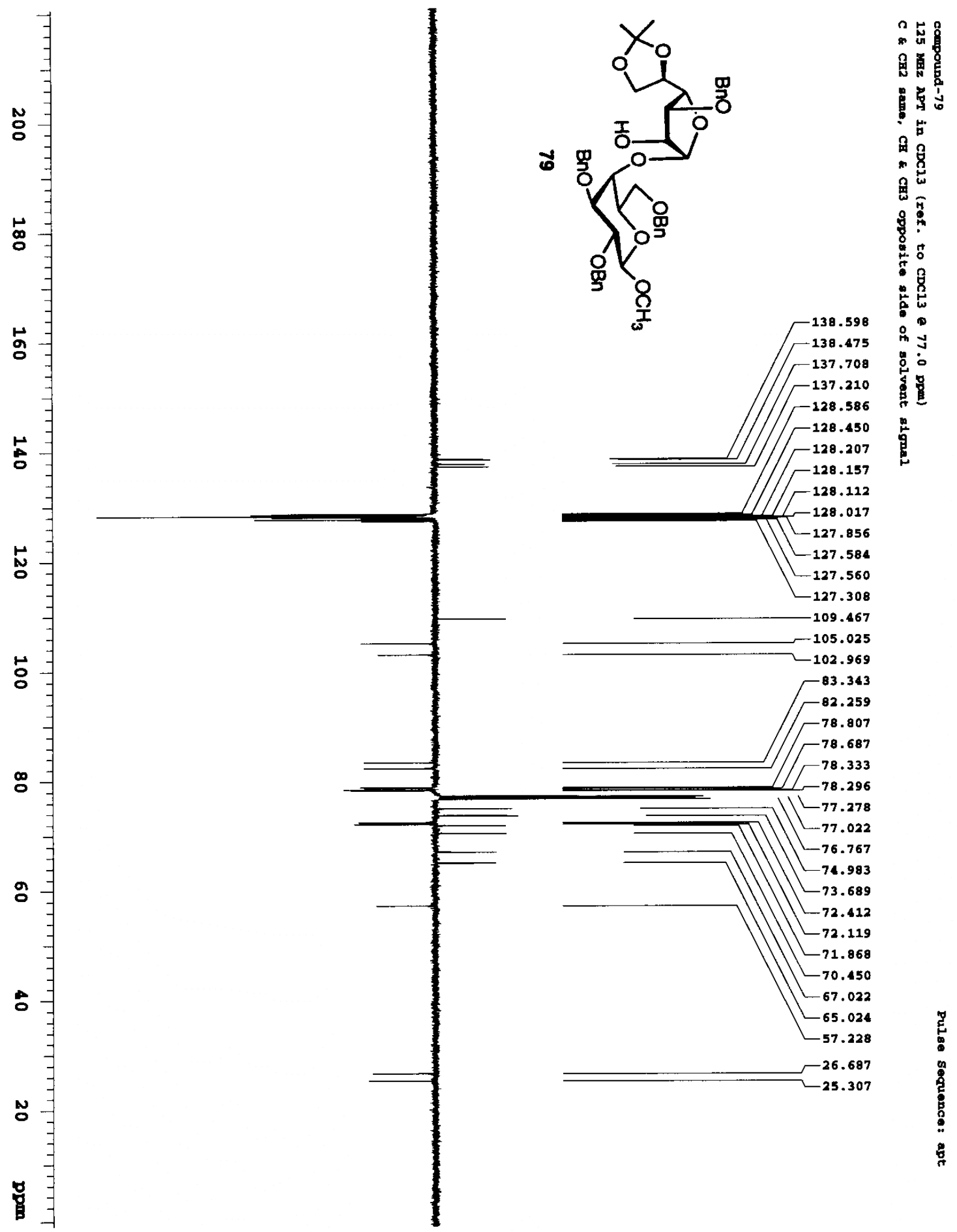




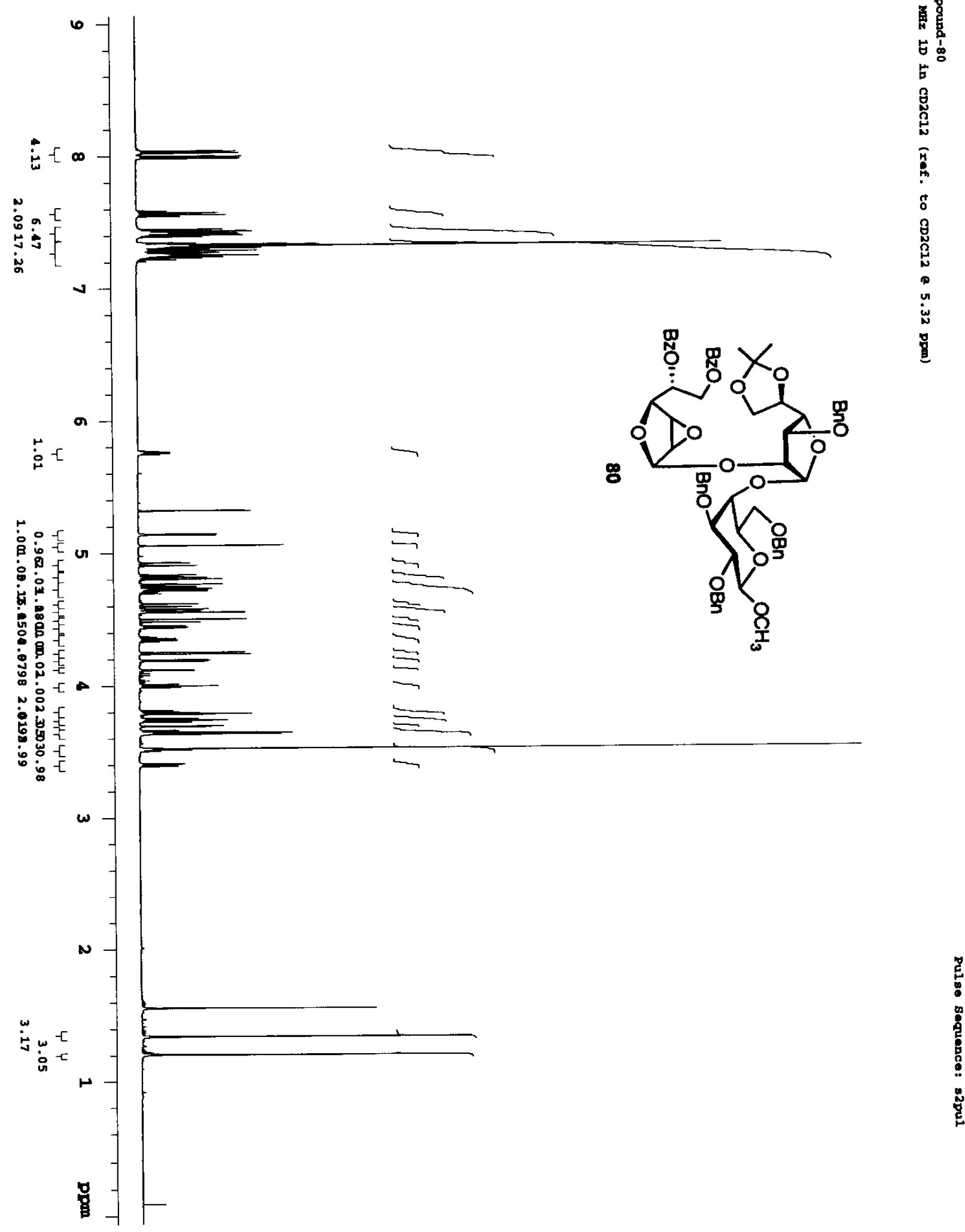




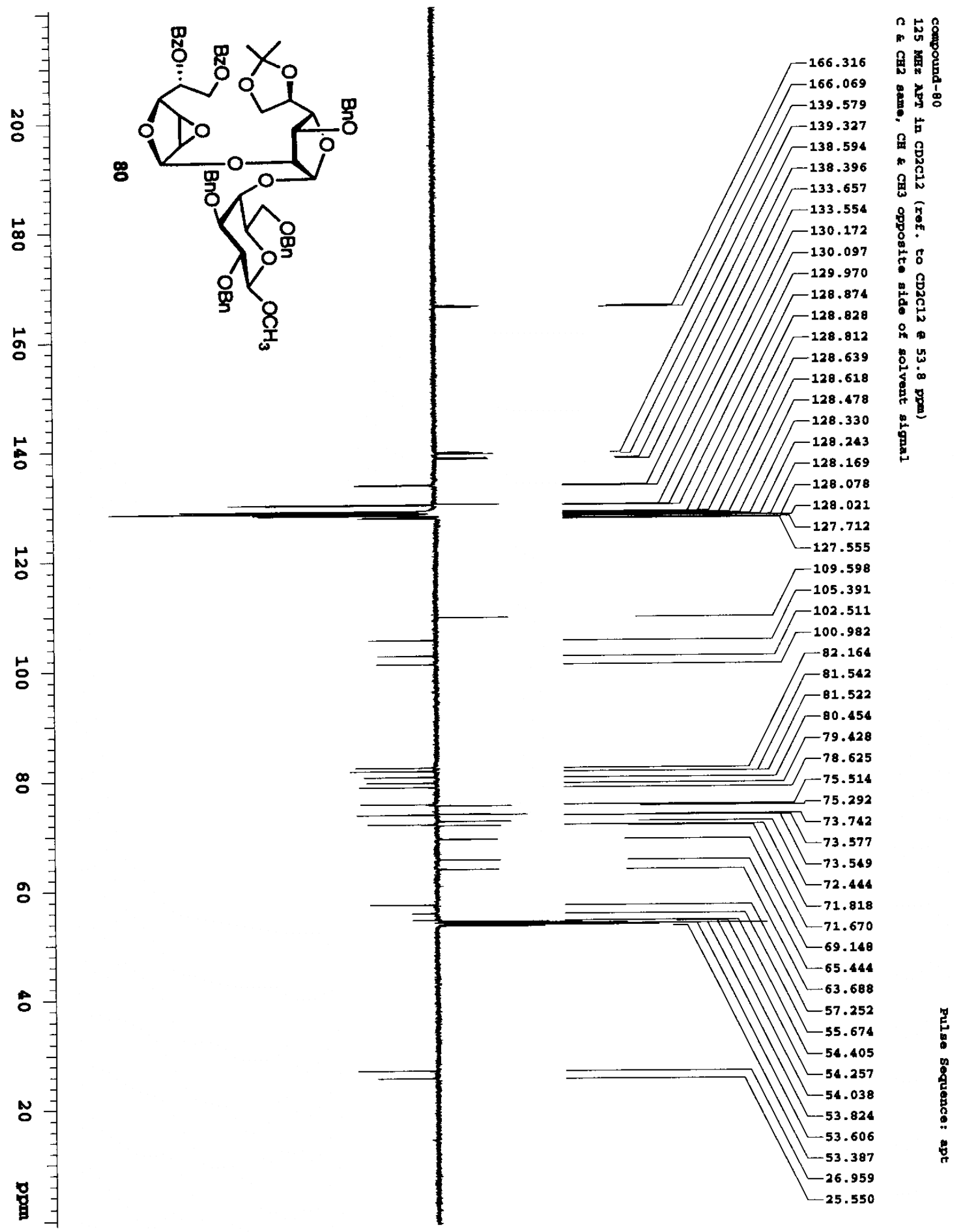




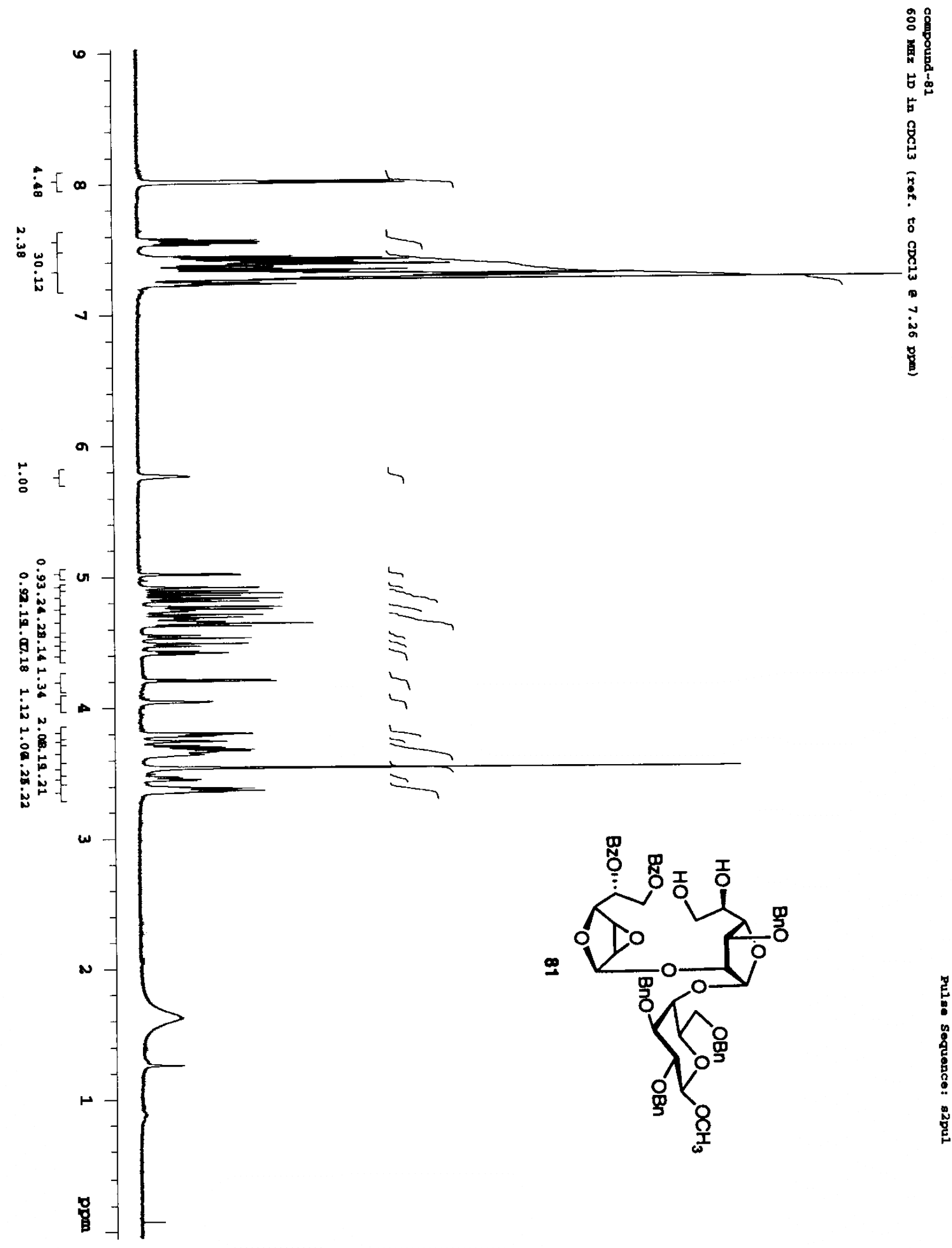




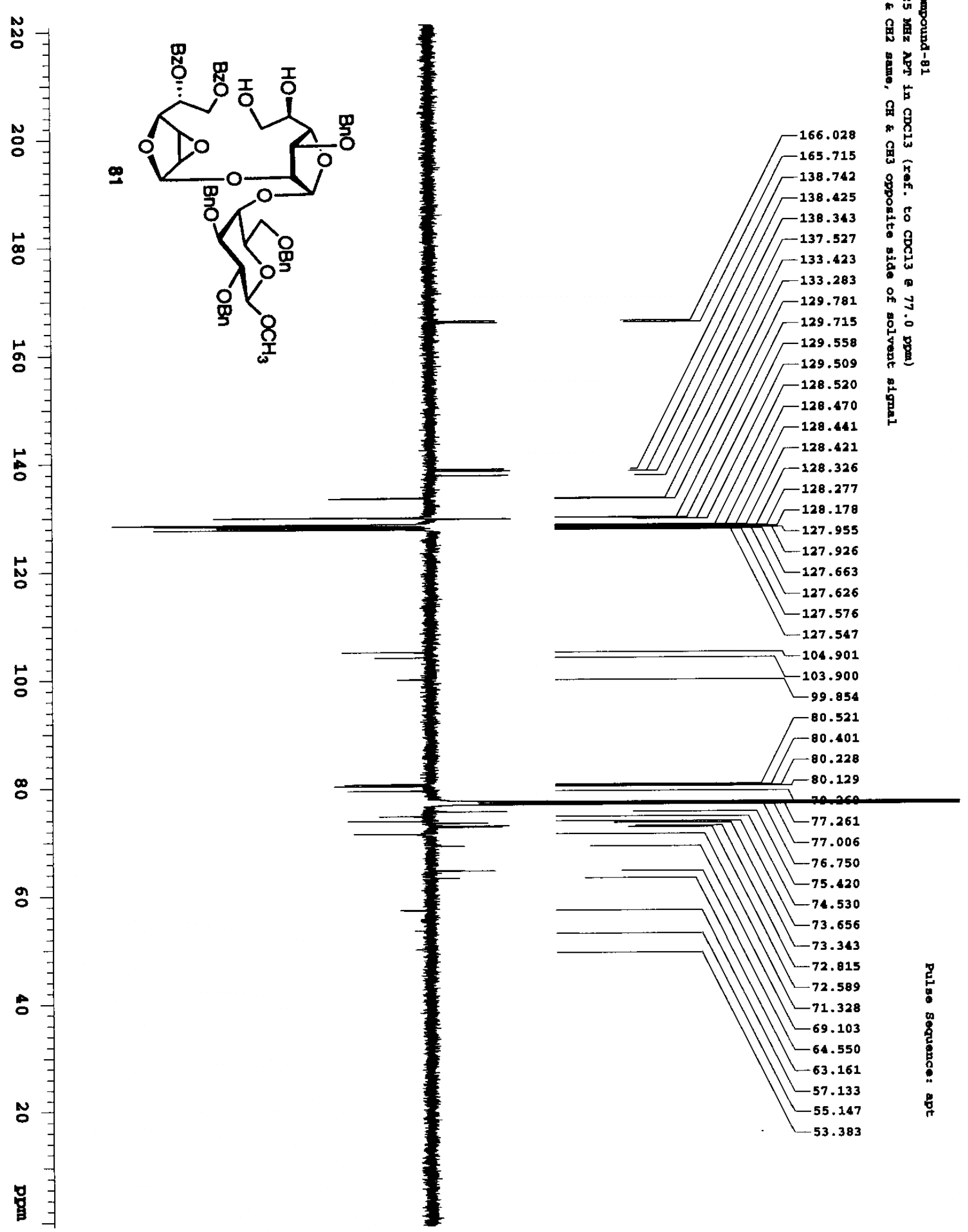




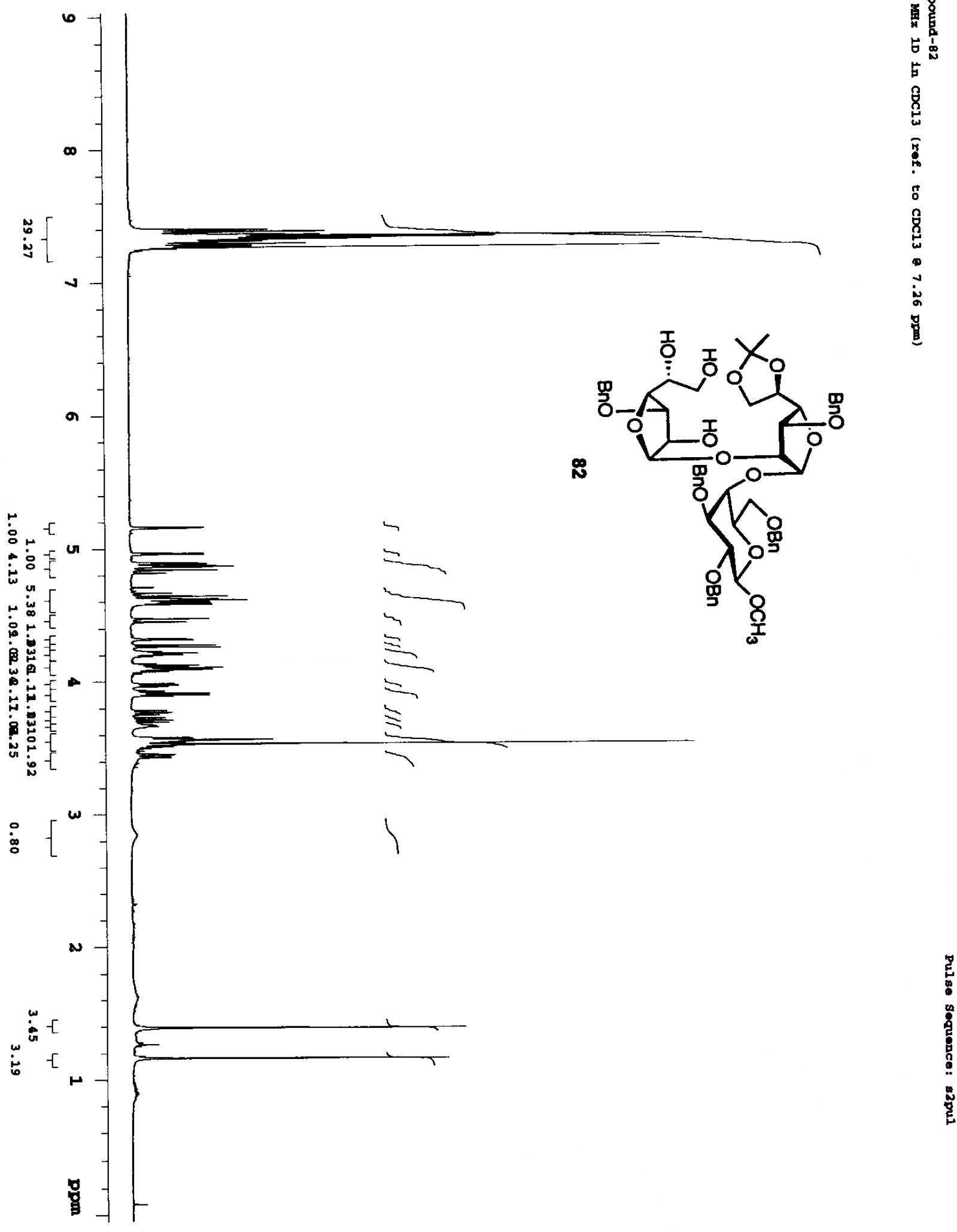




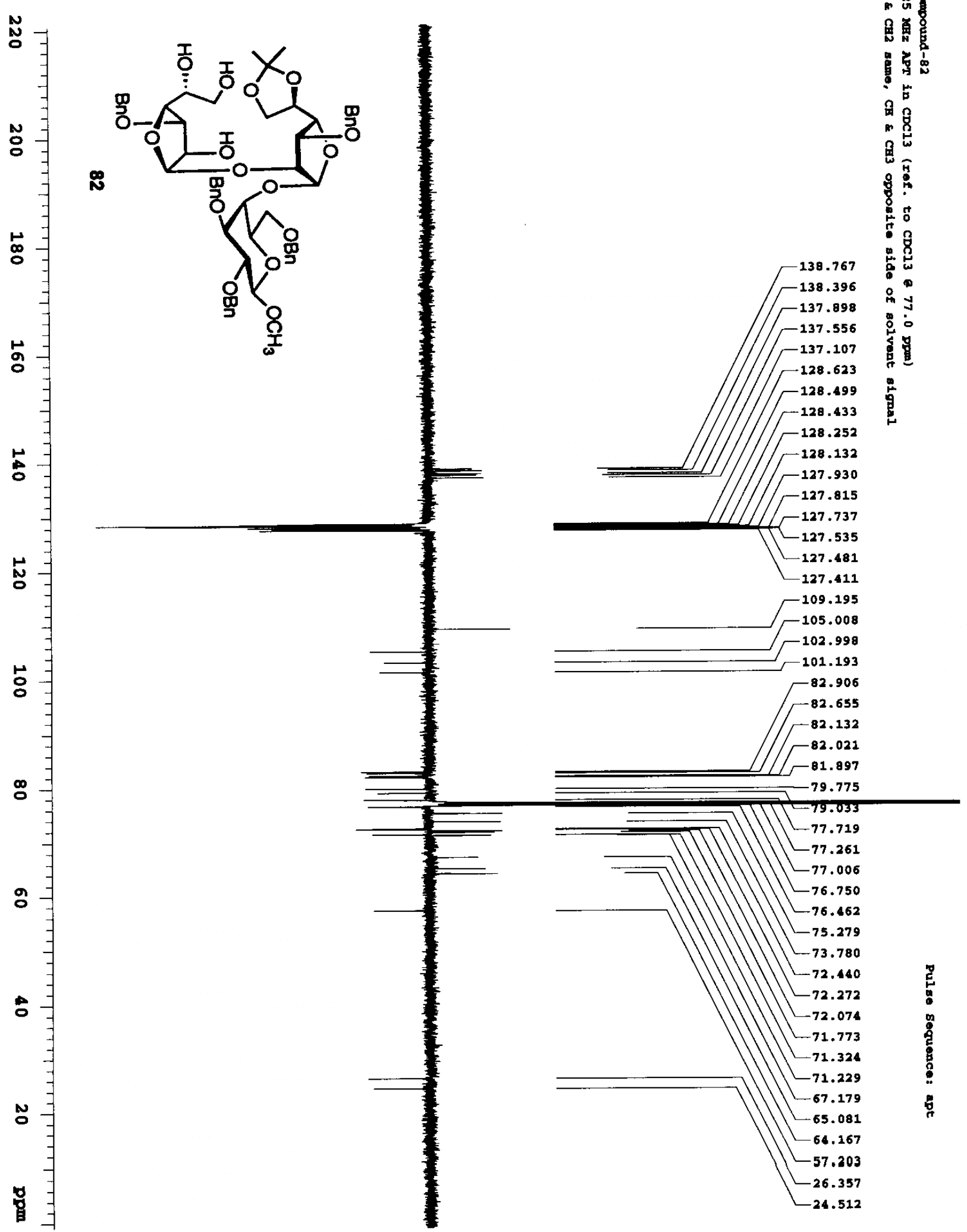




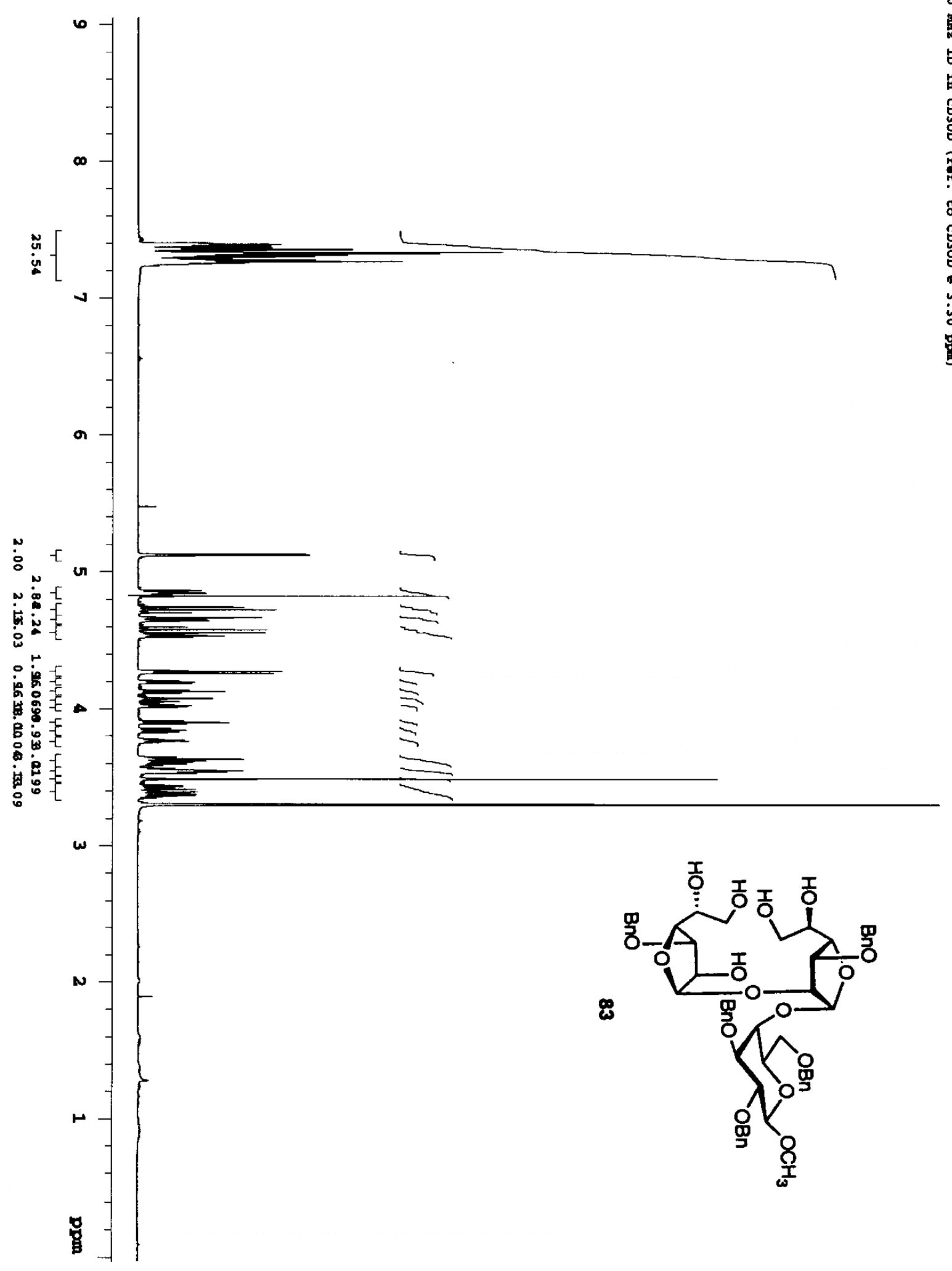

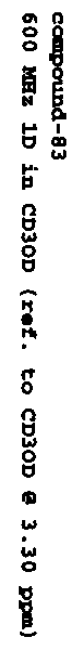



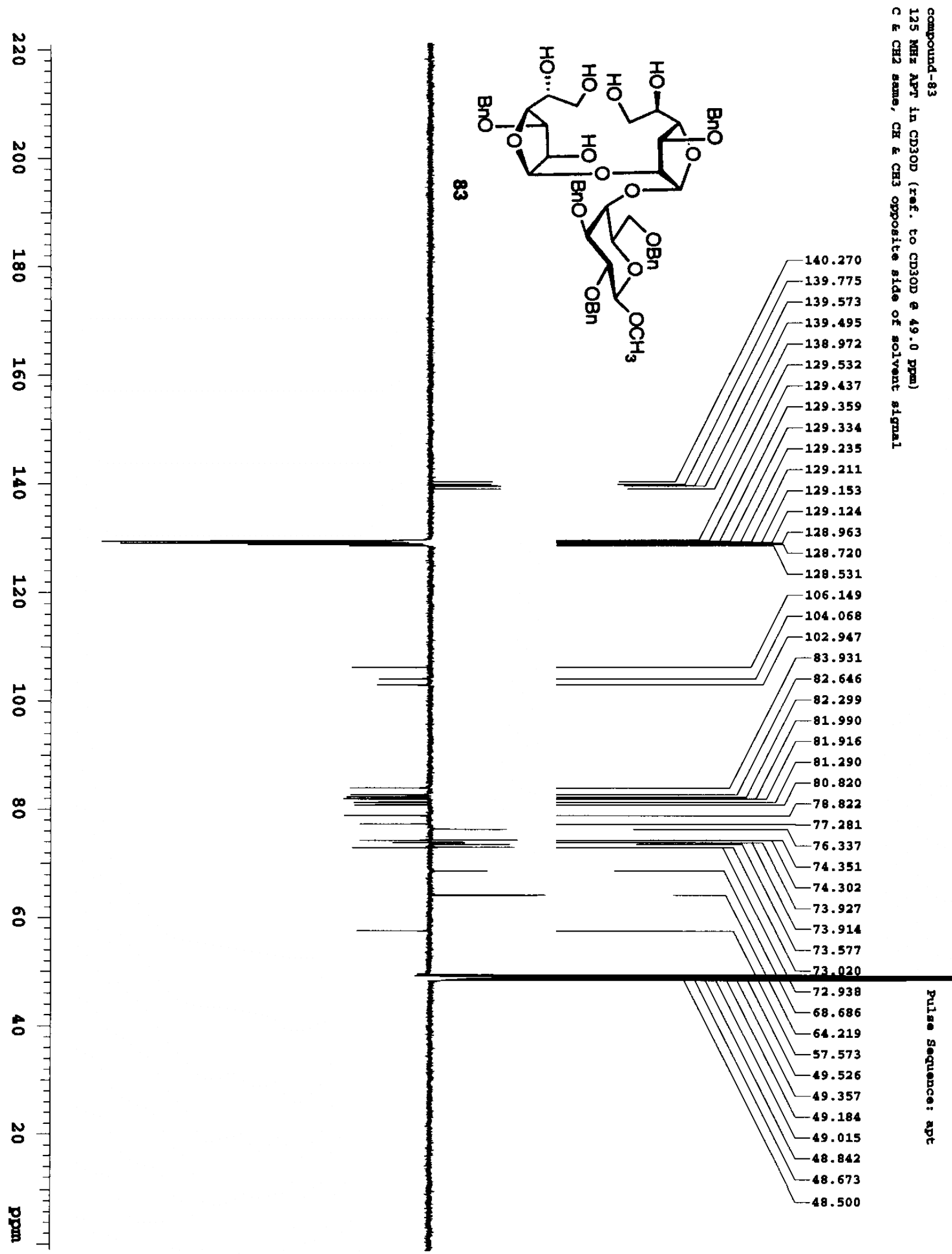

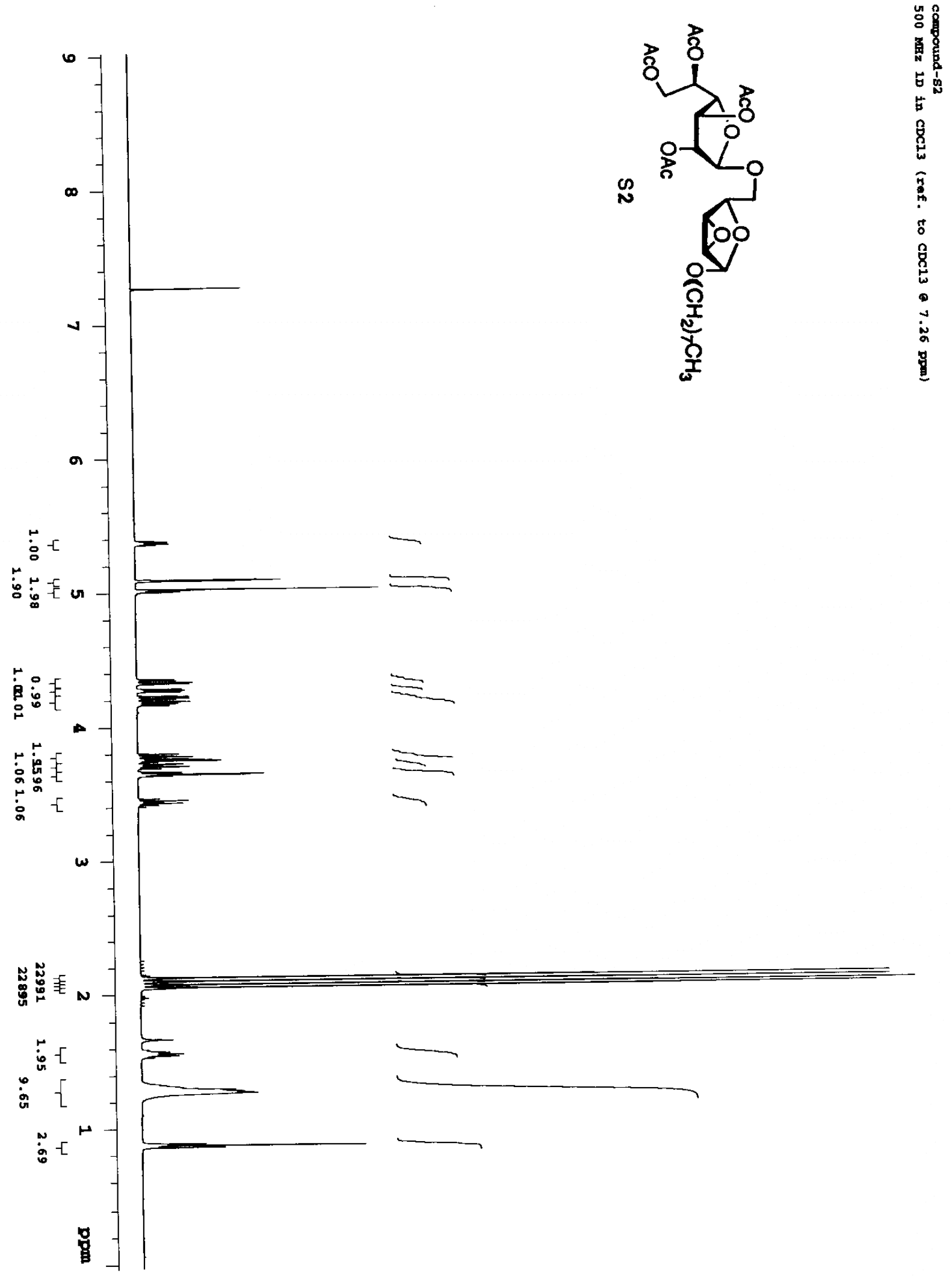


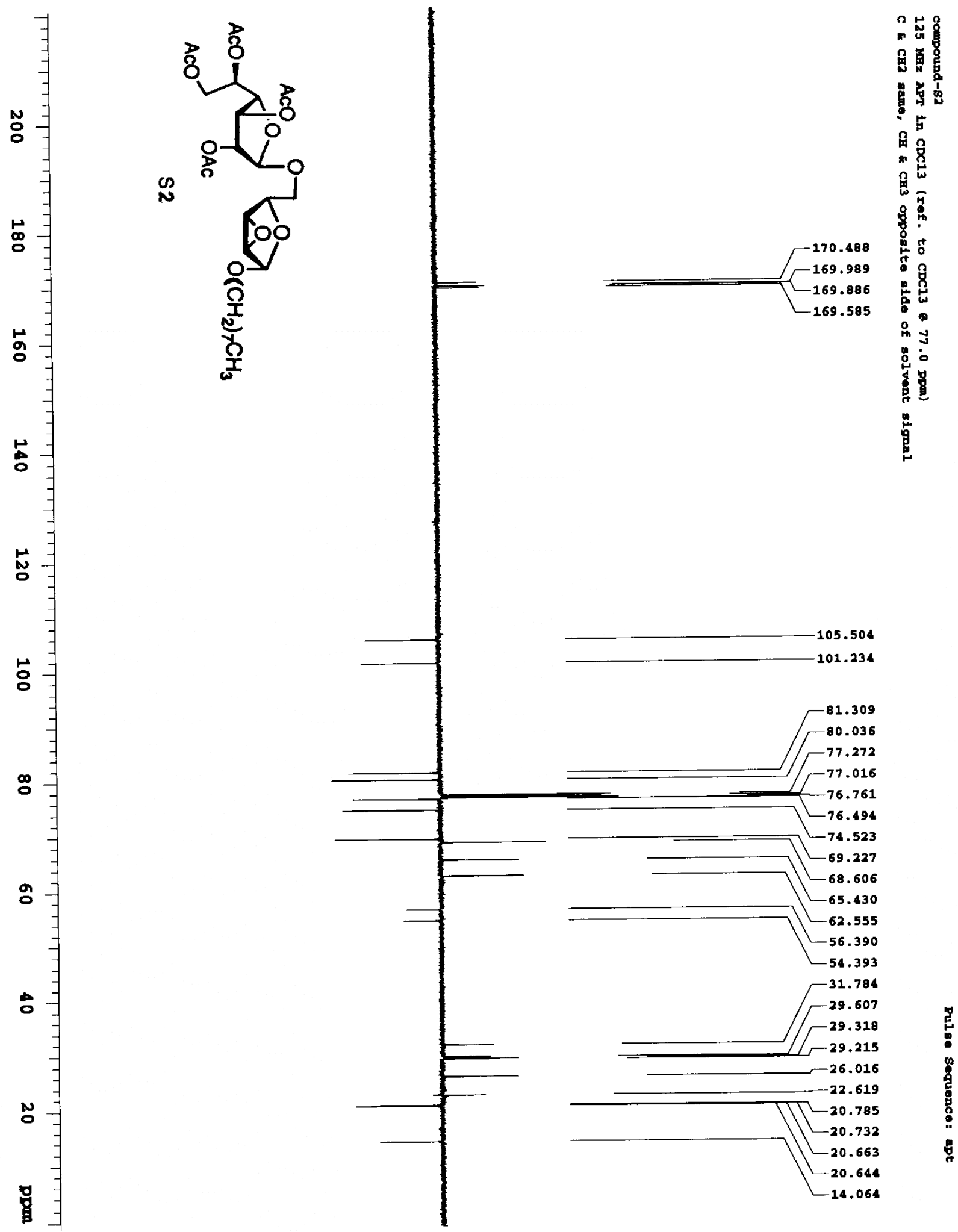



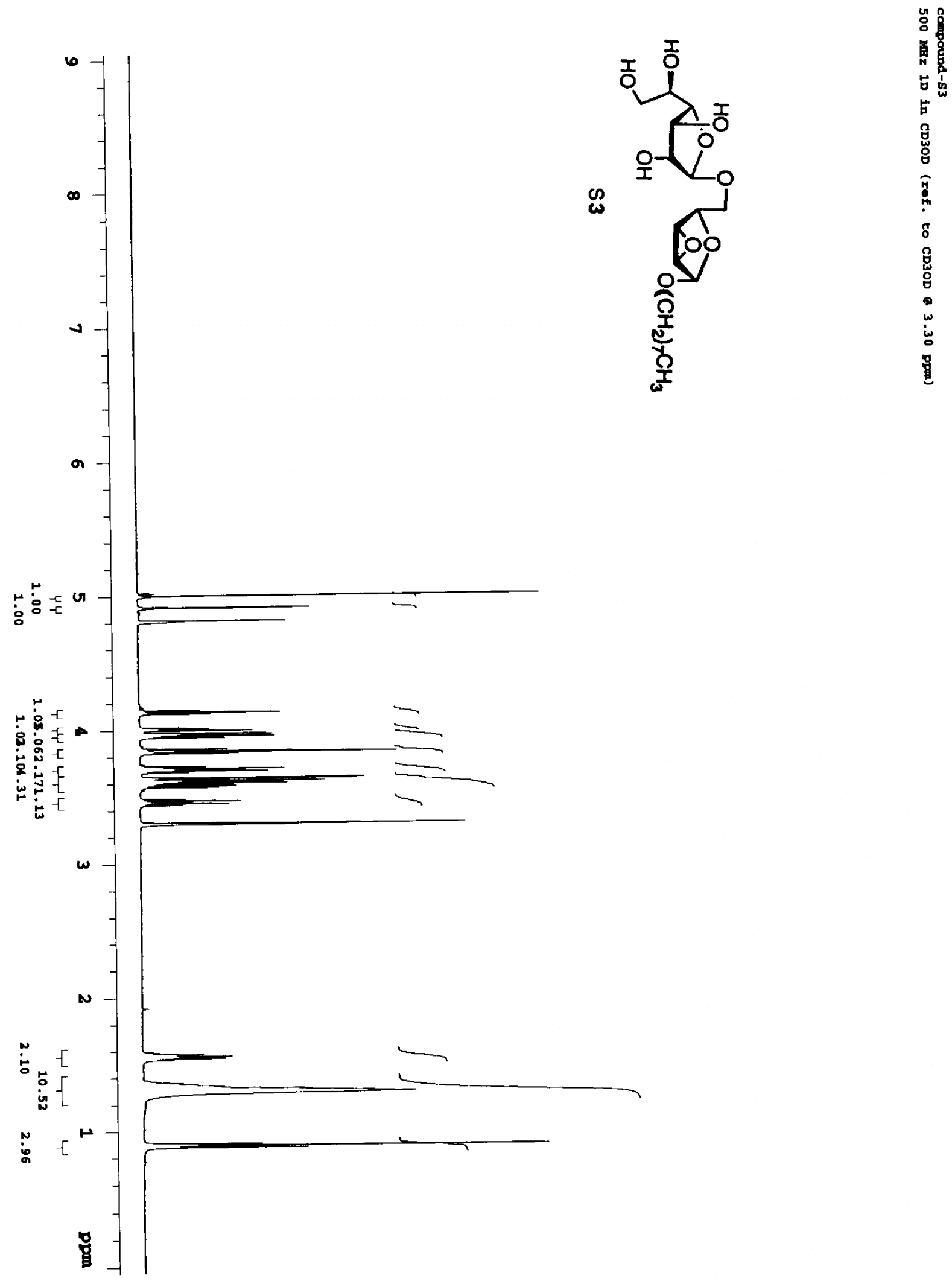

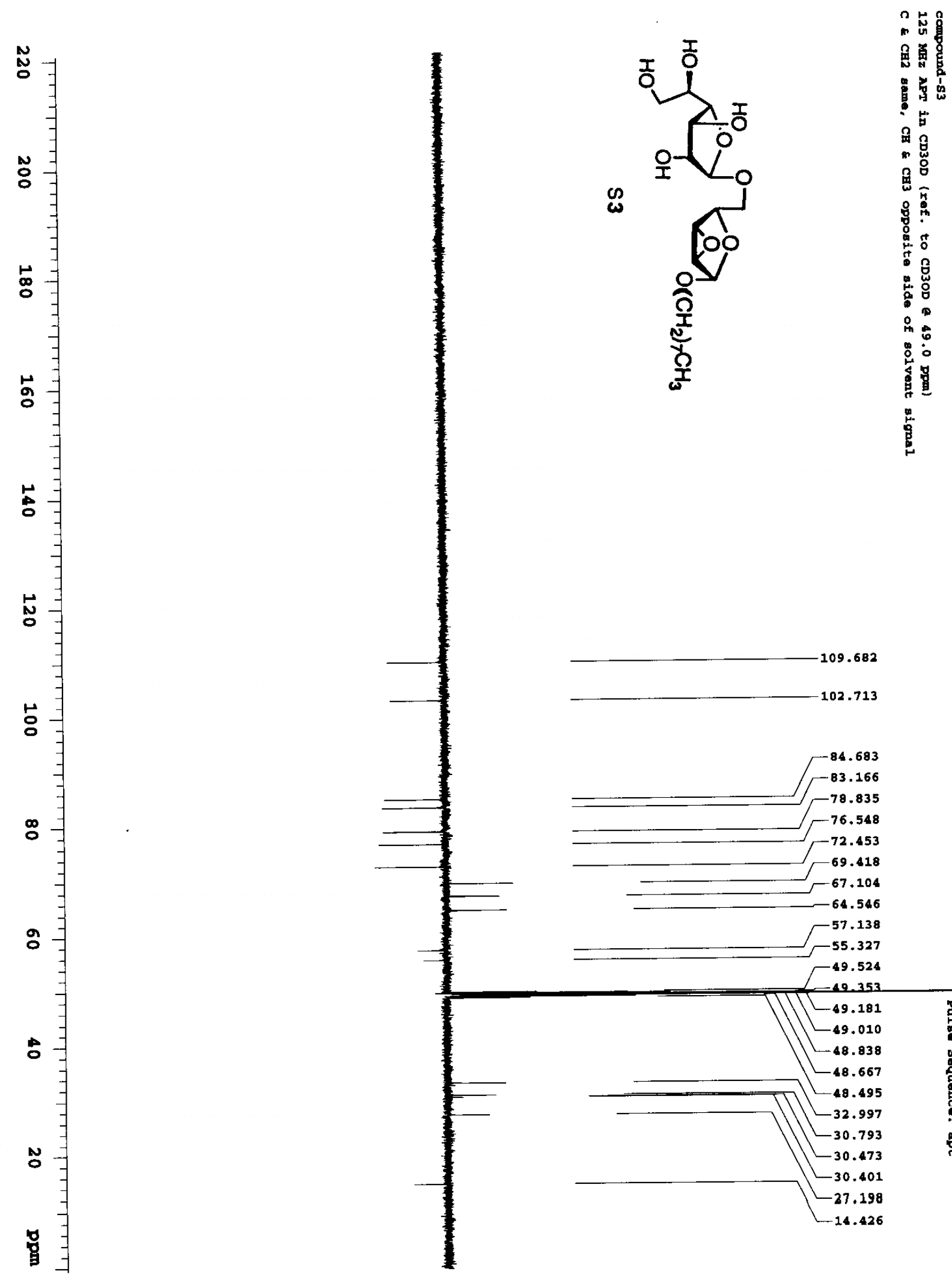


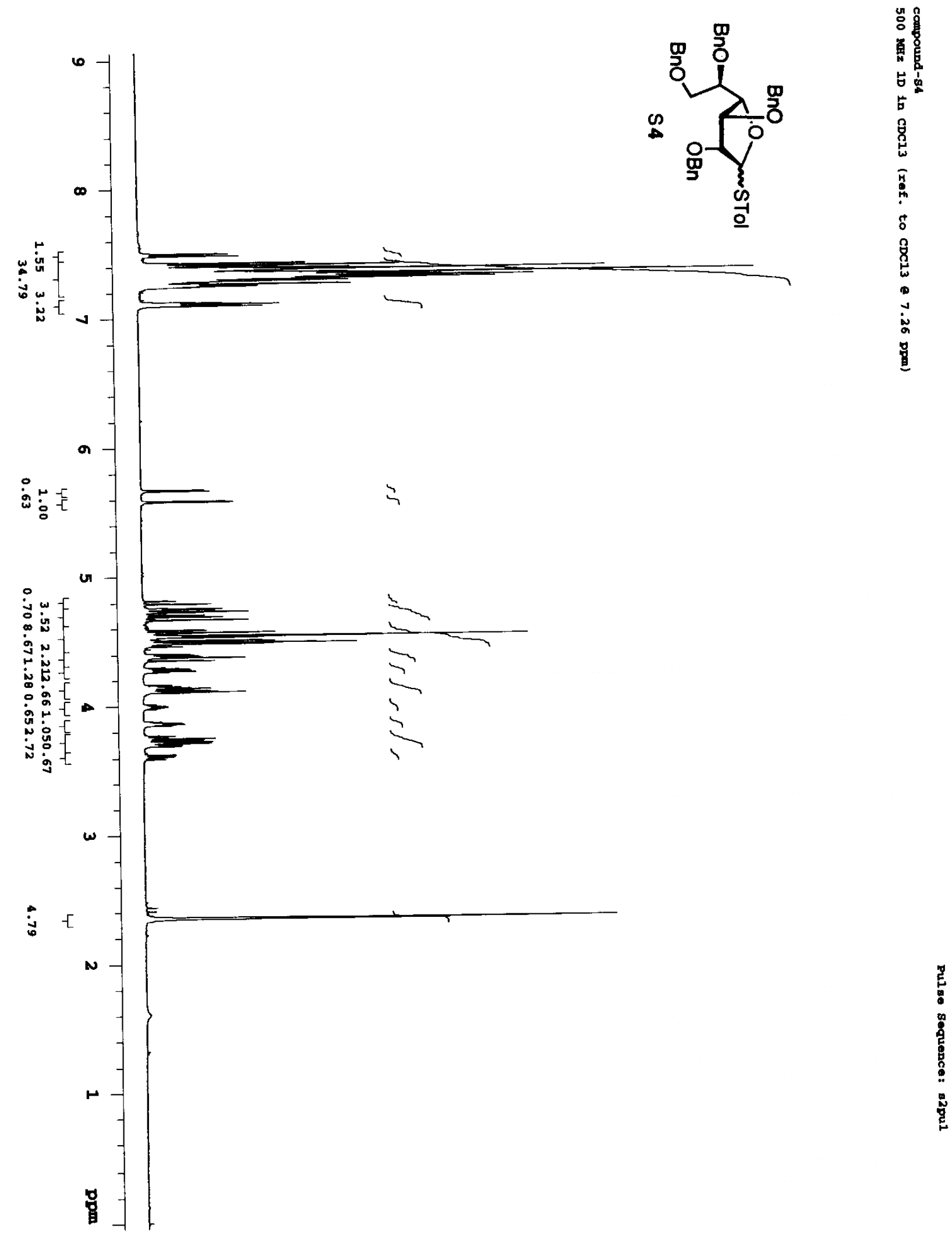




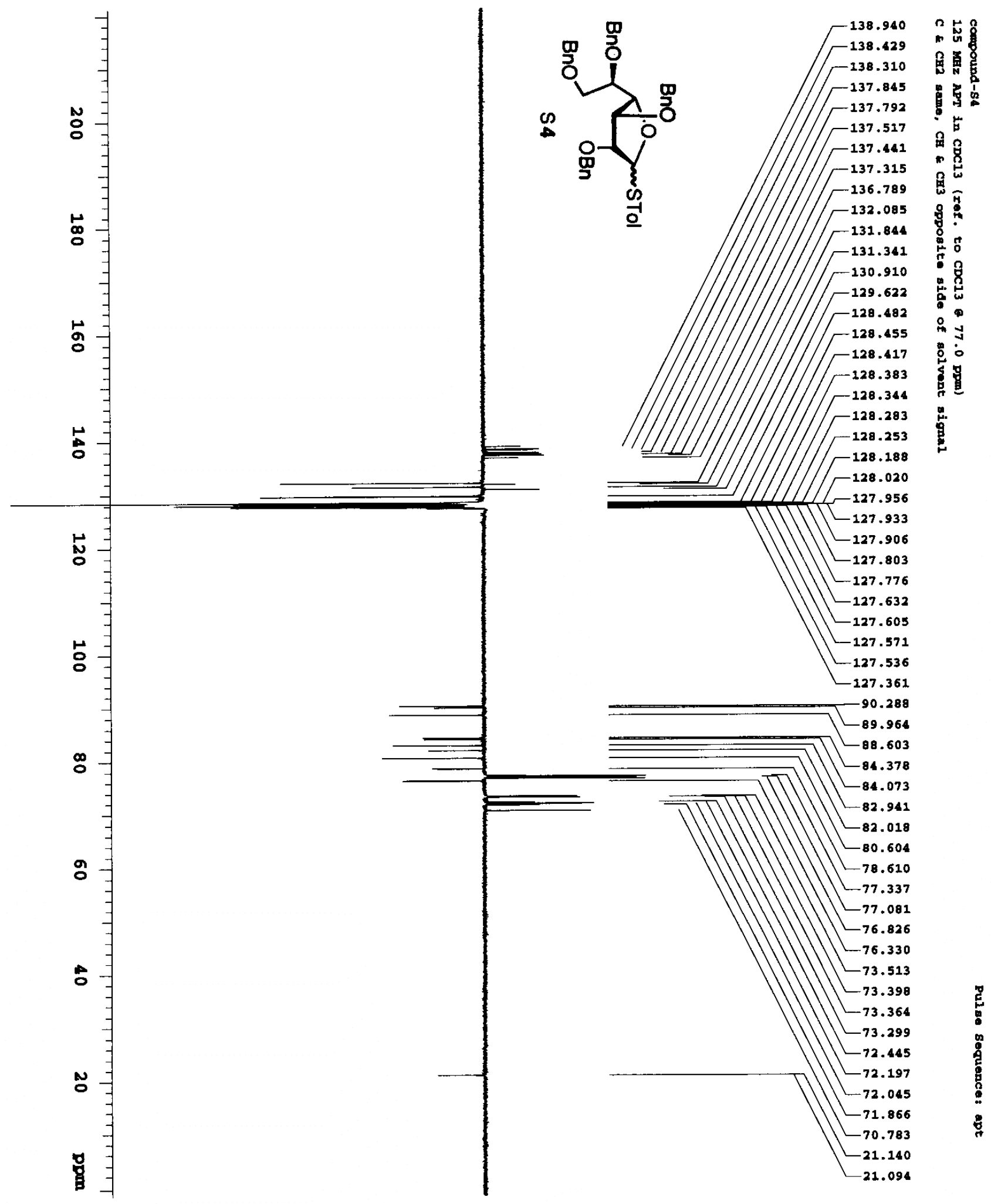



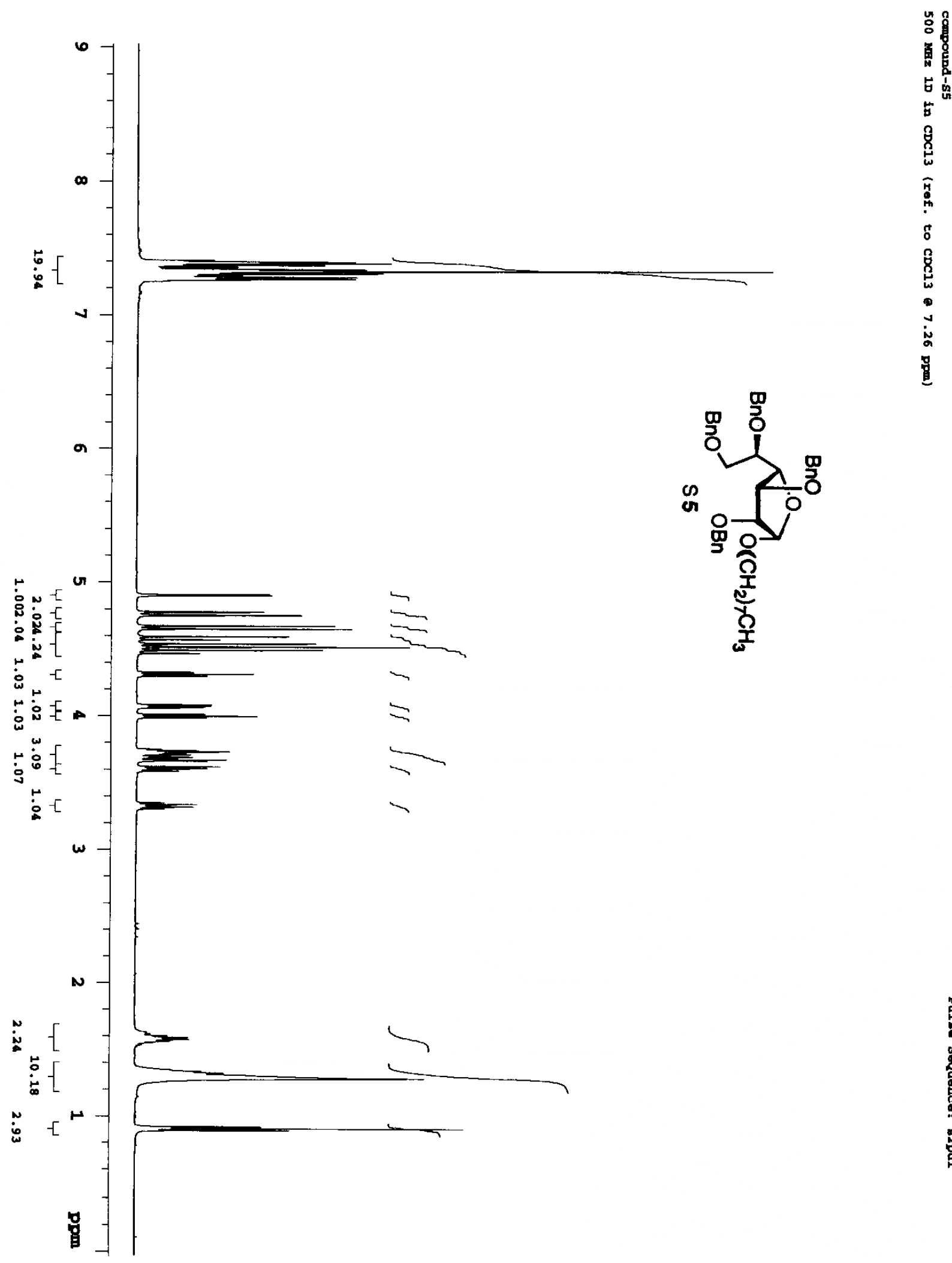


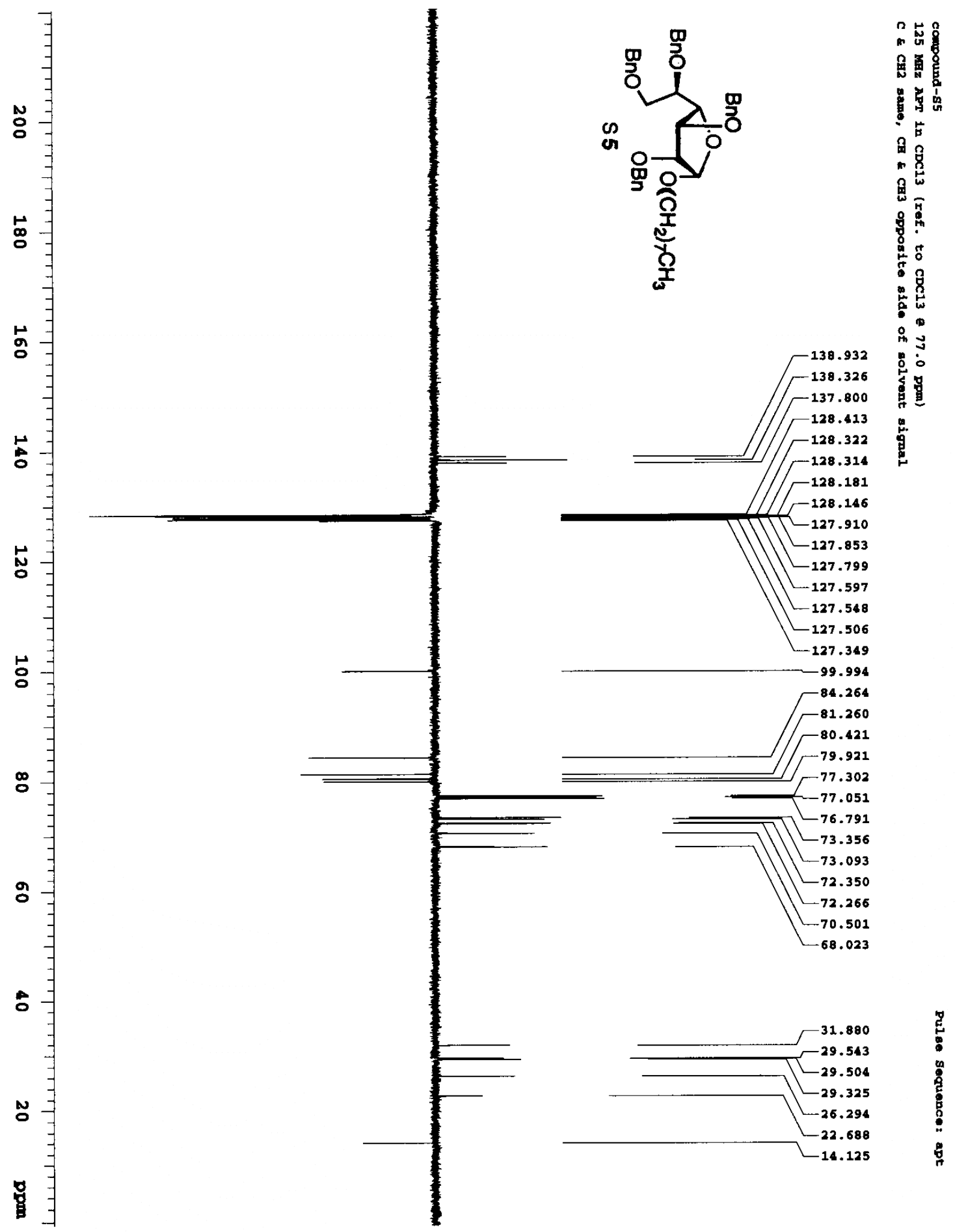



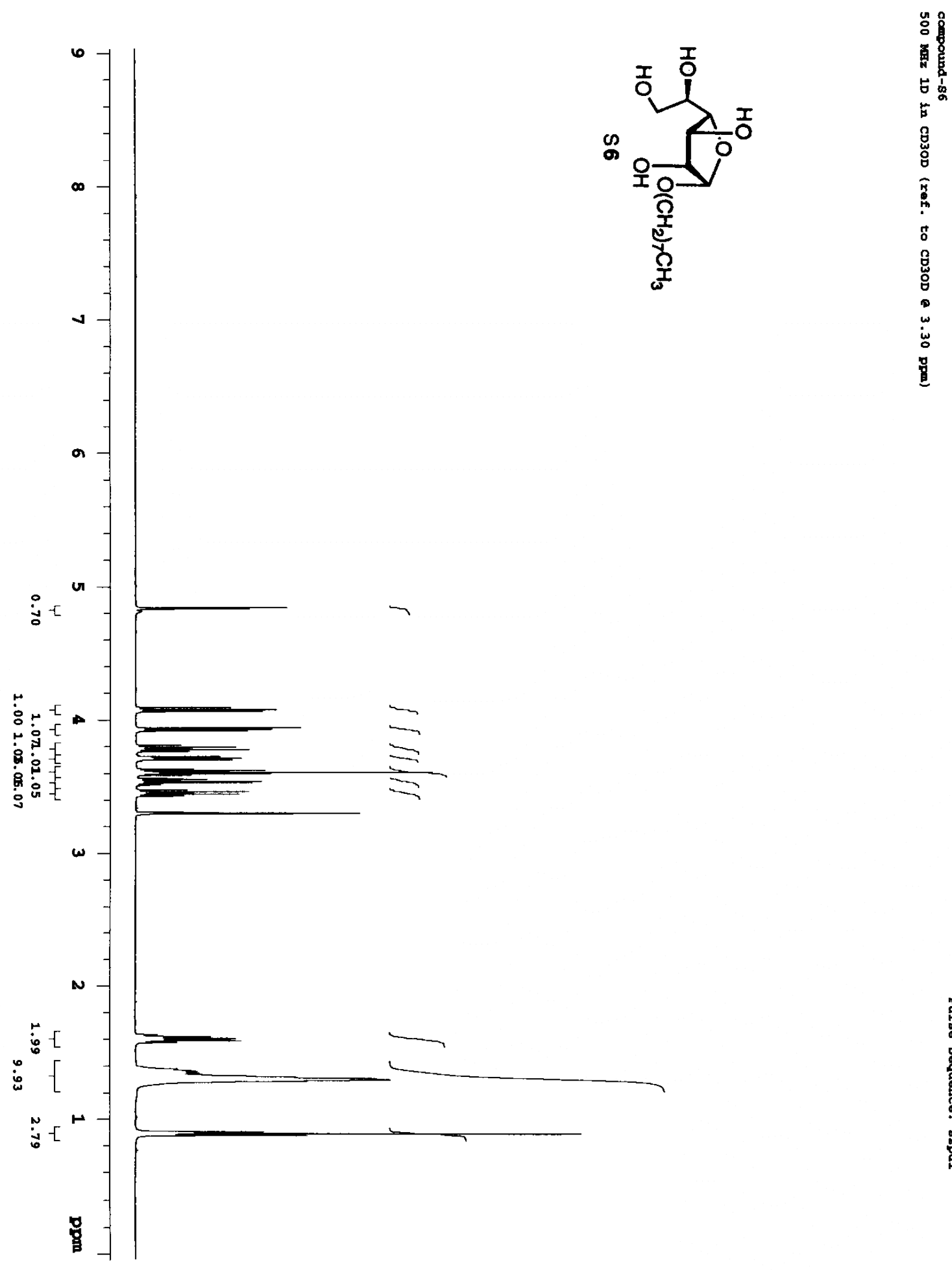


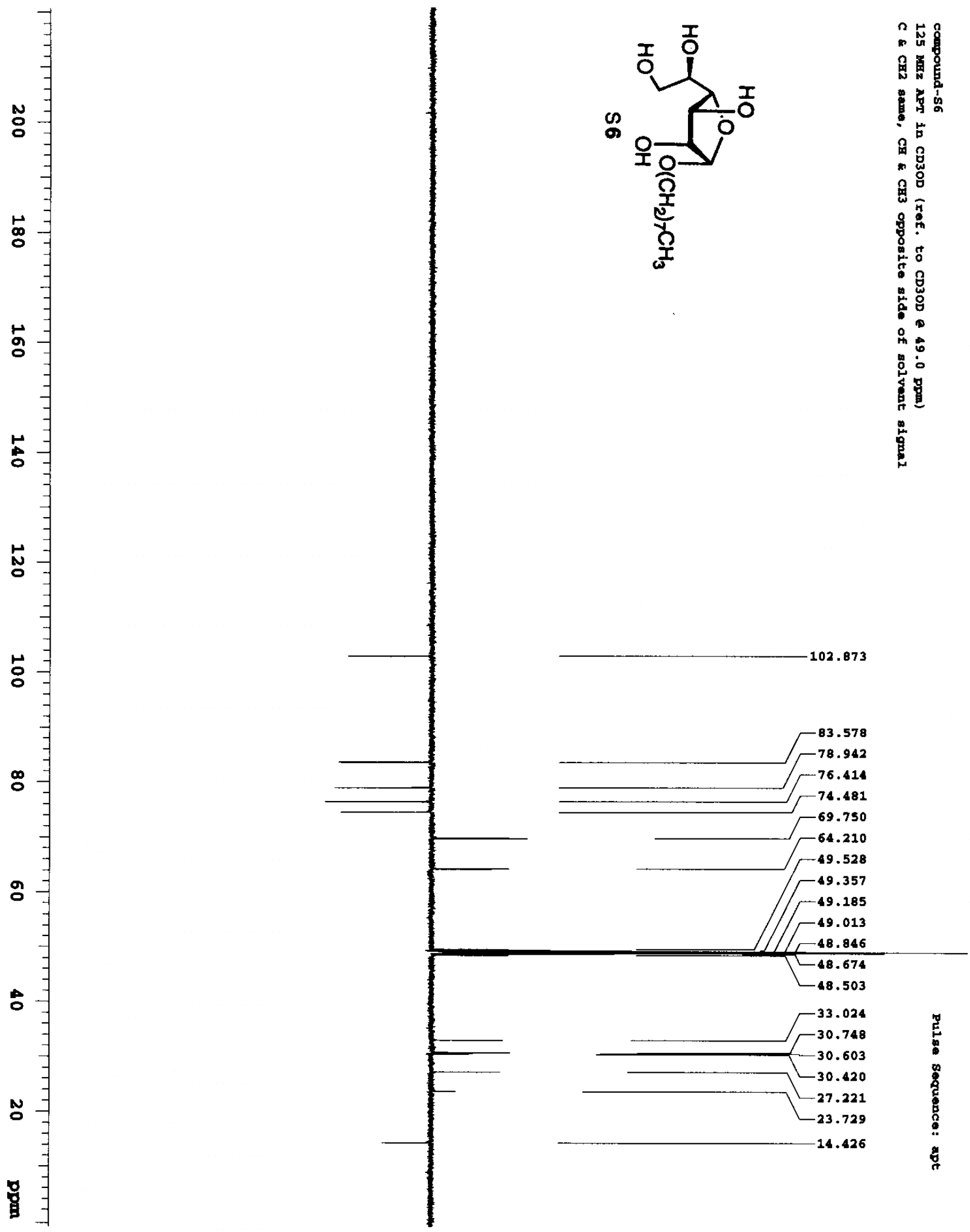




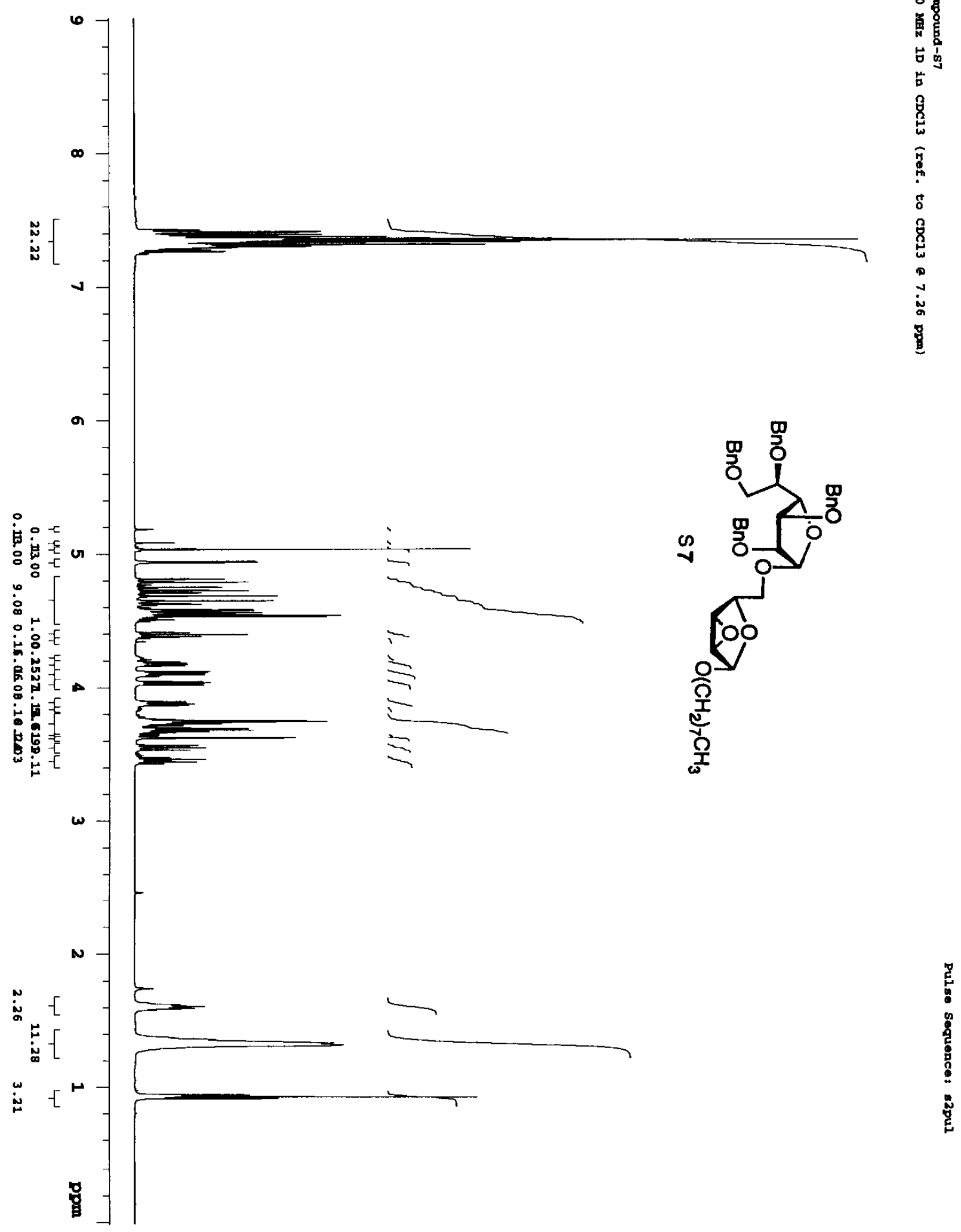




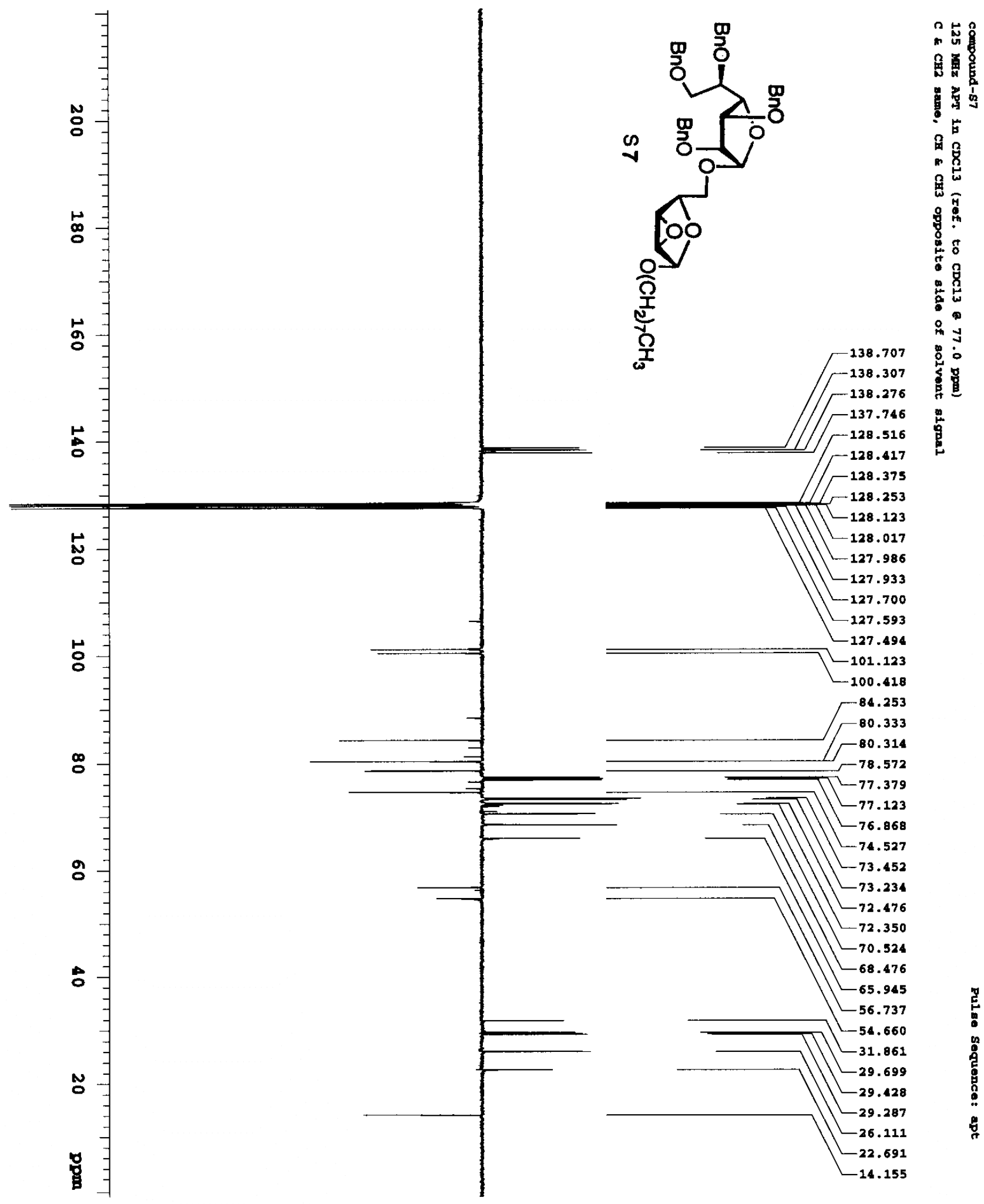




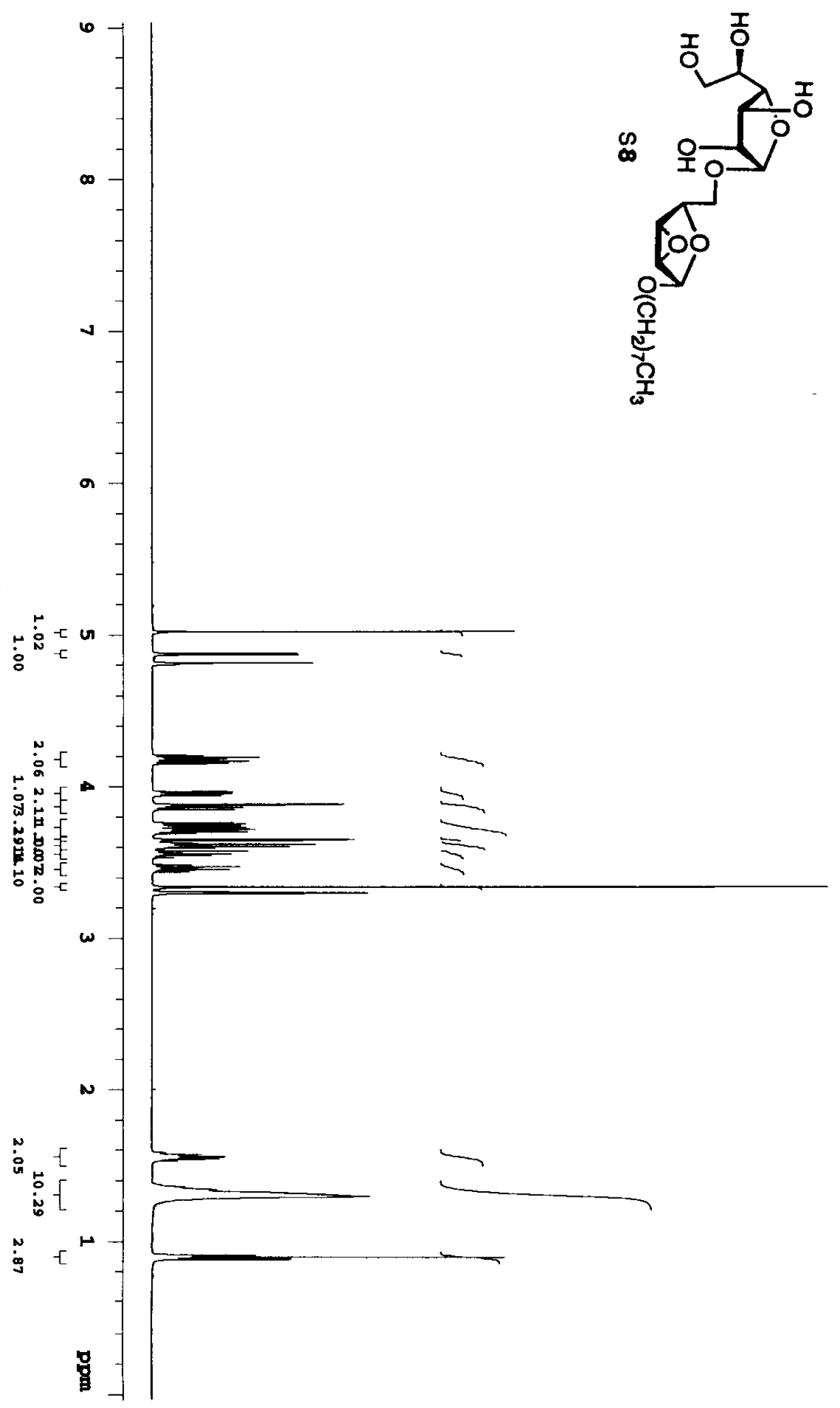

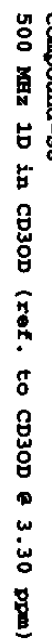



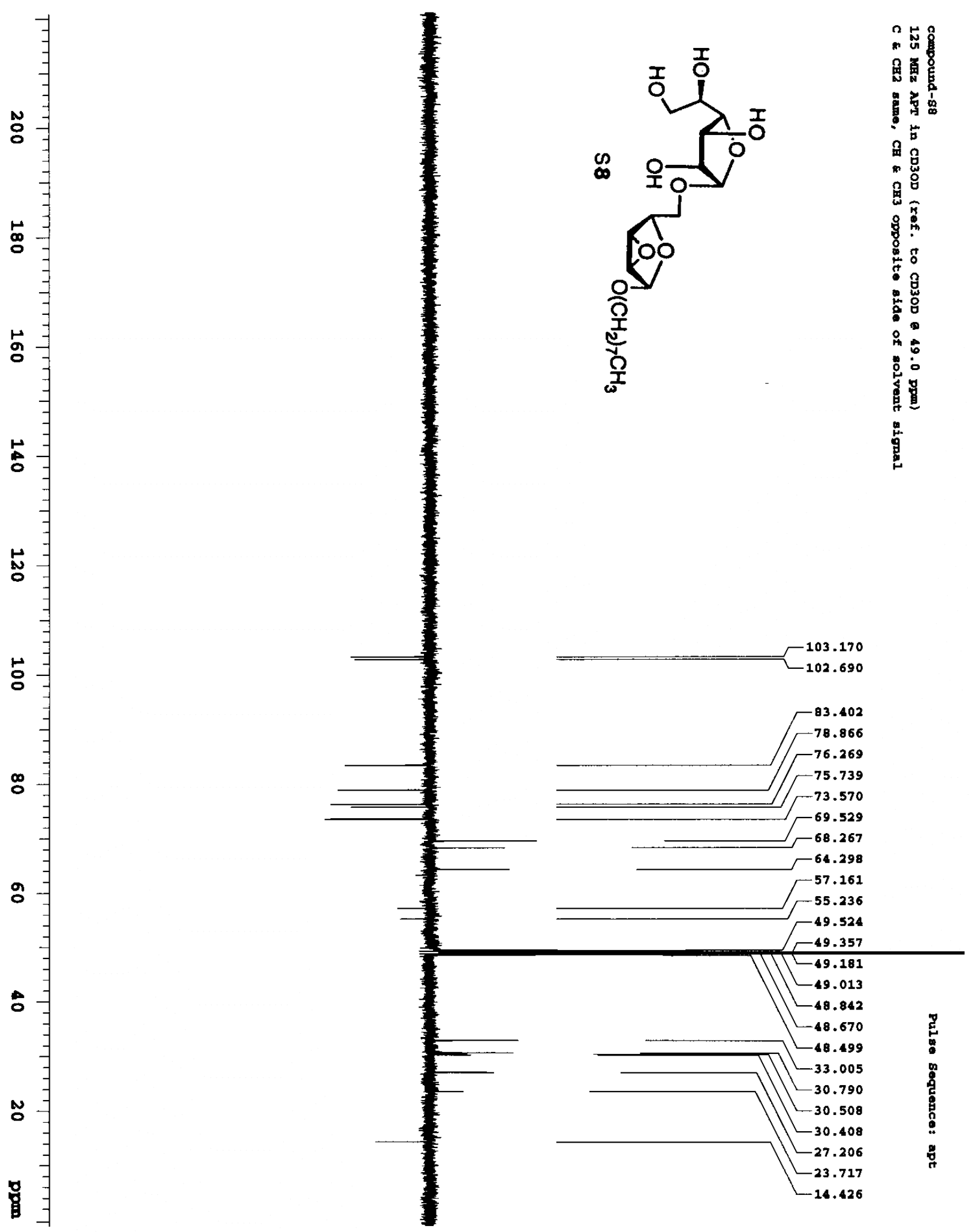